\title{
THE EFFECTS OF MICROTUBULE
}

\section{STABILIZING DRUGS ON MACROPHAGE IMMUNE-MEDIATED ENDOCYTOSIS.}

\author{
By
}

Praneta Joshi

A thesis submitted to Victoria University of Wellington in fulfilment of the requirements for the degree of Master of Biomedical Science.

School of Biological Sciences

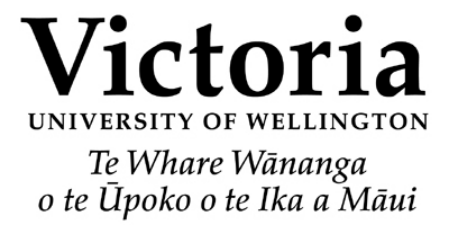

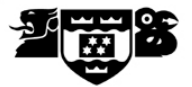

2010 
ABSTRACT

Microtubule stabilizing drugs (MSD) bind and stabilize microtubules, thus inhibiting their normal function. MSD exhibit anti-mitotic effects which makes them attractive as cancer chemotherapeutics and much of existing research has focused on these effects in proliferating cells. In contrast, we are interested in assessing the effects of microtubule stabilization on non-proliferating cells, such as macrophages, to determine potential mitosis-independent actions of MSD on microtubule function. Thus, we investigated the effects of MSD on macrophage receptor-mediated endocytosis of low density lipoproteins (LDL) and found no significant effect on the ability of paclitaxel-treated macrophages to endocytose LDL.

Alterations to macrophage phagocytic and killing efficiency due to treatment with paclitaxel, peloruside or docetaxel, as well as the recently discovered compounds, ixabepilone, mycothiazole, and zampanolide were investigated. Treatment with paclitaxel, peloruside or docetaxel did not significantly inhibit phagocytosis or killing of bacteria. Results from confocal microscopy suggest that paclitaxel alters phagocytic kinetics in macrophages. Respectively, zampanolide and mycothiazole significantly inhibited macrophage bactericidal and killing ability, while Ixabepilone enhanced bacterial killing. MSD treatment also altered production of tumor necrosis factor alpha (TNF- $\alpha$ ) and nitric oxide (NO) during bacterial killing. Optimal activation of macrophages with IFN- $\gamma$ did not alter the effects of MSD. Taken together, these results suggest that MSD have multiple immunomodulatory effects unrelated to their anti-mitotic effects. The data suggests that during MSD treatment, macrophage activity maybe altered or impaired, thus modifying the ability of patients to fight off bacterial infections. 


\section{ACKNOWLEDGEMENTS}

First and foremost I would like to sincerely thank my supervisor Dr Anne La Flamme, who has supported me throughout my thesis with her patience, encouragement and extensive knowledge, while still allowing me the freedom to work in my own way. The level of my Masters thesis would not have been achieved without her excellent guidance and encouragement, and for this I am truly grateful to her.

I would also like to thank Dr Bronwyn Kivell and Sushila Pillai for their support and guidance with the confocal microscope. To Bronwyn, thank you for the valuable assistance, and also the extra time on the machine. To Sushila, I could never have done it without you. Thank you for all your guidance, and support, especially when the long experiments meant staying back late.

I would like to thank Laura Green, for all her advice (and countless discussions) on RAW cells and tissue culture. Many thanks also to Marcus Robinson for his ideas and guidance from the early stages of this project, especially regarding LPS, macrophages and MSDs. Thank you for sharing your expertise and ideas. Additionally, I would like to thank Rekha for all your help with J774s and lending me the Image-it dye. I am grateful we could discuss phagocytic assays (and why they don't always work.)

Ariane and Dave, you are both amazingly knowledgeable and I am so grateful to both of you for always being there to discuss any lab work I was doing. Ariane, thank you for the innumerable discussions on microtubule dynamics and MTT assays. Dave, thanks for being there to discuss anything and everything. Bridget, I would like to say a big thank you for listening to me whinge when things didn't work out, for helping me with the confocal, and for always having a song for us in tissue culture! Miles you are a computer expert extraordinaire! I will forever be grateful for all your help with Word, Prism and Endnote. Also thank you for always being ready to listen and help no matter what stage of writing you were at, and for providing help via email when you were in OZ!

To everyone else in KK601, all the LAFs, JHMs and BKs thanks for all the support and making the lab a happy place to work (and live) in. A special thank you for Jenni for being so lovely and always being there for me. I would also like to acknowledge Jonnel for being such a smiley and great person to work with right from $1^{\text {st }}$ year.

Finally, I am thankful for my wonderful parents, who have always supported me and given me all the opportunities I could ever have wanted to follow my dreams. I would like to express my love and gratitude to mum, dad and Bill for your understanding and support throughtout my years of study. Special thanks to my mum - there is nothing I can say that will express all that I feel, so thank you. I am also thankful to my little brother for always fixing my laptop when I manage to ruin it.

This thesis is dedicated to my parents, Pankaj and Priyanka Joshi. 
TABLE OF CONTENTS

$\begin{array}{ll}\text { Abstract } & \text { ii }\end{array}$

Acknowledgements

Table of contents $\quad$ iv

List of tables and figures vi vi

Abbreviations $\quad \mathrm{X}$

Chapter 1: Introduction 1

1.1 Microtubules and Microtubule targeting agents 1

1.2 Macrophage Functions and Immune Responsiveness 13

1.3 Summary of Known Immunological Effects $\quad 27$

1.4 Research Aims and Hypothesis $\quad 27$

Chapter 2: Materials and Methods $\quad 29$

2.1 Reagents $\quad 29$

2.2 Cell Culture 29

2.3 Receptor-mediated endocytosis 31

2.4 Phagocytosis assay - GFP-labelled E.coli phagocytosis 32

2.5 Phagocytosis assay - pHrodo phagocytosis 34

2.6 Gentamicin survival E.coli phagocytosis assay 36

$\begin{array}{ll}2.7 \text { General Flow Cytometry } & 37\end{array}$

2.8 Cytospin and Light microscopy 38

2.9 ELISA: Enzyme Linked Immunosorbant Assay 39

2.10 Greiss Reaction $\quad 40$

2.11 Metabolism and Proliferation Assays (MTT assays) 40

2.12 Confocal Microscopy 42

2.13 Statistics 46

Chapter 3: Cytotoxic Effects of MSD on RAW264.7 and J774.2 Murine 47

Macrophages

3.1 Introduction $\quad 47$

3.2 Results $\quad 50$

3.3 Discussion $\quad 55$ 
Chapter 4: Effect of MSD on Receptor mediated endocytosis

4.1 Introduction $\quad 64$

$\begin{array}{ll}4.2 \text { Results } & 66\end{array}$

$\begin{array}{ll}4.3 \text { Discussion } & 73\end{array}$

Chapter 5: Bacterial Phagocytosis by MSD treated RAW264.7 macrophages 77

5.1 GFP-labelled E.coli phagocytosis by RAW264.7 macrophages 77

5.2 pHrodo E.coli bioparticles and phagocytosis by RAW264.7 89

Macrophages

5.3 Interactions between MSD and IFN- $\gamma$ during pHrodo phagocytosis 107

5.4 Use of Confocal microscopy to visualize pHrodo phagocytosis 113

Chapter 6: Bacterial Killing by MSD treated RAW264.7 macrophages $\quad 130$

6.1 Introduction 130

6.2 Results 133

6.3 Discussion 143

Chapter 7: Effects of MSD treatment on cytokine production during 151 bacterial killing in RAW264.7 macrophages

7.1 Interactions between TNF- $\alpha$ and paclitaxel in LPS stimulated 152

RAW264.7 macrophages

7.2 Changes in TNF- $\alpha$ and NO production during pHrodo phagocytosis 161 in MSD treated macrophages

7.3 Changes in cytokine production during bacterial killing in MSD $\quad 170$ treated macrophages

7.4 Effects of IFN- $\gamma$ pretreatment on TNF- $\alpha$ production in MSD treated macrophages during phagocytosis and killing

$\begin{array}{ll}\text { Chapter 8: General Discussion } & 190\end{array}$

8.1 Review of Overall Findings $\quad 190$

$\begin{array}{ll}\text { 8.2 Future Directions } & 194\end{array}$

8.3 Final Conclusion 196

Chapter 9: References $\quad 197$

$\begin{array}{ll}\text { Appendices } & 217\end{array}$ 
LIST OF TABLES AND FIGURES

\section{Chapter 1:}

Figure 1.1

Figure 1.2

Figure 1.3

\section{Chapter 2:}

Table 2.1

Chapter 3:

Figure 3.1.1

Figure 3.1.2

Figure 3.1.3

Table 3.1

Figure 3.1.4

Figure 3.1.5

\section{Chapter 4:}

Figure 4.1.1

Figure 4.1.2

Figure 4.1.3

Figure 4.1.4

Figure 4.1.5

\section{Chapter 5:}

Figure 5.1.1

Figure 5.1.2

Figure 5.1.3

Figure 5.1.4

Figure 5.1.5
Microtubule structure and polymerization.

Anti-mitotic drugs and their microtubule binding sites.

Macrophage endocytic processes.

Range of imaging parameters for confocal imaging with 3 colour dyes and non-confocal differential phase contrast imaging.

Representative $\mathrm{IC}_{50}$ curves for paclitaxel, docetaxel and peloruside $\mathrm{A}$ in RAW264.7 macrophages.

Representative $\mathrm{IC}_{50}$ curves for paclitaxel, docetaxel and peloruside A in J774.2 macrophages.

$\mathrm{IC}_{50}$ curves for paclitaxel, docetaxel and peloruside A in HL-60 cells. $\mathrm{IC}_{50}$ values for paclitaxel, docetaxel and peloruside A in RAW264.7, J774.2 and HL-60 cell lines.

MTT curves for paclitaxel at 2 and 8 hours in RAW264.7 cells. $\mathrm{OD}_{570}$ values from a 72 hour MTT assay on RAW264.7, J774.2 and HL60 cells.

Flow cytometry plots showing F4/80 and CD11b characterized RAW264.7 macrophages incubated with DiI labelled LDL particles. Dose dependent increases in receptor mediated endocytosis of DiI-LDL and DiI-ac-LDL by RAW264.7 macrophages.

Uptake of LDL or ac-LDL in RAW264.7 macrophages. Uptake of DiI-LDL and DiI-ac-LDL by RAW264.7 macrophages in the presence of paclitaxel.

Uptake of DiI-LDL or DiI-ac-LDL in paclitaxel-treated RAW264.7 macrophages.

Histogram and Flow cytometry plots showing GFP+ve and GFP-ve E.coli.

Flow cytometry plots showing effect of FACS buffer on GFP+ve E.coli.

Bacterial population is hard to distinguish from unstained macrophages, when bacteria are added at high MOI.

CMTMR labelled macrophages and GFP+ve bacteria.

RAW264.7 cells incubated with increasing MOI of GFP+ve bacteria. 
Figure 5.1.6 GFP+ve bacteria incubated with gentamicin are fluorescent and express a GFP+ve signal.

Figure 5.2.1 Increase in fluorescence of phagocytosed pHrodo E.coli bioparticles.

Figure 5.2.2 pHrodo particles show an increase in fluorescence when phagocytosed by macrophages.

Figure 5.2.3 Macrophages and pHrodo alone show low percent positive cells; however intensity of fluorescence is high.

Figure 5.2.4 Phagocytosis of pHrodo by MSD treated macrophages.

Figure 5.2.5 Changes in fluorescence intensity of cells positive for pHrodo uptake in the presence of MSD treatment.

Figure 5.2.6 Phagocytosis of pHrodo in the presence of (A) ixabepilone and (B) mycothiazole.

Figure 5.2.7 Changes in fluorescence intensity of cells positive for pHrodo uptake in the presence of ixabepilone and mycothiazole.

Figure 5.3.1 pHrodo phagocytosis by MSD treated macrophages is not affected, by presence or absence of IFN- $\gamma$ pre-treatment.

Figure 5.4.1 pHrodo particles fluoresce only when phagocytosed.

Figure 5.4.2 Phagocytosed pHrodo has a higher intensity of fluorescence as compared to external pHrodo.

Figure 5.4.3 Series of images showing pHrodo phagocytosis in CFSE and Hoechst stained cells.

Figure 5.4.4 Unstained cells phagocytose more pHrodo as compared to Hoechst and CFSE stained cells.

Figure 5.4.5 Time-lapse images showing internalization and acidification of pHrodo during phagocytosis by RAW264.7 cells.

Figure 5.4.6 Phagocytosis of pHrodo accompanied with increase in fluorescence over time in untreated and paclitaxel treated cells, while colchicine and latrunculin A treated cells show reduced or no phagocytosis respectively.

Figure 5.4.7 Vehicle treated macrophages phagocytose pHrodo similarly to untreated macrophages.

Figure 5.4.8 Colchicine and Latrunculin A treated cells show a significant decrease in phagocytic ability, while paclitaxel treated cells show altered phagocytosis patterns.

Figure 5.4.9 Colchicine and Latrunculin A treated cells show a significant decrease in phagocytic ability, while paclitaxel treated cells show alterations in initial phagocytic patterns. 


\section{Chapter 6:}

Figure 6.1.1

Figure 6.2.1

Figure 6.2.2

Figure 6.2.3

Figure 6.2.4

Figure 6.2.5

Figure 6.2.6

Figure 6.2.7

\section{Chapter 7:}

Figure 7.1.1

Figure 7.1.2

Figure 7.1.3

Figure 7.1.4

Figure 7.2.1

Figure 7.2.2

Figure 7.2.3

Figure 7.3.1

Figure 7.3.2

Figure 7.3.3

Figure 7.3.4
Plating of bacteria after gentamicin treatment enables determination of macrophage bactericidal activity.

Phagocytosis of bacteria in RAW264.7 macrophages at varying MOI. Effect of opsonisation on phagocytosis of bacteria at MOI 25:1 in J774.2 and RAW264.7 macrophages.

Confirmation of E.coli internalization by RAW264.7 and J774.2 macrophages.

RAW264.7 macrophages phagocytose similar levels of bacteria irrespective of IFN- $\gamma$ pre-treatment or bacterial opsonisation.

Bacterial survival in RAW264.7 macrophages treated with high and low dose MSD.

Bacterial survival in RAW264.7 macrophages treated with low and high dose MSD.

Effects of IFN- $\gamma$ pre-treatment on bacterial killing by MSD treated RAW264.7 macrophages.

TNF- $\alpha$ production in 2 hour LPS-stimulated RAW264.7 and J774.2 macrophages and 8 hour LPS-stimulated RAW264.7 and J774.2 macrophages.

RAW264.7 and J774.2 cells exhibit a constant rate of metabolism in the presence of 2 hours or 8 hours LPS-stimulation.

Effect of paclitaxel on TNF- $\alpha$ production from 2 hours and 8 hours LPS stimulated RAW264.7 macrophages.

Rate of metabolism in paclitaxel treated, LPS stimulated RAW264.7 cells at 2 hours and 8 hours.

Drug treatment during pHrodo phagocytosis does not alter TNF- $\alpha$ production in RAW264.7 macrophages.

Drug treatment during pHrodo phagocytosis does not alter TNF- $\alpha$ production in RAW264.7 macrophages.

Treatment with high dose MSD affects nitric oxide production during phagocytosis of bacteria.

Changes in TNF- $\alpha$ production by MSD treated macrophages during bacterial phagocytosis.

Effect of zampanolide, ixabepilone and mycothiazole on TNF- $\alpha$ production during bacterial killing.

NO production is not significantly affected by MSD treatment at 1 hour or 3 hours during bacterial killing.

NO production is not significantly affected by MSD or mycothiazole treatment at 1 hour or 3 hours during bacterial killing. 
Figure 7.3.5 At 3 hours, Il-12 production is variable during bacterial killing in treated macrophages.

Figure 7.4.1 Absence or presence of IFN- $\gamma$ pre-treatment alters TNF- $\alpha$ production during bacterial killing in drug treated RAW264.7 macrophages.

Figure 7.4.2 Absence or presence of IFN- $\gamma$ pre-treatment alters TNF- $\alpha$ production during phagocytosis in drug treated RAW264.7 macrophages.

Chapter 8:

No figures or tables. 


\section{ABBREVIATIONS}

\begin{tabular}{|c|c|}
\hline $\begin{array}{l}\mathrm{ABC} \\
\text { ac-LDL }\end{array}$ & $\begin{array}{l}\text { ATP-binding cassette } \\
\text { acetylated low density lipoprotein }\end{array}$ \\
\hline ANOVA & Analysis of Variance \\
\hline ATCC & American Type Cell culture \\
\hline BMDM & bone marrow derived macrophages \\
\hline CFDA-SE-(CFSE) & 5,6-carboxyfluorescein succinimidyl ester \\
\hline $\mathrm{CFU}$ & colony forming units \\
\hline CMTMR & Cell Tracker Orange \\
\hline $\mathrm{CR}$ & complement receptor \\
\hline CTCM & complete T-cell medium \\
\hline CTL & cytotoxic lymphocytes \\
\hline Сус & Cychrome \\
\hline DC & dendritic cells \\
\hline DD & death domain \\
\hline $\mathrm{DIC}$ & Differential Interference Contrast \\
\hline DiI & $\begin{array}{l}\text { 1,1'-dioctadecyl-3,3,3',3'-tetramethyl- } \\
\text { indocarbocyanineperchlorate }\end{array}$ \\
\hline DMEM & Dulbecco's modified eagle medium \\
\hline DMSO & dimethly sulfoxide \\
\hline EAE & experimental autoimmune encephalomyelitis \\
\hline ELISA & Enzyme-linked Immunosorbant Assay \\
\hline FcR & Fc Receptor \\
\hline FCS & Fetal calf serum, heat in-activated \\
\hline Fitc & Fluorescein Isothiocyanate \\
\hline GFP & green fluorescent protein \\
\hline GM-CSF & granulocyte macrophage colony stimulating factor \\
\hline HBSS & Hank’s Buffered Saline Solution \\
\hline IFN & interferon \\
\hline $\operatorname{IgG}$ & Immunoglobulin $\mathrm{G}$ \\
\hline IL & interleukin \\
\hline iNOS & inducible nitric oxide synthase \\
\hline IPTG & isopropyl $\beta$-D-1-thiogalactopyranoside \\
\hline ITAM & immunoreceptor tyrosine-based activation motif \\
\hline ITIM & immunoreceptor tyrosine-based inhibitory motif \\
\hline Ixabepilone & Ixempra® \\
\hline JNK & Jun N-terminal kinase \\
\hline LB media & Luria Bertani media \\
\hline LDL & low density lipoprotein \\
\hline LPS & bacterial lipopolysaccharide \\
\hline
\end{tabular}




$\begin{array}{ll}\text { LTA } & \text { lipoteichoic acid } \\ \text { MAP } & \text { microtubule associated protein } \\ \text { MAPK } & \text { mitogen activated protein kinase } \\ \text { MDD } & \text { microtubule destabilizing drugs } \\ \text { MDR } & \text { multi-drug resistance } \\ \text { MFI } & \text { mean fluorescence intensity } \\ \text { MHC } & \text { Major Histocompatibility complex } \\ \text { MOI } & \text { multiplicity of infection } \\ \text { MSD } & \text { microtubule stabilizing drugs } \\ \text { MTA } & \text { microtubule targeting agents } \\ \text { MTOC } & \text { Microtubule organizing centre } \\ \text { MTT } & \text { 3-(4,5-dimethylthiazoyl-2-yl)-2,5-diphenyltetrazoliumbromide } \\ \text { NaNO } & \text { Sodium nitrite } \\ \text { NF- } \text { BB } & \text { nuclear factor -Kappa B } \\ \text { NO } & \text { nitric oxide } \\ \text { NOS } & \text { nitric oxide synthase } \\ \text { OD } & \text { optical density } \\ \text { OD } & \text { optical density } \\ \text { PAMPS } & \text { pathogen associated molecular patterns } \\ \text { PBS } & \text { Phosphate Buffered Saline } \\ \text { P-gp } & \text { P-glycoprotein } \\ \text { PMA } & \text { phorbol 12-myristate 13-acetate } \\ \text { PRR } & \text { pattern recognition receptors } \\ \text { RME } & \text { receptor mediated endocytosis } \\ \text { ROS } & \text { reactive oxygen species } \\ \text { SA-HRP } & \text { Streptavadin-Horse-Radish-Peroxidase } \\ \text { SDS } & \text { sodium dodecyl sulphate } \\ \text { SEM } & \text { Standard error mean } \\ \text { SLE } & \text { systemic lupus erythematosus } \\ \text { SR } & \text { scavenger receptor } \\ \text { SRBC } & \text { sheep red blood cells } \\ \text { STAT } & \text { signal transducers and activators of transcription } \\ \text { Taxol } & \text { Taxol@ } \\ \text { TBH } & \text { tumor bearing host } \\ \text { Th1 or Th2 } & \text { T- helper 1 or T-helper 2 } \\ \text { TLR } & \text { Toll like receptor } \\ \text { TNFR } & \\ \text { TNF- } \alpha & \end{array}$




\section{CHAPTER 1: INTRODUCTION}

\subsection{Microtubules and Microtubule targeting agents:}

\subsubsection{Microtubule structure and dynamics}

Microtubules are extremely important in cell division and mitosis, which makes them an effective target for anti-cancer drugs (Jordan et al., 2004; Risinger et al., 2008). Compounds that target microtubules and inhibit their normal function have proven to be one of the best classes of cancer chemotherapeutic drugs to date (Zhou et al., 2005). One of the reasons for the effectiveness of these compounds is that cancer cells divide more frequently than normal cells, and they do not respond to cell cycle checkpoints. Additionally, spindle microtubules are $10-100$ fold more dynamic than cytoplasmic microtubules (Jordan et al., 2004; Jordan et al., 2007). Therefore these cells are more vulnerable to mitotic poisons.

Microtubules are dynamic polymers composed of $\alpha \beta$-tubulin dimers and along with actin and intermediate filaments, make up the cytoskeleton. Microtubules are found in all dividing and interphase eukaryotic cells (Desai et al., 1997; Jordan et al., 2004; Risinger et $a l .$, 2008). They have diverse roles in mitosis, cell motility, intracellular transport, secretion and maintenance of cell shape and polarity (Downing et al., 1998a; Conde et al., 2009). Microtubules form when $\alpha$ - and $\beta$ - tubulin associate into heterodimers, which then associate in a head to tail fashion to form linear protofilaments. Typically thirteen protofilaments associate in parallel, but axially offset to form the hollow $24 \mathrm{~nm}$ wide cylindrical microtubule (Fig 1.1A) (Desai et al., 1997; Risinger et al., 2008; Conde et al., 2009). Microtubules are polar structures, with a plus end and a minus end, due to the pattern in which the subunits assemble. Within eukaryotic cells, the minus ends of microtubules are anchored and stabilized at the centrosome or microtubule organizing 
centre (MTOC) via $\gamma$-tubulin (Li et al., 1995; Howard et al., 2003). Interaction of the minus end with $\gamma$-tubulin of the MTOC occurs via $\alpha$-tubulin and $\beta$-tubulin remains exposed at the plus end (Nogales, 2000). Thus, the microtubule plus ends extend towards the cell periphery and consequently all addition and loss of subunits occurs at the plus end (Risinger et al., 2008). Polymerization rates are also more rapid and extensive at the plus end (Desai et al., 1997; Downing et al., 1998a; Nogales et al., 1999).

The tubulin heterodimer contains two GTP-binding sites, an exchangeable site (Esite) on $\beta$-tubulin and a non-exchangeable site (N-site) on $\alpha$-tubulin (Gelfand et al., 1991; Desai et al., 1997). Each tubulin monomer binds GTP, however GTP-binding at the N-site is non-exchangeable, while GTP-binding at the E-site is exchangeable (Downing et al., 1998b; Nogales, 2000). Assembly of tubulin subunits into protofilaments is promoted by binding of GTP to E-sites on $\beta$-tubulin, which enables polymerization (Cassimeris et al., 1987; Gelfand et al., 1991; Nogales, 2000). After polymerization, GTP hydrolyzes to GDP and becomes non-exchangeable, resulting in a microtubule body of GDP bound tubulin and a layer of GTP bound tubulin capping the top of the microtubule (Fig 1.1B) (Cassimeris $e t$ al., 1987; Gelfand et al., 1991; Downing et al., 1998b; Nogales, 2000). E-site GDP is not exchanged for GTP when in the microtubule polymer, but if the GTP cap is lost and depolymerisation occurs, the free tubulin dimers can then exchange E-site GDP for GTP (Cassimeris et al., 1987; Desai et al., 1997). This unique pattern of GTP binding and hydrolysis seen on $\alpha$ - and $\beta$-tubulin leads to non-equilibrium behaviour of microtubules, known as dynamic instability (Cassimeris et al., 1987; Desai et al., 1997; Nogales, 2000).

During dynamic instability, the microtubules alternate stochastic switching of the plus end between long periods of slow lengthening and brief periods of rapid shortening (Jordan et al., 2004). Dynamic instability is characterized by four main variables: the rate of 
microtubule growth, the rate of shortening, the frequency of switching between growth and shortening (called catastrophe) and the frequency of switching between shortening and growth (called rescue) (Fig. 1.1B) (Jordan et al., 2004; Risinger et al., 2008). Another dynamic behaviour displayed by microtubules is called "treadmilling". This behaviour is characterized by a net flow of subunits from the plus end to the minus end, with no significant change in microtubule mass or length (Desai et al., 1997; Nogales, 2000; Jordan et al., 2004; Risinger et al., 2008). Dynamic instability and treadmilling are compatible processes (Nogales, 2000; Jordan et al., 2004), and a population of microtubules can exhibit primarily treadmilling behaviour, dynamic instability behaviour or a combination of both (Jordan et al., 2004). Tubulin isotypes, the extent of post-translational modifications and the presence of regulatory proteins determine the dynamic behaviour that a population of microtubules display (Desai et al., 1997; Jordan et al., 2004). 
Figure 1.1

A

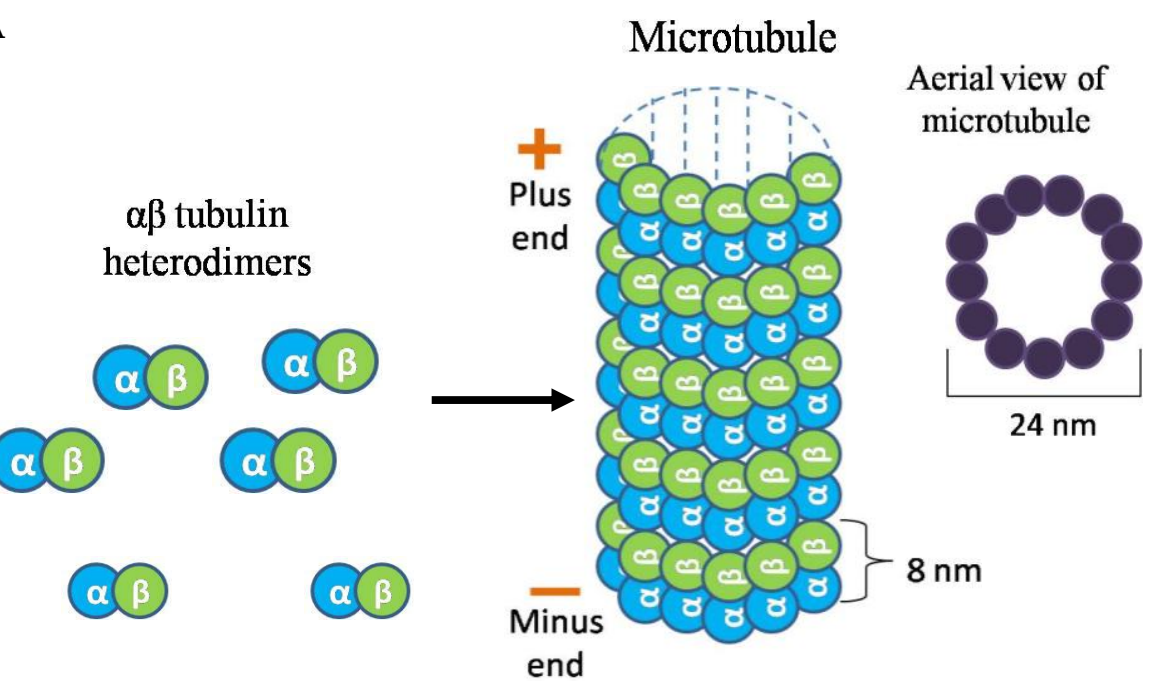

B

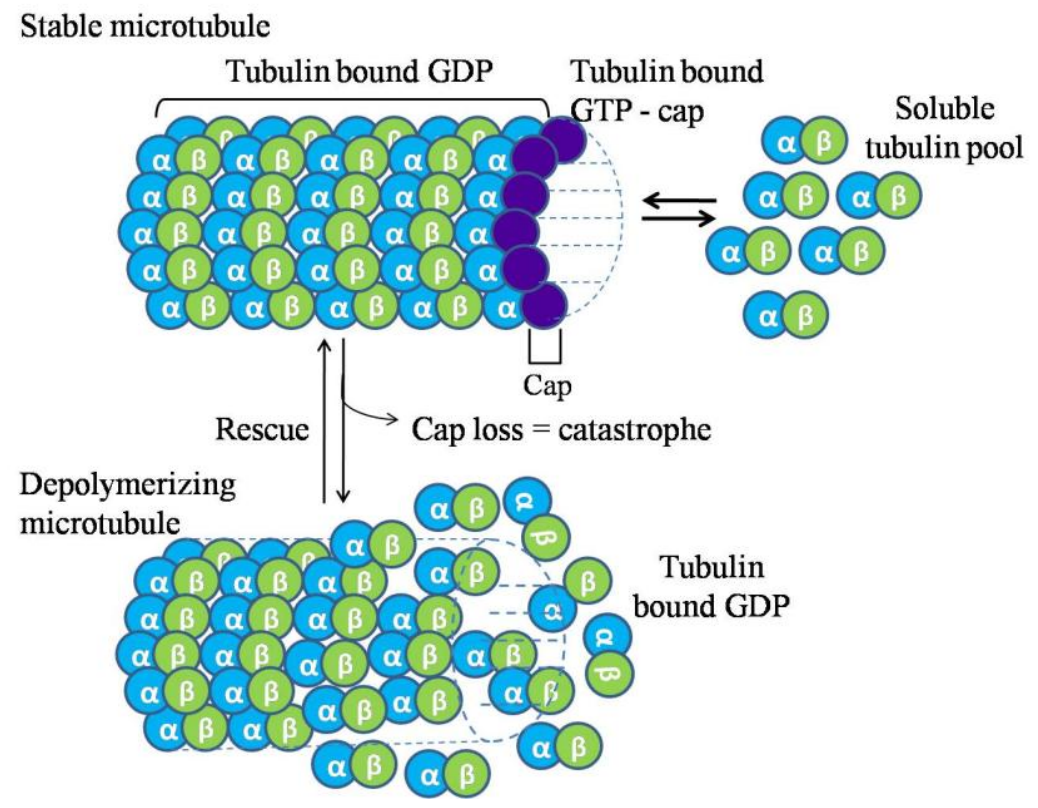

Figure 1.1 Microtubule structure and polymerization.

A: $\alpha \beta$ tubulin heterodimers associate to form protofilaments. Typically, 13 protofilaments associate parallel to each other to form the microtubule. Microtubules have a plus end and a minus end and are $24 \mathrm{~nm}$ in diameter while each heterodimer layer is $8 \mathrm{~nm}$ in diameter.

B: Presence of a GTP-bound tubulin cap stabilizes the microtubule, but loss of this cap leads to catastrophe and microtubule destabilization.

This figure was adapted from Jordan and Wilson, 2004. Nature Reviews Cancer. 
Dynamic instability is important for microtubule function. It allows microtubules to rapidly reorganize the cytoskeleton and adopt spatial arrangements in response to cellular need or mechanical work (Desai et al., 1997). This is especially important during transition between mitosis and interphase stages of the cell cycle (Cassimeris et al., 1987). Various cellular processes, such as the intracellular transport of proteins and other cargo across the cell, cell polarization, adhesion, cell migration and morphogenesis depend on microtubules and the maintenance of microtubule dynamics (Cassimeris et al., 1987; Kelly, 1990; Jordan et al., 2008). The motor proteins kinesin and dynenin depend on microtubule and actin scaffolding to carry out transport functions (Rogers et al., 2000; Apodaca, 2001). Microtubules are also involved in the organization of the cytoskeleton itself. For example, organization of actin filaments during cell migration is achieved by transport of the actin components along the microtubule network (Rogers et al., 2000). Polymerization dynamics of microtubules are central to their biological function and altering these dynamics has impacts on physiological and cellular function.

\subsubsection{Microtubule destabilizing drugs (MDDs)}

Microtubule targeting agents (MTA) inhibit mitosis by blocking proliferation in the $\mathrm{G}_{2} / \mathrm{M}$ phase, causing the damaged cells to undergo apoptosis (Jordan et al., 2004; Jordan et al., 2007). MTA have been isolated from natural as well as synthetic sources and include the clinically important drugs paclitaxel (Taxol), docetaxel (Taxotere) and the vinca alkaloids (vincristine and vinblastine), as well as epothilones, discodermolide, nocodazole, and colchicine (Sorger et al., 1997). Based upon their effects on microtubules, MTAs are divided into two classes of compounds - the microtubule destabilizing drugs (MDD), and the microtubule stabilizing drugs (MSD). Drugs from both classes are currently used 
clinically to treat lymphomas, leukemia and solid tumors (Jordan et al., 2004; Jordan et al., 2007).

Microtubule destabilizing drugs or MDDs, as their name suggests, interact with microtubules causing destabilization of protofilaments, inhibition of polymerization and an overall decrease in polymer mass (Jordan et al., 2007; Risinger et al., 2008). MDDs include compounds such as the Vinca alkaloids (vinblastine, vincristine, vinorelbine), cryptophycins, halichondrins, colchicine and combretastatins (Jordan et al., 2004). Since these compounds bind to microtubule and alter dynamics and function, they have been successful as anti-cancer chemotherapeutics (Risinger et al., 2008). A large majority of MDDs bind microtubules in either of two domains, the vinca domain or the colchicine domain (Fig 1.2). The Vinca alkaloids and halichondrins bind to the vinca domain, while colchicine, 2-methoxyestradiol (2ME2) and combretastatins bind to the colchicine domain (Jordan et al., 2004; Risinger et al., 2008).

\subsubsection{Microtubule destabilizing agent - Colchicine}

Colchicine is a naturally occurring MDD isolated from the meadow saffron Colchicum autmnale (Zhou et al., 2005). It is one of the earliest MTAs identified and its mechanism of action has been extensively investigated (Zhou et al., 2005; Correia et al., 2008). Colchicine exhibits potent anti-mitotic, anti-inflammatory and anti-fibrotic properties, and is used in the treatment of gout (Jordan et al., 2004; Bhattacharyya et al., 2008). Colchicine has also been used successfully in the treatment of autoinflammatory diseases such as familial Mediterranean fever (FMF) (Bhattacharyya et al., 2008). Although colchicine is a potent microtubule depolymerizer with anti-mitotic properties, its development as a chemotherapeutic is hampered due to the severe toxicities that 
accompany its use at doses required for therapeutic effects (Jordan et al., 2004; Morris et al., 2008; Risinger et al., 2008).

Colchicine binds microtubules at the interface of the $\alpha / \beta$ tubulin heterodimer (Risinger et al., 2008). It preferentially binds to unpolymerized tubulin heterodimers in solution and forms a stable complex that inhibits microtubule dynamics upon addition to microtubule ends (Fig 1.2) (Jordan et al., 2004; Risinger et al., 2008). While high dose colchicine is toxic, at lower doses it displays immunomodulating properties that make it useful in the treatment of gout (Jordan et al., 2004; Risinger et al., 2008). Other agents that bind in the colchicine domain, such as combretastatin, are currently being investigated in clinical trials against solid tumors, to validate these agents as chemotherapeutics (Jordan et al., 2004; Schmidt et al., 2007; Risinger et al., 2008) . 


\section{Figure 1.2}

A

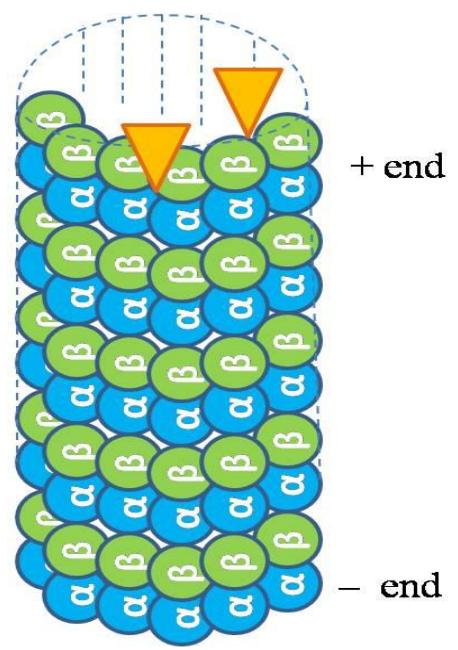

B

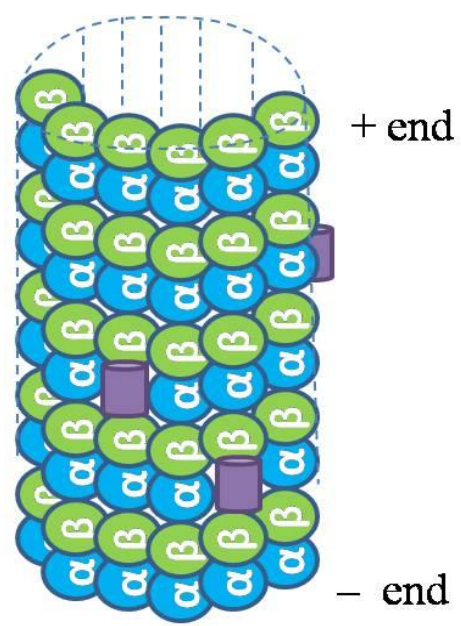

C

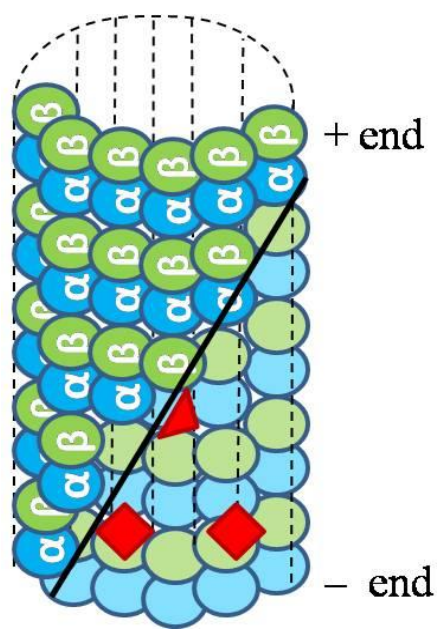

\section{Vinblastine colchicine $\checkmark$ paclitaxel}

Figure 1.2 Anti-mitotic drugs and their microtubule binding sites.

A: Vinca alkaloid binding site: Vinblastine molecules bind to high-affinity sites at the plus-end of the microtubule.

B: Colchicine binding site: colchicine molecules form a complex with tubulin dimers at the interface of $\alpha / \beta$ tubulin heterodimers and copolymerize into the microtubule lattice.

C: Taxane binding site: microtubule cut away to view interior surface; paclitaxel binds along the inside surface of the microtubule.

This figure was adapted from Jordan and Wilson, 2004. Nature Reviews Cancer 


\subsubsection{Microtubule stabilizing drugs (MSDs)}

Microtubule stabilizing drugs or MSDs, as the name suggests, interact with microtubules causing their stabilization and increasing the polymer mass. MSD include drugs such as paclitaxel, docetaxel, the epothilones, peloruside A, laulimalide, the sarcodictyins and the eleutherobins. MSDs can affect dividing cells as well as non-dividing or interphase cells and their effects vary depending on the stage of cell cycle (Jordan et al., 1993; Altmann, 2001; Hood et al., 2002). MSDs such as paclitaxel, docetaxel and epothilones are currently used clinically as chemotherapeutics, while analogues of these compounds are in single or combination phase I, II or III clinical trials (Jordan et al., 2007) . While the classification of MTAs as "stabilizers" or "destabilizers" is simple and useful, it is also slightly misleading. At high concentrations, MDDs cause destabilization and a decrease in polymer mass, while MSDs cause stabilization and an increase in polymer mass (Jordan et al., 2004; Risinger et al., 2008). However at lower, clinically relevant doses, both groups potently suppress microtubule dynamics (Jordan et al., 2007). Since microtubule dynamics are extremely sensitive to regulation, at low doses both MDDs and MSDs can kinetically stabilize microtubules without changing the polymer mass. Thus at a very basic level and at low concentrations, both MSD and MDD act as mitotic inhibitors. Due to this, their effects on microtubule dynamics are more powerful than their effects on polymerization or depolymerization (Jordan et al., 2004; Jordan et al., 2007).

While it is known that MSDs have cytotoxic effects on dividing cells, (Hood et al., 2002; Gaitanos et al., 2004) little is known about their effects in interphase cells. MSD treatment in interphase cells leads to appearance of multiple micronuclei and microtubule bundling resulting in functional disturbance (Jordan et al., 1993; Hood et al., 2002). While microtubule actives have enjoyed great clinical success, there are significant difficulties 
associated with their use. These in particular are related to toxicity and their limited effectiveness in some cancers due to multi-drug resistance. Consequently, new compounds that target microtubules in similar ways and are effective in multi-drug resistant cancers are continuously being sought. MSDs and their effects are discussed in more detail in the sections below.

\subsubsection{Microtubule stabilizing agent - paclitaxel}

Paclitaxel, isolated from the bark of the Pacific yew tree, taxus brevifolia, belongs to the Taxane group of anti-mitotic agents (Wani et al., 1971; Chan et al., 2000). It was the first identified microtubule stabilizer and is used clinically to treat solid tumors of the breast, ovaries and lung, as well as in combination therapies with different agents to treat head and neck and gastroesophageal cancers (Rowinsky et al., 1995; Spratlin et al., 2007; Morris et al., 2008). At low nanomolar concentrations, paclitaxel is capable of stabilizing microtubules and promoting their polymerized form. However these microtubules are dysfunctional, leading to apoptosis and cell cycle arrest in the G2/M phase of the cell cycle (Panchagnula, 1998). paclitaxel is also able to induce abnormal spindle morphologies, causing spindles to lose organization and aggregate into ball-shaped masses with condensed chromosomes and multiple asters (Jordan et al., 1993). In addition to causing mitotic block, low doses of paclitaxel induce microtubule bundling in interphase cells (Jordan et al., 1993; Risinger et al., 2008). Microtubule bundling affects cellular function and processes dependent on dynamic microtubules. This bundling effect could also contribute to the antiproliferative activity of paclitaxel (Jordan et al., 1993).

While paclitaxel is an effective chemotherapeutic, it is not without its drawbacks, particularly hypersensitivity and toxic side effects. The principal toxic effects associated 
with paclitaxel treatment were neutropenia, gastro-intestinal disturbances, alopecia, cardiac effects and neurotoxicity (Rowinsky et al., 1995). Paclitaxel was also susceptible to development of resistance by multi-drug resistance transporters such as $\mathrm{P}$-glycoprotein (Pgp) (Risinger et al., 2008). A major concern with paclitaxel treatment was the development of type I hypersensitivity reactions in reaction to paclitaxel's vehicle, polyoxyethylated castor oil (Cremophor EL) (Rowinsky et al., 1995). Due to paclitaxel's hydrophobicity, and limited aqueous solubility, it is necessary to use Cremophor EL for drug delivery to cells (Rowinsky et al., 1995; Gelderblom et al., 2001). This solves solubility concerns, but can cause severe hypersensitivity reactions (Panchagnula, 1998; Gelderblom et al., 2001; Spratlin et al., 2007). This problem was partially overcome by pre-medicating with corticosteroids and histamine $\mathrm{H}_{1}$ and $\mathrm{H}_{2}$ antagonists, which caused the incidence of hypersensitivity to drop (Rowinsky et al., 1995). Several anti-mitotic agents and MSD with fewer side effects and increased efficacy are currently under development to overcome the above mentioned limitations.

\subsubsection{Microtubule stabilizing agent - docetaxel}

Docetaxel is a second generation taxane derived from the needles of the European Yew tree (Herbst et al., 2003). It is a semi-synthetic analogue of paclitaxel and is a more potent MSD as compared to paclitaxel (Herbst et al., 2003; Si et al., 2003). Docetaxel has unique properties to paclitaxel as it exhibits linear pharmacokinetics and is retained intracellularly for a longer period than paclitaxel due to differences in drug efflux (Herbst et al., 2003). Docetaxel is currently used in treating a variety of tumors, including breast, prostate and head and neck cancers, in combination as well as in mono-therapies (Herbst et al., 2003; Zhou et al., 2005). Docetaxel can be administered without Cremophor EL and 
while side effects such as neutropenia, neuropathy and immunosuppression are common, these are to a much lesser extent as compared to paclitaxel.

\subsubsection{Microtubule stabilizing agent - peloruside A}

A novel MSD, peloruside A was isolated in 2000 from the New Zealand marine sponge Mycale hentscheli (West et al., 2000). Similarly to paclitaxel, peloruside A binds to polymerized tubulin and blocks mitosis at the G2/M checkpoint (Hood et al., 2002). Peloruside A can also cause microtubule bundling in a manner similar to paclitaxel (Hood et al., 2002; Crume et al., 2007). The interest generated by peloruside A is due to the fact that it is cytotoxic in the paclitaxel resistant cell lines. Additionally, its tubulin binding site is distinct to that of the taxanes, suggesting that peloruside $\mathrm{A}$ and the taxanes could be used synergistically (Hood et al., 2002; Gaitanos et al., 2004; Wilmes et al., 2007). Peloruside A is currently being developed for clinical use and it has promising advantages over the existing taxanes and other agents, however studies involving peloruside A are restricted due to its limited supply.

Docetaxel, and paclitaxel, are among some of the most effective anti-cancer drugs introduced in the last ten years for the treatment of breast and ovarian cancer. These drugs not only exhibit potent anti-cancer activity but also have the potential to be used as therapeutics in hyper-proliferative autoimmune diseases. Understanding the immunomodulatory effects of existing MSDs along with newly discovered MSDs will improve their use as chemotherapeutics as well as extend their potential applications beyond oncology. 


\subsection{Macrophage Functions and Immune Responsiveness}

\subsubsection{Macrophage Function: General role in immunity}

Macrophages are cells of the innate immune system that are specialized in phagocytosis and pathogen killing. Macrophages are also involved in other processes such as antigen presentation to T-cells, activation of the adaptive immune system, tissue remodeling and wound repair, as well as defense against microbial invasion and recognition and killing of tumor cells (Klimp et al., 2002; Mukhopadhyay et al., 2004). Macrophages carry out their functions via phagocytosis and killing, or via the release of reactive oxygen species (ROS) and cytokines such as tumor necrosis factor $-\alpha(\mathrm{TNF}-\alpha)$ and interleukin (IL)-12, IL-6 and IL-8 (Klimp et al., 2002; Fujihara et al., 2003).

Macrophages respond to pathogens via pattern-recognition receptors (PRRs) that recognize pathogen associated molecular patterns (PAMPs) (Aderem et al., 1999). PAMPSs are evolutionary conserved pathogen motifs, such as dsRNA, lipopolysaccharide (LPS), and flagellin (Taylor et al., 2005). Macrophages express a variety of cell surface receptors to aid in particle recognition and internalization. Some of these receptors are involved in transmitting signals for initiation of phagocytes, while others increase binding and internalization efficiency (Aderem et al., 1999; Underhill et al., 2002). Phagocytic receptors include Fc receptors (FcR) such as FcyRI (CD64), Fc $\gamma$ RIII (CD16), complement receptors (CR) such as CR1 and CR3 (CD11b, Mac1), scavenger receptors (SR) such as SRA, and Toll-like receptors (TLR) such as TLR2 and TLR4 (Aderem et al., 1999; Underhill et al., 2002; Mukhopadhyay et al., 2004). When faced with a pathogenic challenge, macrophages not only carry out phagocytosis but also secrete proinflammatory cytokines. LPS from gram negative bacteria activates macrophages via TLR-4 and initiates the secretion of TNF- $\alpha$, interleukin (IL)-1, IL-6, IL-8 and IL-12 (Ding et al., 1990b; 
Dobrovolskaia et al., 2002; Fujihara et al., 2003). Macrophages also secrete other proinflammatory mediators such as nitric oxide (NO) and reactive oxygen species (ROS) in response to bacterial stimuli.

\subsubsection{Fcy Receptors and complement receptors}

Opsonization or coating of pathogens with complement proteins or IgG antibodies stimulates phagocytosis via the CRs or the FcRs respectively (Aderem et al., 1999; Underhill et al., 2002). IgG opsonized particles can be recognized and phagocytosed by Fc $\gamma$ Rs. Macrophages express a combination of low affinity receptors (Fc $\gamma$ RIIA and Fc $\gamma$ RIIIA) and high affinity receptors (Fc $\gamma \mathrm{RI})$ so IgG-opsonized particles are recognized simultaneously, increasing internalization (Underhill et al., 2002). Fc $\gamma$ Rs contain a stimulatory immunoreceptor tyrosine-based activation motif (ITAM) that activates phosphorylation cascades, or an immunoreceptor tyrosine-based inhibition motif (ITIM) that inhibits signaling (Aderem et al., 1999; Underhill et al., 2002).

In contrast, phagocytosis via CRs occurs when complement proteins in the serum opsonize particles via an antibody-dependent or-independent mechanism (Underhill et al., 2002). Complement opsonized particles are internalized via specific CRs, which include CR1, CR3 (CD11b or Mac1), and CR4 (Aderem et al., 1999). While Fc $\gamma \mathrm{R}$ are constitutively active for phagocytosis, CRs need additional signals in order to internalize complement-opsonized particles (Aderem et al., 1999; Underhill et al., 2002). In vitro this activation can be stimulated with phorbol 12-myristate 13-acetate (PMA), inflammatory cytokines such as TNF- $\alpha$, microbial products such as LPS as well as by granulocyte macrophage colony stimulating factor (GM-CSF) (Aderem et al., 1999; Underhill et al., 2002). 
While both FcyR- and complement-mediated phagocytosis require actin polymerization at the internalization site, the process by which these IgG- and complement-opsonized particles are internalized differ (Aderem et al., 1999). Fc $\gamma \mathrm{R}-$ mediated phagocytosis is an active process where "veils" of membrane rise around the particle and ingest it into the macrophage, while CR-mediated phagocytosis is more passive and particles "sink" into the cell with little to no formation of pseudopodia (Kaplan, 1977; Allen et al., 1996; Aderem et al., 1999). Using these different processes of uptake, macrophage receptors are capable of functioning synergistically to enhance internalization. When particles are coated with sub-optimal levels of IgG that would normally lower phagocytic efficiency, $\mathrm{CR}$ and $\mathrm{Fc} \gamma \mathrm{Rs}$ are able to coordinate ligation to enhance phagocytosis and produce synergistic effects of internalization (Ehlenberger et al., 1977).

\subsubsection{Scavenger receptors}

Scavenger receptors (SR) were first identified by Brown and Goldstein during their studies of low density lipoprotein (LDL) accumulation in atherosclerotic plaques (Goldstein et al., 1979). SR are involved in a wide range of functions, from receptor mediated endocytosis (discussed below), to host defense against bacteria, apoptotic cell phagocytosis, as well as cell adhesion (Peiser et al., 2000; Peiser et al., 2002). SR bind a range of ligands such as LDL, polyribonucleotides and silica particles and are classified into six different classes (A-F) according to their multi-domain structure (Mukhopadhyay et al., 2004).

SR-A has three spliced variants, SR-AI/II and SR-AIII, where SR-AIII is nonfunctional (Peiser et al., 2002; Mukhopadhyay et al., 2004). SR-A recognizes the lipid A moiety of LPS, the lipoteichoic acid (LTA) of gram positive bacteria, as well as bacterial CpG DNA. In studies characterizing the role of SR-A in bacterial phagocytosis, SR-A ${ }^{-/-}$ 
BMDM (bone-marrow-derived macrophages) from mice ingested fewer E.coli compared to wild-type cells (Peiser et al., 2000). The SR-A ${ }^{-1}$ mice also showed increased susceptibility to endotoxic shock and bacterial infections, due to impaired clearance of LPS and microorganisms (Peiser et al., 2000), thus confirming the role of SR-A in binding and ingestion of microbes.

\subsubsection{Macrophage function: endocytosis}

Endocytosis collectively refers to the processes by which cells internalize small molecules, macromolecules, and particles (Mukherjee et al., 1997). Phagocytosis, pinocytosis, clathrin-dependent receptor-mediated endocytosis and clathrin-independent receptor-mediated endocytosis all fall under the endocytosis umbrella. Endocytosis is important in processes such as regulation of cell surface receptor expression, cholesterol homeostasis and maintenance of cell polarity (Mukherjee et al., 1997). Clathrin-dependent receptor-mediated endocytosis and phagocytosis are the two main endocytic processes used by macrophages to endocytose pathogens and particles (Aderem et al., 1999). Receptor mediated endocytosis (RME) is the uptake of macromolecules and small particles $(<0.2$ $\mu \mathrm{m})$ and occurs via a clathrin based, actin-independent mechanism, while phagocytosis is the uptake of large particles $(>0.5 \mu \mathrm{m})$ and is actin-dependent and clathrin-independent (Brown et al., 1979b; Mellman, 1996; Mukherjee et al., 1997; Aderem et al., 1999).

\subsubsection{Macrophage function: Receptor mediated endocytosis}

The receptor mediated endocytic pathway was first studied using the lipoprotein receptor system and since then has come to be regarded as the prototype for receptor mediated studies (Brown et al., 1979b; Goldstein et al., 1979). Lipoprotein particles are 
cholesterol carriers that can be transported in the plasma and carried to target tissues. In humans the major cholesterol carrier is low density lipoprotein (LDL), which is taken up into macrophages via receptor mediated endocytosis (Brown et al., 1979b; Brown et al., 1983). Normal tissue macrophages from mice or other species, express few if any receptors for native LDL (Brown et al., 1979a; Brown et al., 1979b; Goldstein et al., 1979; Brown et al., 1983). Macrophages in vitro only take up minimal amounts of LDL and the cellular cholesterol content does not increase (Brown et al., 1983). However chemically modified LDL, such as acetylated LDL (ac-LDL) is taken up with high efficiency and results in massive cholesterol accumulation (Brown et al., 1979a; Goldstein et al., 1979).

Receptors that function in the uptake of native LDL are the LDL receptor, CD36 and SR-B1, while SR-A and -BI are involved in acetylated-LDL uptake (Brown et al., 1979a; Goldstein et al., 1979; Plüddemann et al., 2007). Receptor mediated endocytosis occurs via clathrin coated pits, where LDL binds to its receptor and the plasma membrane invaginates to form a coated vesicle which is trafficked to the lysosome. After fusing with the lysosome, the receptors are recycled to the plasma membrane and LDL is degraded (Fig 1.3) (Anderson et al., 1977; Anderson et al., 1982).

\subsubsection{Macrophage function: phagocytosis}

In contrast to receptor mediated endocytosis, phagocytosis is a largely actindependent process. However, only initial phagocytic events are actin-dependent. Once actin polymerizes at the phagocytic cup and the phagosome forms, actin is shed and phagosome maturation occurs along the endocytic pathway which is microtubule-dependent (Desjardins et al., 1994a; Aderem et al., 1999; Peachman et al., 2004). Phagocytosis 
involves a higher level of antigen processing and presentation than RME and depends on a functional and coordinated actin and microtubule cytoskeleton (Peachman et al., 2004). 


\section{Figure 1.3}

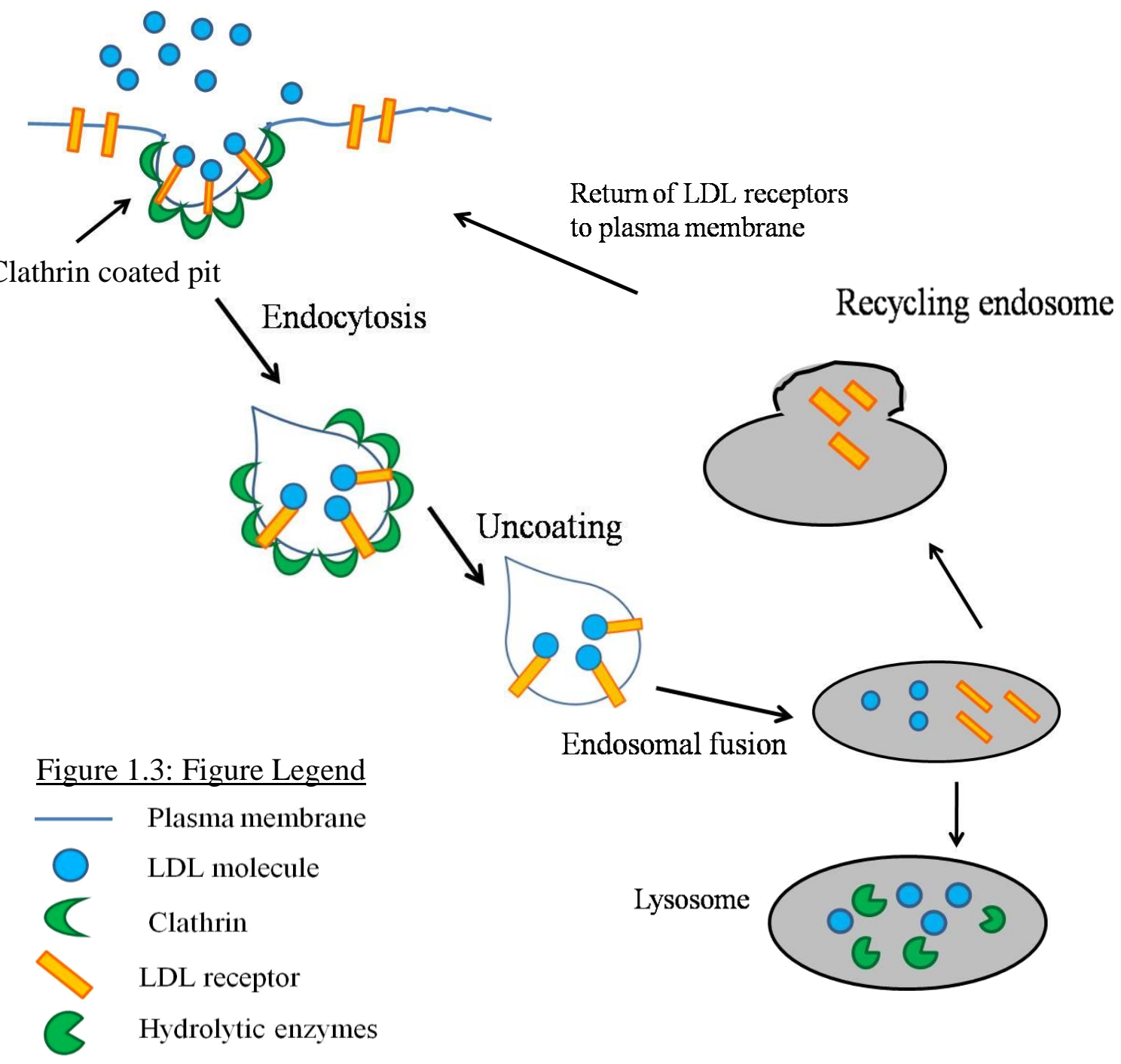

\section{Figure 1.3 Macrophage receptor mediated endocytosis}

Receptor mediated endocytosis of Low density lipoprotein (LDL) via clathrin coated pits, where the endosome fuses with the lysosome to degrade LDL in the presence of hydrolytic enzymes. LDL receptors are recycled to the plasma membrane via recycling endosomes.

This figure was adapted from Molecular biology of the cell, 2007. Albert and Johnson. 


\subsubsection{Immunomodulatory effects of anti-mitotic agents}

As mentioned above, secretion of cytokines and phagocytosis both require functional cytoskeletal machinery and membrane trafficking processes. Microtubules are involved in the production and release of cytokines such as IL-1 $\beta$ and TNF- $\alpha$ (Tsavaris et al., 2002). Additionally, the requirement of microtubules in bacterial uptake and antigen processing has long been established (von Figura et al., 1978; Harrison et al., 2002; Peachman et al., 2004; Murray et al., 2005). Anti-mitotic agents could potentially impede the above mentioned processes, leading to major implications in cancer therapy. MTA, which include both MSD and MDD, could potentially affect phagocytosis or endocytosis as well as exocytosis or cytokine secretion, as these processes are microtubule dependent (Peachman et al., 2004).

There are multiple studies investigating the effects of MSD on endocytosis as well as cytokine production. In murine macrophages, treatment with paclitaxel stabilizes microtubules and inhibits the uptake of liposomal antigen, a result also seen in colchicinetreated murine BMDM (Peachman et al., 2004). Docetaxel causes anti-proliferative effects in lymphocytes and promotes activation-induced apoptosis ( $\mathrm{Si}$ et al., 2003). In a study involving breast cancer patients, paclitaxel and docetaxel caused a pronounced effect on enhancing interferon- $\gamma$ (IFN- $\gamma$ ), IL-2, IL-6 and GM-CSF (granulocyte macrophage - colony stimulating factor), while reducing IL-1 and TNF- $\alpha$ levels (Tsavaris et al., 2002). In the same study, when compared to paclitaxel, docetaxel displayed a greater efficiency in modifying cytokine levels. The cytokines IFN- $\gamma$, IL-2 and GM-CSF, which were elevated are pre-dominantly T-helper-1 (Th1) cytokines, which activate macrophages and promote cell-mediated responses (Opal et al., 2000). They are also involved in expansion of cytotoxic T lymphocytes (CTL) in autoimmune diseases (Dinarello, 2007). An interesting 
proposition is that due to their cytotoxic effects on tumor cells, the two taxanes may induce production of cytokines via secondary recognition of tumor-derived antigens by tumorinfiltrating monocytes and B-cells (Tsavaris et al., 2002). However, these immunomodulatory events are currently poorly defined and require in-depth studies. A study in our lab also showed peloruside A treated macrophages produce decreased levels of TNF- $\alpha$, IL-12p40 and nitric oxide (NO) (Crume et al., 2007).

In addition to anti-cancer activity, taxanes are also currently being investigated as autoimmune therapeutics. Paclitaxel has been effective in inhibiting induction of experimental allergic encephalomyelitis (EAE), which is a murine model of multiple sclerosis (Cao et al., 2000). Paclitaxel has also been effective in other autoimmune diseases such as systemic lupus erythematosus (SLE) and collagen-induced arthritis (CIA) (Brahn et al., 1994; Song et al., 1998). Peloruside A and paclitaxel treatment also lead to a reduced incidence and significant delay in the development of EAE (Crume et al., 2009). This data combined with continuing studies, suggests that in addition to being valuable cancer chemotherapeutics, microtubule actives have a significant potential as immunomodulatory agents in autoimmune and inflammatory diseases.

\subsubsection{Tumor Necrosis Factor- $\alpha$}

TNF- $\alpha$ is an important cytokine, with roles in inflammation, immunity and induction of apoptosis (Scheringa et al., 1990; Tracey et al., 1994; van Horssen et al., 2006). TNF- $\alpha$ is one of the earliest, most potent cytokines secreted by macrophages in response to LPS stimulation (Carswell et al., 1975; Ding et al., 1990b; Scheringa et al., 1990). Studies have found TNF- $\alpha$ in culture as early as 2 hours and while there is conflicting evidence as to how long TNF- $\alpha$ can be detected in prolonged cultures, these differences can be explained by 
differing assay protocols (Vassalli, 1992; Tracey et al., 1993; Crume et al., 2007). TNF- $\alpha$ secretion occurs via recycling endosomes, where it is transported from the golgi to the cell membrane at the phagocytic cup for secretion (Murray et al., 2005; Manderson et al., 2007). Blocking of this recycling membrane prevents TNF- $\alpha$ secretion, and causes accumulation of TNF- $\alpha$ in the Golgi.

TNF- $\alpha$ acts via TNF receptor 1 (TNFR-1) and TNFR-2, both of which are found on almost all nucleated cells. Although TNF- $\alpha$ affinity for TNFR-2 is 5 times higher than that for TNFR-1, majority of the activity occurs via TNFR-1 (Mocellin et al., 2005; van Horssen et al., 2006). The main difference between the two receptors is the presence of the death domain (DD) on TNFR-1 and its absence on TNFR-2 (Mocellin et al., 2005; van Horssen et al., 2006). TNFR-1 is a dual receptor, with roles in the induction of apoptosis as well as in transduction of survival signals. These signaling pathways occur via the activation of transcription factors such as nuclear factor- $\kappa \mathrm{B}(\mathrm{NF} \kappa \mathrm{B})$ and the mitogenactivated protein kinase (MAPK)/cJun N-terminal kinase (JNK) (Dempsey et al., 2003; Mocellin et al., 2005; van Horssen et al., 2006). TNFR-2 lacks a death domain and is involved in immune signaling (Aggarwal, 2003; van Horssen et al., 2006). The binding of

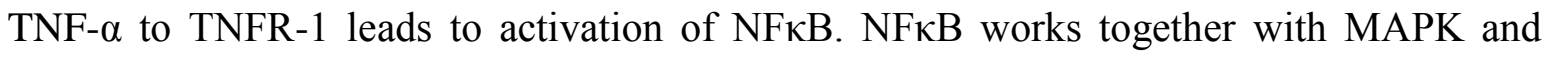
cJNK to induce transcription of anti-apoptotic, proliferative, and inflammatory genes (Vandenabeele et al., 1995; Mocellin et al., 2005; van Horssen et al., 2006). The murine TNF- $\alpha$ promoter and the expression of TNF- $\alpha$ inducing genes are partially controlled by NFאB (Baeuerle et al., 1994). Additionally, as mentioned, TNF- $\alpha$ is a potent activator of NFkB. Together this suggests that TNF- $\alpha$ once produced, is capable of stimulating its own secretion. 


\subsubsection{Nitric Oxide}

Nitric oxide (NO) results from ${ }_{\mathrm{L}}$-arginine metabolism and plays a major role in vascular and immune systems (Knowles et al., 1994; Bruckdorfer, 2005). NO is synthesized by three isoforms of nitric oxide synthase (NOS), one of which is inducible NOS (iNOS or NOS2) that induces large amounts of NO particularly from macrophagemonocyte cell lineages (Forstermann et al., 1994; Karpuzoglu et al., 2006). Production of inducible $\mathrm{NO}$ is notable in that it is produced only upon activation of macrophages/monocytes, in response to IFN- $\gamma$, LPS or TNF- $\alpha$ (Ding et al., 1988; Geller et al., 1993; Bogdan et al., 2000). iNOS also has an ability to induce higher levels of NO than those induced by the other two isoforms (Karpuzoglu et al., 2006).

Nitric oxide is a major mediator of macrophage tumoricidal activity (Stuehr et al., 1989). While macrophages produce elevated levels of NO in inflammatory disorders and microbial infections as well as in autoimmune processes, this production occurs at a later timepoint as compared to TNF-a production (Bogdan et al., 2000; Feng et al., 2002). NO is also involved in cytokine regulation, for instance, IFN- $\gamma$ stimulus produces high levels of $\mathrm{NO}$ that in turn down-regulates IFN- $\gamma$ to prevent dangerous levels of pro-inflammatory IFN- $\gamma$ production (Karpuzoglu et al., 2006). NO also regulates IL-12 production in RAW264.7 macrophages (Rothe et al., 1996). Treatment of tumor bearing hosts (TBH) with paclitaxel leads to NO production, which regulates paclitaxel-mediated IL-12 production and restores immunocompetence in these hosts (Mullins et al., 1999). This interesting finding shows that MSD can alter cytokine production, which in turn can affect macrophage immune responses. 


\subsubsection{Interferon- $\gamma($ IFN- $\gamma)$}

IFN- $\gamma$ is among the most important cytokines that is involved in activating macrophages for anti-microbial and -tumour responses, as well as causing an up-regulation of antigen-processing and presentation (Young et al., 1995; Schroder et al., 2004). Interferons are classified as type I or II depending on receptor specificity and sequence homology (Schroder et al., 2004; Platanias, 2005). There are several type I IFNs, which bind to a common type I IFN receptor, but there is only one type II IFN, IFN- $\gamma$, which binds to a distinct type II IFN receptor (Bach et al., 1997; Platanias, 2005) Macrophages secrete IL-12, IL-18 and chemokines such as macrophage inflammatory protein (MIP), the combined stimulus of which acts on T-cells and natural killer cells, leading to increased production of IFN- $\gamma$ (Munder et al., 1998; Schroder et al., 2004).

Traditional views suggested that pre-treatment with IFN- $\gamma$ activated macrophages to respond to tumour cells or TLR-agonists such as LPS; however recent studies indicate that irrespective of the time-course, or sequence of IFN- $\gamma$ stimulation and TLR-agonist challenge, macrophages are still able to respond to LPS and promote the production of NO and IL-12 (Lorsbach et al., 1993; Ma et al., 1996; Schroder et al., 2006). The production of IL-12 and NO irrespective of the sequence of IFN- $\gamma$ priming and LPS stimuli suggests synergy between IFN- $\gamma$ and LPS signalling pathways. Since macrophages still mediate priming if an IFN- $\gamma$ stimulus is presented after LPS or TLR stimulation, it is likely that signals distinct from the early TLR activation signals are involved in synergy of the two pathways (Schroder et al., 2006). Mechanisms such as activation of the transcription factors STAT-1 in the IFN pathway and NF- $\mathrm{B}$ in the TLR/LPS pathway, and crosstalk between the two signalling pathways, may be responsible for an amplification of the priming effect of IFN- $\gamma$ (Schroder et al., 2006). The ability of IFN- $\gamma$ to synergise with other cytokines and 
activate macrophages widen the possible activities that IFN- $\gamma$ has over macrophage activation (Munder et al., 1998; Schroder et al., 2004; Schroder et al., 2006)

Stimulation of macrophages with IFN- $\gamma$ results in the up-regulation of FcR $\gamma$, complement proteins that aid in opsonization of pathogens, and complement receptors such as Mac-1 that enable responses to microbial and intracellular pathogens (Schroder et al., 2004). In the presence of LPS, macrophages increase secretion of NO, IL-12 and chemokines, as mentioned above. These mediators act on NK cells to increase production of IFN- $\gamma$. IL-12 along with IFN- $\gamma$ then coordinates the link between pathogen recognition by the innate immune system, and induction of specific adaptive immune responses by promoting Th1 responses (Schroder et al., 2004). During the course of the experiments carried out in this thesis, within various assays, macrophages were primed with $100 \mathrm{U} / \mathrm{ml}$ IFN- $\gamma$ to investigate the effects of priming or absence of priming on phagocytic responses in the presence of MSDs.

\subsubsection{Shared actions of LPS and Paclitaxel on TNF- $\alpha$ production}

While paclitaxel has no structural similarity to LPS, it induces normal murine macrophage responses similar to those generated by LPS, including increased production of TNF- $\alpha$, NO, and IL-1 $\beta$ (Ding et al., 1990b; Kirikae et al., 1996). Murine macrophages also respond to LPS and paclitaxel by reducing cell surface expression of TNFR and increasing production of TNF- $\alpha$ (Ding et al., 1989; Ding et al., 1990b). The LPS mimetic activities of paclitaxel also include activation of NF- $\kappa \mathrm{B}$, tyrosine phosphorylation of MAPK and induction of LPS-inducible gene expression (Manthey et al., 1992; Ding et al., 1993; Perera et al., 1996; Byrd-Leifer C.A. et al., 2001). Interestingly paclitaxel's ability to produce inflammatory mediators and mimic LPS is observed only in macrophages from LPS 
responsive mice, while macrophages from LPS-hyporesponsive mice are incapable of producing these mediators in response to LPS or paclitaxel (Ding et al., 1990b; Bogdan et al., 1992; Kirikae et al., 1996), suggesting that LPS and paclitaxel share a common signaling pathway (Ding et al., 1990b; Burkhart et al., 1994). Additionally, the LPS mimetic property of paclitaxel is species specific and paclitaxel does not induce LPS specific responses in human cells, including human macrophages (Kawasaki et al., 2000; Kawasaki et al., 2001a; Kawasaki et al., 2001b). TLR-4 confers responsiveness to LPS; however presence of TLR-4 alone is insufficient in conferring LPS responsiveness in human cells (Kawasaki et al., 2001a). MD-2 an accessory protein, associates with TLR-4 on the cell surface and can confer LPS responsiveness in a normally unresponsive murine cell line (Kawasaki et al., 2000; Kawasaki et al., 2001a). The presence of TLR-4/MD-2 complex mediates the LPS mimicry signal by paclitaxel (Kawasaki et al., 2000). Furthermore murine MD-2 is responsible for the species specificity of paclitaxel's LPS mimicry, as demonstrated using combinations of human TLR-4, human MD-2, murine TLR-4 and murine MD-2 (Kawasaki et al., 2001a; Zimmer et al., 2008). Recent studies by Kawasaki, K. et al., 2001. further demonstrated that a glutamine $22\left(\mathrm{Gln}^{22}\right)$ point mutation in the murine MD-2 molecule led to a significant decrease in paclitaxel signaling, while LPS signaling was unaffected, suggesting that $\operatorname{Gln}^{22}$ was essential for paclitaxel but not LPS signaling (Kawasaki et al., 2001b). The mimetic activity of paclitaxel is not observed in other MSD, including peloruside A or docetaxel (Manthey et al., 1993; Crume et al., 2007). 


\subsection{Summary of known immunological effects}

Taxanes exert multiple effects on the immune system as discussed. They act as anticancer agents by directly inhibiting cellular division through microtubule stabilization. However, taxanes can also stimulate antitumor activity indirectly, by activating macrophages and cytokine production (Chan et al., 2000). Further to this, taxanes also exhibit immunosuppressive activity and may be valuable as therapeutics in autoimmune diseases (Fitzpatrick et al., 2003; Javeed et al., 2009). As mentioned above, peloruside A does not mimic LPS effects. Recent studies indicate that in limiting LPS conditions, both paclitaxel and peloruside A are capable of inhibiting LPS-induced TNF- $\alpha$ production (Crume et al., 2007). As mentioned, microtubules are involved in almost all cellular functions and alteration of the microtubule network due to MSD treatment could have farreaching effects with regards to immune responses. It is essential to understand the pathways and interactions within immune responses to bacterial infections, particularly due to the above-mentioned interactions of paclitaxel with LPS, TNF- $\alpha$ and NFkB. Furthermore, cytokine production could be impaired due to the ability of MSD to alter cytokine profiles (Chan et al., 2000). Paclitaxel, docetaxel and peloruside could affect macrophage function in different methods in vitro and in vivo. Understanding how MSD alter macrophage function will provide insights into the primary effects of MSDs during anti-cancer therapies.

\subsection{Research Aims and Hypothesis}

Based on the above information and the current knowledge of the immunomodulatory effects of MSD, we had three main research aims. We wanted to study the effects of MSD on macrophage endocytic processes, as there is limited knowledge regarding the effects of 
MSD on non-proliferating cells such as the macrophage. The first aim we had was to investigate the effect of MSD on the macrophage's ability to maintain receptor mediated endocytosis. We hypothesized that paclitaxel would not cause major alterations to this process as endocytosis is, for the most part independent of microtubules. The second aim we had was to study the effects of MSD on macrophage processes of bacterial phagocytosis and killing ability. We assumed that if there were alterations to the phagocytic process, they would mainly involve inhibition of phagocytosis, rather than killing ability being affected. This was based on the rationale that phagocytosis requires functional microtubules. Our third aim was closely related to the second aim, as we were interested in investigating the effects of MSD on cytokine production. In particular we asked if MSD affected cytokine production, which in turn would affect macrophage ability to phagocytose and kill bacteria. We also investigated the possibility of IFN- $\gamma$ activation in macrophages altering the effects of MSD. 


\section{ChAPTER 2: MATERIALS AND METHODS}

\subsection{Reagents}

Paclitaxel, purified from Taxus yannanensis, colchicine and LPS (from Escherichia coli) were purchased from Sigma-Aldrich Chemical Company (St. Louis, MO, USA). Docetaxel was purchased from LC Laboratories (Woburn, MA, USA). Ixabepilone (Ixempra) was purchased from Bristol Myers Squibb (Noble Park, Victoria, Australia). Zampanolide and mycothiazole were isolated and purified from a Tongan marine sponge Cacospongia mycofijiensis. Peloruside A was isolated and purified from New Zealand marine sponge Mycale hentscheli, and latrunculin A was isolated and purified from the Red Sea sponge Latrunculia magnifica. All sponge material was generously provided by Dr Peter Northcote (School of Chemical and Physical Sciences, Victoria University of Wellington). All drugs were dissolved in absolute ethanol, with the exception of mycothiazole, which was dissolved in dimethyl sulfoxide (DMSO). All drugs were stored at $-80^{\circ} \mathrm{C}$, with the exception of latrunculin $\mathrm{A}$ which was stored at $-20^{\circ} \mathrm{C}$ and colchicine which was stored at room temperature, protected from light. Stock concentrations were: paclitaxel at $2 \mathrm{mM}$, peloruside A, docetaxel, zampanolide and ixabepilone at $1 \mathrm{mM}$, and colchicine and latrunculin $\mathrm{A}$ at $10 \mathrm{mM}$.

Low density lipoprotein (LDL) and acetylated low density lipoprotein (ac-LDL), both from human plasma, and complexed to DiI (1,1'-dioctadecyl-3,3,3',3'-tetramethylindocarbocyanineperchlorate), were purchased from Invitrogen, Molecular Probes (Eugene, Oregon, USA). LDL-DiI and ac-LDL-DiI were stored at $4^{\circ} \mathrm{C}$, protected from light. pHrodo $^{\mathrm{TM}}$ E.coli bioparticles ${ }^{\circledR}$ for phagcytosis and Cell Tracker Orange (CMTMR) (Invitrogen, Molecular Probes) were purchased and stored at $-20^{\circ} \mathrm{C}$ protected from light. If 
re-suspension was carried out, pHrodo bioparticles were stored as $50 \mu \mathrm{l}$ aliquots to avoid multiple freeze-thaw cycles.

\subsection{Cell culture}

\subsubsection{Macrophage cell lines}

All cell culture work was carried out in a sterile class II biological safety fume hood. The murine RAW264.7 and J774.2 macrophage-like cell lines were generously provided by The Malaghan Institue of Medical Research (MIMR). Cells lines were maintained at $37^{\circ} \mathrm{C}$, 5\% $\mathrm{CO}_{2}$ atmosphere, in complete T-cell medium (CTCM; See Appendix A (Gibco, Invitrogen, Grand Island, NY, USA)). Cell lines were passaged twice weekly in T-75 tissue culture flasks (BD Biosciences, Franklin Lakes, NJ, USA) according to ATCC passage protocol (Hsueh et al., 2008) by scraping of adherent cells with cells scrapers (Greiner Bioone, Frickenhausen, Germany) and diluting to a obtain a final cell count of $1 \times 10^{6} / \mathrm{ml}$ in complete media. For experimental use, cells were freshly thawed and passaged to two passages. Following this initial passage, cells were used between 2 to 10 passages to prevent activation (Berghaus et al., 2009). Frozen cell stocks were maintained in liquid nitrogen at $1 \times 10^{6} / \mathrm{ml}$ in freezing medium (See Appendix A)

\subsubsection{The HL-60 cell line}

The human promyelocytic leukemic blood leukocyte cell line, HL-60 was generously donated by Dr John H. Miller (School of Biological Sciences, Victoria University of Wellington). HL-60 cells were maintained in RPMI-1640 medium (Roswell Park Memorial Institute, RPMI; Gibco, Life Technologies) supplemented with 10\% FCS, $100 \mathrm{U} / \mathrm{ml}$ penicillin (Invitrogen) and $100 \mu \mathrm{g} / \mathrm{ml}$ streptomycin (Invitrogen). Hl-60 cells were 
maintained at $37^{\circ} \mathrm{C}, 5 \% \mathrm{CO}_{2}$ atmosphere and passaged once weekly in T-25 tissue culture flasks (BD Biosciences, Franklin Lakes, NJ, USA) by diluting cells in a 1:10 ratio in RPMI-1640 media. Cells were used for 25-30 passages.

\subsubsection{The Escherichia coli DH5a strain}

Two Escherichia coli, (E.coli) DH5a lab strains; pOT11 GFP-labelled E.coli and PMMB207 control strain E.coli without GFP label was generously provided by Dr Ronan O’Toole (School of Biological Sciences, Victoria University of Wellington). Bacterial strains were stored in $80 \%$ glycerol at $-80^{\circ} \mathrm{C}$ and used within $4-5$ freeze/thaw cycles. E.coli was grown in a $37^{\circ} \mathrm{C}$ shaking incubator, in Luria Bertani media (LB media; See Appendix A (BD Biosciences, Franklin Lakes, NJ, USA). The GFP-label was isopropyl $\beta$-D-1thiogalactopyranoside (IPTG; Bioline, Randolph, MA, USA) dependent and not constitutively expressed. When GFP label expression was required, bacterial cultures were set up with IPTG at $100 \mu \mathrm{g} / \mathrm{ml}$ added into growth media. E.coli culture was carried out in the presence of $34 \mu \mathrm{g} / \mathrm{ml}$ chloramphenicol antibiotic (Duchefa Biochemie, Bioline, Randolph, MA, USA).

\subsection{Receptor-mediated Endocytosis (RME)}

This assay was set up to investigate the endocytosis of DiI-labeled LDL and ac-LDL (Invitrogen, Molecular Probes) by RAW264.7 cells. The basic methodology followed for this assay was as per described in (Cao et al., 2001; Yao et al., 2009). Briefly, RAW264.7 cells at a concentration of $2 \times 10^{6} / \mathrm{ml}$ in CTCM were seeded on the day of the experiment at $50 \mu \mathrm{L} /$ well in 96-well round bottom plates (Falcon). Unless otherwise stated, the cells were pre-treated for 2 hours with drug or equivalent concentration of ethanol as vehicle control 
or left untreated with equal volume of media as controls. Final concentrations and drugs are stated in figures. At the end of the drug pre-treatment period, $20 \mu \mathrm{g} / \mathrm{ml}$ of LDL-DiI or acLDL-DiI was added to the cells and a further incubation for 2 hours was carried out. Plates were removed onto ice and adherent cell populations were detached by thorough pipetting. Cells were washed once with 1x Phosphate Buffered Saline (PBS; See Appendix A (Merck, Darmstadt, Germany)) and then stained for F4/80 and CD11b markers in FACS buffer (See Appendix A; Appendix B for sources of all antibodies) and subjected to flow cytometry. Live gates were set using forward and side scatter parameters. Background fluorescence was set using isotype controls and single antibody stained cells were used to set compensations. Uptake of LDL or ac-LDL by RAW264.7 macrophages was measured by increases in DiI fluorescence intensity in the FL-2 channel. Data presented is mean from two experiments each with duplicate wells \pm SEM. Data is representative of three individual experiments.

\subsection{Phagocytosis assay - GFP-labelled E.coli phagocytosis}

\subsubsection{Seeding of RAW 264.7 or J774.2 cells}

On the day previous to the experiment, RAW264.7 or J774.2 cells at a concentration of $2 \times 10^{6} / \mathrm{ml}$ were serum-starved and seeded at $50 \mu \mathrm{L} /$ well in DMEM supplemented with 5\% FCS (experimental media) in 96 well flat bottom plates (BD Biosciences, Falcon, Franklin Lakes, NJ, USA). If cell staining with Cell Tracker Orange (CMTMR) was required, it was carried out before cell seeding. Staining was carried out as previously described (Hermans et al., 2004). Briefly, cells were washed once in CTCM and incubated at $2 \times 10^{7} / \mathrm{ml}$ in $\mathrm{CTCM}$ at $37^{\circ} \mathrm{C}$ for 15 minutes with $10 \mu \mathrm{M}$ CMTMR. This was followed by incubation in fresh CTCM for 15 minutes at $37^{\circ} \mathrm{C}$. Cells were then washed twice with 
Wash buffer (See Appendix A) before plating at the required concentration. If cell stimulation was required for the experiment (explained in the relevant sections and stated in figure legends), cells were either stimulated with 100 units/ml (U/ml) IFN- $\gamma$ overnight or left unstimulated with equal volume of experimental media added. On the day of the experiment, media was carefully replaced to prevent detachment of cells. Cells were pretreated for 45 minutes at $37^{\circ} \mathrm{C}$ with drug, ethanol (as vehicle control) or left untreated with equal volumes of media added in. Final drug concentrations and drugs used are stated in figures.

\subsubsection{Preparation of $E . c o l i$}

E.coli cultures were set up on the day previous to the experiment in LB media supplemented with $34 \mu \mathrm{g} / \mathrm{ml}$ chloramphenicol and $100 \mu \mathrm{g} / \mathrm{ml}$ IPTG as required for GFP induction. Following previously described protocols, along with some optimization adjustments as described below, on the day of the experiment, $20 \mathrm{ml}$ of overnight cultured bacteria were spun down in a $50 \mathrm{ml}$ polystyrene tube (BD Biosciences, Falcon) at 1850xg (3102 rpm) for 15 minutes (Sukumaran et al., 2003; Laroux et al., 2005). Bacteria were washed thrice in $1 \times \mathrm{PBS}$ and bacterial opsonization was carried out with BALB/c mouse serum at $10 \mu \mathrm{l} / \mathrm{ml}$ for 15 minutes at $37^{\circ} \mathrm{C}$. The optical density (OD) of the bacterial solution was measured and using $\mathrm{OD}_{600}$ of $1=1.92 \times 10^{9} \mathrm{CFU} / \mathrm{ml}$, bacteria were suspended in experimental media at required concentrations.

\subsubsection{Macrophage infection with E.coli}

At the end of the drug pre-treatment, bacteria were added to the cells at a multiplicity of infection (MOI) of 25:1 bacteria: macrophage. Concentration of drugs was maintained 
after addition of bacteria. Plates were incubated at $37^{\circ} \mathrm{C}$ for 1 hour to allow phagocytosis to occur, following which cells were placed on ice and transferred to FACS buffer. At this stage, $50 \mu 1$ of sample was removed for cytospin analysis (see section 2.8 for explanation on cytospin techniques) as required, to confirm bacterial internalization. As noted in figures, cells were stained for CD11b and F4/80 markers or left unstained in FACS buffer, followed by data collection on the flow cytometer.

\subsection{Phagocytosis assay - pHrodo phagocytosis}

\subsubsection{Preparation and seeding of RAW264.7cells}

On the day previous to the experiment, cells were washed in $0.1 \%$ FCS/PBS. Approximately $2-3 \mathrm{~mL}$ of cells at $2 \times 10^{7} / \mathrm{ml}$ was incubated with $625 \mathrm{nM} 5,6-$ carboxyfluorescein succinimidyl ester (CFSE, Sigma) for 8 minutes at room temperature. CFSE labelling was quenched with $100 \%$ FCS, at a volume equivalent to that of the initial cell volume (i.e. $2-3 \mathrm{~mL}$ as relevant) for 2-4 minutes at room temperature. This was followed by a 10 minute incubation at $37^{\circ} \mathrm{C}$, to enable excess intracellular $\mathrm{CFSE}$ dye efflux. Cells were washed thrice in $2 \%$ FCS/PBS and resuspended to a concentration of $2 \times 10^{6} / \mathrm{ml}$. The cells were then seeded in 96 well flat bottom plates at $50 \mu \mathrm{L} /$ well. If required, (explained in the relevant sections and stated in figure legends), $100 \mathrm{U} / \mathrm{ml} \mathrm{INF- \gamma}$ overnight treatment was also carried out. On the experimental day, media was carefully replaced and cells were pre-treated for 45 minutes at $37^{\circ} \mathrm{C}$ with drug, ethanol (as vehicle control) or left untreated with equal volumes of media added in. Final drug concentrations and drugs used are stated in figures. 


\subsubsection{Preparation of pHrodo E.coli bioparticles}

pHrodo bioparticles are particles labelled with a novel flurogenic dye that increases in fluorescence as the $\mathrm{pH}$ of its surroundings becomes more acidic (Molecular Probes, Invitrogen). These particles are excellent for use in phagocytic assays as phagocytosis is measured based on acidification of the particles as they change from being non-fluorescent to exhibiting a bright red fluorescence as they are taken up. According to the manufacturer's recommendations, pHrodo E.coli bioparticles were resuspended to $1 \mathrm{mg} / \mathrm{ml}$ in Uptake buffer (Hank's Buffered Saline Solution (HBSS; Sigma Chemicals) buffered with 20 mM HEPES (Gibco, Life Technologies), adjusted to $\mathrm{pH} 7.4$ with $\mathrm{NaOH}$ ). The particles were briefly vortexed, transferred to a sterile glass tube and sonicated at room temperature in a water-bath sonicator, (Soniclean 80T, Thebarton, SA, Australia) at power 8 for 5 minutes or until particles were homogenized (as visualized by the dark pink pellet at the bottom of the tube being resuspended to a uniform pink solution).

\subsubsection{Macrophage infection with pHrodo E.coli bioparticles}

At the end of the drug treatment, pHrodo particles were added to the cells at a final concentration of $0.5 \mathrm{mg} / \mathrm{ml}$. Concentration of drugs was maintained after addition of pHrodo. To enable optimal phagocytosis, plates were spun at 500xg for 3 minutes to allow contact between adhered cells and particles and incubated for 2 hours at $37^{\circ} \mathrm{C}$ in a $\mathrm{CO}_{2}$-free atmosphere. Incubation was carried out in a $\mathrm{CO}_{2}$ free environment, based on manufacturer's instructions, as elevated levels of $\mathrm{CO}_{2}$ causes artificial acidification of the Uptake buffer, leading to elevated background fluorescence. Following 2 hour infection, plates were placed on ice and supernatant was collected and stored at $-20^{\circ} \mathrm{C}$ for cytokine analysis via ELISA. The samples were then resuspend in 1xPBS was and subjected to flow cytometry 
collection. Live gates were set using forward and side scatter parameters. Background fluorescence and compensations were set using single samples of pHrodo alone and CFSE stained cells alone. Uptake of pHrodo was measured by an increase in pHrodo fluorescence intensity in the FL-2 channel. Unless otherwise described, data is presented as mean \pm SEM from two duplicate wells, and representative of one experiment.

\subsection{Gentamicin survival E.coli phagocytosis assay}

\subsubsection{Seeding of RAW 264.7 or J774.2 cells}

Cells were left unstained and seeded exactly as described in section 2.4.1. IFN- $\gamma$ stimulation if required, and drug and ethanol vehicle controls were also carried out exactly as described in section 2.4.1 Presence or absence of IFN- $\gamma$, final drug concentrations and drugs used are stated in figures.

\subsubsection{Preparation of $E . c o l i$}

Bacteria were prepared as described in section 2.4.2, with the exception that GFP labelling was not induced. If required, bacterial opsonization was carried out as described in section 2.4.2. The optical density (OD) of the bacterial solution was measured and using $\mathrm{OD}_{600}$ of $1=1.92 \times 10^{9} \mathrm{CFU} / \mathrm{ml}$, bacteria were suspended in experimental media at required concentrations.

\subsubsection{Macrophage infection with E.coli}

At the end of the drug pre-treatment, bacteria were added to the cells at a multiplicity of infection (MOI) of 25:1 bacteria: macrophage. Concentration of drugs was maintained after addition of bacteria. Plates were spun at 500xg for 3 minutes to allow contact between adhered cells and bacteria and then incubated at $37^{\circ} \mathrm{C}$ for 1 hour to allow phagocytosis. 
Following 1 hour invasion, cells were placed on ice and supernatant was collected and stored at $-20^{\circ} \mathrm{C}$ for cytokine analysis via ELISA. At this stage, $50 \mu$ l of sample was removed for cytospin analysis as required, to confirm bacterial internalization.

Following removal of supernatant, working quickly, samples that were controls for total bacterial counts or cells alone were resuspended by thorough pipetting in $100 \mu \mathrm{LB}$ broth and removed for plating on LB agar plates. The remaining samples were washed in 1xPBS and resuspended in experimental media with $100 \mu \mathrm{g} / \mathrm{ml}$ gentamicin (Gibco, Invitrogen, Grand Island, NY, USA) for 2 hours at $37^{\circ} \mathrm{C}$ in to allow killing of extracellular bacteria. Supernatant was collected again and cells were washed with 1xPBS, followed with lysis of cells by $1 \%$ TritonX-100 (Sigma Chemicals) for 5 minutes at room temperature. Cells were then pelleted by spinning at 1017xg (2300 rpm) for 5 minutes and resuspended in $100 \mu \mathrm{L} \mathrm{LB}$ broth. All samples were cultured overnight at $37^{\circ} \mathrm{C}$ on $\mathrm{LB}$ agar plates and E.coli colonies were counted the following day. Figures show bacterial survival as $\mathrm{CFU} / \mathrm{ml}$ adjusted for dilutions while plating on LB agar plates. Unless otherwise described, data is presented as mean \pm SEM of two dilutions each from two duplicate wells and is representative of one experiment.

\subsection{General flow cytometry}

Staining for RME and GFP-E.coli assays were carried out in FACS buffer (See Appendix A) in 96-well round bottom plates. Cells were stained with F4/80-fitc and CD11b-bio, with a secondary stain Streptavidin-cyc (SA-cyc). IgG-fitc and IgG-bio/SA-cyc isotype controls were included in staining. An Fc receptor $(\mathrm{FcR})$ blocking antibody, 24G2, was also used in the control mix to prevent non-specific Fc receptor ligation. Samples were incubated with antibody mix or control mix at room temperature for 10 minutes, followed 
by $1 \mathrm{x}$ wash in FACS buffer and resuspension in FACS buffer for flow cytometry. When secondary staining was done, samples were washed to remove the primary stain and incubated with the secondary stain for 10 minutes at room temperature.

Samples were collected using a BD FACSScan analytical flow cytometer (Becton Dickinson, Franklin Lakes, NJ, USA), and analyzed using BD Cell Quest Pro Software (Becton Dickinson, Franklin Lakes, NJ, USA). Samples from RME assays were collected using F4/80-FITC in the FL-1, CD11b-bio/SA-cyc in the FL-3 and LDL-DiI or ac-LDL-DiI in the FL-2 photomultiplier channels. For GFP-E.coli phagocytosis assays, samples were collected using CMTMR in the FL-1 and GFP in the FL-2 photomultiplier channels. While for pHrodo E.coli bioparticle phagocytosis, samples were collected using CFDA-SE in the FL-1 and pHrodo-SE in the FL-2 photomultiplier channels. Unless mean fluorescence levels for unloaded control cells was negligible, it was subtracted from the mean fluorescence levels of each assay condition. With experiments, background fluorescence was set using isotype controls and compensation was set using single stained cells or bacteria. All data, including dot plots and histogram generation was analyzed using Cell Quest Pro Software and graphed using GraphpadPrism version 4.0.

\subsection{Cytospin and light microscopy}

Presence of bacteria within the macrophages during infection assays were confirmed by carrying out cytospins. Cytospins are a type of centrifugation where cells are spun onto glass slides. Drying and staining of these slides then renders them suitable for microscopic analysis. After infection with bacteria, as mentioned in the sections above, $50 \mu \mathrm{L}$ of select samples were removed for confirmation of infection. Glass slides were pre-labelled with sample number and dates, and then loaded into the metal cytospin holders along with 
cardboard filters attached to cuvettes. $50 \mu \mathrm{L}$ of the samples were added to each cuvette loaded into the Cytospin 3 (ThermoShandon, Cheshire, WAT, UK). Samples were run at $1250 \mathrm{rpm}$ for 5 minutes. On completion of spin, the cuvette and the filter were carefully detached, ensuring no disarrangement to the fresh cytospin. Slides were air-dried overnight, followed by methanol fixation, and staining in 100\% Giemsa's stain (Gurr Microscopy Materials, BDH Chemicals LTD, Poole, England). (Staining protocol: See Appendix A). Slides were then viewed under a light microscope using 100x oil immersion objective.

\subsection{ELISA: Enzyme linked Immunosorbant assay}

The presence of cytokines TNF- $\alpha$, and IL-12 was detected using Enzyme-linked Immunosorbant Assay (ELISA) run according to manufacturer's instructions. All antibodies and reagents were from BD Bioscience. ELISAs were set up using cytokine specific Rat-anti-mouse TNF- $\alpha$ or IL-12 capture antibodies in 96 well ELISA plates (BD Bioscience). The capture antibody was diluted to $2 \mu \mathrm{g} / \mathrm{ml}$ in sodium phosphate buffer at $\mathrm{pH}$ 6.0 for $\mathrm{TNF}-\alpha$ and $1 \mu \mathrm{g} / \mathrm{ml}$ in sodium phosphate buffer at $\mathrm{pH} 9.0$ for IL-12. Plates were blocked with $100 \mu \mathrm{L}$ of $5 \%$ FCS in 1xPBS for 2 hours at room temperature, followed by 4 washes in $0.05 \%$ Tween in $1 \times$ PBS. TNF- $\alpha$ or IL-12 standards at $4 \mathrm{ng} / \mathrm{ml}$ (serial diluted for 12 dilutions) and samples (either neat or diluted in 5\% FCS/1xPBS) were loaded into the plates for 2 hours at room temperature. Plates were washed 4 more times, and detected with cytokine specific biotinylated rat anti-mouse-TNFa or IL-12 for 1 hour at room temperature, followed by 6 more washes, after which plates were incubated with Streptavadin-Horse-Radish-Peroxidase (SA-HRP) for 1 hour at room temperature. Plates were washed another 8 times, followed with addition of $100 \mu 1$ TMB Substrate Reagents A \& B (BD Biosciences) mixed in equal volumes. After sufficient color development, the 
reaction was quenched with $100 \mu \mathrm{l}$ Stop Buffer $\left(0.18 \mathrm{M} \mathrm{H}_{2} \mathrm{SO}_{4}, \mathrm{pH}\right.$ 6.0). Plates were read on a Versamax microplate reader (Molecular devices, Sunnyvale, CA, USA) at $450 \mathrm{~nm}$ within 10 minutes of quenching and cytokine levels were quantified against the standards using Softmax Pro Version 3.0, Molecular Devices Corporation.

\subsection{Greiss Reaction}

The Greiss reaction is normally used to measure nitric oxide production. Measuring NO production is difficult due to its production normally being in nanomolar ranges and its instability in the presence of oxygen (Archer, 1993). NO production can be indirectly measured via measuring production of its stable forms, nitrite $\left(\mathrm{NO}_{2}^{-}\right)$and nitrate $(\mathrm{Schmidt}$, 1995). A reliable, simple way of measuring NO is via the Greiss reaction as described previously (Sun et al., 2003; Crume et al., 2007). Fifty $\mu$ l of culture supernatants from pHrodo or bacterial phagocytosis assays were added to 96-well flat bottom ELISA plates. Sodium nitrite $\left(\mathrm{NaNO}_{2}\right)$ standards with a concentration range of $500-0 \mu \mathrm{M}$, and media blanks were also included for each plate. Greiss A and B reagents were then combined in equal volumes and $50 \mu \mathrm{l}$ was added to each well. Plates were read at $570 \mathrm{~nm}$ on a Versamax microplate reader (Molecular devices, Sunnyvale, CA, USA) and NO production was quantified using the standard curve.

\subsection{Metabolism and proliferation assays (MTT Assays)}

3-(4,5-dimethylthiazoyl-2-yl)-2,5-diphenyltetrazolium bromide (MTT, Sigma) is a tetrazolium salt that is reduced to purple formazan crystals in the presence of reducing agents such as NADH and NADPH (Berridge et al., 1996). The MTT assays were used to obtain dose response curves to establish the cytotoxicity of drugs to the RAW264.7, J774.2 
and HL-60 cells following 72 hour incubations. Similarly, the cytotoxic effect of paclitaxel on RAW264.7 cells and the metabolic activity of lipopolysaccharide (LPS) and paclitaxel stimulated RAW264.7 cells were tested following 2 and 8 hour incubations. Each of the MTT experiments consisted of three replicate wells, and the $\mathrm{IC}_{50}$ values $\pm \mathrm{SEM}$ in table 3.1 are based on three independent experiments, unless otherwise stated. Data is presented as percentage of control using the formula:

$$
\text { (absorbance - blank / average control - blank) x } 100 .
$$

\subsubsection{MTT assay to determine cytotoxicity of drugs}

The MTT assay was used to determine minimum inhibitory concentrations (MIC) and cytotoxicity of drugs over 72 hours in RAW264.7, J774.2 and HL-60 cells. Final concentrations and drugs are stated in figures. When setting up 72 hour MTT assays in 96 well flat bottom plates, drug stocks were made up to twice the final required concentration, followed by serial or half-log dilutions. Cells were seeded at $2 \times 10^{5} / \mathrm{ml}$ into the same wells, resulting in required concentration of drug. A cell-free media blank and a drug-free positive control were also set up on the same plate. Plates were incubated for 72 hours at $37^{\circ} \mathrm{C}, 5 \%$ $\mathrm{CO}_{2}$, at the end of which $20 \mu \mathrm{L}$ of sterile filtered $5 \mathrm{mg} / \mathrm{ml}$ MTT (Sigma) in 1xPBS was added to each well, including cell blank and control wells, to allow crystal formation. The reaction was stopped with $10 \% \mathrm{w} / \mathrm{v}$ sodium dodecyl sulphate (SDS; Sigma) in $0.01 \mathrm{~N} \mathrm{HCl}$ (MTT solubilizer solution) and the plates were incubated overnight at $37^{\circ} \mathrm{C}$ to allow dissolving of all crystals. Plates were read on a Versamax microplate reader (Molecular Devices, Sunnyvale, CA, USA) at 570nm and data was analyzed using the software Sigma Plot (Sigma Plot, Version 8.02 for Windows, SPSS Inc. Chicago, IL, USA). 


\subsubsection{MTT assays to determine changes in metabolic activity or cytokine} production due to LPS and drug treatment

The MTT assay was also used to determine changes in metabolic activity of RAW264.7 cells treated with LPS and paclitaxel. Final concentrations and drugs used are stated in figures. These MTT assays were run on culture experiments that were previously set up as follows. Cells were stimulated with $5 \mathrm{ng} / \mathrm{ml}$ (high) or $0.3 \mathrm{ng} / \mathrm{ml}$ (low) doses of LPS and simultaneously either treated with a range of concentrations of paclitaxel or left untreated. Following a 2 or 8 hour incubation, the supernatant was removed, either leaving $100 \mu \mathrm{L}$ in all wells, or replacing wells with CTCM to a final volume of $100 \mu \mathrm{L}$. The supernatants collected were stored at $-20^{\circ} \mathrm{C}$ for TNF- $\alpha$ analysis. As described above, $20 \mu \mathrm{L}$ of sterile filtered $5 \mathrm{mg} / \mathrm{ml} \mathrm{MTT}$ was added then to each well; plates were incubated at $37^{\circ} \mathrm{C}$ for 2 hours, followed by addition of MTT solubilizer solution to stop the reaction. Plates were incubated overnight at $37^{\circ} \mathrm{C}$ to allow dissolving of all crystals, and were read on a Versamax microplate reader (Molecular Devices) at 570nm and data was analyzed using the Sigma Plot software.

\subsection{Confocal Microscopy}

\subsubsection{Cell staining and preparation}

RAW264.7 cells were plated onto $35 \mathrm{~mm}$ glass bottomed confocal dishes (MatTek, Ashland, MA) in $1 \mathrm{ml}$ of CTCM 2 days prior to the experiment and allowed to grow to $80 \%$ confluency (as visually determined). On the day of the experiment, cells were gently washed with fresh CTCM and $600 \mu \mathrm{l}$ Uptake buffer (see section 2.5.2) was used as experimental media. 
If cells were being stained as noted in figure legends, staining was carried out on the adherent cells on the day of the experiment. Cells were stained with CFSE as previously described in section 2.5.1. One change was increasing the number of wash steps as cells were adherent and dye efflux was harder to achieve. Cells were also stained using the blue fluorescent, cell permeant nucleic acid stain Hoechst 33342 component of the Image-it ${ }^{\mathrm{TM}}$ kit (Invitrogen, Molecular Probes). For Hoechst staining, cells were washed once in Krebs buffer (See Appendix A) followed by incubation with $1 \mu \mathrm{M}$ Hoechst dye for 10 minutes at room temperature. Cells were then washed 5-6 times in Krebs buffer and $2-3$ times in Uptake buffer after which they were incubated for experiments in $600 \mu l$ Uptake buffer. When staining cells with CFSE and Hoechst, CFSE staining was carried out before Hoechst staining.

\subsubsection{Fluorescent microscopy and Image acquisition}

Following a 45 minute drug or ethanol vehicle control treatment, pHrodo particles were added to the dishes at a final concentration of $25 \mu \mathrm{g} / \mathrm{ml}$. Plates were incubated at $37^{\circ} \mathrm{C}$ in a $\mathrm{CO}_{2}$ free incubator, for 30 minutes following pHrodo addition before data collection. Cells were left untreated or were treated with $1 \mu \mathrm{M}$ paclitaxel, $1 \mu \mathrm{M}$ latrunculin $\mathrm{A}, 10 \mu \mathrm{M}$ colchicine or equivalent concentration of ethanol as vehicle control. Drug concentration was maintained following addition of pHrodo. Cells were observed using an Olympus FluoView FV1000 confocal laser scanning biological microscope (inverted model IX81) under 60x oil immersion objective lens. Images were taken every 30 minutes after pHrodo addition for 4 hours, with the exception of vehicle treated cells, where images were taken at 30, 150, 240, 330 and 420 minutes after pHrodo addition. This was to done to ensure all drug and control treatments were able to be carried out on the same day for the same time intervals. 
During imaging, dishes were enclosed in a heat plate insulated, humidified double layer chamber (INU-ZILCS-F1 model, Tokai Hit Co., LTD, Japan) at $37^{\circ} \mathrm{C}$. Images were obtained using XYZ scanning series to obtain a XY confocal image through the thickness of the sample. At each timepoint, at least 3 fields of view were randomly selected and images were acquired using lasers and standard filters as described in Table 2.1. Phase contrast images were acquired using Differential Interference Contrast (DIC) with confocal pHrodo overlay. XY confocal images were also taken using CFDA-SE, Hoechst, pHrodoSE and phase contrast along with and Hoechst, pHrodo-SE and phase contrast alone.

\subsubsection{Analysis}

Images were collected and analyzed using Olympus FV10-ASW software. There was one plate set up per drug; each plate was imaged in at least 3 randomly chosen fields of view, at every 30 minute time point. For analysis, at least 350 cells in total were manually counted from the images at each 30 minute timepoint. The pHrodo positive cells were visually identified and expressed as a percentage of the total number of cells counted in the field of view. 
Table 2.1 List of filters, lasers and excitation-emission ranges for dyes used during confocal imaging.

\begin{tabular}{|c|c|c|c|}
\cline { 2 - 4 } \multicolumn{1}{c|}{} & $\begin{array}{c}\text { Excitation }- \text { Emission } \\
\text { Wavelength }\end{array}$ & Filter & Laser \\
\hline CFDA-SE & $492-517 \mathrm{~nm}$ & 488 & 473 \\
\hline pHrodo-SE & $560^{*}-585 \mathrm{~nm}$ & 554 & 559 \\
\hline Hoechst 33342 & $350-461 \mathrm{~nm}$ & DAPI & 405 \\
\hline Phase contrast & N/A & N/A & 473 \\
\hline
\end{tabular}

Table 2.1 Range of imaging parameters for confocal imaging with 3 colour dyes and non-confocal differential phase contrast imaging. * indicating pHrodo-SE is comparably excited using the 488 argon-ion laser as specified by manufacturer. 


\subsection{Statistics}

Data from MSD cytotoxicity and MTT assays of paclitaxel/LPS interations over 2 and 8 hours was analyzed using SigmaPlot software version 8 (Systat Software Inc., Point Richmond, CA, USA) using the four parameter logistic curve to generate $\mathrm{IC}_{50}$ curves. Phagocytic assay data was analyzed with GraphpadPrism 4.0 (Graphpad Software Inc., San Diego, CA, USA), using one-way ANOVA tests with Bonferroni or Newman-Keuls post hoc test. Standard curves for ELISA and Greiss reaction data were generated by using Softmax Pro Version 3.0 (Molecular Devices Corporation, Sunnyvale, CA, USA), and the data was analyzed in GraphpadPrism using one-way ANOVA with Bonferroni post hoc test. ELISA data from paclitaxel and LPS interaction over 2 and 8 hours were analyzed using two-way ANOVA with Bonferroni post hoc test in GraphpadPrism. Confocal experiments were analyzed using Olympus FV10-ASW software and two-way ANOVA with Bonferroni post hoc test in GraphpadPrism. 
Chapter 3: Cytotoxic effects of MSD

on RAW264.7 and J774.2 macrophages

\section{CHAPTER 3: CYTOTOXIC EFFECTS OF MSD ON RAW264.7 AND J774.2 MURINE}

\section{MACROPHAGES.}

\subsection{Introduction}

Previous studies involving MSD have focussed on their anti-mitotic effects in proliferating cells. However, because of the diverse role of microtubules in cellular function (Peachman et al., 2004), it is possible that MSD also alter microtubule mediated cellular function in non-proliferating cells. Studies in our lab looking at MSD and macrophages have focussed on primary macrophages; in particular, bone marrow derived macrophages (BMDM) (Crume et al., 2007), and the effects of MSD on these macrophages are distinct to cytotoxic effects (Crume et al., 2007; Crume et al., 2009). In this thesis, we investigate the effects of MSD on macrophages, which are non-proliferating cells. The cells used within our studies were the murine macrophage cell lines RAW264.7 and J774.2 macrophages. Using murine macrophage cell lines offer advantages of immortalized, relatively stable cells with an adherent macrophage like phenotype. Both these cell lines exhibit the macrophage maturity markers F4/80 and CD11b and have been used in numerous studies as models for macrophages (Maurya et al., 2007). RAW264.7 cells predominantly display Th1 properties, while J774.2 cells predominantly display Th2 properties (Su et al., 2001). However, there are conflicting views on the origin of RAW264.7 macrophages. The RAW264.7 cells display an inflammatory phenotype when stimulated with LPS and produce high levels of TNF- $\alpha$ and NO (Fitzgerald et al., 2000; Sosroseno et al., 2002; Berghaus et al., 2009). This is similar to responses exhibited by macrophages from C57BL/6 mice (Santos et al., 2006), however RAW264.7 were derived from a BALB/c mouse (Raschke et al., 1978). Additionally, BALB/c mice are unable to 
produce Th1 lymphocytes, and macrophages from these mice are not as sensitive to IFN- $\gamma$ and LPS stimulation as C57BL/6 macrophages (Santos et al., 2006). Another study comparing RAW264.7 macrophages and C57BL/6 BMMO found the cells to closely resemble each other (Berghaus et al., 2009).

However, both in vitro and in vivo RAW264.7 and J774.2 macrophages display phenotypes similar to those of primary macrophages. RAW264.7 macrophages are also considered similar to murine peritoneal macrophages (Ralph et al., 1977a; Sakagami et al., 2009). Both cell lines are good models for macrophage studies and although there are differences in cell signalling pathways, cell surface receptor expression and cytokines produced, for the most part these cell lines are similar to using primary macrophages (Maurya et al., 2007). In addition, both cell lines have been used extensively to study phagocytosis (Walter et al., 1980; Renwick et al., 2001; Suzuki et al., 2006; Anand et al., 2007).

The RAW264.7 and J774.2 cell lines are both proliferative, however when studied over shorter time frames, such as 2 to 4 hours, the cells are non-proliferative. Thus within the context of our assays, the cells are non-proliferative macrophages. To demonstrate this, we tested the cytotoxic effects of the MSDs on these cell lines using MTT assays. MTT assays are simple colorimetric assays that are used to measure cytotoxicity of a compound or cell viability (Kasugai et al., 1990; Pozzolini et al., 2003). MTT is a yellow, watersoluble tetrazolium salt, and metabolically active cells are able to reduce this to blue waterinsoluble formazan crystals. MTT assays work on the premise that if metabolism is unaffected, an increase in the amount of blue crystals correlates to cell proliferation. Thus if a low absorbance value is obtained after drug treatment, it would indicate that toxic effects of 
the drug lead to cellular death. MTT assays provide minimum inhibitory concentration (MIC) or $\mathrm{IC}_{50}$ values, which indicate the efficacy of the drug in the particular cell line. We tested the efficacy and cytotoxicity of paclitaxel, peloruside A and docetaxel on RAW264.7 (Fig 3.1.1) and J774.2 macrophages (Fig 3.1.2) over 72 hours. These assays were done to ensure that both cell lines were susceptible to the MSDs but also to rule out the possibility of mistaking cytotoxic effects for alterations in functionality of macrophages due to MSD treatment. We expected the cells to be sensitive to the drugs and compared the $\mathrm{IC}_{50}$ values obtained to those of HL-60 cells (Fig 3.1.3), which have been previously described (Hood et al., 2002; Sahin et al., 2008). The effects of high dose (10 $\mu \mathrm{M})$ paclitaxel on RAW264.7 cells over 2 and 8 hours were also studied to ensure there were no cytotoxic effects over the duration of the experiments (Fig 3.1.4). Further to this, since all assays were seeded at the same cell concentration, we looked at the proliferation of untreated cells within each of the MTT assays to ensure that cytotoxicity results were not due to absence of cell proliferation (Fig 3.1.5) Drugs and final concentrations are stated in the figures. We expected RAW264.7 and J774.2 macrophages to react similarly to all 3 MSD. Further, we also expected these cell lines to be sensitive to the compounds as they proliferate in-vitro. $\mathrm{IC}_{50}$ figures are representative dose response curves for the drug with its corresponding $\mathrm{IC}_{50}$ value. 


\subsection{Results}

Figure 3.1.1
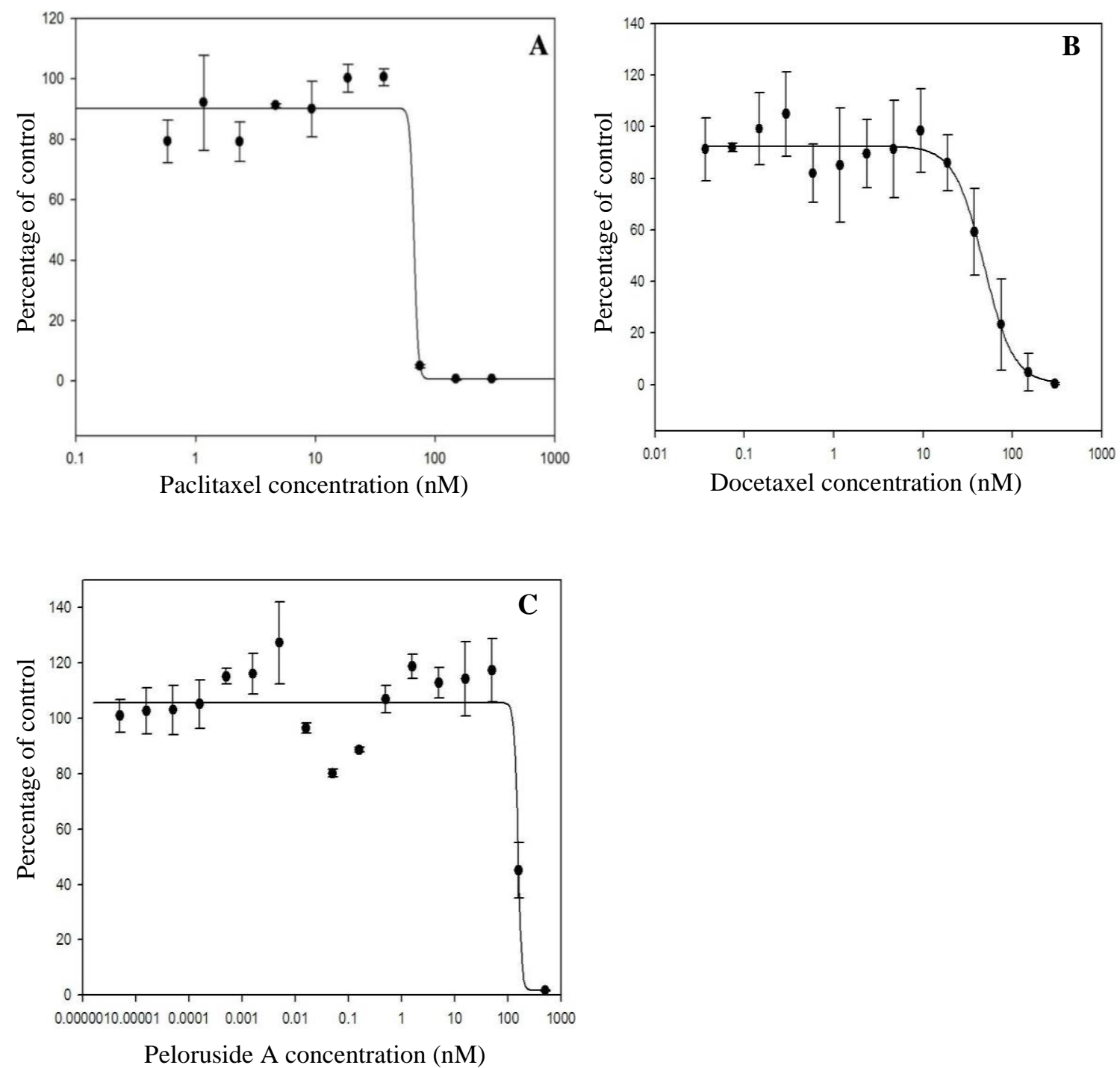

Figure 3.1.1 Representative $\mathrm{IC}_{50}$ curves for (A) paclitaxel, (B) docetaxel and (C) peloruside A in RAW264.7 macrophages. Cells were seeded at 2x10 $/ \mathrm{ml}$ for all assays and incubated with (A) paclitaxel (B) docetaxel or (C) peloruside A for 72 hours. Results are shown as a percentage of control, where controls are non-drug treated cells. Curves are a representative curve out of 3 individual experiments, except peloruside A which is out of 2 individual experiments. Data presented as mean \pm SEM. 
Figure 3.1.2
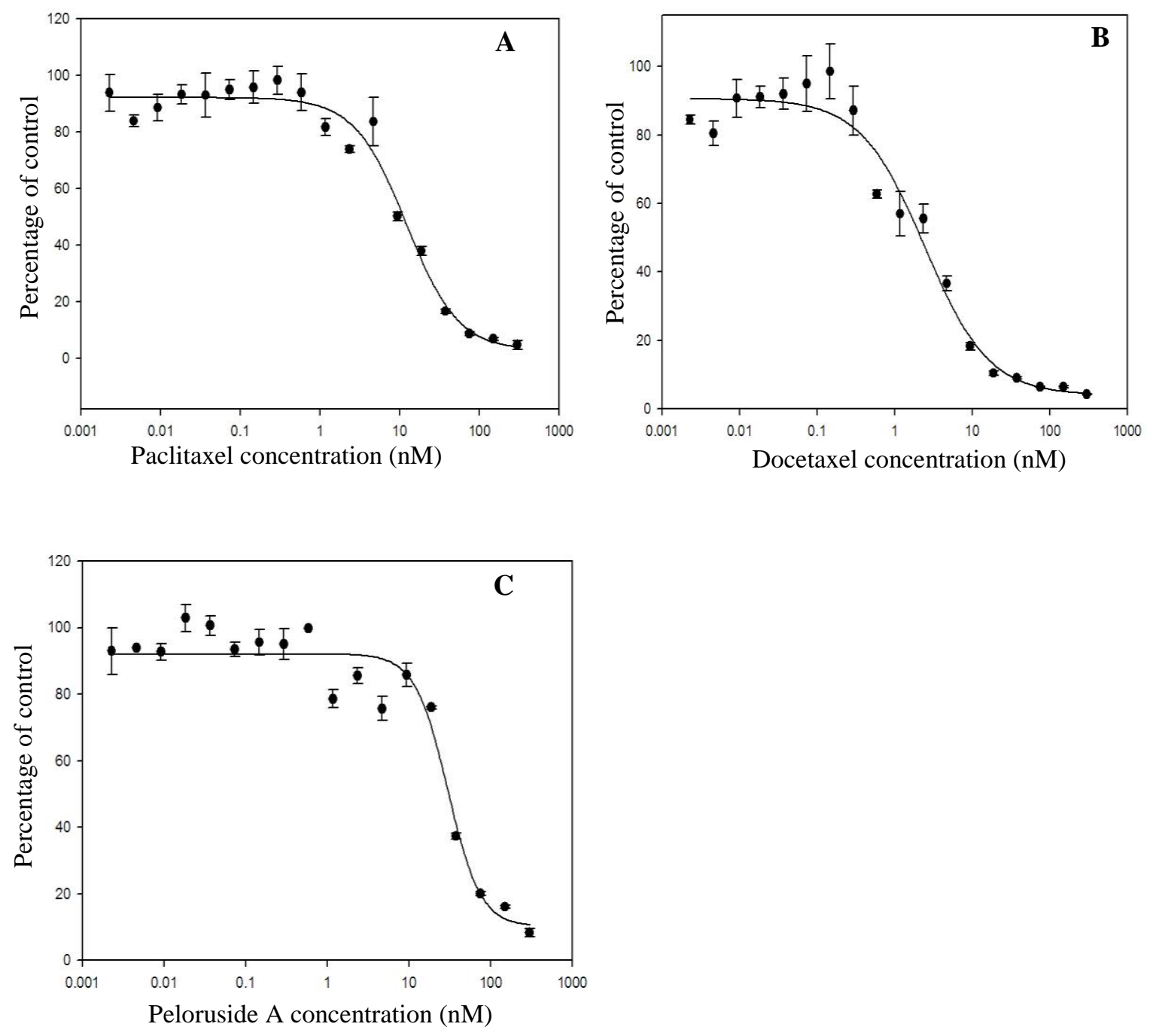

Figure 3.1.2 Representative $\mathrm{IC}_{50}$ curves for (A) paclitaxel, (B) docetaxel and (C) peloruside $\mathbf{A}$ in $\mathbf{J 7 7 4 . 2}$ macrophages. Cells were seeded at $2 \times 10^{5} / \mathrm{ml}$ for all assays and incubated with (A) paclitaxel (B) docetaxel or (C) peloruside A for 72 hours. Results are shown as a percentage of control, where controls are non-drug treated cells. Curves are a representative curve out of 3 individual experiments, except peloruside A which is out of 2 individual experiments. Data presented as mean \pm SEM. 
Figure 3.1.3
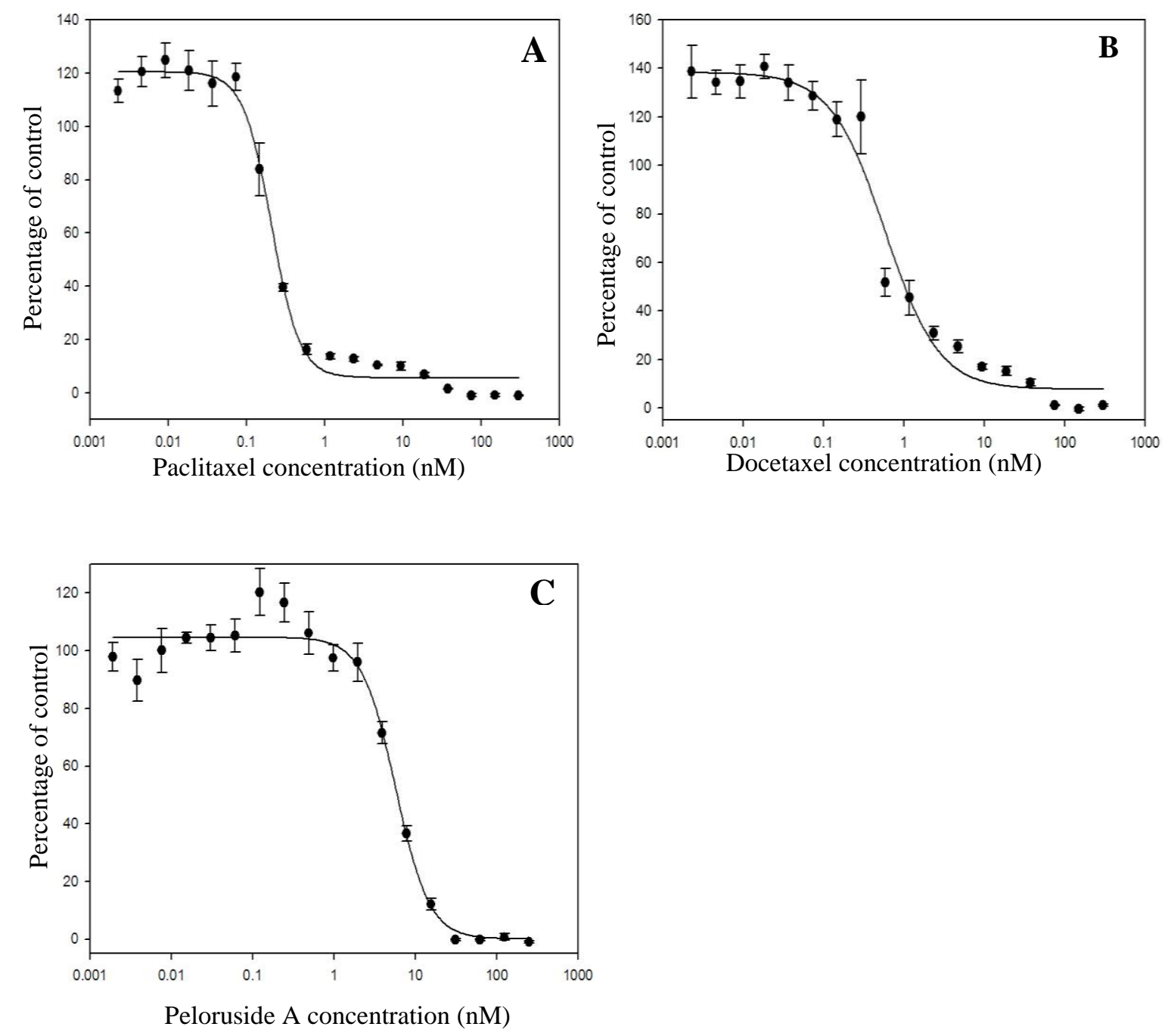

Figure 3.1.3 $\mathrm{IC}_{50}$ curves for (A) paclitaxel, (B) docetaxel and (C) peloruside $\mathrm{A}$ in HL60 cells. Cells were seeded at $2 \times 10^{5} / \mathrm{ml}$ for all assays and incubated with (A) paclitaxel (B) docetaxel or (C) peloruside A for 72 hours. Results are shown as a percentage of control, where controls are non-drug treated cells. Curves are a representative curve out of 3 individual experiments, except peloruside A which is out of 2 individual experiments. Data presented as mean \pm SEM. 
Table 3.1 IC $_{50}$ values for paclitaxel, docetaxel and peloruside A in RAW264.7, J774.2 and HL-60 cell lines.

\begin{tabular}{|c|c|c|c|c|}
\hline & & \multicolumn{3}{|c|}{ MSD } \\
\hline & & paclitaxel & docetaxel & peloruside $A$ \\
\hline \multirow{3}{*}{$\begin{array}{c}\text { Cell } \\
\text { Lines }\end{array}$} & RAW264.7 & $75.54 \mathrm{nM} \pm 8.64$ & $48.31 \mathrm{nM} \pm 3.71$ & $155.93 \pm 2.13 \mathrm{nM}$ \\
\hline & J774.2 & $19.81 \mathrm{nM} \pm 4.42$ & $2.93 \mathrm{nM} \pm 0.50$ & $11.31 \mathrm{nM} \pm 7.93$ \\
\hline & HL-60 & $1.2 \mathrm{nM} \pm 0.83$ & $0.84 \mathrm{nM} \pm 0.26$ & $6.98 \mathrm{nM} \pm 1.59$ \\
\hline
\end{tabular}

Table 3.1 $\mathrm{IC}_{50}$ values obtained from 72 hour incubations of each specified drug with three cell lines. Data presented as mean of three $\mathrm{IC}_{50}$ values $\pm \mathrm{SEM}$, except peloruside A in RAW264.7 cells which is a mean of two $\mathrm{IC}_{50}$ values. 


\section{Figure 3.1.4}

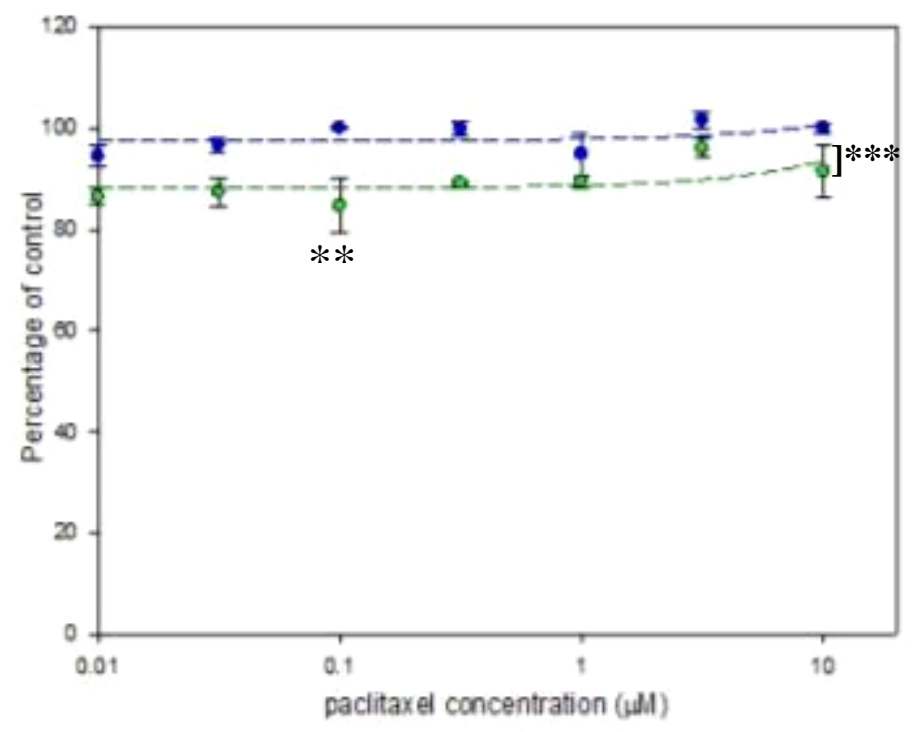

Figure 3.1.4 MTT curves for paclitaxel at 2 and 8 hours in RAW264.7 cells. Cells were seeded at $2 \times 10^{5} / \mathrm{ml}$ for both assays and incubated with paclitaxel for $2(O)$ hours or 8 (O) hours. Results are shown as a percentage of control, where controls are non-drug treated cells. Curves are presented as mean \pm SEM of two replicates. $* * p<0.018$ hours vs 2 hours at $0.1 \mu \mathrm{M}$ dose, $* * * p<0.001$ overall trend 8 hours vs 2 hours, two-way ANOVA with Bonferroni post test.

\section{Figure 3.1.5}

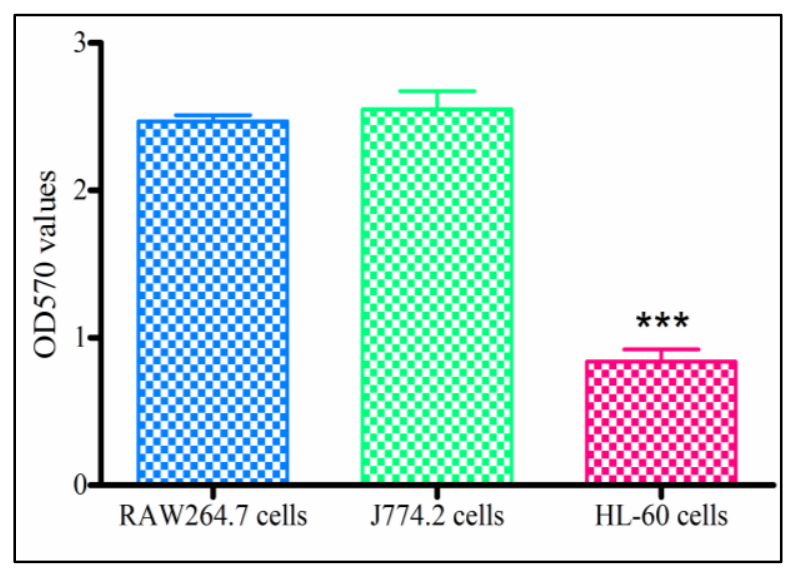

Figure 3.1.5 OD $_{570}$ values from a 72 hour MTT assay on RAW264.7, J774.2 and HL-60 cells. All the 3 cell lines were seeded at $50 \mu \mathrm{L} /$ well at an inital concentration of $2 \times 10^{5} / \mathrm{ml}$, and incubated in media alone for 72 hours. $\mathrm{OD}_{570}$ absorbance values due to MTT reduction, indicate level of cell proliferation or level of viable cells after 72 hours. RAW264.7 and J774.2 cells have proliferated to a greater extent as compared to HL-60 cells, in 72 hours. Results are presented as mean \pm SEM of absorbance values. ***p<0.001; One-way ANOVA with Bonferroni Correction, RAW264.7 vs HL-60 cells and J774.2 vs HL-60 cells. 


\subsection{Discussion}

The first set of experiments was aimed at understanding the cytotoxic effects of the 3 MSD on the proliferative macrophage cell lines. As these cell lines were proliferative, we expected the MSD to have cytotoxic effects on the cells; however we were interested in establishing the efficacy of the drugs in each cell line. Also, since we were interested in examining the effects of MSD on macrophage function, it was necessary to establish if the cell lines were resistant or susceptible to the 3 MSD. Although RAW264.7 and J774.2 are both macrophage cell lines, the effect of MSD could vary in both these cell lines. We also expected the efficacy of the 3 MSD to vary in the macrophage cell lines as compared to HL-60 cells.

We found that J774.2 cells were more susceptible to all the 3 MSD when compared to RAW264.7 cells, while HL-60 cells were the most susceptible to all the 3 drugs. Compared to J774.2 cells, paclitaxel was 4-fold more resistant, docetaxel was 17-fold more resistant, and peloruside A was 14-fold more resistant in RAW264.7 cells. There was also a much higher fold of resistance when comparing RAW264.7 to HL-60 cells for all the 3 drugs. Paclitaxel was 63-fold, docetaxel was 58-fold and peloruside A was 22-fold more resistant in RAW264.7 cells when compared to HL-60 cells. Interestingly, comparatively higher $\mathrm{IC}_{50}$ values for peloruside A was seen in all the 3 cell lines, although resistance to paclitaxel in J774.2 cells was marginally higher than resistance to peloruside A. This increase in fold resistance could be due to microtubule-mediated resistance mechanisms (discussed below). Another possibility for peloruside A being relatively more resistant in all three cell lines could be related to its tubulin binding, in particular the isoform of tubulin to which it binds, 
and the location of the binding site, which is still un-known (Jimenez-Barbero et al., 2006; Huzil et al., 2008). If peloruside A was unable to bind onto its tubulin site, cell lines would presumably exhibit resistance towards the drug, as it would be unable to exert mitotic or immune-mediated effects in these cells.

Resistance to anti-mitotic drugs often results from over-expression of membrane transporter proteins called ATP-binding cassette (ABC) transporters (Geney et al., 2002; Jordan et al., 2004). Resistance to paclitaxel is also well established and occurs via overexpression of P-glycoprotein (P-gp) (Geney et al., 2002; Risinger et al., 2008). P-gp is a small transmembrane glycoprotein encoded by a family of multi-drug resistance (MDR) genes. There are two MDR genes in humans, MDR1 and MDR2, while mice and rodents express three MDR genes, mdr1a, mdr1b and mdr2 (Gupta, 2003) While P-gp is expressed in humans and mice, it is not clear if P-gp is expressed on murine hematopoietic cells (Gupta, 2003). Furthermore, if murine macrophages expressed P-gp it does not confirm the presence of P-gp in RAW264.7 murine macrophages.

Currently there are no reports of RAW264.7 cells expressing P-gp and it would be interesting to investigate the possible presence of $\mathrm{P}$-gp or other $\mathrm{ABC}$-transporters on RAW264.7 cells, which could be responsible for increased resistance to not just paclitaxel but also docetaxel and possibly other MSDs. In addition, what makes the fold resistance seen in RAW264.7 cells towards peloruside A remarkable, is that peloruside A is not a good substrate for the P-gp drug pump (Gaitanos et al., 2004). The presence of alternative MDR pumps, with peloruside A as a substrate would be worth investigating, especially with the aim of developing peloruside $\mathrm{A}$ as a chemotherapeutic in paclitaxel resistant cancers. However, it would be more beneficial to investigate microtubule mediated 
resistance, especially due to the fact that peloruside A has an un-identified tubulin binding site, novel to that of the taxanes.

Resistance to MSD could also arise from microtubule-related mechanisms, such as expression of regulatory proteins, post-translational modifications, differences in levels of tubulin content, alterations in microtubule associated protein (MAP) content and presence of tubulin isotypes (Burkhart et al., 2001; Orr et al., 2003; Jordan et al., 2004). Microtubules have multiple $\alpha$ - and $\beta$-tubulin isotypes that are encoded by different genes, located on different chromosomes. While the structure of tubulin is conserved, tubulin isotypes differ in their carboxy-terminal tails (the last 20-27 amino acids) (Kavallaris, 2010). Further to this, each isotype can undergo post-translational modifications, which can lead to alterations in microtubule interactions with microtubule associated proteins (MAPs) (Jordan et al., 2004; Kavallaris, 2010). The difference in tubulin isotypes and its differing expression in tissues and cell lines contribute towards explaining the development of resistance to various MSD in different cell lines.

Two point mutations in $\beta$-tubulin near the taxane binding site, $\beta 274^{\text {Thr } \rightarrow \text { Ile }}$ and $\beta 282^{\mathrm{Arg} \rightarrow \mathrm{Gln}}$ have resulted in resistance to epothilones and taxanes via impaired tubulin binding (Giannakakou et al., 2000). Studies have shown that increased expression of $\mathrm{M} \beta 2$, a class II $\beta$-tubulin isotype, contributed to development of high resistance to paclitaxel and altered expression of $\mathrm{M} \beta 2$ lead to paclitaxel and docetaxel resistance in J774.2 cells (Haber et al., 1995). Given the difference in fold-resistance seen with paclitaxel and docetaxel in RAW264.7 compared to J774.2 cells, it is possible that RAW264.7 macrophages express a $\beta$-tubulin isotype different to that of J774.2 macrophages. Further, the level of expression of the tubulin isotype could also differ, and this could contribute to increased resistance. If 
RAW264.7 cells had an acquired point mutation in $\beta$-tubulin near the taxane binding site, this would also lead to an increase in resistance to paclitaxel and docetaxel.

Alternate explanations for the increased fold resistance to MSD seen with the two macrophage cell lines could be related to increased microtubule dynamics. Paclitaxel and other such MSDs are capable of altering microtubule dynamics even at low concentrations (Kelling et al., 2003; Jordan et al., 2008), indicating that their effects on dynamics are more potent. Paclitaxel resistant A549-T12 and -T24 lung cancer cells were also seen to have increased microtubule dynamics, indicating that microtubule dynamics were a significant factor in MSD resistance (Goncalves et al., 2001). Many of the factors mentioned above, such as tubulin isotypes, MAP expression and alteration and tubulin mutations could probably affect microtubule dynamics. All these factors in combination could account for the higher fold-resistance seen in the RAW264.7 cells to not only paclitaxel but also docetaxel and peloruside A.

The MTT assays were also carried out to ensure that there were no cytotoxic effects of the MSD on the cells over the time-course of the experiment. Both macrophage cell lines have doubling times of between 12 to 18 hours (Rebres et al., 2004; American Type Culture Collection, 2007; Sakagami et al., 2009). While MSD are toxic to proliferating cells, within the time period of our assays we expected there to be no proliferation and thus no cytotoxic effects. Any alterations in the response of treated cells as compared to untreated cells could therefore be attributed to effects other than cytotoxic effects of MSD.

This was demonstrated by carrying out a 2 and 8 hour MTT with paclitaxel treatment on RAW264.7 cells. We used a two-way ANOVA test with Bonferroni post test to analyse 
the results. We tested if proliferation was affected by varying concentrations of paclitaxel over the 2 or 8 hour incubation time and varying incubation time alone, independent of concentrations. We found that cell viability was not affected after 2 hours of paclitaxel treatment, indicating that there was no proliferation over 2 hours. Additionally, there was no effect of varying concentration over the 2 hour incubation. At 8 hours however; analysis indicated that there was a significant decrease in cell viability after incubation with paclitaxel as compared to viability at 2 hours. Using the Bonferroni post test, comparing 2 and 8 hours, at the concentration of $0.1 \mu \mathrm{M}$, there was a decrease in cell viability. However at higher concentrations, there was no significant difference between 2 and 8 hours suggesting that the significance could be due to assay variability. The $0.1 \mu \mathrm{M}$ concentration was the only dose at which there was a significant change in cell viability. Despite this, using the Bonferroni post test to analyze the interactions between time and concentration at 2 and 8 hours, we found a highly significant difference between the two incubation times, indicating that overall there was a decrease in viability at 8 hours as compared to 2 hours.

Interestingly, there was no significant difference in treatment with increasing concentrations of paclitaxel over 8 hours. We found that the higher range of paclitaxel concentrations did not affect the viability of cells at 2 or 8 hours as compared to the lower range of concentrations. Furthermore, cells were able to survive at doses of $10 \mu \mathrm{M}$ paclitaxel for both 2 and 8 hours, although survival was lower at 8 hours. Ten $\mu \mathrm{M}$ is a high dose as compared to the $\mathrm{IC}_{50}$ value of paclitaxel in RAW264.7 cells. However, previous studies carried out in non-proliferative primary macrophages, have used these high doses to study effects of MSD (Khandani et al., 2007; Robinson, 2009). Therefore we tested the survival and viability of RAW264.7 cells at these high doses of paclitaxel over short 
periods of time to rule out cytotoxic effects of MSD on cells. Overall, the results enabled us to affirm that for the assays carried out further along the study, since the maximum incubation time in the presence of drug was 4 hours or less, any effects seen were not due to cytotoxic effects on cell viability. A reason for cytotoxic effects at 8 hours would most likely be the increased exposure to high doses of drug. It is likely that this cytotoxic effect would not be present if paclitaxel doses were lower and incubation was still carried out over 8 hours.

An interesting observation was that there was no increase in the metabolic activity of RAW264.7 cells stimulated with paclitaxel. Bone marrow derived macrophages have been seen to increase their metabolic activity when stimulated with paclitaxel (Crume et al., 2007), however this increase was after a 72 hour incubation with paclitaxel. Given the LPS mimicry of paclitaxel, this increase in metabolism over 72 hours is not surprising. Macrophages respond to LPS or paclitaxel by increasing production of inflammatory mediators (Raetz, 1990; Tracey et al., 1994), and this would lead to increase in cell metabolism. While our results show a slight increase in cell metabolism after 8 hours of paclitaxel stimulation, this is not seen at 2 hours of paclitaxel stimulation. Since paclitaxel has LPS mimicry, the increase in metabolism at 8 hours could be due to an increase in production of inflammatory mediators, or due to an increase in proliferation due to LPSlike signal (Crume et al., 2007). It is possible that increasing incubation periods with paclitaxel could lead to increased metabolism in RAW264.7 cells; however the timing of this incubation would be limited due to the proliferative nature of RAW264.7 cells, where incubation with paclitaxel over extended time periods, would lead to cell cycle arrest and cellular death. 
The MTT assays allowed us to investigate another aspect of cell proliferation. We looked at proliferation of cells in relation to presence or absence of MSDs. Since we set up controls of non-drug treated cells for each of the MTT assays, it seemed obvious to ensure that the cytotoxic effects we observed on the cell lines were not due to a lack of cell proliferation over 72 hours. We compared the 72 hour $\mathrm{OD}_{570}$ values for wells with untreated cells after addition of MTT, which indicates proliferation of cells and cell viability. Since all 3 cell lines were seeded at the same concentration, we initially expected that HL60 cells would have higher proliferation and in comparison the macrophage cell lines would have lower rates of proliferation over 72 hours. If this held true, we hypothesized that the remarkably high $\mathrm{IC}_{50}$ values, especially for paclitaxel and peloruside $\mathrm{A}$ in RAW264.7 cells could be a result of lack of proliferation over 72 hours. Surprisingly, when looking at $\mathrm{OD}_{570}$ values, we found that the RAW264.7 and J774.2 cells had similar levels of cell proliferation and in contrast the HL-60 cells had significantly lower proliferation rates.

The decreased proliferation of the HL-60 is explained by the doubling time of the cells, which is between 36 to 48 hours (Gallagher et al., 1979). As mentioned earlier, the macrophage cells have a doubling time of 12 to 18 hours. With the increased macrophage proliferation, the MSDs should have had a greater cytotoxic effect, resulting in lower IC50 values. However, the $\mathrm{IC}_{50}$ values were higher indicating that the cells were resistant despite their higher proliferation rates. Contrary to our expectations, the HL-60 cells proliferated less over 72 hours and thus the MSDs had less of a cytotoxic effect, leading to comparatively lower $\mathrm{IC}_{50}$ values as compared to the macrophage cell lines. An improvement to the HL-60 MTT assays would be to run them over 48 hours, or alter initial seeding of cells to compensate for differences in proliferation speeds. The difference in 
MTT incubation protocol for HL-60 cells could also explain the differences in the $\mathrm{IC}_{50}$ values we found as compared to the data previously published. Previous $\mathrm{IC}_{50}$ values of $7 \pm$ $4 \mathrm{nM}$ (Hood et al., 2002) published for peloruside A in HL-60 cells, have been comparable to our results of $6.98 \pm 1.59 \mathrm{nM}$. However, HL-60s were seen to have 48 hour $\mathrm{IC}_{50}$ values of $22 \pm 8 \mathrm{nM}$ for paclitaxel (Hood et al., 2002) and $20 \mathrm{nM}$ and $5 \mathrm{nM}$ for respectively for paclitaxel and docetaxel (Sahin et al., 2008). In contrast, our data gave 72 hour $\mathrm{IC}_{50}$ values of $1.2 \pm 0.83$ and $0.84 \pm 0.26 \mathrm{nM}$ for paclitaxel and docetaxel, respectively. It is likely that cell death was higher due to incubation times being 72 hours, thus resulting in lower $\mathrm{IC}_{50}$ values. However we wanted to compare MSD effects on the macrophages and the HL-60s and so maintained constant conditions for all the assays.

We investigated the cytotoxic effects of $3 \mathrm{MSD}$, namely paclitaxel, docetaxel and peloruside A, on 2 proliferating macrophage cell lines RAW264.7 and J774.2. We also compared these effects to effects on a proliferating promyelocytic blood leukaemia cell line, HL-60. Our results indicated that while all 3 proliferating cell lines were susceptible to the MSD, there was a varying degree of susceptibility with HL-60 cells being most affected, followed by J774.2 cell and lastly RAW264.7 cells. We also found that there was a 14-fold and 22-fold increase in resistance to peloruside A in RAW264.7 cells as compared to J774.2 and HL-60 cells respectively. This was noteworthy for two reasons; firstly, peloruside A is normally active in MDR cell lines (Gaitanos et al., 2004), and secondly, peloruside $\mathrm{A}$ is also known to bind to a tubulin site different from that of paclitaxel (Gaitanos et al., 2004); both of which suggest that peloruside A should be active in paclitaxel resistant cell lines. Further to this, we were able to show that over short 
incubations of 2 to 8 hours, MSD did not have cytotoxic effects on proliferating cells. Taken together, our findings suggest that the use of the macrophage cell lines was a valid choice for the endocytic studies we were interested in carrying out. It also showed that cell lines from similar lineages could exhibit vastly different responses to MSD treatment. This is an important consideration when investigating reasons for the development of MDR strains or when exploring potential new anti-cancer therapeutics. 


\section{CHAPTER 4: EFFECT OF MSD ON RECEPTOR MEDIATED ENDOCYTOSIS}

\subsection{Introduction}

Endocytosis is the process by which cells internalize small molecules and particles and target them to specific organelles within the cytoplasm (Smythe et al., 1991; Aderem et al., 1999). Endocytosis includes phagocytosis, pinocytosis, clathrin-dependent receptormediated endocytosis (receptor mediated endocytosis; RME), and clathrin-independent endocytosis (Mellman, 1996). The RME pathway was first studied using uptake of lipoprotein, and since then this mechanism has been well characterized (Goldstein et al., 1979; Gaffney et al., 1985). When ligand binding occurs, the receptor complex is internalized and fuses with an endosome. Receptor and ligand dissociate and the receptor is recycled, while the ligand-containing vesicles fuse with a lysosome (Mellman, 1996). This trafficking along the endocytic pathway mainly depends on functional microtubules and actin (Aderem et al., 1999). While trafficking of the receptor-ligand bound vesicle is more microtubule-dependent compared to the initial events of receptor mediated uptake, both processes depend on a functional, interacting cytoskeleton (Peachman et al., 2004).

Low density lipoprotein (LDL) is the major cholesterol carrier and its uptake is mediated by the LDL receptor (Brown et al., 1979b). The uptake of un-modified or native LDL is slow when compared to uptake of LDL that is modified by acetylation, or oxidation (Goldstein et al., 1979; Henriksen et al., 1981; Nagelkerke et al., 1983). Further to this, uptake of modified LDL by in vitro tissue macrophages or murine macrophages is preferential over native LDL uptake (Goldstein et al., 1979; Brown et al., 1983; Plüddemann et al., 2007). Also, the presence of various, highly efficient ac-LDL receptors results in higher levels of ac-LDL being endocytosed. 
Since the LDL and ac-LDL uptake pathways are well established and it is known that both these ligands are endocytosed via receptor mediated pathways (Sparrow et al., 1989), we utilized this pathway to test the ability of paclitaxel-treated macrophages to endocytose LDL and ac-LDL. We were interested in investigating any impairment in endocytic ability after paclitaxel treatment. Since the ligands have multiple receptors, we used both ligands for endocytosis experiments to gain a more detailed picture of the effects of paclitaxel. The two ligands were conjugated to a fluorescent probe 3,3'-dioctadecylindocarbocyanine (DiI) to enable endocytic studies to be carried out (Stephan et al., 1993). We used RAW264.7 macrophages for endocytic studies, which have previously been shown to endocytose LDL and its modified forms (Yao et al., 2009). We also characterized the macrophages by staining with the widely used macrophage markers, CD11b (MAC-1) and F4/80 (Khazen et al., 2005; Shen et al., 2008). 


\subsection{Results}

Initially, we characterized the phenotype of the RAW264.7 cells (Fig 4.1.1) by staining with fluorescently labelled antibodies F4/80-fitc and CD11b-bio/SA-cyc. Live and dead cell gates were set up based on forward and side scatter FACS plots, which characterize cells based on size and granularity. IgG-fitc and IgG-cyc antibodies were also used as a non-specific IgG control and to set isotype control gates. We also tested the uptake of varying concentrations of LDL and ac-LDL in un-treated RAW264.7 cells to determine the dose at which sufficient, measurable uptake was achieved (Fig 4.1.2 and Fig 4.1.3). Analysis of results using two-way ANOVA with Bonferroni correction, showed a significant dose dependent increase in levels of uptake. This allowed us to determine 20 $\mu \mathrm{g} / \mathrm{ml}$ of LDL and ac-LDL as the dose at which there was sufficient, measurable rates of endocytosis occurring, and any changes to uptake in the presence of MSD would be apparent. For both histograms in figure 4.1.2, a shift to the right represents increased uptake of LDL or ac-LDL. However, there was no significant difference between the levels of LDL and ac-LDL endocytosed at each concentration of the reagent. The background MFI of cells alone was low enough to be negligible and was not subtracted from the MFI of cells with LDL or ac-LDL.

Changes to the ability of macrophages to endocytose LDL or ac-LDL when pretreated with varying doses of paclitaxel was also investigated (Fig 4.1.4 and Fig 4.1.5). The endocytic ability of paclitaxel treated cells was compared to the ability of vehicle treated cells as we also wanted to ensure there were negligible effects from vehicle control on the endocytic process. Final concentration of vehicle was the same as final concentration of vehicle in the drug treated cells, and vehicle concentration was maintained throughout the assay. For both histograms in figure 4.1.4, a shift to the right represents increased uptake of 
LDL or ac-LDL. We did not expect a significant decrease in the ability of paclitaxel treated macrophages to carry out receptor mediated endocytosis. Using a two-way ANOVA with Bonferroni correction to compare vehicle and paclitaxel treated cells, showed that there was no significant enhancement or impairment in the ability of paclitaxel treated cells to endocytose LDL or ac-LDL within the assay incubation period of 4 hours. 
Figure 4.1.1

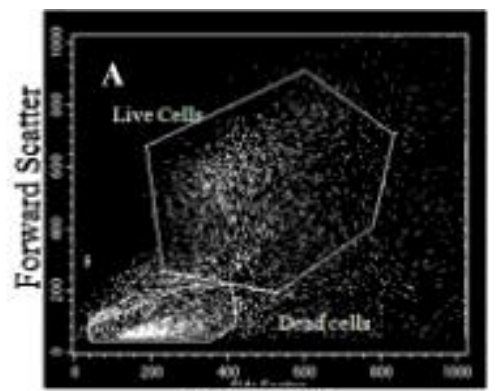

Side Scatter

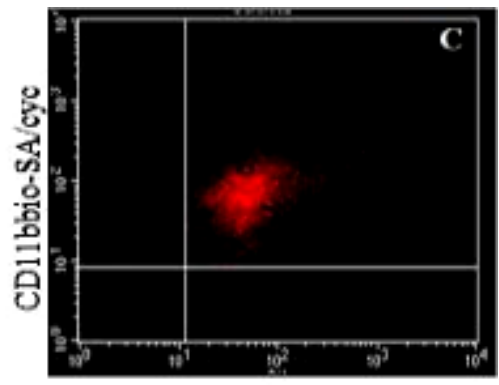

DiI-LDL.

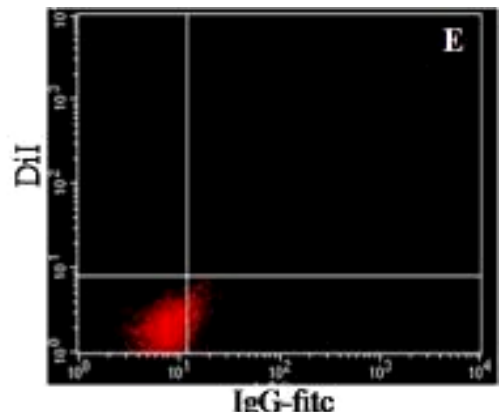

IgG-fitc

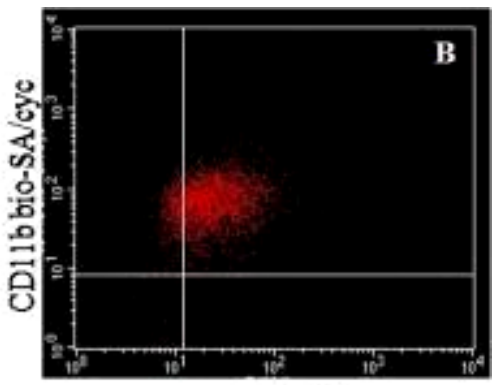

F4/80-fitc

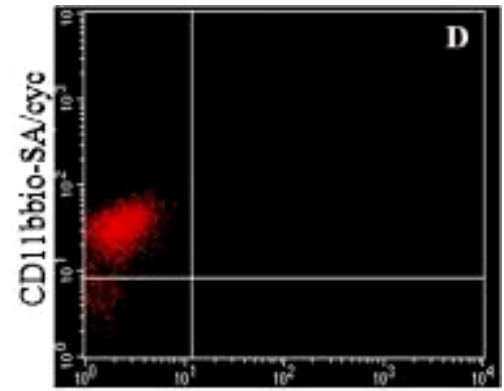

DiI-L.DL.

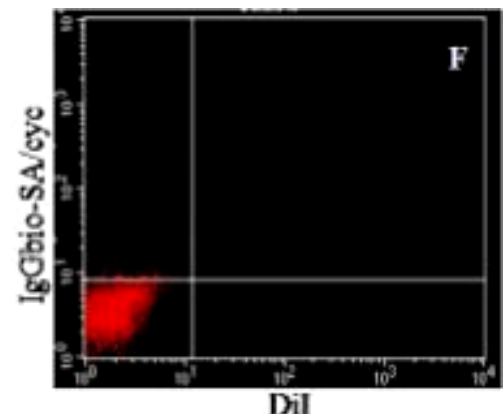

Figure 4.1.1 Flow cytometry plots showing F4/80 and CD11b characterized RAW264.7 macrophages incubated with DiI labelled LDL particles. (A) RAW264.7 macrophages showing a live and dead cell gate. (B) RAW264.7 macrophages are F480 and $\mathrm{CD} 11 \mathrm{~b}^{+}$compared to (E, F) IgG isotype controls. (C) RAW264.7 cells endocytose DiILDL and the cell population shifts compared to (D) cell population in the absence of DiILDL. 
Figure 4.1.2

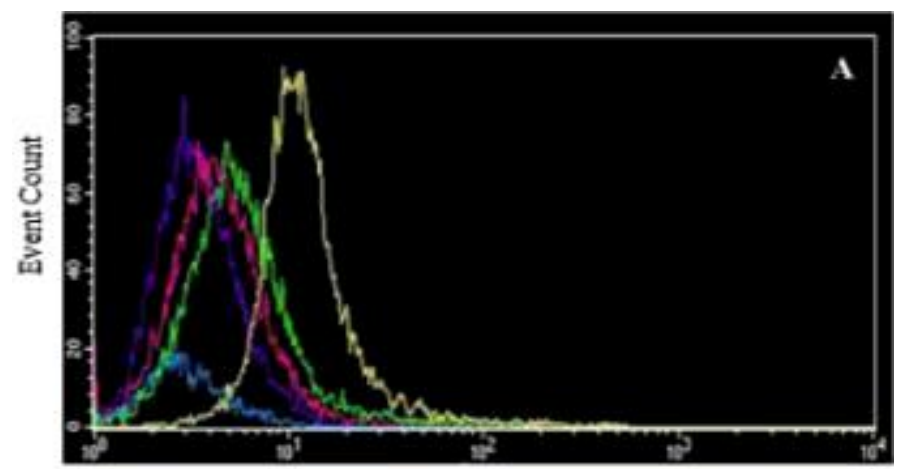

4.1.2 A. Figure Legend

- IgG antibody stained cells -

- $\mathrm{CD} 11 \mathrm{~b}+\mathrm{F} 4 / 80+$ cells with:

- $0 \mu \mathrm{g} / \mathrm{ml}$ DiI-LDL

- $5 \mu \mathrm{g} / \mathrm{ml}$ DiI-LDL

- $10 \mu \mathrm{g} / \mathrm{ml}$ DiI-LDL

- $20 \mu \mathrm{g} / \mathrm{ml}$ DiI-LDL

Dil-H.DL. uptake

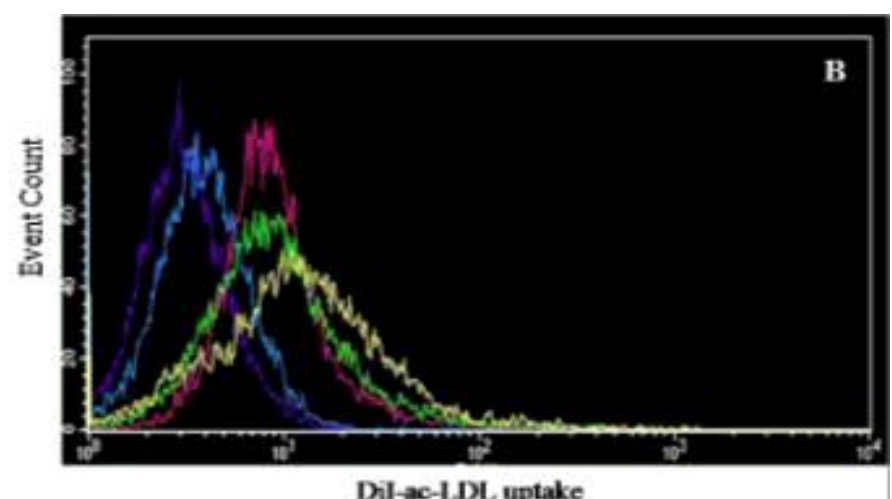

4.1.2.B Figure Legend

- IgG antibody stained cells

- $\mathrm{CD} 11 \mathrm{~b}+\mathrm{F} 4 / 80+$ cells with:

- $0 \mu \mathrm{g} / \mathrm{ml}$ DiI-ac-LDL

- $5 \mu \mathrm{g} / \mathrm{ml}$ DiI-ac-LDL

- $10 \mu \mathrm{g} / \mathrm{ml}$ DiI-ac-LDL

- $20 \mu \mathrm{g} / \mathrm{ml}$ DiI-ac-LDL

Figure 4.1.2 Dose dependent increases in receptor mediated endocytosis of DiI-LDL and DiI-ac-LDL by RAW264.7 macrophages. (A) Dose-dependent increase in endocytosis of DiI-LDL by RAW264.7 cells. Events are gated on $\mathrm{CD}_{11 \mathrm{~b}}^{+} / / \mathrm{F} 4 / 80^{+}$ macrophages. Uptake was assessed as an increase in MFI. Optimal uptake is seen with 20 $\mu \mathrm{g} / \mathrm{ml}$ of DiI-LDL. (B) Dose-dependent increase in endocytosis of DiI-ac-LDL by RAW264.7 cells. Events are gated on $\mathrm{CD} 11 \mathrm{~b}^{+} / / \mathrm{F} 4 / 80^{+}$macrophages. Uptake was assessed as an increase in MFI. Optimal uptake is seen with $20 \mu \mathrm{g} / \mathrm{ml}$ of DiI-ac-LDL. Both histograms are representative of 2 individual experiments. 
Figure 4.1.3

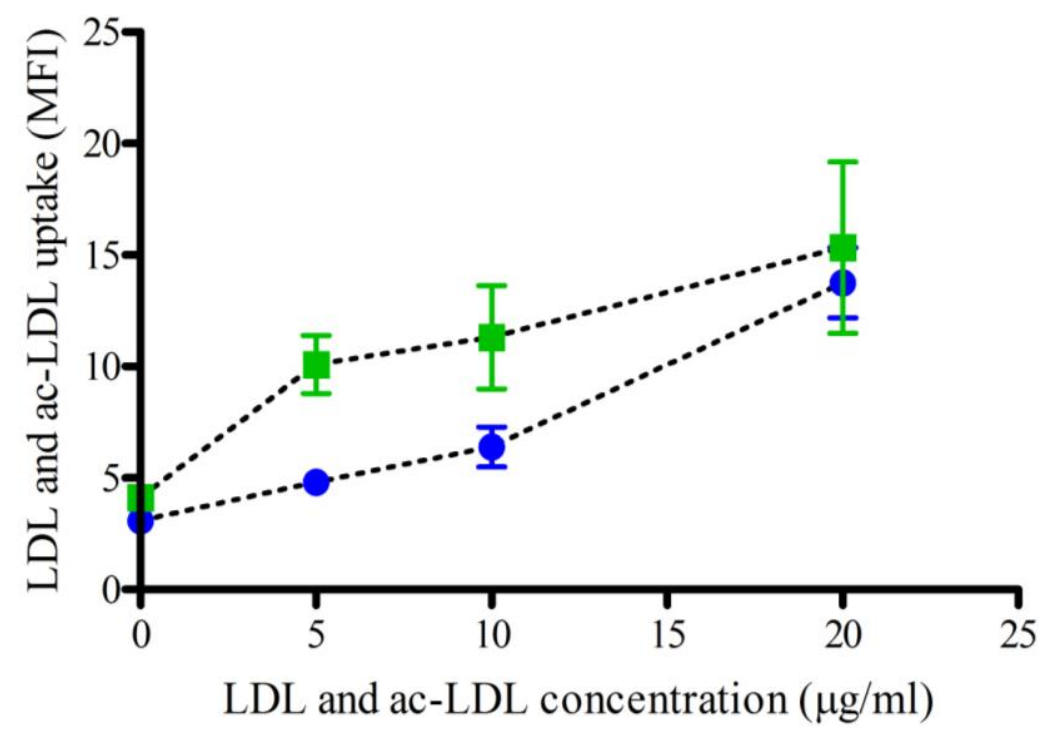

Figure 4.1.3 Uptake of LDL -- -- or ac-LDL -- --- in RAW264.7 macrophages. Cells were seeded at $2 \times 10^{6} / \mathrm{ml}$ and incubated with $0 \mu \mathrm{g} / \mathrm{ml}, 5 \mu \mathrm{g} / \mathrm{ml}, 10 \mu \mathrm{g} / \mathrm{ml}$, or $20 \mu \mathrm{g} / \mathrm{ml}$ of LDL -- -- or ac-LDL -- -- for 2 hours. There was no significant difference between the levels of LDL or ac-LDL endocytosed at each concentration, however there was a significant dose-dependent increase in the level of uptake of both compounds. Graph is representative of two experiments. Data points show mean fluorescence intensity \pm SEM from duplicate wells within one experiment. $* * * p<0.001 \mathrm{LDL}$ and ac-LDL uptake (MFI) vs. concentration of LDL and ac-LDL. Two-way ANOVA with Bonferroni correction, 
Figure 4.1.4

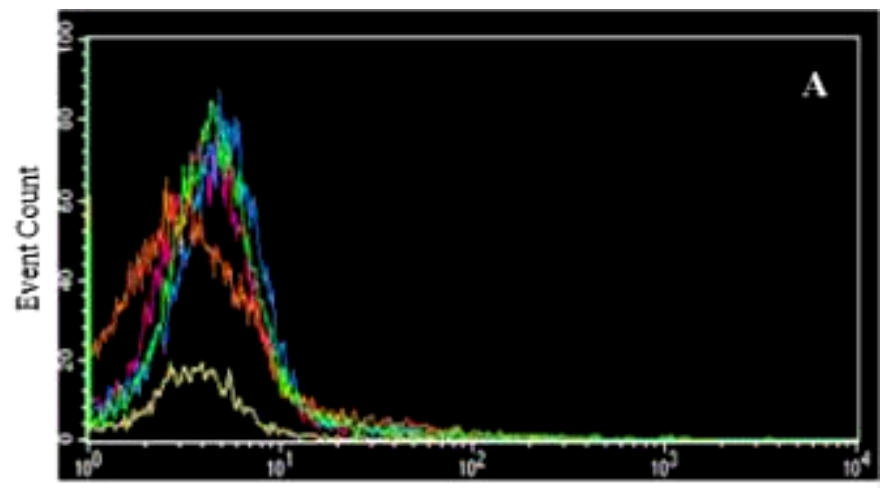

4.1.4. A Figure Legend

- Untreated cells alone

- Cells with:

- $0 \mu \mathrm{M}$ paclitaxel

- $0.1 \mu \mathrm{M}$ paclitaxel

- $1 \mu \mathrm{M}$ paclitaxel

- $10 \mu \mathrm{M}$ paclitaxel

Dil-LDL uptake

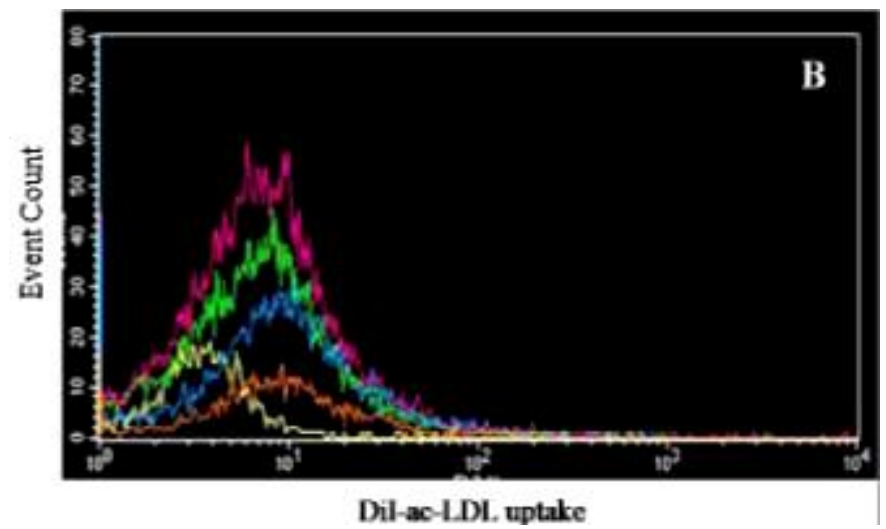

4.1.2.B Figure Legend

- Untreated cells alone

- Cells with:

- $0 \mu \mathrm{M}$ paclitaxel

- $0.1 \mu \mathrm{M}$ paclitaxel -

- $1 \mu \mathrm{M}$ paclitaxel -

- $10 \mu \mathrm{M}$ paclitaxel _-

Figure 4.1.4 Uptake of DiI-LDL and DiI-ac-LDL by RAW264.7 macrophages in the presence of paclitaxel. (A) Endocytosis of DiI-LDL by RAW264.7 cells treated with paclitaxel at varying doses. There is no shift or change in the MFI which represents uptake of DiI-LDL at the varying paclitaxel doses. Uptake was assessed as an increase in MFI. (B) Endocytosis of DiI-ac-LDL by RAW264.7 cells treated with paclitaxel at varying doses. There is no shift or change in the MFI which represents uptake of DiI-ac-LDL at the varying paclitaxel doses. Uptake was assessed as an increase in MFI. Both histograms are representative of 2 individual experiments. 
Figure 4.1.5
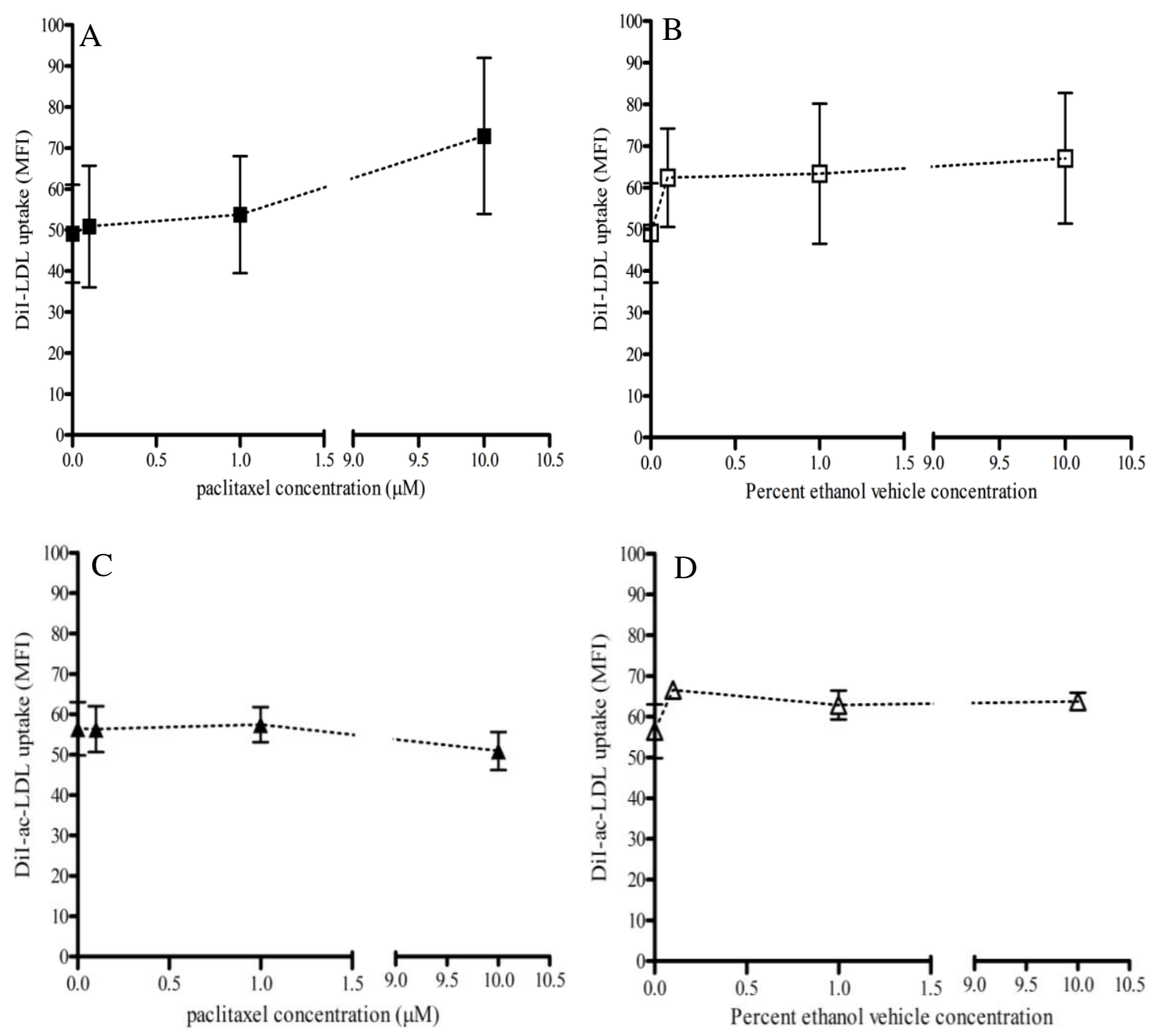

Figure 4.1.5 Uptake of DiI-LDL or DiI-ac-LDL in paclitaxel-treated RAW264.7 macrophages. Cells were seeded at $2 \times 10 \% \mathrm{ml}$ and pre-treated for 2 hours with paclitaxel at $0.1 \mu \mathrm{M}, 1 \mu \mathrm{M}$ or $10 \mu \mathrm{M}$ doses, $(\mathbf{A}, \mathbf{C})$ or an equal concentration of ethanol as a vehicle control (B, D). This was followed by incubation with $20 \mu \mathrm{g} / \mathrm{ml}$ of DiI-LDL (A, B) and (C, D) DiI-ac-LDL for two hours. (A) There was no significant difference in the ability of paclitaxel-treated macrophages -- $\square$-- to endocytose LDL as compared to ethanol vehicletreated macrophages -- $\square$--. (B) There was no significant difference in the ability of paclitaxel-treated macrophages -- $\mathbf{\Delta}$-- to endocytose ac-LDL as compared to vehicle treated macrophages -- $\Delta$--. Similar levels of LDL and ac-LDL were endocytosed. There was no enhancement or impairment of the endocytic process in the paclitaxel-treated macrophages. The ethanol vehicle controls are representative of the percentage of ethanol present in the paclitaxel drug treated samples, each data point on the vehicle control graph corresponds to the $0,0.1,1$ or $10 \mu \mathrm{M}$ dose of paclitaxel used. Graphs are representative of three individual experiments. Data points are mean fluorescence intensity from two individual experiments \pm SEM. p>0.05, two-way ANOVA with Bonferroni post test. 


\subsection{Discussion}

This section of experiments was carried out to investigate the effects of the MSD, paclitaxel on the macrophage endocytic process, in particular receptor mediated endocytosis (RME). As the name suggests, RME is uptake of small $(<0.5 \mu \mathrm{m})$ particles via a receptor and involves binding of the ligand to its receptor (Goldstein et al., 1979; Smythe et al., 1991). A lot of the initial events of receptor mediated uptake are microtubule independent. However paclitaxel stabilizes microtubules, and processes that are a part of RME such as trafficking of the vesicle containing ligand-receptor complex, endosomal fusion with the ligand, and recycling of the receptor is microtubule dependent (Oda et al., 1995). Furthermore, unlike processes such as phagocytosis, receptor mediated endocytosis does not require cell motility. With these facts in mind, we were interested in investigating any inhibition or other effects of paclitaxel on this endocytic process.

We found that RAW264.7 cells were able to endocytose LDL and ac-LDL. Levels of ac-LDL taken up were marginally but not significantly higher, as compared to LDL. We expected the difference in uptake of these two ligands to be much more distinct, due to the fact that there are more cellular receptors for ac-LDL (Goldstein et al., 1979; Gaffney et al., 1985). The lack of difference in levels of LDL versus ac-LDL taken up could possibly be due to the fact that these assays were run in complete media, which contains $10 \%$ fetal calf serum (FCS). A recent publication found that RAW264.7 cells can be activated by cytokines and growth factors in serum, which leads to their activation and subsequent enhancement of native LDL uptake (Yao et al., 2009). The publication also found that RAW264.7 cells can take up LDL and its modified forms via macropinocytosis, and not just receptor mediated endocytosis (Yao et al., 2009). This could also account for there being no difference in the level of LDL and ac-LDL endocytosed by RAW264.7 cells. 
Treatment with paclitaxel was carried out at 3 doses; $0.1 \mu \mathrm{M}, 1 \mu \mathrm{M}$ and $10 \mu \mathrm{M}$, which have been used previously (Jordan et al., 1993; Lewkowicz et al., 2008; Robinson et $a l ., 2009)$. We wanted to ensure that the doses picked would stabilize microtubules but also be similar to previously tested doses. There was no change in the level of LDL or ac-LDL taken up by RAW264.7 macrophages in the presence of paclitaxel. While we did not expect dramatic decreases in the ability of treated macrophages to endocytose LDL or ac-LDL, the level of uptake was surprisingly similar to vehicle treated controls for both ligands. This suggests that microtubule stabilization or bundling does not affect endocytosis of ligands such as LDL via their receptors. This is not surprising however since the initial endocytic processes are known to be clathrin- and actin-dependent but microtubule independent (Schroer et al., 1991; Aderem et al., 1999).

While initial endocytic events do not seem to be affected by paclitaxel, the processes following initial uptake could possibly be affected by paclitaxel or other such MSD. The ligand binds receptors that are clustered in clathrin coated pits on the plasma membrane (Anderson et al., 1982). Once ligand binds, these coated pits invaginate from the membrane to from a vesicle. This vesicle is trafficked along the microtubule pathway to fuse with the early endosome, after which fusion with the lysozome occurs, where receptors are recycled and LDL is degraded (Anderson et al., 1982; Smythe et al., 1991). Studies have found that microtubules are required for endosomal sorting and for segregation of the ligand from the receptor to facilitate dissociation (Goltz et al., 1992). Other studies have also shown that while microtubules are not required for the uptake of ligands, in the presence of microtubule inhibitors, vesicle movements are diminished and the degradation of endocytic material is hindered (Schroer et al., 1991). It was also seen that delivery of vesicle cargo 
from vesicles to late endosomes was absent in the presence of microtubule inhibitors, suggesting the requirement of microtubules for this process (Gruenberg et al., 1989). While the role of microtubules within cellular function is obvious, what is less clear is the extent to which these microtubule-dependent processes are maintained in the absence of dynamic microtubules. Our results suggest that stabilization of microtubules via MSD treatment does not affect endocytosis of ligands such as LDL or its modified forms. However, once uptake has occurred, MSD treated macrophages could possibly be unable to process these LDL laden vesicles. An interesting detail that could explain the lack of inhibition seen in paclitaxel treated macrophages during endocytosis of LDL, is that microtubule poisons frequently leave an intact pool of cytoplasmic microtubules that may be competent to support vesicle transport over short distances within the cell (Schroer et al., 1991) Another possibility could be that vesicle transport to early endosomes is achieved on paclitaxel stabilized microtubules, and only events following this are inhibited. It would be ideal to study this pathway in primary macrophages, as they can be incubated over longer time periods in the presence of drug. Tracking the movement of endosomes to lysozomes or even the golgi body in the presence of MSD, for instance via the use of membrane dyes (Schroer et al., 1991), would provide a more in-depth understanding of the effects of microtubule stabilization on receptor mediated endocytosis.

Overall, we found that the microtubule stabilizer paclitaxel does not inhibit endocytosis of LDL or ac-LDL over 4 hours, even at higher doses of $10 \mu \mathrm{M}$. We also found that there was no significant difference in the amounts of LDL or ac-LDL taken up despite there being multiple receptors for the endocytosis of ac-LDL. Given that multiple ligands such as transferrin, protein hormones, lysosomal enzymes, certain viruses and even maternal immunoglobulins are endocytosed into the cell (Anderson et al., 1982), it would 
be beneficial to investigate the effects of microtubule stabilization on the later events of the endocytic process. These studies would be excellent in further developing our understanding of cellular transport, the role of microtubules and the effects of MSD treatment. 


\section{CHAPTER 5: BACTERIAL PHAGOCYTOSIS BY MSD TREATED RAW264.7 MACROPHAGES.}

\subsection{GFP-labelled E.coli and phagocytosis by RAW264.7 macrophages.}

\subsubsection{Introduction}

Apart from RME, another important endocytic pathway in macrophages is phagocytosis. Macrophages are specialized for phagocytosis and are central to two main immune functions. Firstly, they ingest pathogens and activate the microbial death pathway, which includes production of hydrolytic enzymes and inflammatory cytokines (Harrison et al., 2002; Underhill et al., 2002). Secondly, they are important in combination with dendritic cells (DC), to target antigens to MHC I and II complexes, thus bridging the innate and adaptive immune systems (Greenberg et al., 2002; Harrison et al., 2002). The phagocytic processes are initially actin-dependent, however upon formation of the phagosome, actin is shed and the phagosome matures along the microtubule network, resulting in fusion with the lysozome (Desjardins et al., 1994b; Aderem et al., 1999). Phagocytosis requires an interaction between functional actin filaments and microtubules, and functional dynamic microtubules are also required for antigen processing (Underhill et al., 2002; Peachman et al., 2004).

Phagocytosis is part of the process by which the immune system initiates defence against invading pathogens. Immune cell function can be diminished due to neutropenia or immunosuppression, for example during chemotherapy with paclitaxel or docetaxel (Rowinsky et al., 1995; Javeed et al., 2009). As mentioned previously, MSD treatment causes microtubule stabilization and it is possible that functions depending on microtubules would be impaired. For these reasons we were interested in studying the effects that MSD treatment had on the phagocytic capabilities of macrophages. Previous studies have looked 
at the MDD colchicine and its effect on phagocytosis, reporting that colchicine significantly depressed phagocytic capability in non-activated, non-elicited resident mouse macrophages (Khandani et al., 2007). Studies have also shown that treatment with paclitaxel led to decreased efficiency of complement-mediated phagocytosis (Lewkowicz et al., 2008). While MDD treatment causes microtubules to disperse and break down, MSD treatment causes microtubules to stabilize and we hypothesized that in the presence of stabilized microtubules, macrophages would display an impaired phagocytic ability. In addition, we wanted to investigate the killing ability of these MSD treated macrophages. We hypothesized that if phagocytosis itself was not impaired, it could be possible that MSD treated macrophages would be unable to kill the phagocytosed bacteria. Furthermore, it is known that paclitaxel has LPS mimicry, and is capable of increasing inflammatory cytokine production (Ding et al., 1990b), while peloruside A does not share this property (Crume et al., 2007). It is also known that paclitaxel and docetaxel have immunomodulatory effects and can regulate cytokine production (Chan et al., 2000). Based on the above information, we were also interested in the regulation and production of cytokines, especially TNF- $\alpha$, during phagocytosis in MSD treated macrophages.

There are various methods available for the study of phagocytosis. Originally methods included fluorescent labelling of particles, followed by quenching of extracellular fluorescence with trypan blue (Wan et al., 1993; Klippel et al., 2007). Other methods suggested repeated washing of cells after phagocytosis to detach extracellular particles, followed by lysis of the cell to release intracellular particles for quantification (Khandani et al., 2007). There are also a variety of methods that use live cell imaging, and flurogenic 
probes coupled with quantification via a spectrophotometer or flow cytometer (Russell et al., 2009). We wanted to study the uptake of live bacteria rather than uptake of particles such as IgG-opsonized sheep red blood cells (SRBC) or latex beads as different receptors are involved in recognition of differing particles (Takeda et al., 2003). In addition, cellular and immune responses can differ depending on the phagocytosed material. The activation state of macrophages and the amount of hydrolytic enzymes produced change depending on the presence of LPS and IFN- $\gamma$ stimuli (Yates et al., 2007). The use of live bacteria would stimulate macrophages similarly to an in vivo infection, leading to more efficient phagocytosis. It was also thought that to evaluate the true effects of MSD on phagocytosis, the assays should be as close as possible to real infections and this resulted in us choosing viable bacteria. Furthermore, we would be able to look into the immunomodulatory effects of MSD on cytokine production during bacterial killing. 


\subsubsection{Results}

Initially, GFP positive (GFP+ve) E.coli was used to infect RAW264.7 cells, following the methods described section 2. The GFP signal was not constitutive but was IPTG inducible. We also had access to GFP negative (GFP-ve) bacteria that allowed calibration of instruments, and confirmation that RAW264.7 cells phagocytosed both strains of bacteria similarly (Fig5.1.1). All cells were not stained for macrophage markers in the early assays as staining was carried out in FACS buffer containing sodium azide, which seemed to cause loss of GFP signal or inhibit survival of bacteria after two to four hours (Fig 5.1.2). However, macrophages alone stained for CD11b-PE were included as a control to set live cell and bacterial gates. We tested varying concentrations of bacteria to determine optimal multiplicity of infection (MOI). At low MOI, it was relatively easy to distinguish the two populations; however at higher MOI it was difficult to ascertain the macrophage population from the bacterial population (Fig 5.1.3).

We then stained macrophages using an Invitrogen cell tracker probe, cell tracker orange (5-(and-6)-(((4-chloromethly)benzoyl)amino)tetramethylrhodamine (CMTMR), as this stain did not require FACS buffer, ensuring maintenance of GFP+ve signal (Fig 5.1.4). However although we could distinguish bacterial and cell populations, it was not possible to determine internal phagocytosed bacteria from external bacteria adhered to the macrophages, especially with increasing MOI (Fig 5.1.4 and Fig 5.1.5).

The next method trialled to address the problem of differentiating intracellular vs extracellular bacteria was via the use of an antibiotic treatment that kills extracellular 
bacteria (Al-Mariri, 2008; Subramanian et al., 2008). After infection of macrophages, cells are treated with antibiotics such as gentamicin for 2 to 4 hours to kill or incapacitate extracellular bacteria. We used this antibiotic method to kill extracellular bacteria; however we found that gentamicin-killed bacteria were still fluorescent and expressed GFP similarly to live bacteria (Fig 5.1.6). We also trialled quenching of extracellular fluorescence via trypan blue, however this too was unsuccessful, as trypan blue is fluorescent itself causing a baseline fluorescence. In addition it seemed to mask the intracellular GFP signal. 


\section{Figure 5.1.1}

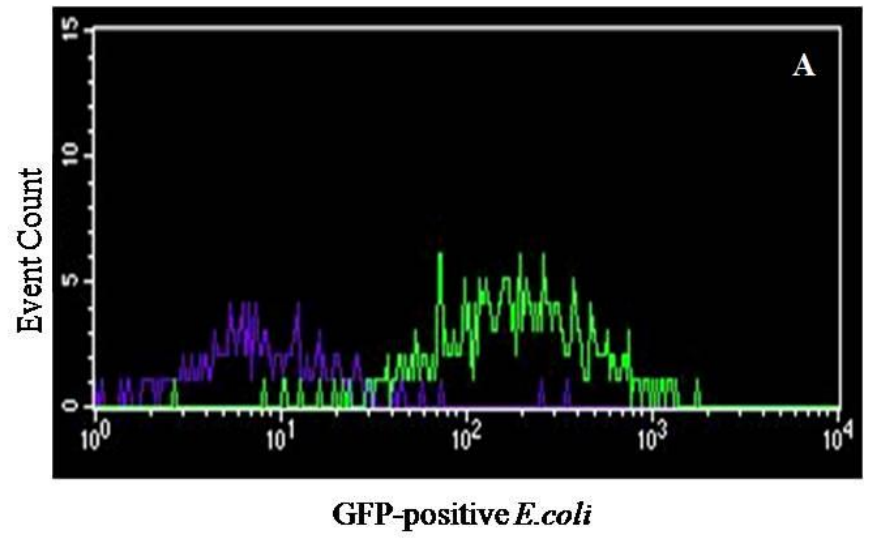

\subsubsection{Figure Legend}

- GFP negative bacteria

- GFP positive bacteria

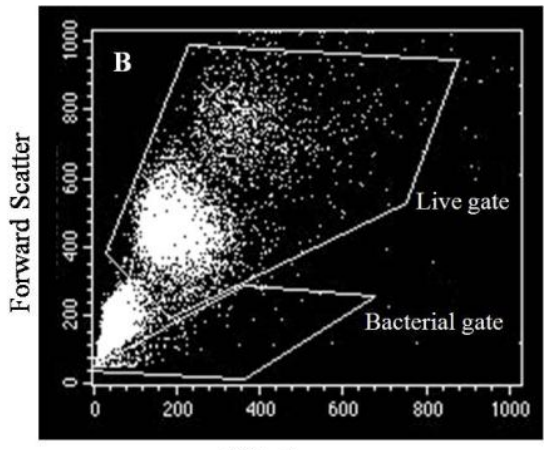

Side Scatter

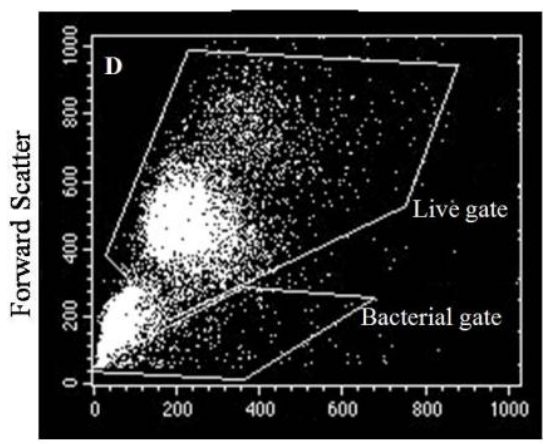

Side Scatter

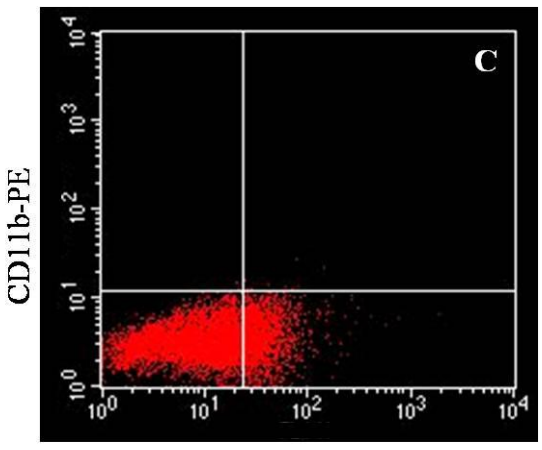

GFP-positive E.coli

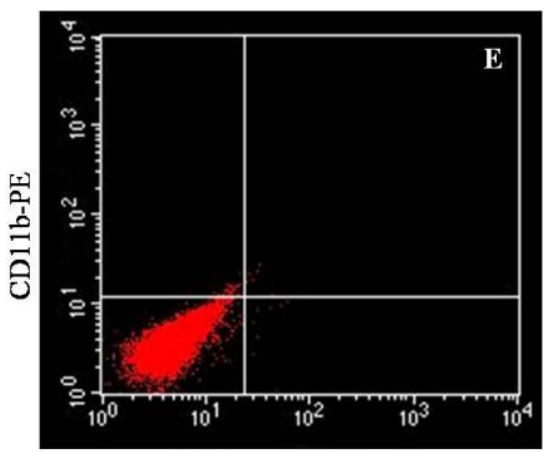

GFP-negative E.coli

Figure 5.1.1 Histogram and Flow cytometry plots showing GFP+ve and GFP-ve E.coli. (A) Positive and negative GFP E.coli are distinct populations (B, C) RAW264.7 macrophages phagocytose GFP+ve bacteria and show a shift in the lower right quadrant, compared to (D, E) RAW264.7 macrophages phagocytose GFP-ve bacteria but remain in the lower left quadrant, and are not GFP+ve. 


\section{Figure 5.1.2}

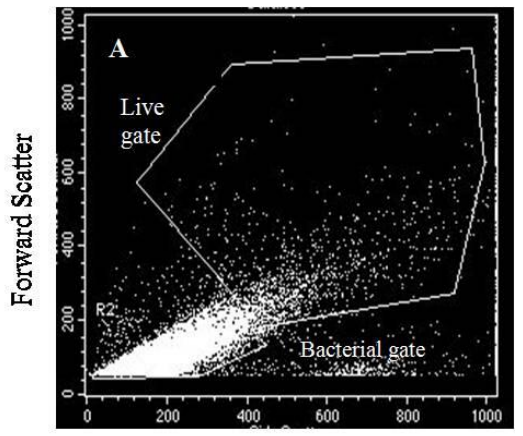

Side Scatter

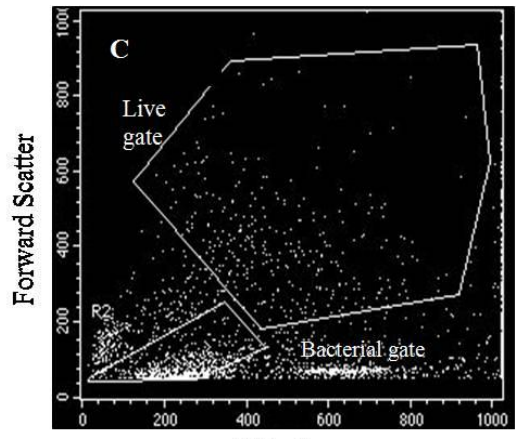

Side Scatter

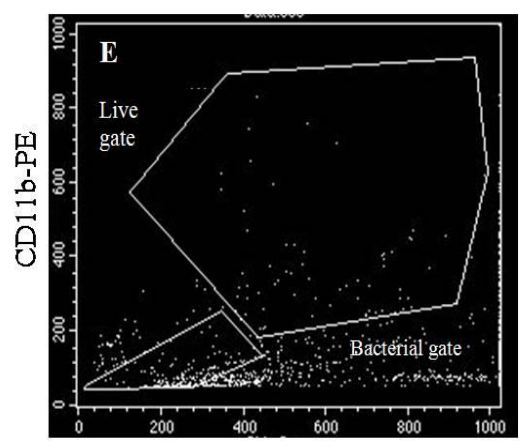

GFP-positive E.coli

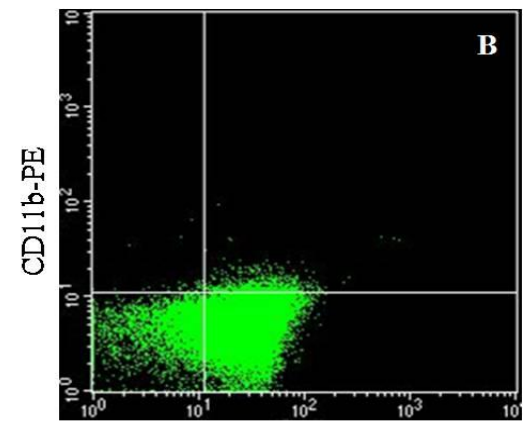

GFP-positive E.coli
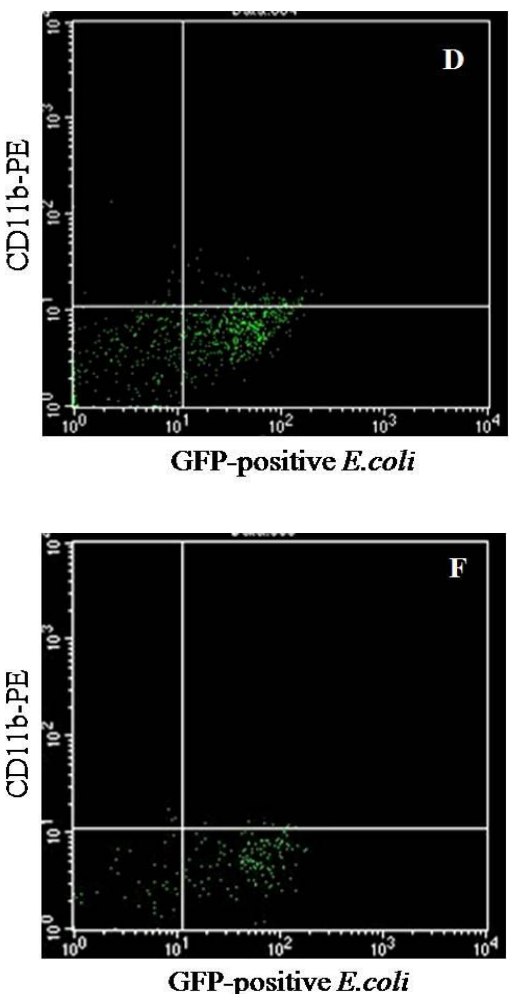

Figure 5.1.2 Flow cytometry plots showing effect of FACS buffer on GFP+ve E.coli.

(A) Bacterial population with a live cell and bacterial gate. (B) Bacteria survive in FACS buffer for 20 minutes and emit a strong GFP signal, compared to (C, D) bacteria in FACS buffer for 2 hours, with a decrease in bacterial count and fluorescence. (E, F) Bacteria are most affected after 4 hours in FACS buffer, GFP signal is low and the population has decreased. 
Figure 5.1.3

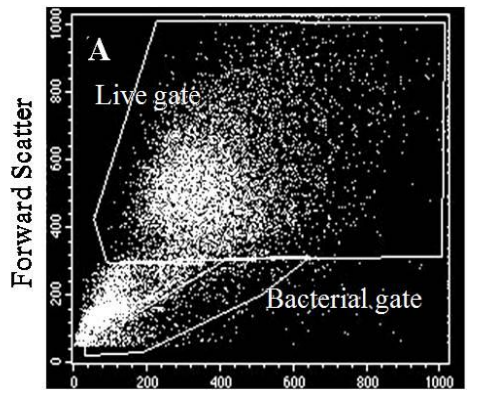

Side Scatter

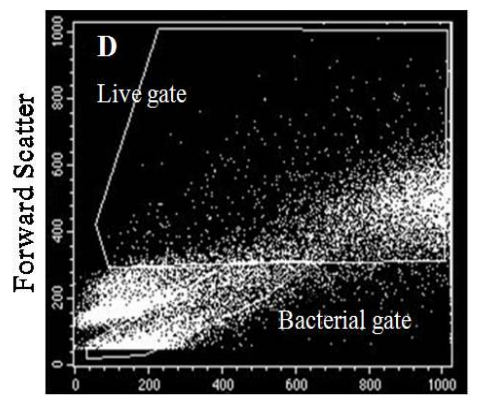

Side Scatter

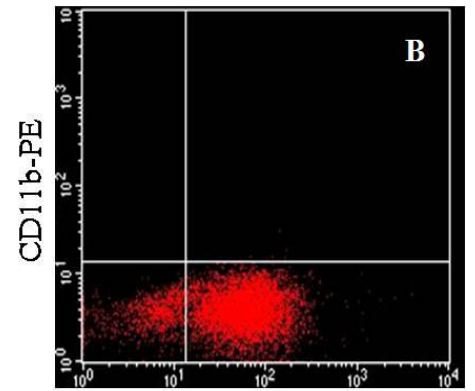

GFP-positive E.coli

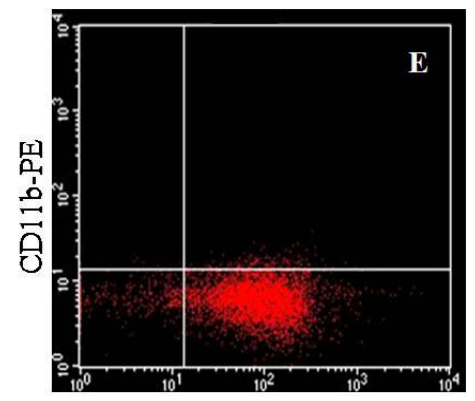

GFP-positive E.coli

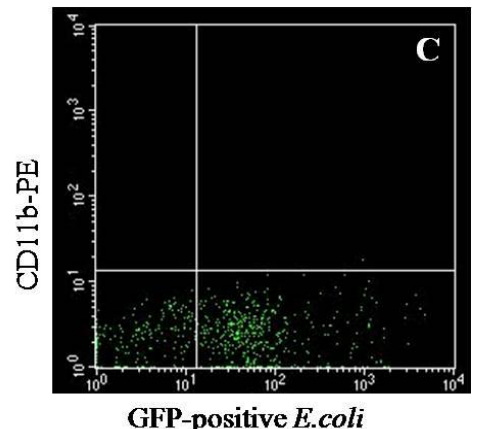

GFP-positive E.coli

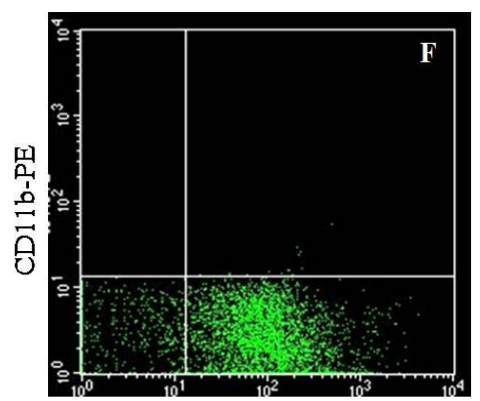

GFP-positive E.coli

Figure 5.1.3 Bacterial population is hard to distinguish from unstained macrophages, when bacteria are added at high MOI. (A, B, C) RAW264.7 macrophage population can be distinguished from GFP+ve bacteria at low MOI. FACS plot C shows low population of bacteria from bacterial gate, compared to $(\mathbf{D}, \mathbf{E}, \mathbf{F})$ where at high MOI bacteria and RAW264.7 macrophages are harder to distinguish. FACS plot F shows high population of bacteria from bacterial gate. 
Figure 5.1.4

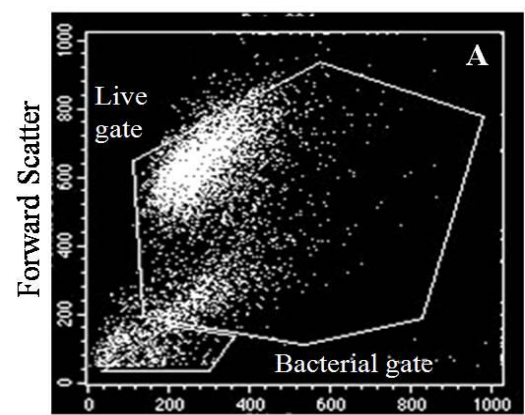

Side Scatter

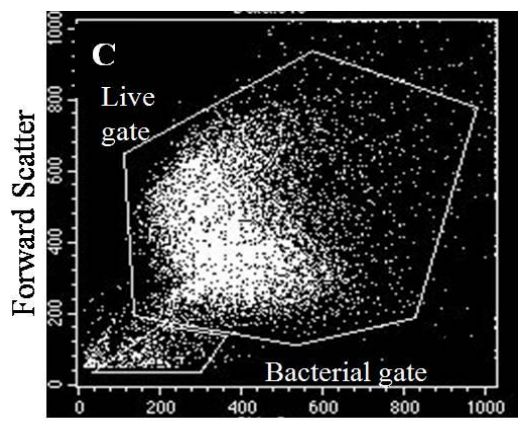

Side Scatter

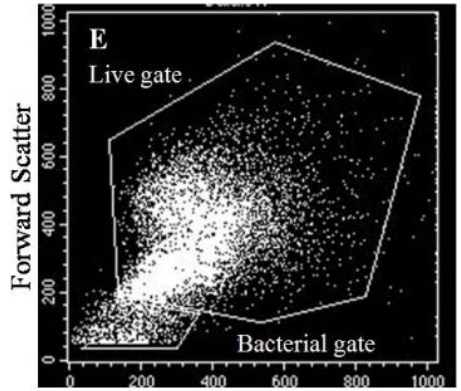

Side Scatter

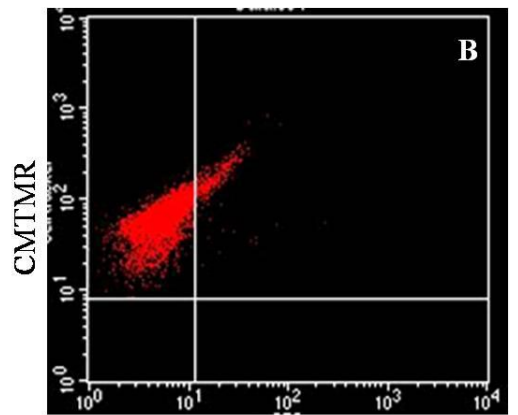

GFP-positive E.coli

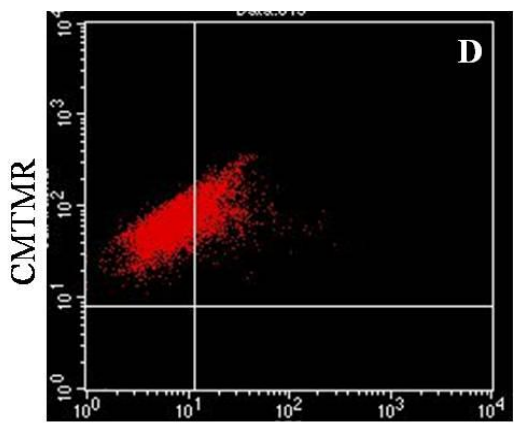

GFP-positive E.coli

Figure 5.1.4 CMTMR labelled macrophages and GFP+ve bacteria. (A, B) RAW264.7

macrophages stained with CMTMR. (C, D) Macrophages with low MOI bacteria cause a shift in the population making the cells double positive for CMTMR and GFP. (E, F, G) With higher MOI, it is harder to distinguish external bacteria from phagocytosed, internal bacteria. There is an increase in number of double positive live cells, however bacterial gate (G) also shows double positive cells, indicating the presence of mixed cells and bacteria. 


\section{Figure 5.1.5}

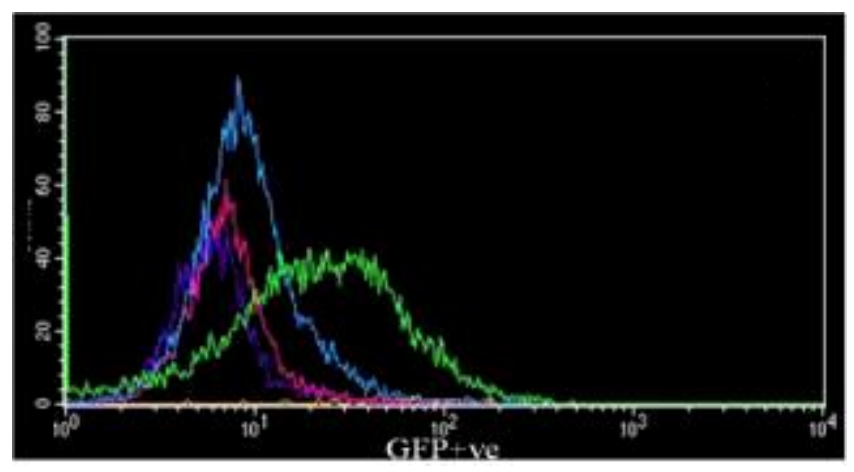

5.1.5 Figure Legend

- CMTMR stained cells

- CMTMR stained cells with

GFP+ve bacteria:

$\begin{array}{ll}\text { - } & \text { MOI 1:1 } \\ \text { - } & \text { MOI 10:1 } \\ \text { - } & \text { MOI 100:1 }\end{array}$

Figure 5.1.5 RAW264.7 cells incubated with increasing MOI of GFP+ve bacteria. Compared to MOI 1:1 and 10:1, at MOI of 100:1 there is an evident shift in the histogram. However it is not clear if bacteria are internalized or adherent and the increased fluorescence can be due to an increase in MOI. (MOI: multiplicity of infection of bacteria: macrophage)

Figure 5.1.6
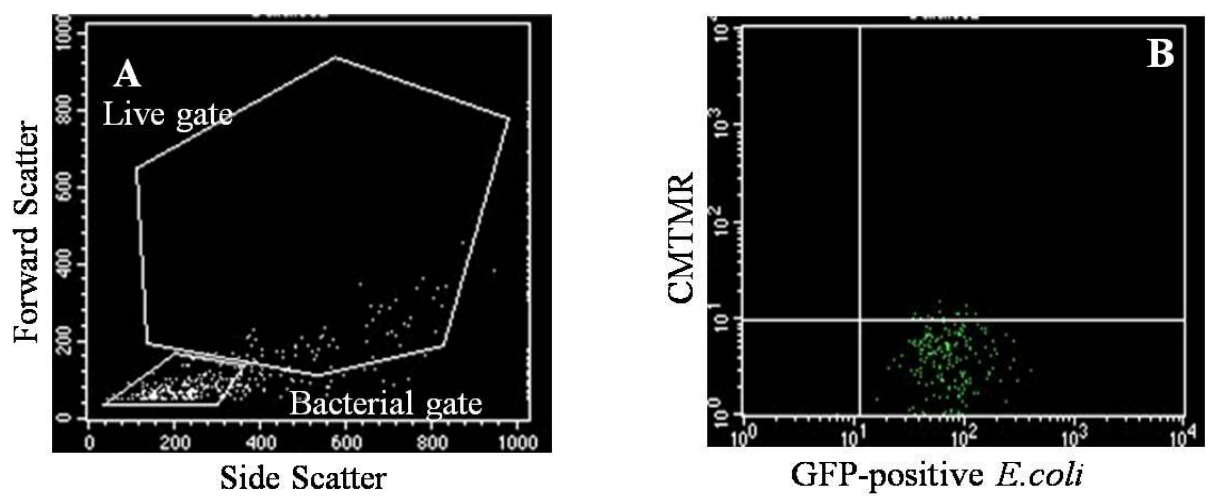

Figure 5.1.6 GFP+ve bacteria incubated with gentamicin are fluorescent and express a GFP+ve signal. Incubation of bacteria with $100 \mu \mathrm{g} / \mathrm{ml}$ of the antibiotic gentamicin, to facilitate bacterial killing, has no effect on the expression of the GFP signal. Bacteria are dead, however they still fluoresce, indicating that extracellular bacteria treated with gentamicin would still express GFP. 


\subsubsection{Discussion}

The use of GFP labelled bacteria to study phagocytosis was successful if the MOI was low. At low MOI, it can be assumed that all bacteria will be internalized and this can be confirmed via cytospins. However to study the effect of MSD treatment and microtubule stabilization on phagocytosis, it was essential to have the phagocytosis assays working with maximum bacteria. If MOI was low, then the effects seen with MSD treatment might be negligible as macrophages would still phagocytose bacteria. Also to mirror in vivo conditions, a higher MOI was preferred. We found that at higher MOI, it was increasingly difficult to distinguish between the macrophage and bacteria populations, especially since the macrophage populations were not stained. Staining of macrophages for markers such as CD11b and F4/80 was carried out in FACS buffer, and we found that this had a negative effect on survival of bacteria. A similar result was seen where sodium azide caused inhibition of E.coli phagocytosis in murine peritoneal macrophages (Miliukiene et al., 2007). Therefore using FACS buffer would skew the phagocytic results. Another problem with increasing MOI was the fact that high concentration bacterial cultures can have toxic effects on the macrophages (Ralph et al., 1977b).

The use of the cellular stain cell tracker orange (CMTMR) was helpful in differentiating between the two populations, however we once again had the problem of identifying intracellular and extracellular bacteria. Our initial aim was to identify inhibition or enhancement to phagocytic ability of MSD treated macrophages. To enable us to answer this aim, it was necessary to be able to differentiate internalized bacteria from adherent bacteria. The next method we trialled was using antibiotic treatments to kill extracellular bacteria. The idea behind this method was that once macrophages were infected and 
phagocytosis had occurred, use of an antibiotic to kill extracellular bacteria would aid in distinguishing intracellular and extracellular bacteria. However we found gentamicin-killed bacteria to be fluorescent, with the result being that we were still unable to identify phagocytosed bacterial populations successfully. It was also interesting to find that the trypan blue quenching protocol has reports of letdowns as well as success by different research groups (Peiser et al., 2000; Klippel et al., 2007).

Overall, use of GFP labelled bacteria and flow cytometry to study phagocytosis was unsuccessful for our experiments due to our requirement of needing to distinguish between internal bacteria and external bacteria. These methods are established methods and have been used successfully, especially in experiments where adherence and ingestion have together been regarded as uptake (Peiser et al., 2002; Areschoug et al., 2008).

Due to the limitations faced when using the above methods, we decided to explore other phagocytic assays that would allow differentiation between extracellular and intracellular bacterial populations. A recently developed dye from Invitrogen Molecular Probes $^{\circledR}$ proved to be an excellent option for the study of phagocytosis. The dye is a pHsensitive rhodamine-based dye, designed to allow specific determination of phagocytic events from non-specific binding. These assays are discussed in the following sections. 
Chapter 5: Bacterial phagocytosis by

MSD treated RAW264.7 macrophages.

\section{2 pHrodo E.coli bioparticles and phagocytosis by RAW264.7 macrophages.}

\subsubsection{Introduction}

As discussed in the previous section, one of the problems faced when studying phagocytosis is the difficulty in differentiating between internalized bacteria and adherent bacteria. In most cases, the previously discussed methods are sufficient; however, they may overestimate the actual engulfment of bacteria due to the detection of surface-bound bacteria. To determine the true engulfment of bacteria, we employed a novel flurogenic approach to distinguish phagocytic events from non-specific bound bacteria. The flurogenic pHrodo reagent is a rhodamine based dye that has low or no fluorescence at neutral $\mathrm{pH}$ and dramatically increases its fluorescence with a drop in $\mathrm{pH}$ (Fig 5.2.1) (Invitrogen, Molecular Probes). This system measures endocytosis or phagocytosis based upon acidification of ingested particles. Extracellular or bound particles do not fluoresce, thereby giving a direct measure of phagocytic events. pHrodo E.coli bioparticles have been used to study phagocytosis successfully (Lambert et al., 2008), while pHrodo-SE dye has been used to label apoptotic thymocytes, which are then used as phagocytic targets (Miksa et al., 2009). Thus this novel reagent can be used in various applications to study RME or phagocytosis.

We used pHrodo-labelled E.coli bioparticles ${ }^{\circledR}$ (hereafter referred to as pHrodo or pHrodo particles) to investigate the phagocytic ability of MSD treated macrophages. Once particles are phagocytosed, they start to fluorescence, however upon fusion of the phagosome with the lysozome, the $\mathrm{pH}$ drops further due to hydrolytic enzymes in the lysozome (Desjardins et al., 1994a; Luzio et al., 2007). The drop in $\mathrm{pH}$ leads to increasing intensity of fluorescence, so over time as more bacteria are phagocytosed and phagosomes fuse with lysozomes, there is increasing fluorescence seen within macrophages. 


\section{Figure 5.2.1}

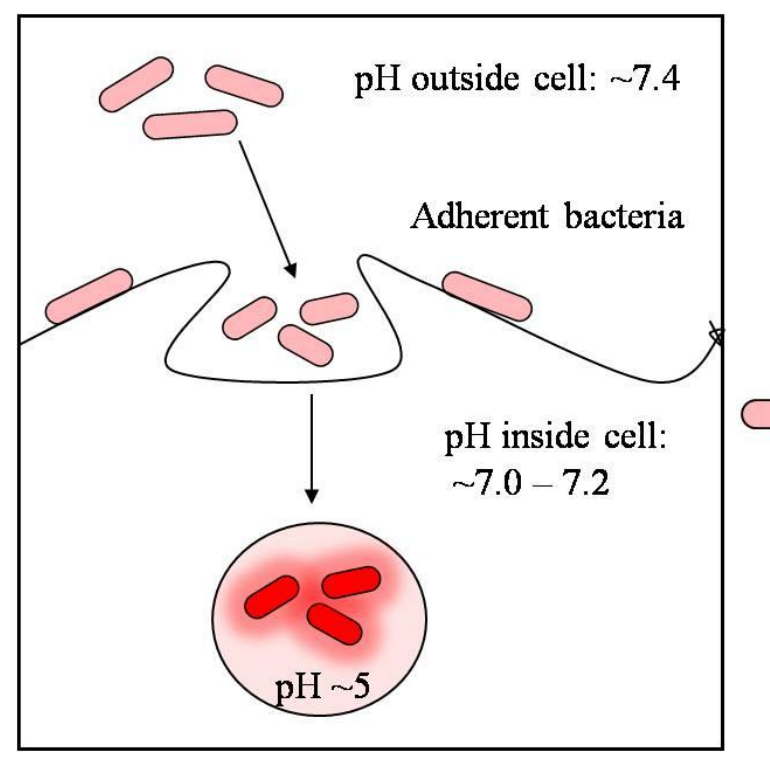

pHrodo labelled bacteria

Bacteria fluoresce brightly in low $\mathrm{pH}$ of phagocytic vesicles

Figure 5.2.1 Increase in fluorescence of phagocytosed pHrodo E.coli bioparticles. E.coli labelled with pHrodo $^{\mathrm{TM}}$ dye are added to macrophages. Non-fluorescent bacteria display an increase in fluorescence intensity once phagocytosed. Extracellular and adherent bacteria are non-fluorescent.

Adapted from Invitrogen "pHrodo ${ }^{\mathrm{TM}}$ indicators - enlightening phagocytosis" distribution poster. 
Chapter 5: Bacterial phagocytosis by

MSD treated RAW264.7 macrophages.

\subsubsection{Effects of MSD treatment on pHrodo phagocytosis in RAW264.7} macrophages.

We initially tested pHrodo with un-treated RAW264.7 macrophages to optimize culture conditions and concentrations. RAW264.7 macrophages were stained with carboxyfluorescein diacetate succinimidyl ester (CFDA-SE or CFSE) and phagocytic assays were carried out as described in the methods. Figure 5.2.2 shows live gated plots of CFSE stained RAW264.7 macrophages and baseline fluorescence of the pHrodo particles. The increase in mean fluorescence intensity (MFI) of pHrodo from baseline fluorescence is seen with the macrophages and pHrodo co-incubation plots. Due to the pHrodo being nonfluorescent when extracellular, distinguishing between the two populations was possible and actual phagocytic events were quantified.

Optimization of pHrodo uptake enabled us to use this system to investigate effects of MSD treatment on the phagocytic ability of macrophages. As mentioned earlier, there are few studies that investigate the effects of MSD on macrophage functions that also depend on microtubules. Our aim was to determine if treatment with MSD caused any inhibition or impairment to the macrophage ability to phagocytose bacteria. We hypothesised that MSD treatment would cause a reduction in the ability of macrophages to phagocytose bacteria. However, it was possible that due to the immunostimulatory properties of some of these MSD, macrophage phagocytic ability would be enhanced. We were also interested in the cytokine profile expressed by MSD treated macrophages during phagocytosis. LPS and paclitaxel cause an increase in TNF- $\alpha$, while this inflammatory profile is absent with peloruside A treatment (Ding et al., 1990b; Crume et al., 2007). With these aims in mind, we tested the effects of $3 \mathrm{MSD}$, namely paclitaxel, docetaxel and peloruside $\mathrm{A}$, on 
macrophages phagocytosis. Latrunculin A disrupts the actin cytoskeleton and inhibits phagocytosis (Coue et al., 1987; Spector et al., 1989; Oliveira et al., 1996). We used this drug in our studies as a positive control to ensure inhibition of phagocytosis. While paclitaxel, docetaxel and peloruside A are the 3 main MSD studied in this thesis, we also had limited access to other novel compounds. Zampanolide, mycothiazole and ixabepilone are also studied during various sections of this thesis depending on their availability.

\subsubsection{Effects of the novel compounds; zampanolide, ixabepilone (Ixempra ${ }^{\circledR}$ )} and mycothiazole on RAW264.7 macrophages during pHrodo phagocytosis.

Zampanolide is a potent microtubule stabilizer that blocks the cell cycle in the $G_{2} / M$ phase and shows cytotoxic activity against several tumour cell lines (Tanaka et al., 1996; Field et al., 2009). Ixabepilone is a semi synthetic derivative of epothilone B and it is the only epothilone with FDA approval for clinical use (Conlin et al., 2007; Goodin, 2008). Epothilones stabilize microtubules similarly to paclitaxel (Altmann, 2003). However, they are also competitive inhibitors of paclitaxel binding to microtubules, suggesting that paclitaxel and epothilone could share a binding site and have similar affinities for microtubules (Bollag et al., 1995; Goodin et al., 2004). Ixabepilone is used clinically in combination or mono-therapies for the treatment of metastatic or locally advanced breast cancers that are resistant to taxanes (Donovan et al., 2008; Goodin, 2008; Lechleider et al., 2008). Mycothiazole is sourced from a Fijian marine sponge, Cacospongia mycofijiensis (Sonnenschein et al., 2006). It shows considerable bioactivity and is selective against several tumour cell lines, particularly lung cancer. Recent studies have indicated that mycothiazole could inhibit activation of hypoxia inducible factor 1 (HIF-1) in tumor cells 
and at low nM concentrations, it could inhibit hypoxia induced vascular endothelial growth factor (VEGF) (Sashidhara et al., 2009). While the main MSD studied in this thesis are paclitaxel, peloruside A and docetaxel, we also tested the effects of these novel compounds on phagocytosis. 


\subsubsection{Results}

\section{Figure 5.2.2}

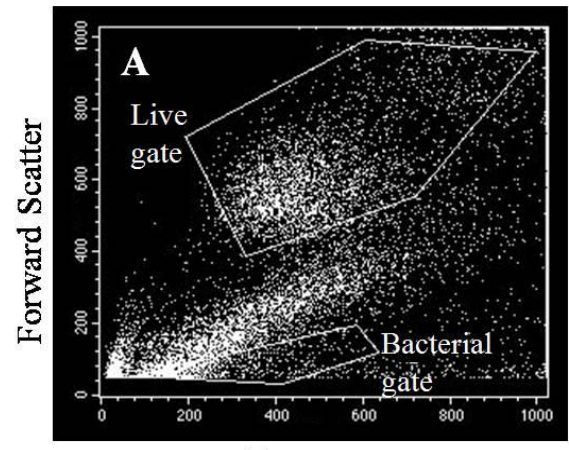

Side Scatter

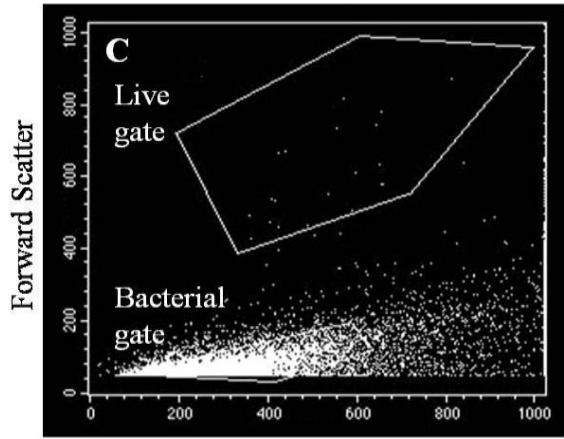

Side Scatter

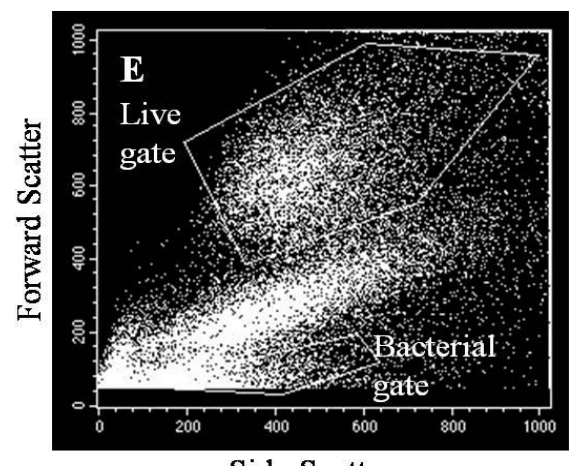

Side Scatter

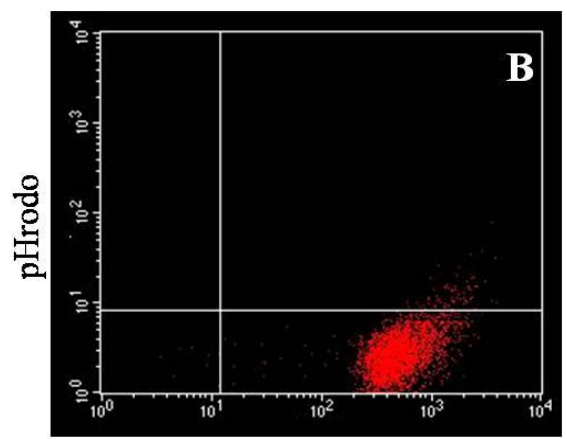

RAW264.7 CFSE

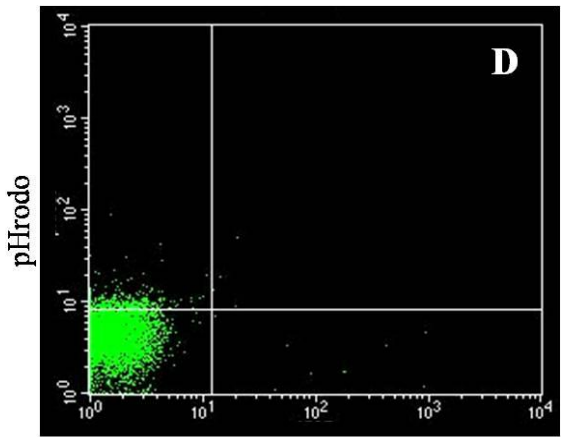

RAW264.7 CFSE

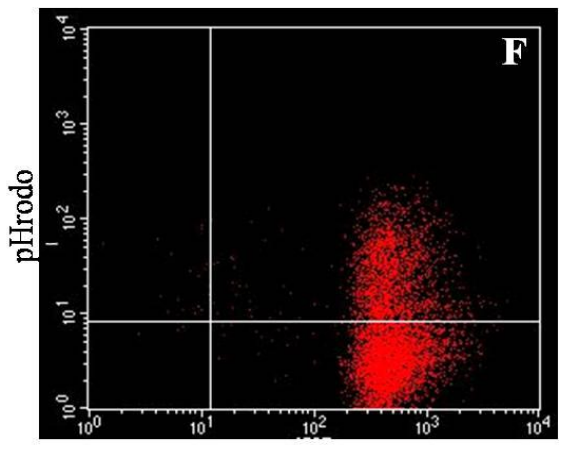

RAW264.7 CFSE

Figure 5.2.2 pHrodo particles show an increase in fluorescence when phagocytosed by macrophages. (A, B) Live gated macrophages are CFSE positive. (C, D) pHrodo particles alone have low baseline fluorescence. (E, F) Increase in fluorescence seen with addition of pHrodo to macrophages. All samples were incubated for 2 hours at $37^{\circ} \mathrm{C}$ before being collected on the flow cytometer. 


\section{Effects of MSD treatment on pHrodo phagocytosis in RAW264.7 macrophages.}

Macrophages were stained with CFSE and treated with low $(0.1 \mu \mathrm{M})$ and high $(1 \mu \mathrm{M})$ doses of MSD as described in methods. Pretreatment with IFN- $\gamma$ was also carried out as noted in figures. Following a 45 minute drug treatment, pHrodo was added to cells. Drug concentration was maintained at all times following addition of particles. Ethanol vehicle controls and un-treated macrophages infected with pHrodo particles were included as controls. A no-cell, pHrodo particle sample was also run to measure base-line fluorescence (Fig 5.2.3). Data is expressed as percentage of cells positive for pHrodo phagocytosis (Fig 5.2.4), or the MFI of the positive cells (Fig 5.2.5). Compared to vehicle controls, treatment of macrophages with paclitaxel, peloruside A or docetaxel did not significantly affect the phagocytic ability. However as compared to vehicle treated cells, treatment with high dose zampanolide and Latrunculin A significantly inhibited phagocytosis (Fig 5.2.4). The MFI values from low and high dose zampanolide treatment were significantly lowered compared to controls (Fig 5.2.5). 
Figure 5.2.3
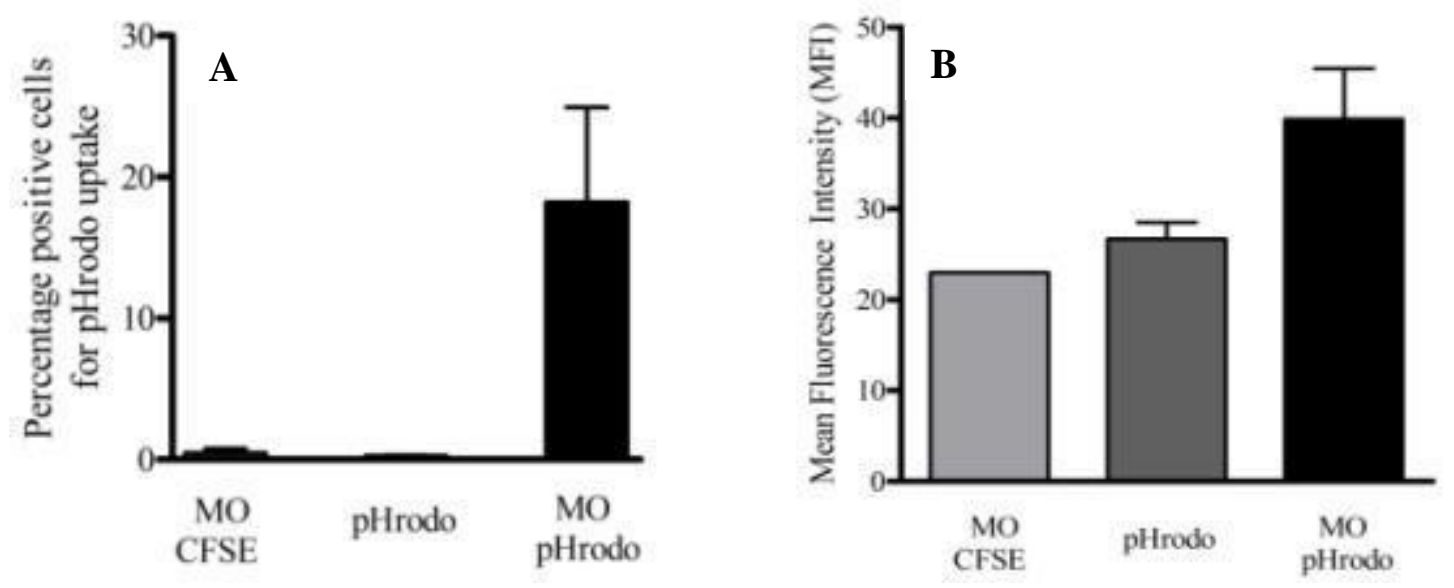

Figure 5.2.3 Macrophages (MO) and pHrodo alone show low percent positive cells; however intensity of fluorescence is high. (A) Macrophages (MO) and pHrodo together have increased fluorescence in comparison to either macrophages or pHrodo alone. Data is presented as mean percent positive cells for pHrodo uptake \pm SEM. There is no significant difference between the levels of fluorescence seen with the three samples. $p>0.05$, one-way ANOVA with Bonferroni post test. (B) MFI of positive cells indicates the fluorescence intensity of the positive cells. Macrophages alone and pHrodo alone have high MFI, indicating the positive cells are brightly stained, and the baseline fluorescence is high. Therefore while macrophages and pHrodo together have higher fluorescence levels, there is no significant difference between the levels of fluorescence seen with the three samples. $\mathrm{p}>0.05$, one-way ANOVA with Bonferroni post test. 
Figure 5.2.4

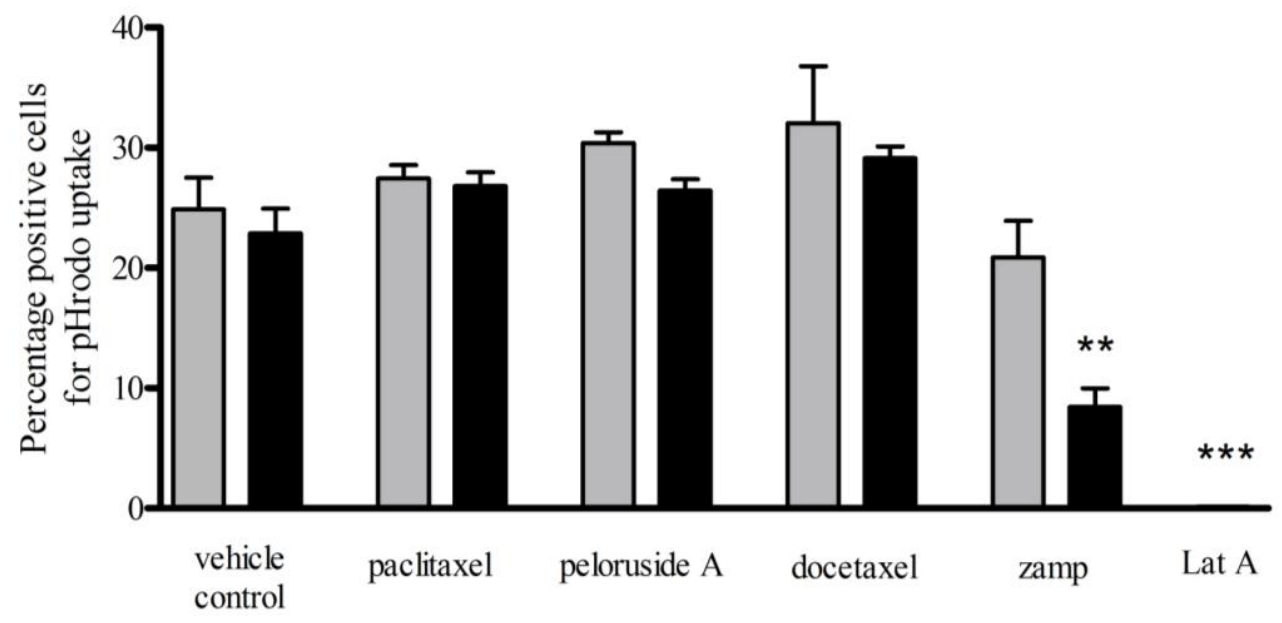

Figure 5.2.4 Phagocytosis of pHrodo by MSD treated macrophages. Cells were pretreated with $100 \mathrm{U} / \mathrm{ml}$ IFN- $\gamma$ overnight, followed by an MSD or equal concentration of ethanol (vehicle) pre-treatment for 45 minutes and incubation with pHrodo as described in methods. pHrodo uptake is expressed as the percent of cells positive for pHrodo uptake. Compared to vehicle controls, paclitaxel, docetaxel and peloruside A do not significantly inhibit phagocytosis. There was a non-significant decrease in the level of phagocytosis of low dose zampanolide treated cells and a significant decrease in the level of phagocytosis of high dose zampanolide treated cells. Bars represent mean \pm SEM from two independent experiments, except zampanolide which is mean \pm SEM from one experiment. Gray bars represent $0.1 \mu \mathrm{M}$ (low dose) MSD treatment/vehicle control; black bars $\square$ represent $1 \mu \mathrm{M}$ (high dose) MSD treatment/vehicle control. Lat A: latrunculin A. $* * \mathrm{p}<0.01$ zampanolide vs vehicle control, *** $\mathrm{p}<0.0001$ Latrunculin A vs vehicle control, one-way ANOVA with Bonferroni correction. 
Figure 5.2.5

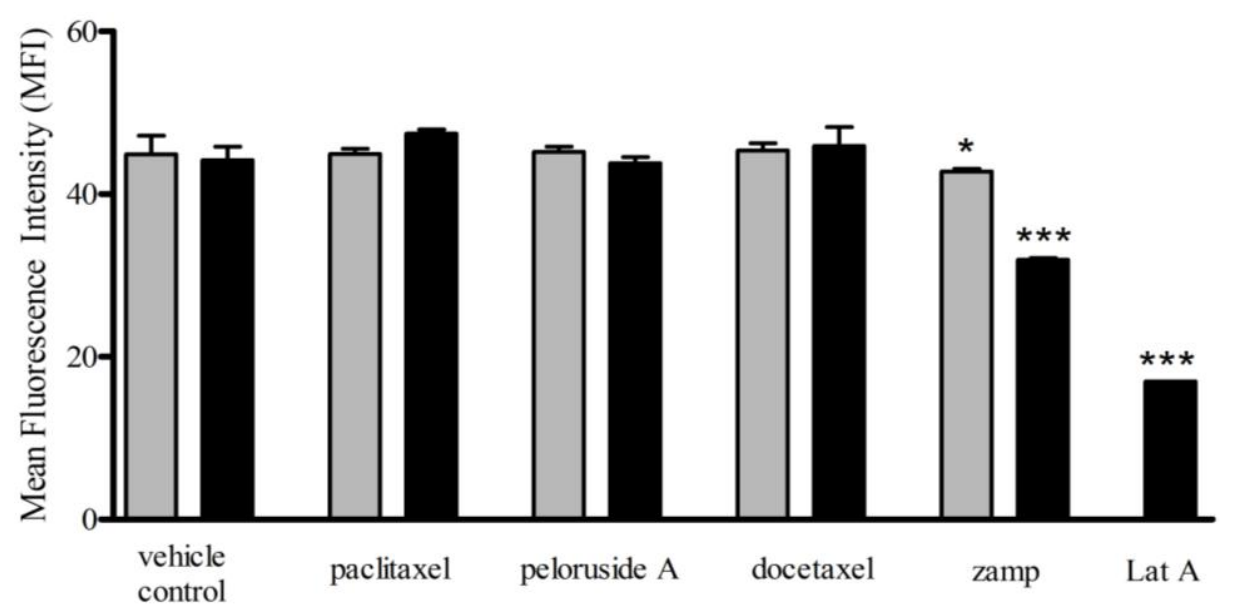

Figure 5.2.5 Changes in fluorescence intensity of cells positive for pHrodo uptake in the presence of MSD treatment. There was no change in the fluorescence intensity of paclitaxel, docetaxel or peloruside A treated macrophages. However compared to vehicle controls, zampanolide and Latrunculin A treated cells exhibited a significant decrease in fluorescence intensity. pHrodo uptake is expressed as mean fluorescence intensity \pm SEM of the cells positive for pHrodo uptake. Gray bars $\square$ represent $0.1 \mu \mathrm{M}$ (low dose) MSD treatment/vehicle control; black bars $\square$ represent $1 \mu \mathrm{M}$ (high dose) MSD treatment/vehicle control. Data is representative of 2 experiments, except for zampanolide which is representative of 1 experiment. Zamp: zampanolide, Lat A: latrunculin A. *p<0.05 low dose zampanolide vs low dose vehicle control, ***p<0.001 high dose zampanolide vs high dose vehicle control, ***p<0.001 Latrunculin A vs high dose vehicle control, one-way ANOVA with Bonferroni correction. 
Effects of ixabepilone and mycothiazole treatment on pHrodo phagocytosis in RAW264.7 macrophages.

Macrophages were stained with CFSE and treated with low $(0.1 \mu \mathrm{M})$ or high $(1 \mu \mathrm{M})$ doses of either ixabepilone or mycothiazole as described in methods. Pretreatment with IFN- $\gamma$ was also carried out as noted in figures. Following a 45 minute drug treatment, pHrodo was added to cells. Drug concentration was maintained at all times following addition of particles. Vehicle (ethanol and DMSO) treated samples were included. The experiments with ixabepilone, mycothiazole and latrunculin were carried out together; however data is presented in 2 figures as the vehicle control for mycothiazole is DMSO, while the vehicle control for ixabepilone is ethanol. Data is expressed as percentage of cells positive for pHrodo phagocytosis (Fig 5.2.6), or the MFI of the positive cells (Fig 5.2.7). Compared to vehicle controls, treatment of macrophages with ixabepilone or mycothiazole at either dose, did not significantly affect the phagocytic ability. While with mycothiazole treatment a low dose DMSO control was not included, the high dose DMSO treatment had no effect on phagocytic ability and so we assume that the low dose vehicle would not affect phagocytosis and the results would be similar to phagocytic levels seen in high dose DMSO treated cells (Fig 5.2.6 and 5.2.7). Treatment with the positive control Latrunculin A significantly inhibited phagocytosis. 
Figure 5.2.6
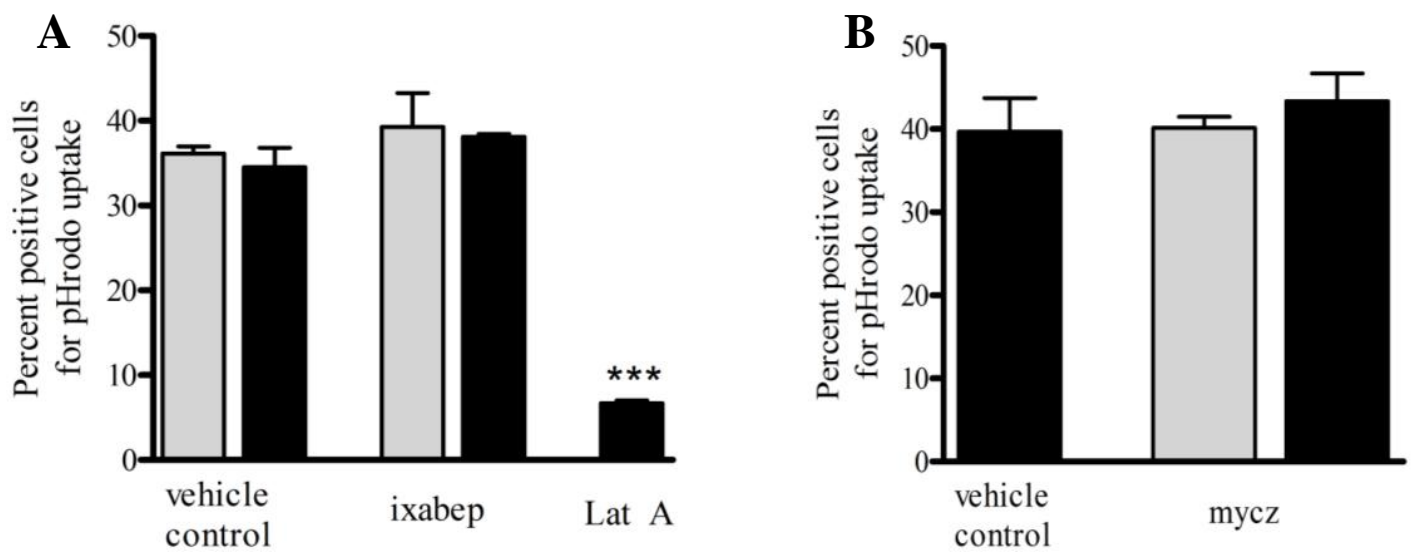

Figure 5.2.6 Phagocytosis of pHrodo in the presence of (A) ixabepilone and (B)

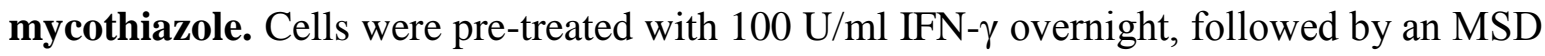
or equal concentration of ethanol or DMSO (vehicle) pre-treatment for 45 minutes and incubation with pHrodo as described in methods. pHrodo uptake is expressed as the percent of cells positive for pHrodo uptake. Compared to vehicle controls, except for Latrunculin A, there is no significant inhibition of phagocytosis in (A) ixabepilone or (B) mycothiazole treated macrophages. Bars represent mean \pm SEM from one experiment. (A) Gray bars represent $0.1 \mu \mathrm{M}$ (low dose) ixabepilone/ethanol control; black bars $\quad$ represent $1 \mu \mathrm{M}$ (high dose) ixabepilone/ethanol control. (B) Bars represent $0.1 \mu \mathrm{M}$ (low dose) $\square$ and 1 $\mu \mathrm{M}$ (high dose) $\square$ mycothiazole treatment and its DMSO vehicle control $\square$. Lat A: latrunculin A. ***p<0.001 Latrunculin A vs vehicle control, one-way ANOVA with Bonferroni correction. 
Figure 5.2.7
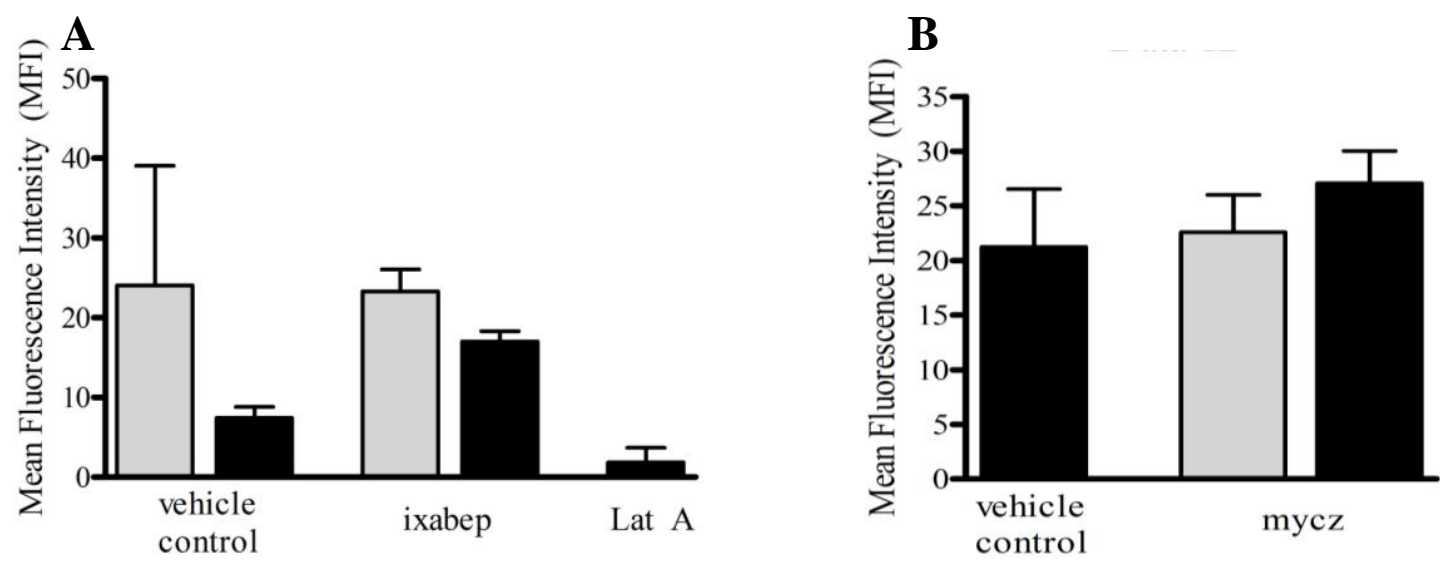

Figure 5.2.7 Changes in fluorescence intensity of cells positive for pHrodo uptake in the presence of (A) ixabepilone and (B) mycothiazole. Compared to vehicle controls, there is no significant change in the fluorescence intensity of any of the treated macrophages. pHrodo uptake is expressed as mean fluorescence intensity \pm SEM of the cells positive for pHrodo uptake. (A) Gray bars $\square$ represent $0.1 \mu \mathrm{M}$ (low dose) ixabepilone/ethanol control; black bars represent $1 \mu \mathrm{M}$ (high dose) ixabepilone/ethanol control. (B) Bars represent $0.1 \mu \mathrm{M}$ (low dose) $\square$ and $1 \mu \mathrm{M}$ (high dose) $\square$ mycothiazole treatment and its DMSO vehicle control . Lat A: latrunculin A. Data is representative of 1 experiment. $\mathrm{p}>0.05$, one-way ANOVA with Bonferroni correction. 


\subsubsection{Discussion}

This set of experiments was aimed at investigating the effects of MSD treatment on macrophage phagocytic ability. We hypothesized that treatment with MSD would lead to an inhibition of macrophage phagocytic ability. In comparison to vehicle treated cells, we found that treating macrophages with paclitaxel, docetaxel or peloruside A did not significantly affect the phagocytic ability. There was no enhancement or impairment to phagocytosis of pHrodo. With low dose zampanolide treated cells however, there was a non-significant decrease in phagocytosis of pHrodo. High dose zampanolide treated cells showed a significant impairment to phagocytosis via a decrease in pHrodo uptake. Latrunculin A is an actin destabilizer and a known phagocytic inhibitor (Oliveira et al., 1996) and as expected, we saw significant impairment to phagocytic processes in Latrunculin A treated cells.

It was interesting to find that zampanolide treated cells had impaired phagocytosis. Zampanolide is a potent MSD (Field et al., 2009; Miller et al., 2010), however the other MSDs used in this study did not show any decrease in phagocytic ability. A possible explanation for this could be related to the fact that zampanolide is active in cells resistant to paclitaxel (Field et al., 2009). The $\mathrm{IC}_{50}$ data presented in chapter 3, illustrates that RAW264.7 cells are relatively resistant to paclitaxel. It is possible that RAW264.7 cells are extremely susceptible to zampanolide. Additionally, in context of MSD dosage, doses of $0.1 \mu \mathrm{M}$ and $1 \mu \mathrm{M}$ are relatively high. The combination of drug susceptibility and high doses could have potentially caused the significant decrease in phagocytosis. It would be interesting to look at the potency of zampanolide in RAW264.7 cells and understand its cytotoxic effects in this cell line. 
While it is known that zampanolide binds to tubulin, the binding site is unknown (Miller et al., 2010) and zampanolide could potentially also have additional activity on actin. Zampanolide has been isolated by two separate groups, from a collection of sponges that had previously generated a number of bioactive compounds, including laulimalide and Latrunculin A (Tanaka et al., 1996; Miller et al., 2010). Zampanolide treated macrophages showed a decrease in phagocytic ability similar to that seen with Latrunculin A treated macrophages, suggesting that there may be effects on microtubules as well as on the actin cytoskeleton. The fact that phagocytosis is initially dependent on actin (Aderem et al., 1999; Underhill et al., 2002) also adds to the possibility that zampanolide has some actin related effects.

We found no impairment to phagocytic activity in macrophages treated with ixabepilone or mycothiazole as compared to the vehicle controls. Ixabepilone is a MSD with activity similar to that of paclitaxel (Bollag et al., 1995) and so it is not surprising that treatment with ixabepilone did not inhibit macrophage phagocytosis. Mycothiazole's activity is still unknown and this compound has not been classified as yet (Miller et al., 2010). However, the pattern of un-altered phagocytosis in mycothiazole treated macrophages suggests that mycothiazole may have a microtubule stabilizing activity similar to that of the other MSDs tested.

When looking at pHrodo phagocytosis, we measured the percent of cells positive for pHrodo uptake, but we also measured the mean fluorescence intensity (MFI) for these percent positive cells. At this point, if the CFSE cells alone were autofluorescent or had high levels of fluorescence, this was subtracted from MFI of pHrodo and macrophages together to remove the effect of baseline fluorescence. The MFI value usually relates 
intensity of fluorescence to the amount of ligand or fluorescent particles taken up. Therefore a high MFI indicates high amount phagocytosis for those cells. However with the use of pHrodo care needs to be taken when interpreting the MFI data. Since pHrodo is $\mathrm{pH}$ sensitive, as $\mathrm{pH}$ drops the intensity of fluorescence increases dramatically. Phagosomes have a lower $\mathrm{pH}$ compared to the cell cytoplasm resulting in fluorescence of pHrodo. However, when phagosomes fuse with the lysozomes, there is a further drop in $\mathrm{pH}$ due to presence of hydrolytic enzymes (Luzio et al., 2007) and this can cause an increase in fluorescence intensity. Thus with pHrodo phagocytosis an increase in fluorescence intensity does not necessarily relate to an increase in uptake, but could indicate a greater number of phagosomes fusing with lysozomes.

The MFI values of macrophages treated with paclitaxel, docetaxel, peloruside A and vehicle control were all similar, indicating that pHrodo uptake and fluorescence was similar. In contrast, the MFI of zampanolide treated macrophages was significantly lower, which supports the result of less pHrodo uptake due to impaired phagocytosis. The MFI results of Latrunculin A treated cells showed that even though percent of cells positive for pHrodo uptake was extremely low, the MFI was still high, in relation the the amount of pHrodo phagocytosed. This indicates that there was probably a degree of pHrodo uptake and those particles fluoresced especially brightly. The MFI results of mycothiazole treated cells are similar to the vehicle control and along with the percent positive cells indicate that uptake was not impaired. The high dose ixabepilone treated macrophages had high percent positive cells, but low MFI indicating that per each cell, fluorescence of pHrodo was lower. This could possibly indicate an inability to fuse with lysozomes or inhibition in lysosomal trafficking, however a similar result was seen with the high dose ethanol vehicle control, 
where positive cells were high, but MFI level was low. Taken together, it is unlikely that ixabepilone treated macrophages have impaired lysozomal fusion or trafficking. Tracking of the pHrodo particles once phagocytosed would be worth studying to understand if trafficking to the lysozome is affected. Studies found that phagolysosome formation in colchicine treated macrophages was not impaired (Pesanti et al., 1975), which is interesting since colchicine is an MDD that disrupts microtubule stability. This suggests that microtubules are not required for trafficking of lysozomes during phagolysosomal fusion and that this process occurs in a microtubule independent fashion. It would be interesting to look at phagolysosome formation in presence of MSD treatment, which would help to understand if later events such as stimulation of the adaptive immune response, are affected by MSD treatment.

Overall we found that treating macrophages with paclitaxel, docetaxel and peloruside A did not impair phagocytosis. We did not find any enhancement to phagocytic ability in these MSD treated macrophages either, indicating that the LPS mimicry and immunostimulatory effects of paclitaxel does not lead to an increase in phagocytic efficiency. With the novel MSD zampanolide, we saw a significant decrease in phagocytic ability at high doses, while there was a trend of impaired phagocytosis at the lower dose of zampanolide. Mycothiazole did not impair phagocytosis, suggesting that mycothiazole could possibly bind and stabilize microtubules similar to paclitaxel and the other MSD. Ixabepilone results were similar to results from the other MSDs and indicated that MSD treatment does not affect phagocytosis of bacteria. While this indicates that the uptake of bacteria is not impaired in the presence of MSD therapy, the processing of bacterial 
components and antigen presentation to activate the adaptive immune system, both of which require functional microtubules (Peachman et al., 2004), could potentially be impaired resulting in an inability to respond to infections. 
Chapter 5: Bacterial phagocytosis by

MSD treated RAW264.7 macrophages.

\subsection{Interactions between MSD and INF- $\gamma$ during pHrodo phagocytosis.}

\subsubsection{Introduction}

IFN- $\gamma$ is an important cytokine that stimulates macrophages to produce antimicrobial and antitumor mechanisms as well as causing an up-regulation of antigen presentation and processing pathways via induction of MHC pathways (Young et al., 1995; Schroder et al., 2006). IFN- $\gamma$ stimulus also results in the up-regulation of $\mathrm{FcR} \gamma$, complement proteins that aid in opsonization of pathogens, and complement receptors such as Mac-1 that enable responses to microbial and intracellular pathogens (Schroder et al., 2004). IFN- $\gamma$ is also known to synergize with LPS and promote production of nitric oxide (NO) (Young et al., 1995). LPS stimulation in macrophages induces secretion of IL-12 and chemokines which attract natural killer cells (NKC) to the site of inflammation, where IL-12 induces production of IFN- $\gamma$ in the NKCs. Further to this, IFN- $\gamma$ and IL-12 coordinate the link between pathogen recognition by the innate immune system, and induction of specific adaptive immune responses by promoting Th1 responses (Schroder et al., 2004). Based on the above details, when setting up phagocytosis assays, we primed macrophages overnight with $100 \mathrm{U} / \mathrm{ml} \mathrm{IFN}-\gamma$ to enhance phagocytic responses.

However, a recent study has found that priming macrophages with IFN- $\gamma$ led to protection from colchicine inhibition of phagocytosis (Khandani et al., 2007). The researchers found that resistance to microtubule de-stabilizers was related to IFN- $\gamma$ activation of macrophages, which increased microtubule stability and caused activated macrophages to exhibit a high proportion of drug resistant microtubules. Another study also reported microtubule stabilization due to activation of macrophages with IFN- $\gamma$ and LPS stimulation (Binker et al., 2007). Based on this, we decided to investigate the phagocytic 
efficiency of macrophages treated with MSD in the absence of IFN- $\gamma$ priming. It was possible that the lack of inhibition to phagocytosis seen initially was due to overnight priming with IFN- $\gamma$, and removal of this activation might result in inhibition of phagocytosis by macrophages in the presence of MSD. We did not expect a significant decrease in phagocytosis in MSD treated macrophages in the absence of IFN- $\gamma$. We expected that colchicine treatment would inhibit phagocytosis due to colchicine being a microtubule destabilizer. We studied the effect of IFN- $\gamma$ activation on MSD treated macrophages by carrying out pHrodo phagocytosis in parallel, with macrophages that were primed overnight with IFN- $\gamma$, as well as macrophages that were left in media alone for the same duration, followed by MSD treatment and incubation with pHrodo particles. 


\subsubsection{Results}

Figure 5.3.1
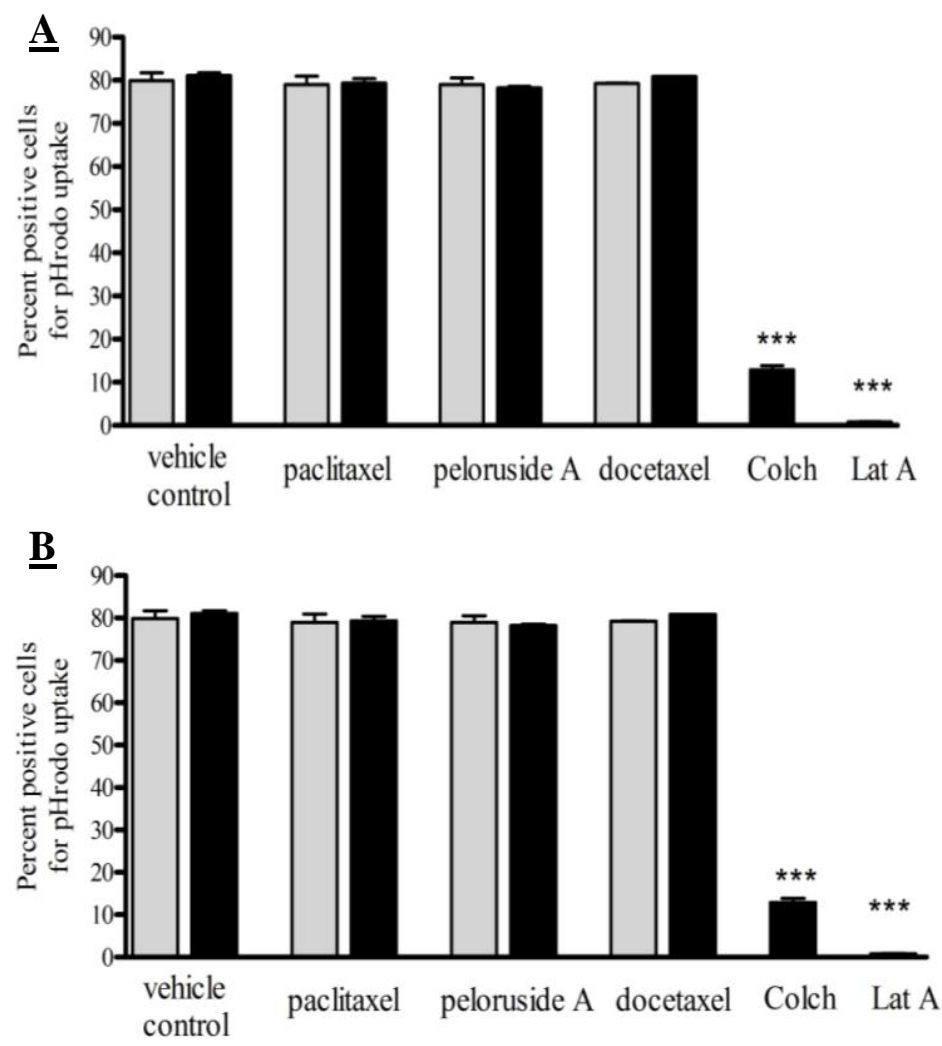

Figure 5.3.1 pHrodo phagocytosis by MSD treated macrophages is not affected, by (A) presence or (B) absence of IFN- $\boldsymbol{\gamma}$ pre-treatment. For both graphs, phagocytosis of pHrodo by MSD treated macrophages is not inhibited as compared to vehicle controls irrespective of IFN- $\gamma$ treatment. There is a significant inhibition to phagocytosis in colchicine treated and latrunculin A treated macrophages as compared to vehicle control, however this inhibition is similar in both the absence and presence of IFN- $\gamma$. For both graphs, gray bars $\square$ represent $0.1 \mu \mathrm{M}$ (low dose) MSD/vehicle control, while black bars represent $1 \mu \mathrm{M}$ (high dose) MSD/vehicle control. Colchicine was used at $10 \mu \mathrm{M}$, corresponding to high dose vehicle control. Bars represent mean \pm SEM from duplicate wells of 1 experiment. $* * * p<0.001$ colchicine vs vehicle control, $* * * p<0.001$ Latrunculin A vs vehicle control, one-way ANOVA with Bonferroni post test. 


\subsubsection{Discussion}

Macrophages pre-treated with IFN- $\gamma$ are activated to respond optimally to bacterial and LPS challenge (Bach et al., 1997; Boehm et al., 1997). However, it was thought that this optimal response due to IFN- $\gamma$ pre-treatment might be masking possible phagocytic inhibition due to MSD treatment. Based on this, we were interested in comparing the effects of MSD on macrophage phagocytosis in the presence and absence of IFN- $\gamma$. Since we were also interested in studying NO and TNF- $\alpha$ production in MSD treated macrophages during phagocytosis, it was important to understand the possible effects of IFN- $\gamma$ pre-treatment as IFN- $\gamma$ has important roles in stimulating production of proinflammatory cytokines IL-12 and TNF- $\alpha$, as well production of NO (Boehm et al., 1997).

We found no difference in the phagocytic abilities of MSD treated macrophages irrespective of IFN- $\gamma$ pre-treatment. Macrophages that were not activated were able to respond to bacterial challenge similarly to activated macrophages. In contrast to the study carried out by Khandani, A. et al. 2007., we found no difference to the ability of colchicine treated macrophages to phagocytose bacteria in the absence or presence of IFN- $\gamma$ pretreatment. The researchers found that IFN- $\gamma$ pre-treatment caused colchicine treated macrophages to phagocytose bacteria similarly to un-treated macrophages, whereas nonactivated macrophages, in the presence of colchicine displayed impaired phagocytosis.

Reasons that could explain why our results did not show a difference with colchicine and IFN- $\gamma$ as compared to colchicine alone could be related to differences in experimental set up. We used $100 \mathrm{U} / \mathrm{ml}$ of IFN- $\gamma$ as compared to $500 \mathrm{U} / \mathrm{ml}$ used in the paper, also the incubation time for phagocytosis was longer in our studies (60 minutes) as opposed to 30 minutes. The higher dose of IFN- $\gamma$ could have increased stability of microtubules and 
created a greater population of drug-resistant microtubules, while the $100 \mathrm{U} / \mathrm{ml}$ dose used by us might not have been sufficient to cause the same degree of stability. The stable microtubules and increased uptake could have led to colchicine treatment having no inhibitory effect in the presence of IFN- $\gamma$. Another consideration is the particle used for testing phagocytic uptake. The presence of live bacteria as compared to polystyrene beads or IgG opsonised sheep red blood cells (SRBC) used in the Khandani, A. et al., study would also have caused different immune responses and activation of different signalling pathways due to differences in size of particles, and receptors involved in uptake (Koval et al., 1998). This could have also caused differences in the ability of macrophages to phagocytose targets. However, both our study and the Khandani, A. et al., 2007 study were carried out for different purposes and the methods were selected to suit different aims, therefore direct comparisons of our differing results is not advisable.

Early electron micrographs have shown the presence of microtubules in regions of actin accumulation around large beads (Reaven et al., 1973), however, there was no specific role for microtubules noted. A later study comparing C3bi mediated and FcR mediated phagocytosis, showed the requirement of microtubules for complement mediated phagocytosis only (Allen et al., 1996), while a contrasting study showed the need for microtubules in FcR mediated phagocytosis (Khandani et al., 2007). Paclitaxel treatment was also seen to affect the efficiency of complement mediated phagocytosis while FcR mediated phagocytosis was less affected (Lewkowicz et al., 2008) Our study looked at bacterial phagocytosis which can occur via FcR if bacteria are coated with antibodies or via complement receptors if bacteria are opsonized with serum protein (Allen et al., 1996). The lack of phagocytic inhibition we saw in our studies in the presence of MSD, both with and 
without IFN- $\gamma$ could be due to the two phagocytic pathways, the complement and the FcR pathway, working together to compensate for any inhibition. It is possible that MSD treatment affected complement pathways to a greater degree; however this may have been masked by the efficiency of the FcR pathway or vice versa. Since paclitaxel and peloruside A have immunomodulatory and immunostimulatory properties (Chan et al., 2000; Crume et $a l ., 2007)$ it could also have been possible that in the absence of IFN- $\gamma$ these drugs may have acted as immune stimulators causing phagocytic activity to be maintained. Paclitaxel mimics LPS, which would have increased the stimulation presented to macrophages to respond to the bacterial challenge. Using cells that constitutively express GFP-labelled tubulin and with the help of confocal techniques, investigating the uptake of pHrodo labelled bacteria in the presence of MSD treatment would be an interesting study. This would allow simultaneous study of the effects of MSD on microtubules and the role of stabilized microtubules in phagocytosis.

Overall we found that absence of IFN- $\gamma$ pre-treatment did not affect phagocytic ability in the presence of MSD treatment. We also found that colchicine treated macrophages exhibited an inhibition in phagocytosis, irrespective of absence or presence of IFN- $\gamma$ pre-treatment. It would be worth investigating the amount of acetylated $\alpha$-tubulin in microtubules during bacterial phagocytosis in MSD and colchicine treated RAW264.7 cells in the presence and absence of IFN- $\gamma$ at differing doses. Levels of acetylated $\alpha$-tubulin indicate level of stabilized microtubules (Schulze et al., 1987), which would allow understanding if the different components such as LPS and IFN- $\gamma$ have roles in stabilizing microtubules and if this stability is maintained in the presence of colchicine. 
Chapter 5: Bacterial phagocytosis by

MSD treated RAW264.7 macrophages.

\subsection{Use of confocal microscopy to visualize pHrodo phagocytosis}

\subsubsection{Introduction}

While flow cytometry allows multiparametric analysis of thousands of cells per minute, resulting in extremely quantitative data, these events cannot be visualized at the cellular level. Confocal microscopy allows live cell imaging to enable visualization of phagocytosis over time. We were able to use the confocal microscope to visualize phagocytosis of pHrodo in RAW264.7 cells, thus complementing our flow cytometry data.

Phagocytosis was visualized in untreated RAW264.7 cells as well as with paclitaxel, Latrunculin A and Colchicine treated RAW264.7 cells. Experiments were set up as described in section 2. Briefly, cells were not stained or IFN- $\gamma$ stimulated. Pre-treatment of cells with the respective drug or vehicle was carried out for 45 minutes following addition of pHrodo. Images were taken 30 minutes after addition of pHrodo and thereafter data was collected every 30 minutes over 4 to 5 hours. We used differential interference contrast (DIC) and red fluorescent channels to collect data, as phase contrast enabled clear visualization of the cell outlines. Additionally, untreated macrophages were stained with blue fluorescent Hoechst 33342 and CFSE or Hoechst alone and incubated with pHrodo to visualize phagocytosis. 


\subsubsection{Results}

Figure 5.4.1
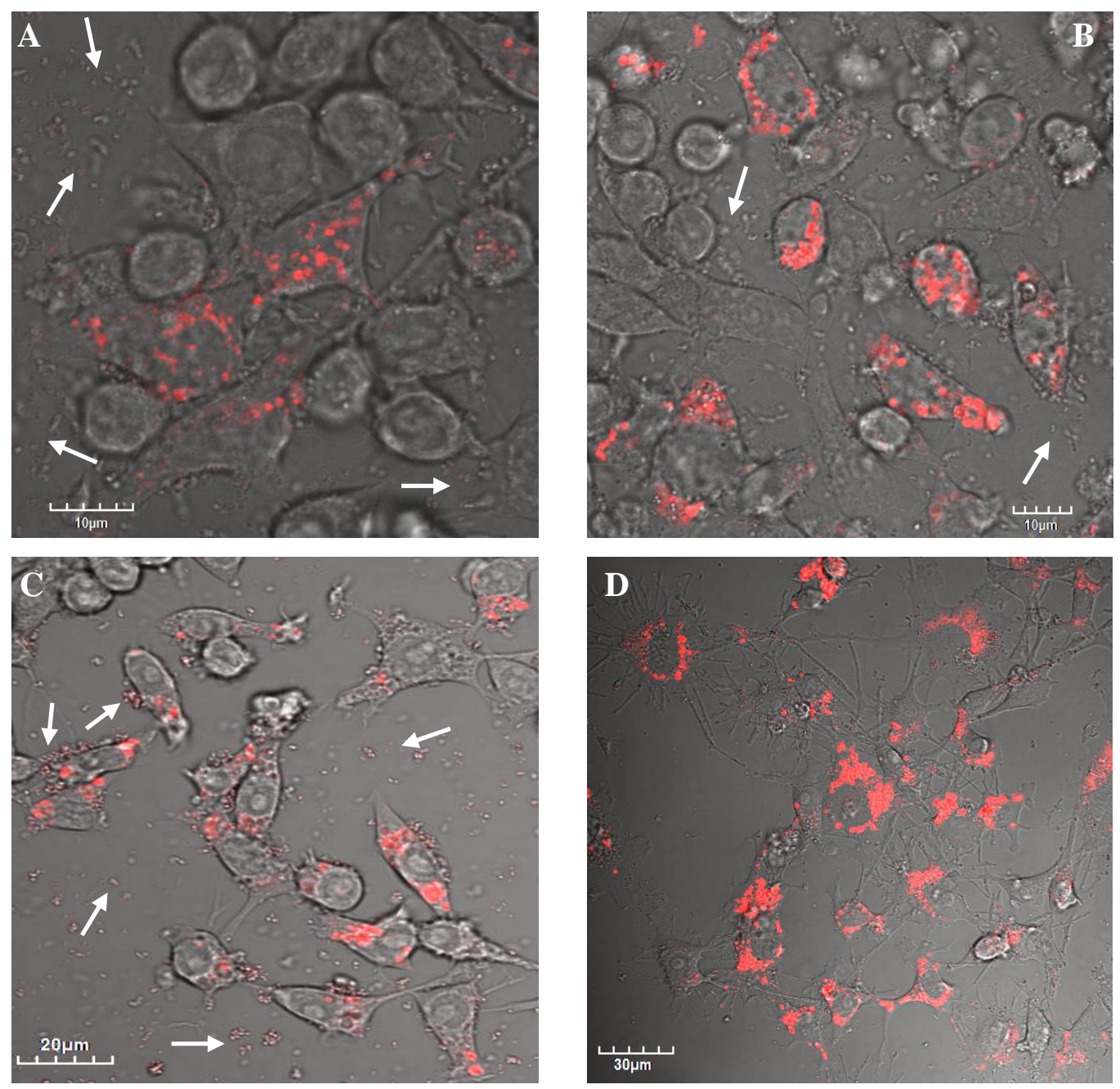

Figure 5.4.1 pHrodo particles fluoresce only when phagocytosed. Untreated RAW264.7 cells incubated with pHrodo particles. Each image is one plane from an XYZ-axis image through a field of view. Internalized pHrodo displays bright red fluorescence while particles outside the cells are non fluorescent $(\rightarrow)$. Representative DIC images with fluorescence overlay. Images collected 8.5 (A, B, C) and 5 hours (D) after addition of pHrodo to the cells. Scale bars represent $10 \mu \mathrm{m},(\mathrm{A}, \mathrm{B}) ; 20 \mu \mathrm{m}$ (C); and $30 \mu \mathrm{m}$ (D). 
Figure 5.4.2 A
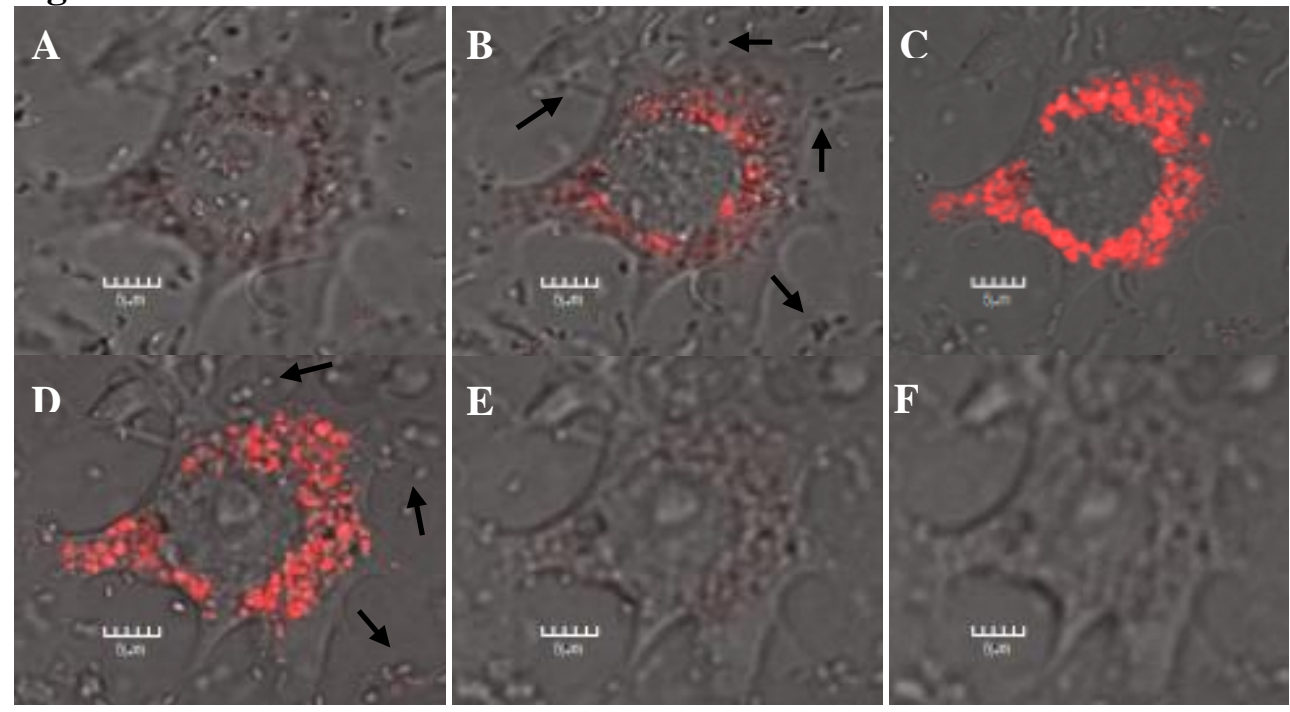

Figure 5.4.2 B
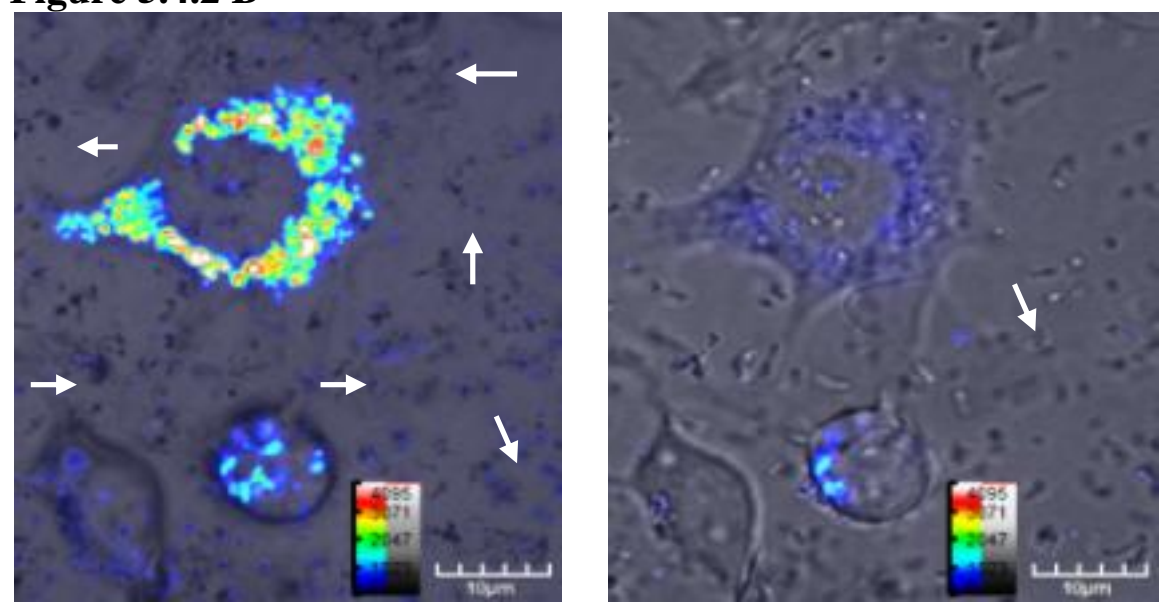

Figure 5.4.2 Phagocytosed pHrodo has a higher intensity of fluorescence as compared to external pHrodo. (A) A series of XYZ-axis images through a field of view, show untreated cells with internalized pHrodo fluorescing brightly, while extra cellular pHrodo is non-fluorescent $(\rightarrow)$ The images show consecutive planes through one field of view, moving up through the cell. Representative DIC images with fluorescence overlay. Scale bars, $5 \mu \mathrm{m}$. (B) A macrophage DIC image with fluorescence intensity overlay. The red fluorescent channel is altered to show intensity. Regions of high intensity are bright blue to red and regions of low intensity are dark blue (white arrows) as seen with the intensity scale bar. Scale bars, $10 \mu \mathrm{m}$. All images collected 8.5 hours after addition of pHrodo to the cells. 
Figure 5.4.3
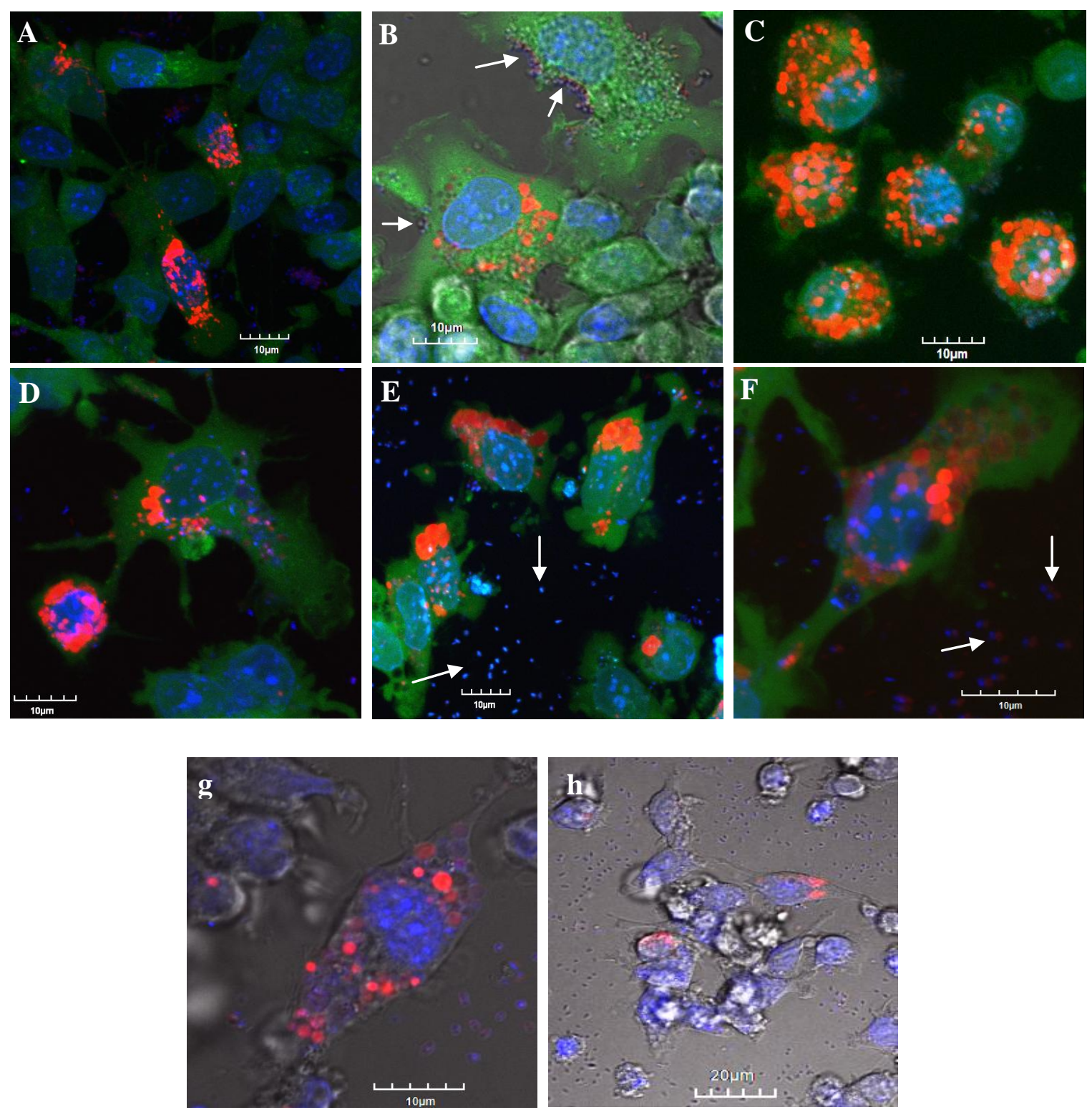

Figure 5.4.3 Series of images showing pHrodo phagocytosis in CFSE and Hoechst stained RAW264.7 cells. (a-f) CFSE and Hoechst stained cells phagocytose pHrodo. (b) Arrows indicate extracellular pHrodo that has adhered to cells, but is non-fluorescent. (e, f) arrows indicate Hoechst stained nuclear material of extracellular pHrodo. (g-h) Hoechst stained cells with phagocytosed pHrodo, extracellular pHrodo is non-fluorescent. Images are one plane from an XYZ-axis image or stacked image of all planes through a field of view. All images were taken 8.5 hours after addition of pHrodo. (a-f) Representative CFSE images with pHrodo and Hoechst overlay $(\mathrm{g}, \mathrm{h})$ Representative DIC images with pHrodo and Hoechst overlay. Scale bars represent $10 \mu \mathrm{m}(\mathrm{a}-\mathrm{g})$, and $20 \mu \mathrm{m}(\mathrm{h})$. 
Chapter 5: Bacterial phagocytosis by MSD treated RAW264.7 macrophages.

Figure 5.4.4

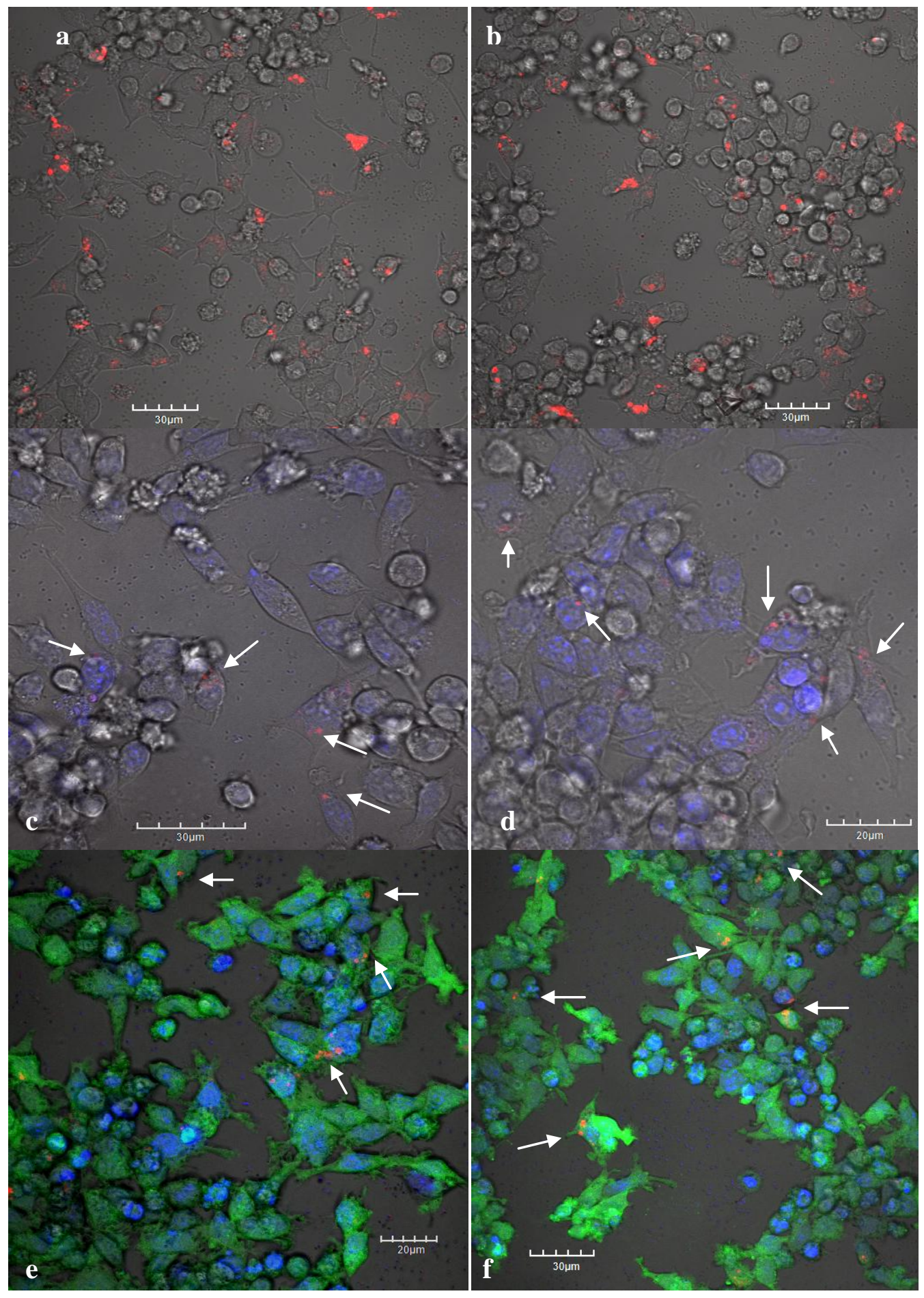


Figure 5.4.4 Unstained cells (A, B) phagocytose more pHrodo as compared to Hoechst (C, D) and CFSE (E, F) stained cells. All images are taken 105 minutes after addition of pHrodo, and unstained cells (A, B) have phagocytosed higher amounts of pHrodo as compared to CFSE (E, F) or Hoechst (C, D) stained cells. Additionally, unstained cells have greater levels of pHrodo per cell as compared to CFSE and Hoechst stained cells. Each image is one plane from an XYZ-axis image through a field of view. Representative DIC images with fluorescence overlay, (A, B). Representative DIC images with pHrodo and Hoechst overlay, (C, D). Representative DIC images with pHrodo and CFSE overlay, (E, F). Scale bars represent $30 \mu \mathrm{m}$ (A, B, C, F), and $20 \mu \mathrm{m}$ (D, E). All images were analyzed as described in the confocal microscopy analysis: Section 2.12 .3 


\section{Figure 5.4.5}
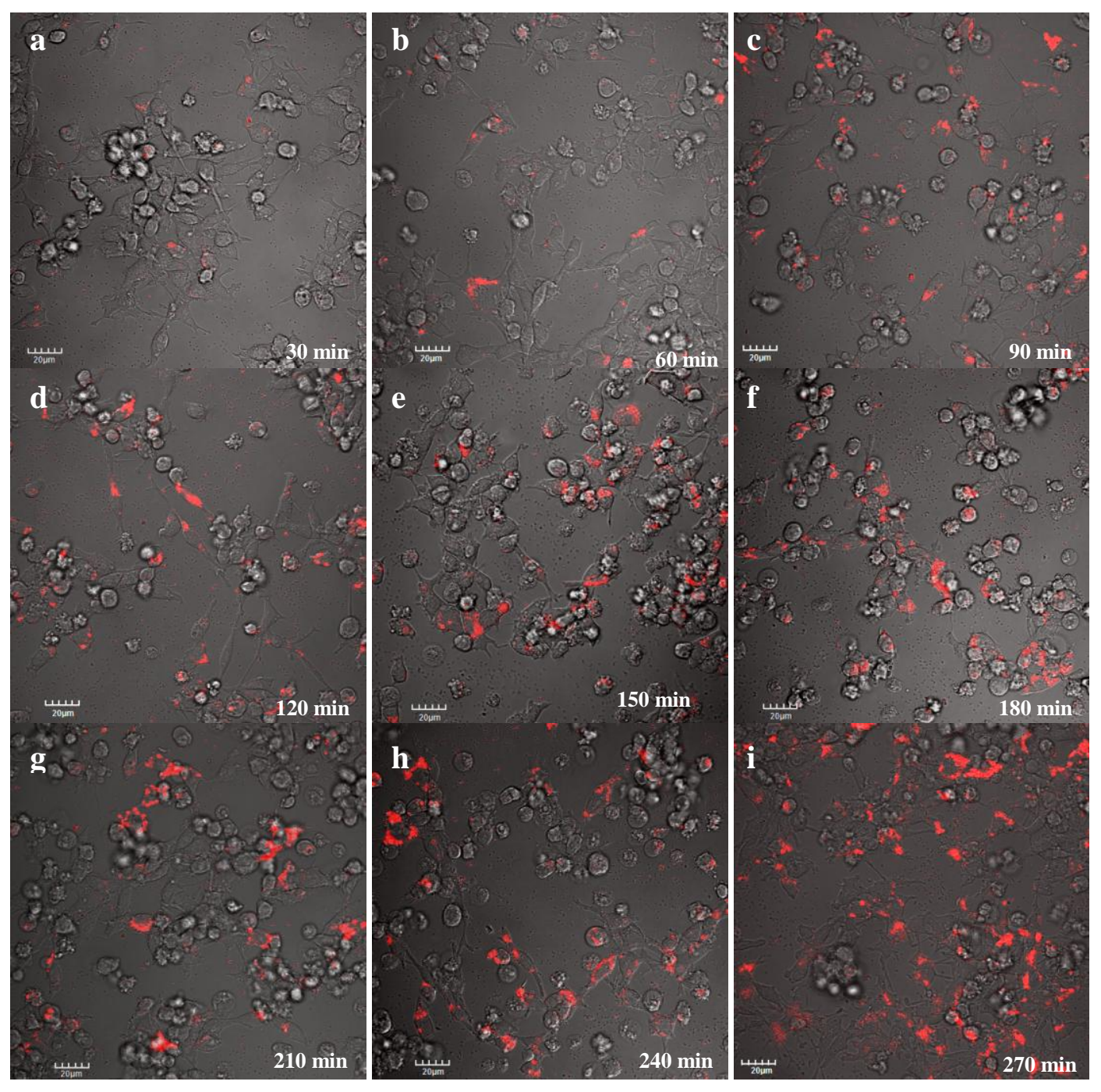

Figure 5.4.5 Time-lapse images showing internalization and acidification of pHrodo

during phagocytosis by RAW264.7 cells. Images show increasing pHrodo fluorescence over time as internalization increases. All images are one plane from an XYZ-axis image through a randomly selected field of view. Images were taken every 30 minutes for 4.5 hours after addition of pHrodo particles to untreated RAW264.7 cells. Representative DIC images with fluorescence overlay. Scale bars represent $20 \mu \mathrm{m}$. 
Chapter 5: Bacterial phagocytosis by MSD treated RAW264.7 macrophages.

Figure 5.4.6

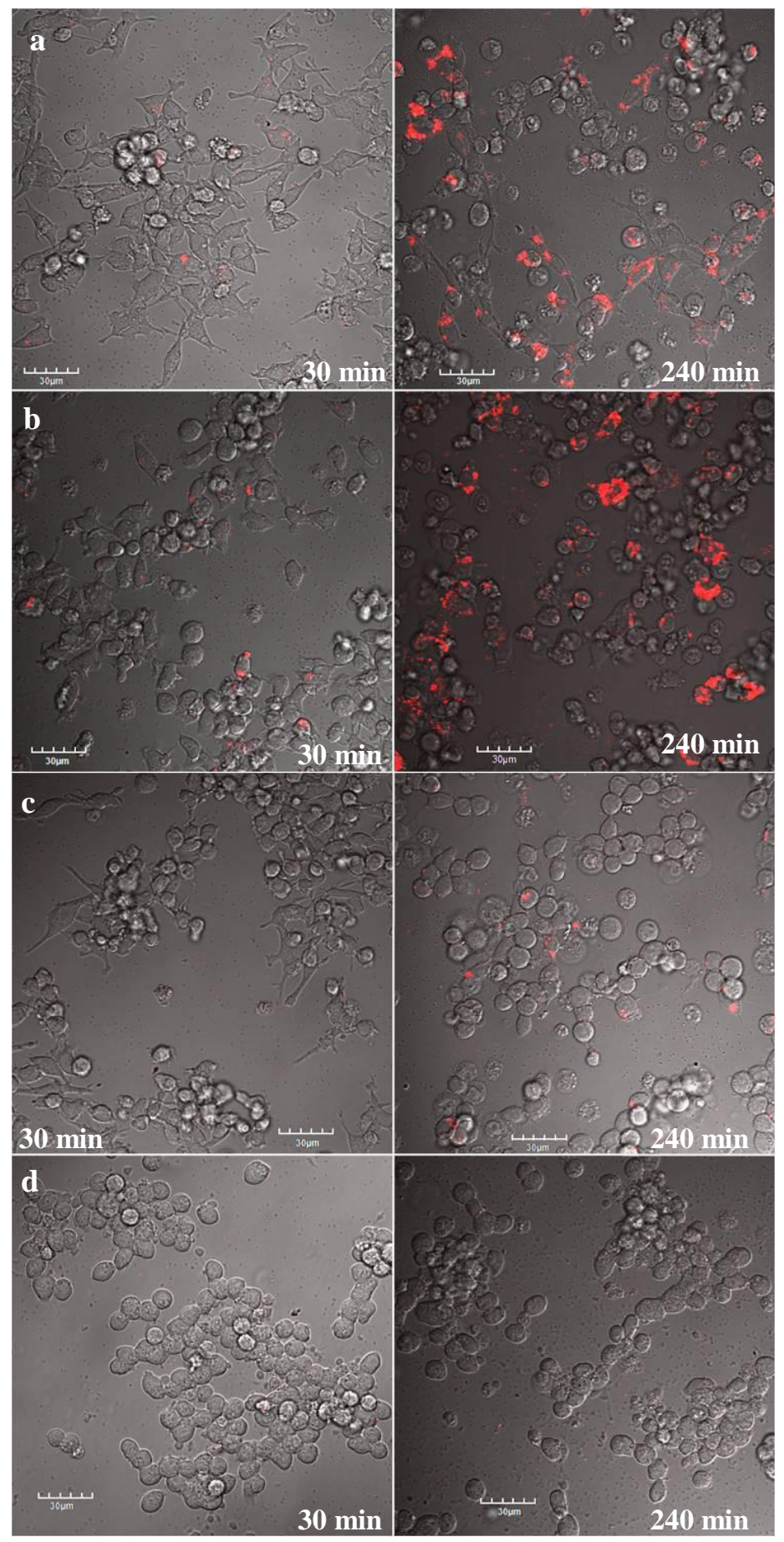

Figure 5.4.6 Phagocytosis of pHrodo accompanied with increase in fluorescence over time in (a) untreated and (b) paclitaxel treated cells, while (c) colchicine and (d) latrunculin A treated cells show reduced or no phagocytosis respectively. Each image is one plane through a field of view, representative of all images at that time point. Time lapse images taken over 4 hours and show level of phagocytosis at 30 minutes and 240 minutes. (Not all images are shown; see appendix $\mathrm{C}$ for all images). Representative DIC images with fluorescence overlay. Scale bars for each image represent $30 \mu \mathrm{m}$. 


\section{Figure 5.4.7}

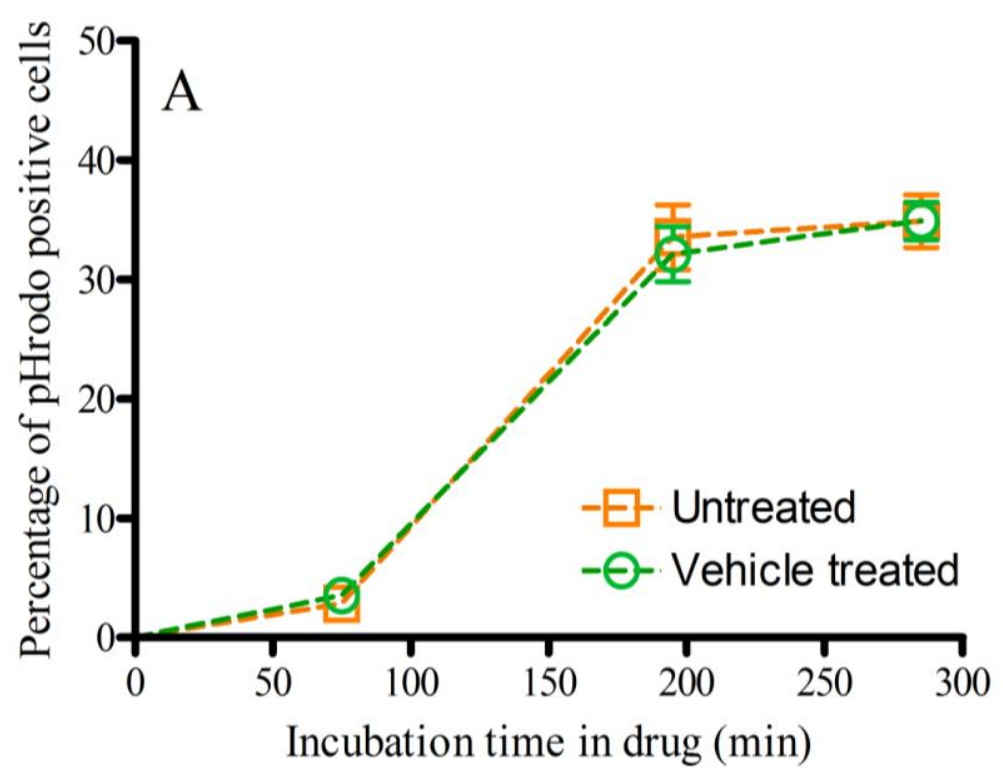

Figure 5.4.7 Vehicle treated macrophages phagocytose pHrodo similarly to untreated macrophages. RAW264.7 cells were left untreated or treated with ethanol as a vehicle control. pHrodo phagocytosis was measured at 75, 195 and 285 minutes after vehicle treatment. Phagocytosis levels between untreated and ethanol treated macrophages are highly comparable. $\mathrm{p}>0.05$, two-way ANOVA with Bonferroni post test. 


\section{Figure 5.4.8}

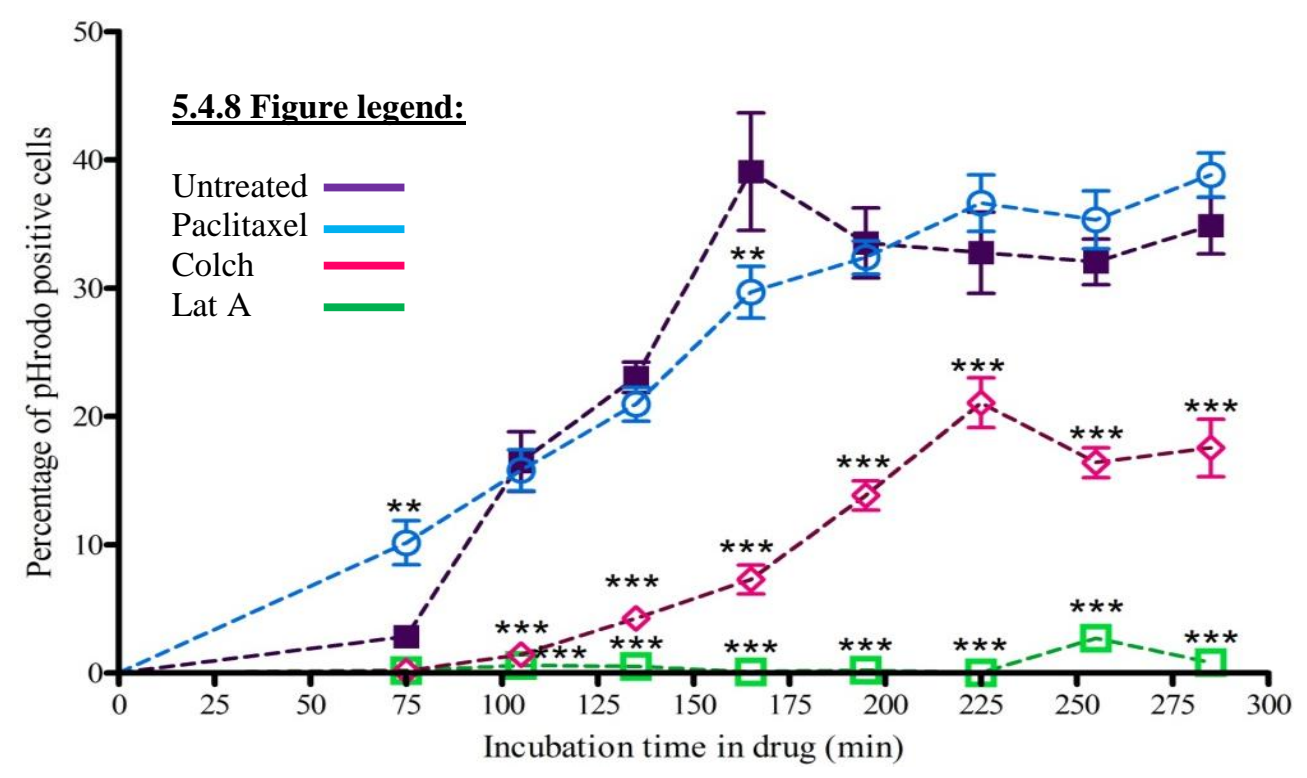

Figure 5.4.8 Colchicine and Latrunculin A treated cells show a significant decrease in phagocytic ability, while paclitaxel treated cells show altered phagocytosis patterns. RAW264.7 cells were left untreated or pre-treated for 45 minutes with $1 \mu \mathrm{M}$ paclitaxel, 1 $\mu \mathrm{M}$ latrunculin A or $10 \mu \mathrm{M}$ colchicine. Phagocytosis was measured at 30 minute intervals after pHrodo addition. As compared to untreated macrophages, latrunculin $\mathrm{A}$ and colchicine treated macrophages show a highly significant decrease in phagocytic ability. While paclitaxel treated macrophages phagocytose pHrodo to similar levels as untreated macrophages, there are significant differences in how fast the phagocytosis occurs. The time taken for phagocytosis to peak before decreasing also differs for untreated and paclitaxel treated macrophages. $* * * \mathrm{p}>0.001$ latrunculin A treated vs untreated, $* * * \mathrm{p}<0.001$ colchicine treated vs untreated, ** $\mathrm{p}<0.01$ paclitaxel vs untreated, two-way ANOVA with Bonferroni post test. 


\section{Figure 5.4.9}

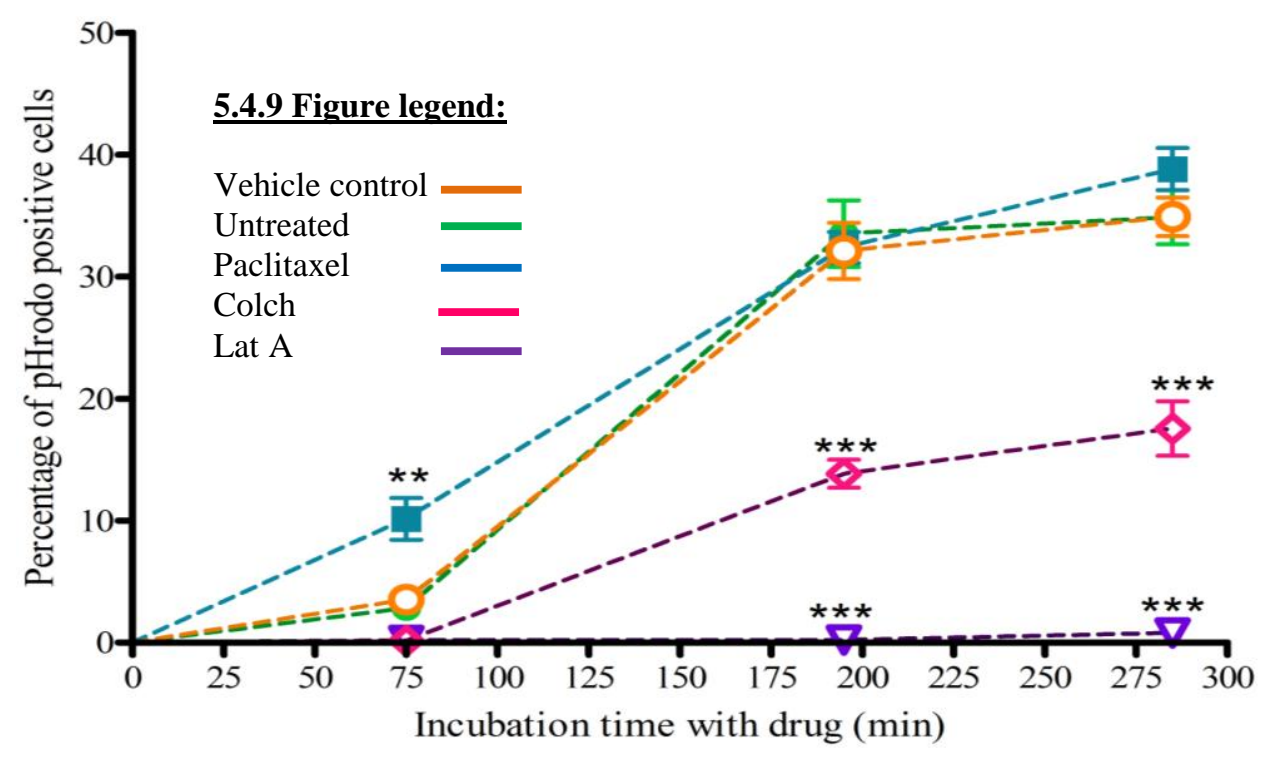

Figure 5.4.9 Colchicine and Latrunculin A treated cells show a significant decrease in phagocytic ability, while paclitaxel treated cells show alterations in initial phagocytic patterns. RAW264.7 cells were left untreated or pre-treated for 45 minutes with $1 \mu \mathrm{M}$ paclitaxel, $1 \mu \mathrm{M}$ latrunculin $\mathrm{A}, 10 \mu \mathrm{M}$ colchicine or equivalent concentration of ethanol as vehicle control. Phagocytosis was measured at 30 minute intervals after pHrodo addition. As compared to vehicle treated macrophages, latrunculin $\mathrm{A}$ and colchicine treated macrophages show a significant decrease in phagocytic ability. While paclitaxel treated macrophages phagocytose pHrodo to similar levels as untreated and vehicle treated macrophages, there is a significant difference in how fast the phagocytosis occurs. $* * * \mathrm{p}>0.001$ latrunculin A treated vs vehicle control, *** $\mathrm{p}<0.001$ colchicine treated vs vehicle control, **p<0.01 paclitaxel vs vehicle control, two-way ANOVA with Bonferroni post test. 


\subsubsection{Discussion}

Using the confocal microscope to visualize cells was extremely beneficial. Firstly, it enabled us to ensure that the pHrodo dye was working according to manufacturer's specifications, and that we were observing phagocytic events accurately. It allowed excellent visual confirmation that fluorescence was only with internalized pHrodo particles. The use of confocal imaging provided us with a different approach via which we could confirm results from flow cytometry, thus validating both our techniques as the results obtained from these techniques were comparable.

When initially optimizing imaging procedures, similarly as with flow cytometry assays, we stained cells with CFSE before addition of pHrodo. However, we found that cells labelled with CFSE alone, Hoechst alone or Hoechst and CFSE together took longer to phagocytose pHrodo as compared to unstained cells. In addition, these stained cells also took up lower concentrations of pHrodo as compared to unstained cells. It is known that CFSE at high concentrations is toxic to cells (Parish et al., 2001). The staining protocol used for confocal microscopy experiments was consistent with flow cytometry staining protocols as described in section 2. However, one factor that could have contributed towards the CFSE toxicity seen in confocal experiments was the day on which the cells were stained. With confocal assays, the cells were seeded at low concentrations and allowed to proliferate, and CFSE staining was carried out on adherent cells on the day of the experiment. This meant that dye quenching and washing steps were carried out to prevent cells from detaching. In contrast, with the flow cytometry assays, the cells were stained while in suspension and washed at least 3 times before being allowed to adhere overnight. This was followed by replacing old media with new media on the day of the 
experiment. The insufficient washing of adherent cells and the fact that staining was carried out on the experimental day could have increased dye concentrations and caused toxicity to the cells, with the result that pHrodo phagocytosis was slower. However staining cells before seeding was not suitable as CFSE dye is used to measure proliferation and gets divided in daughter cells, which means that our staining would have not been uniform across the cell monolayers. We did however notice that CFSE stained cells, if incubated with pHrodo overnight, were able to phagocytose pHrodo similarly to overnight incubated unstained cells. This indicated that phagocytosis was not impaired but took longer due to the presence of dye. An improvement to the protocol used, would be to seed cells and allow monolayer proliferation at least 3 days in advance, followed by cell staining on the day previous to the experimental day to allow sufficient dye efflux.

Nevertheless, the use of unstained cells was sufficient for our aims. Analysis of the confocal assay data indicates that phagocytosis increases until 175 to 200 minutes. With flow cytometry assays we collected data after a 120 minute incubation, and found no inhibition to phagocytosis in the presence of paclitaxel. However, with increasing time, paclitaxel treated macrophages have phagocytic levels comparable to those of untreated or vehicle treated macrophages. This suggests that our flow cytometry data is reliable and the time frames chosen were sufficient.

Since phagocytosis in vehicle treated and untreated macrophages are extremely comparable, it was possible to compare phagocytosis in drug treated macrophages to untreated and vehicle treated macrophages separately (Fig 5.4.5 and Fig 5.4.6). It was necessary to collect data from all treatments on the same day to be able to compare results, 
therefore data was collected every 30 minutes for 4 hours in untreated and drug treated macrophages, however with the vehicle treated cells, data was collected at 75, 195 and 285 minutes after vehicle treatment. Due to this, when analyzing data, comparisons made to vehicle treated cells are made at the 75, 195 and 285 minute time point, but when comparing data to untreated cells, all time points over the collection period are analyzed. Comparison of phagocytosis over short time frames for paclitaxel and untreated macrophages shows interesting results. The data suggest that overall phagocytosis is not impaired in paclitaxel treated macrophages, however, the kinetics of uptake may be different. From figure 5.4.5, we see that initial rate of phagocytosis is faster in paclitaxel treated macrophages. It is also interesting that as compared to paclitaxel treated macrophages, untreated macrophages reach their peak phagocytic capacity earlier and then decline phagocytosis to maintain a steady rate of uptake. In contrast, in the paclitaxel treated macrophages the phagocytic peak occurs later but amounts of pHrodo phagocytosed are comparable to untreated macrophages. This difference in kinetics could be due to the stabilizing effect of paclitaxel on microtubules, as well as its LPS-mimicry which could be providing additional stimulation to macrophages. The trafficking of vesicles via motor proteins on stabilized microtubules is still possible (Mizuno et al., 1994; Apodaca, 2001), indicating that phagocytosis might be unaffected if populations of microtubules are stabilized.

Another explanation could be related to paclitaxel's LPS mimicry. In the presence of paclitaxel, there would be additional stimulation of macrophages to respond to LPS and this could explain the initial increase in rate of phagocytosis. Furthermore, LPS interacts with microtubules causing their stabilization (Ding et al., 1992; Allen et al., 1997b). LPS 
stimulation also causes changes in microtubule composition (Allen et al., 1997a). Phagosome maturation occurs along the endocytic pathway (Harrison et al., 2002) and movement of the phagosome is microtubule dependent. It has been suggested that attachment of phagosomes to microtubules occurs via MAPs (Blocker et al., 1996). The fact that LPS can alter microtubule stability and composition could lead to increased phagosome binding and these factors combined with paclitaxel stimulation could explain lack of inhibition to phagocytosis in the presence of paclitaxel as well as the initial increase in phagocytic rate in paclitaxel treated macrophages.

When compared to vehicle treated cells, the difference in rate of phagocytosis in paclitaxel treated cells is not as evident due to the longer time intervals during imaging. However, the data shows an initial increase in phagocytosis in paclitaxel treated macrophages, similar to the increase seen when comparing paclitaxel treated macrophages to untreated macrophages. Imaging with longer intervals leads to masking of the phagocytic saturation time points, suggesting that overall, phagocytosis is not affected with paclitaxel, or vehicle treatment, but the efficiency and kinetics differ in paclitaxel treated macrophages.

Colchicine treated macrophages displayed impaired phagocytosis. While these cells did phagocytose pHrodo, the rate of phagocytosis was significantly slower and significantly lower amounts of pHrodo were phagocytosed. This was similar to results seen with flow cytometry. The data suggest that phagocytosis is significantly impaired in the presence of microtubule destabilizers, such as colchicine, as compared to little or no inhibition in the presence of microtubule stabilizers. This once more endorses the fact that the presence of stable microtubules is not as detrimental to macrophage function as the presence of 
destabilized microtubules. Latrunculin A treatement completely blocked phagocytosis as expected (Oliveira et al., 1996) and affirms the greater need for actin during initial phagocytosis.

Data from these assays support the use of pHrodo labelled E.coli in allowing study of phagocytic mechanisms where intracellular and extracellular events needed to be defined. We found that the results from confocal studies backed up the results from the flow cytometry studies and in addition allowed visualization of phagocytosis. It would be interesting to study the kinetics of phagocytosis as MSD could affect the rate at which phagocytosis occurs. Furthermore, visualizing phagocytosis in peloruside treated macrophages similarly as with colchicine and paclitaxel treated macrophages would also be extremely beneficial to understanding any differences in phagocytic efficiency. These experiments also support the fact that studying phagocytosis for longer time points is more informative. Phagocytosis occurs within two hours, as seen from flow cytometry data, however studying the rate of phagocytosis and time course over which MSD treated macrophages maintain the rate of phagocytosis would also be useful.

Our results taken together suggest that at the tested doses, treatment with paclitaxel, docetaxel and peloruside A does not inhibit bacterial phagocytosis. However, zampanolide treatment significantly affects phagocytosis at the doses tested. We also found that mycothiazole and ixabepilone do not significantly inhibit bacteria phagocytosis. It is possible that if drug treatments were carried out for longer time periods that we would see an inhibition of phagocytosis, with the other tested MSD, however the methods we used were comparable to previous studies. This allows us to draw reasonable conclusions 
regarding phagocytosis ability and the effects of MSD treatment. We also found that IFN- $\gamma$ activation had no effect on enhancing or inhibiting phagocytosis in the presence of MSD or colchicine treatment. 
Chapter 6: Bacterial killing by

MSD treated RAW264.7 macrophages.

\section{CHAPTER 6: BACTERIAL KILLING BY MSD TREATED RAW264.7 MACROPHAGES}

\subsection{Introduction}

Macrophage phagocytosis involves binding, ingestion and killing of the pathogen. As discussed in previous sections, while the initial phagocytic events are actin dependent, (Aderem et al., 1999; Al-Haddad et al., 2001; Underhill et al., 2002) evidence suggests that the latter phagocytic events, such as transport from the cell periphery towards the nuclear region, are microtubule dependent (Toyohara et al., 1989; Desjardins et al., 1994b; Blocker et al., 1996) Studies have also shown that newly formed phagosomes require dynamic microtubules for slow movements and that bi-directional movement of phagosomes along the microtubules requires the microtubule molecular motors kinesin and dynein (Blocker et al., 1997; Blocker et al., 1998).

We had studied the effect of MSD on initial phagocytic events, but since later phagocytic events are more microtubule dependent, we decided to investigate the effect of MSD on macrophage killing ability. In particular, we looked at the ability of MSD treated macrophages to exert bactericidal effects. Since it is known that paclitaxel exhibits LPS mimicry (Ding et al., 1990b; Bogdan et al., 1992) it was thought that paclitaxel treated macrophages may exhibit increases in bacterial killing by increasing macrophage stimulation. Additionally, to date there are no studies that have looked at the effects of docetaxel or peloruside A on macrophage killing, making this study interesting in terms of development of these drugs for clinical use. Treatment with MSD could also alter phagosome trafficking and thus alter cytokine secretion since phagosomes play a role in secretion of cytokines (Murray et al., 2005). 
Macrophage bactericidal activity in the presence of MSD was studied using a gentamicin survival assay as described in the methods. In brief, this assay works on the principle of antibiotic susceptibility of extracellular bacteria, while intracellular bacteria are protected from the antibiotic (Fig 6.1.1) (Vaudaux et al., 1979; Elsinghorst, 1994; Laroux et al., 2005). To carry out these assays macrophages were incubated with bacteria for phagocytosis to occur; following which $100 \mu \mathrm{g} / \mathrm{ml}$ gentamicin was used to kill extracellular bacteria. Cells were then washed in PBS and lysed with Triton X-100 to recover intracellular bacteria. We also included control wells, where cells were incubated with bacteria, but not treated with gentamicin. Instead, after the incubation for phagocytosis these cells were immediately lysed with Triton X-100 to recover intracellular and extracellular bacteria. All the samples were then diluted onto Luria Bertoni (LB) agar plates to allow overnight colony growth, after which colony forming units (CFU) were counted. The control wells enabled us to have an idea of the total bacterial counts as compared with gentamicin treated samples that showed only internalized bacteria. The CFU counted were bacteria that were phagocytosed and survived inside the macrophages. In addition, there were cells without MSD treatment that were incubated with bacteria. These macrophages were gentamicin treated and lysed similarly to other samples to give CFU counts. Thus comparing the non drug treated CFU counts to MSD treated CFU counts enabled us to assess macrophage bactericidal ability as high CFU counts equate to impaired killing, while low CFU counts equate to enhanced killing. 


\section{Figure 6.1.1}

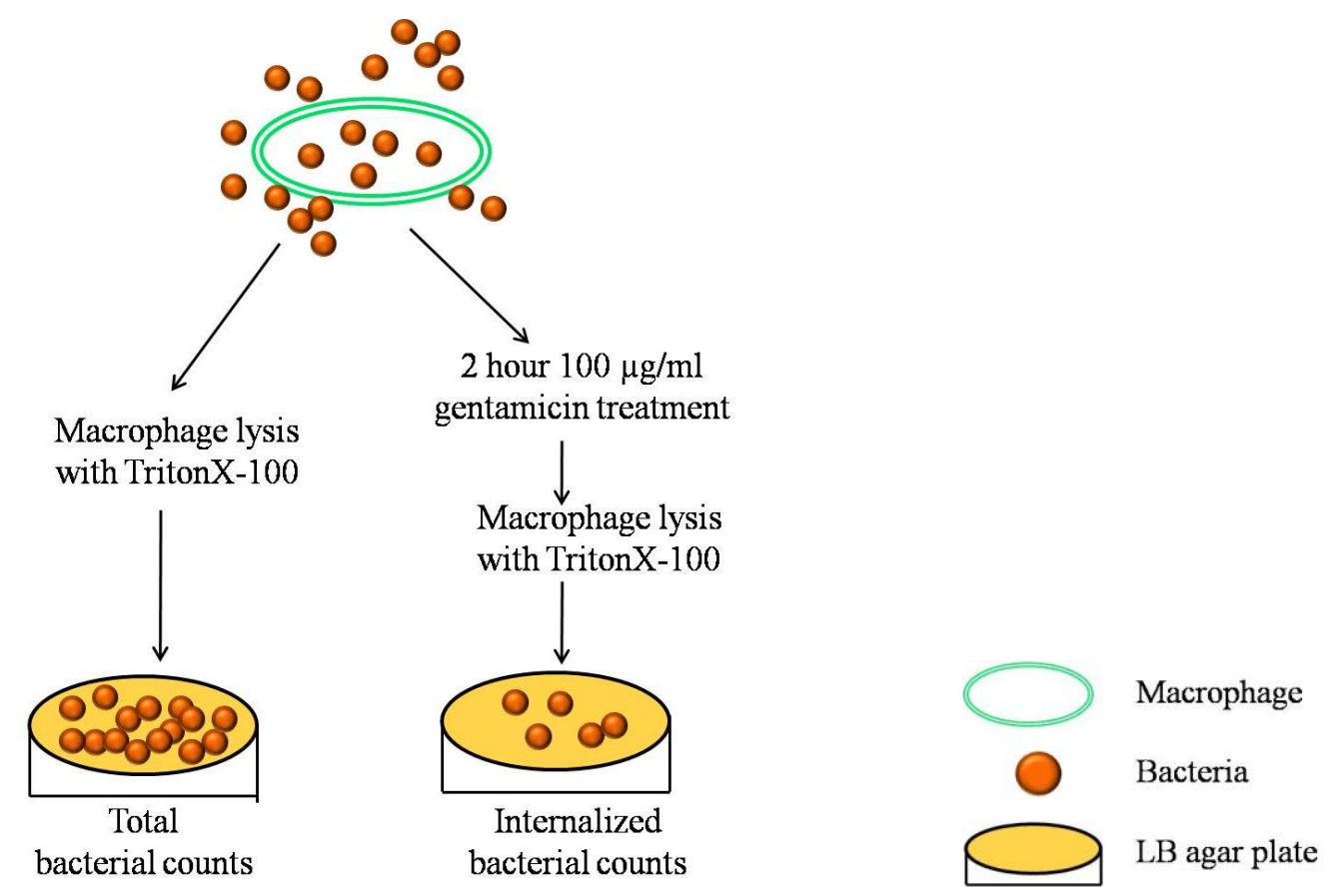

Figure 6.1.1 Plating of bacteria after gentamicin treatment enables determination of macrophage bactericidal activity. 1 hour infection of macrophages allows phagocytosis to occur. Treatment with $100 \mu \mathrm{g} / \mathrm{ml}$ gentamicin kills extracellular bacteria, but intracellular bacteria are protected. Lysed macrophages are plated on LB agar before internal killing occurs. Comparison of bacterial CFU in MSD treated or untreated macrophages enables determination of enhanced or impaired bactericidal activity. 


\subsection{Results}

\subsubsection{Phagocytosis of E.coli in RAW264.7 macrophages.}

Initially we tested the uptake of differing concentrations of E.coli with untreated RAW264.7 macrophages to ensure assays were carried out at the optimal time-frame and bacterial concentration required for phagocytosis to occur. Bacterial infections are normally set up using bacteria: macrophage infection ratio called the multiplicity of infection (MOI) (O'Riordan et al., 2002; Sukumaran et al., 2003). Since these studies have reported using MOI values from 0.1:1 up to 150:1, we optimized bacterial MOI for phagocytosis (Fig 6.2.1). We tested bacterial phagocytosis in RAW264.7 macrophages as well as in J774.2 murine macrophages to compare internalization efficiency in both these cell lines (Fig 6.2.2). Internalization of bacteria was confirmed using cytospins (Fig 6.2.3), which were stained for visualization via giemsa staining as described in the method section 2.8. We also tested if opsonisation of bacteria or IFN- $\gamma$ pre-treatment of macrophages was required for optimal phagocytic efficiency (Fig 6.2.4). 
Figure 6.2.1
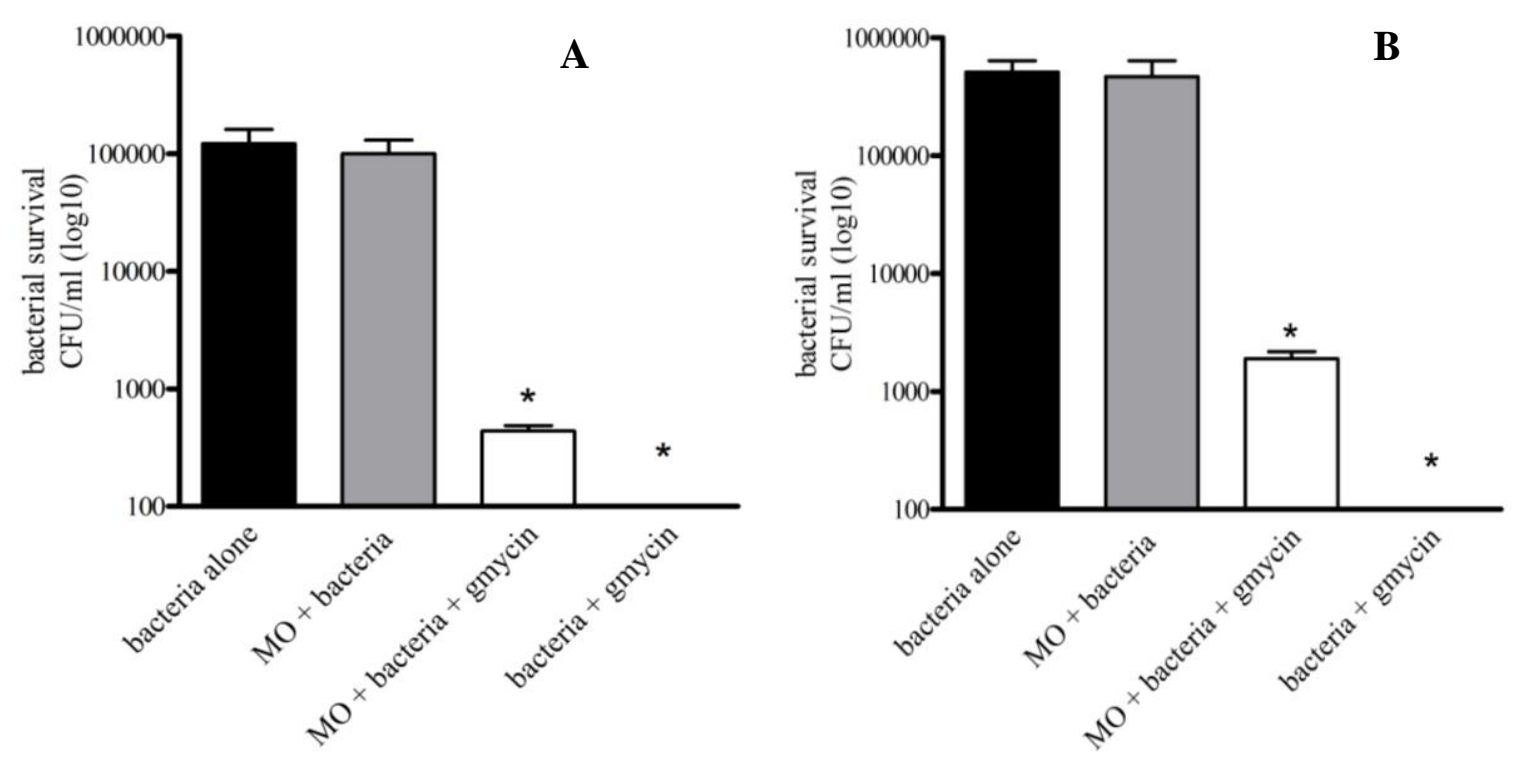

Figure 6.2.1 Phagocytosis of bacteria in RAW264.7 macrophages at varying MOI.

Cells were seeded at $2 \times 10^{6} / \mathrm{ml}$ and infected with bacteria at an MOI of (A) 10:1 bacteria: macrophage or (B) 25:1 bacteria: macrophage. There was no significant difference between the amount of bacteria internalized at either MOI 10 or MOI 25, when compared to each other. There was a significant reduction in bacterial colony forming units $/ \mathrm{ml}(\mathrm{CFU} / \mathrm{ml})$ after gentamicin treatment. Bars represent mean survival of bacteria as $\mathrm{CFU} / \mathrm{ml} \pm \mathrm{SEM}$. Bacteria alone and macrophage $(\mathrm{MO})+$ bacteria represent total live bacteria, while bacteria with macrophages and gentamicin shows internal live bacteria only. Graph is representative of at least 3 experiments. ${ }^{*} \mathrm{p}<0.05$, one-way ANOVA with Bonferroni post test. 
Figure 6.2.2
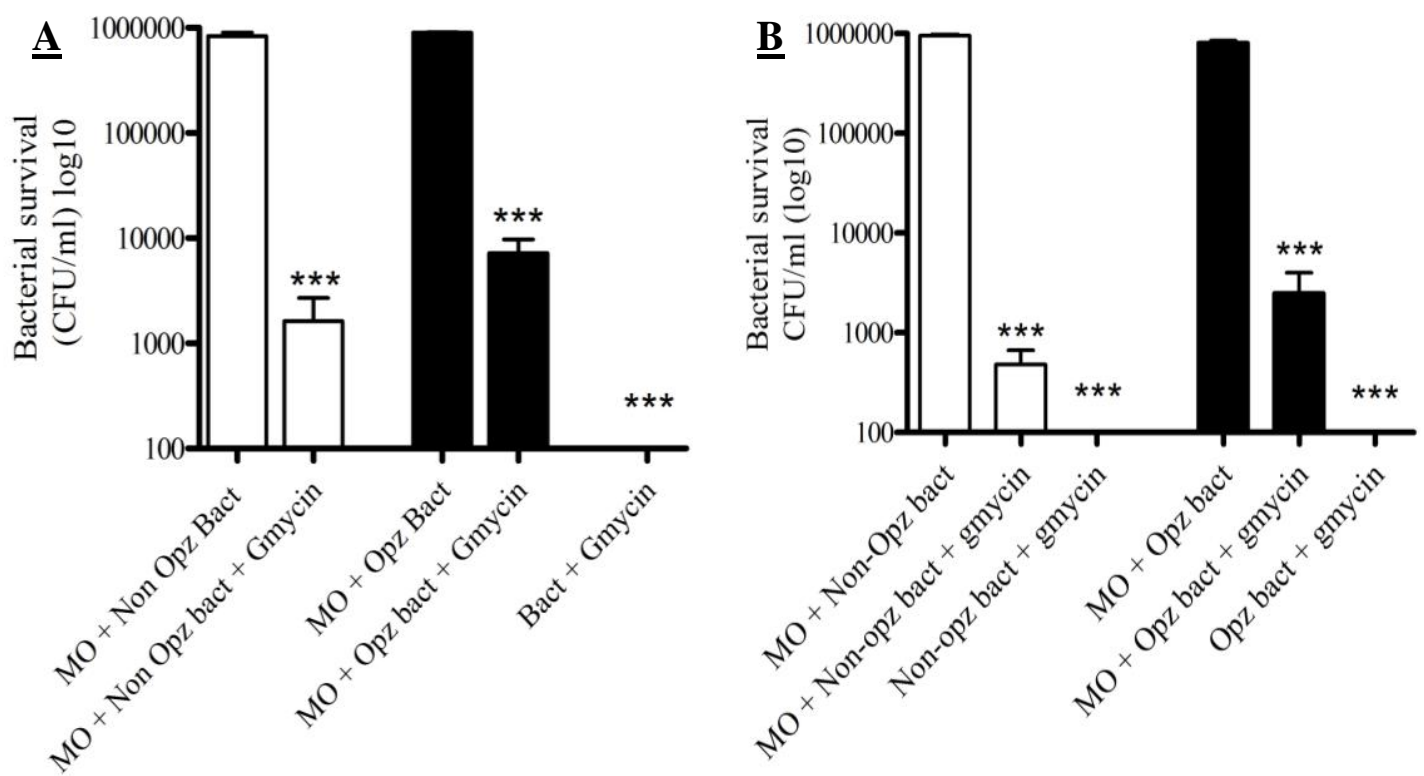

Figure 6.2.2 Effect of opsonisation on phagocytosis of bacteria at MOI 25:1 in (A) J774.2 and (B) RAW264.7 macrophages. Cells were seeded at $2 \times 10^{6} / \mathrm{ml}$ and infected with bacteria at an MOI of 25:1. There was no significant difference between the levels of bacteria internalized either with or without opsonisation in either cell line. There was a significant difference in survival of bacteria after gentamicin treatment in both cell lines. For both graphs, bars represent mean survival of bacteria as colony forming units/ml $(\mathrm{CFU} / \mathrm{ml}) \pm \mathrm{SEM}$. Open bars $\square$ represent samples with non-opsonized bacteria, while dark bars represent samples with opsonized bacteria. Bact: bacteria, MO: macrophage, Gmycin: gentamicin, Opz: opsonized, N-Opz: non-opsonized. Graph is representative of at least 3 experiments. For both graphs: $* * * p<0.001 \mathrm{MO}+$ non-opsonized bacteria and gentamicin vs $\mathrm{MO}+$ non-opsonized bacteria, ***p<0.001 MO + opsonized bacteria and gentamicin vs $\mathrm{MO}+$ opsonized bacteria, $* * * \mathrm{p}<0.001$ bacteria + gentamicin vs $\mathrm{MO}+$ nonopsonized bacteria, ${ }^{* *} \mathrm{p}<0.001$ bacteria + gentamicin vs MO opsonized bacteria, one-way ANOVA with Bonferroni post test. 
Figure 6.2.3
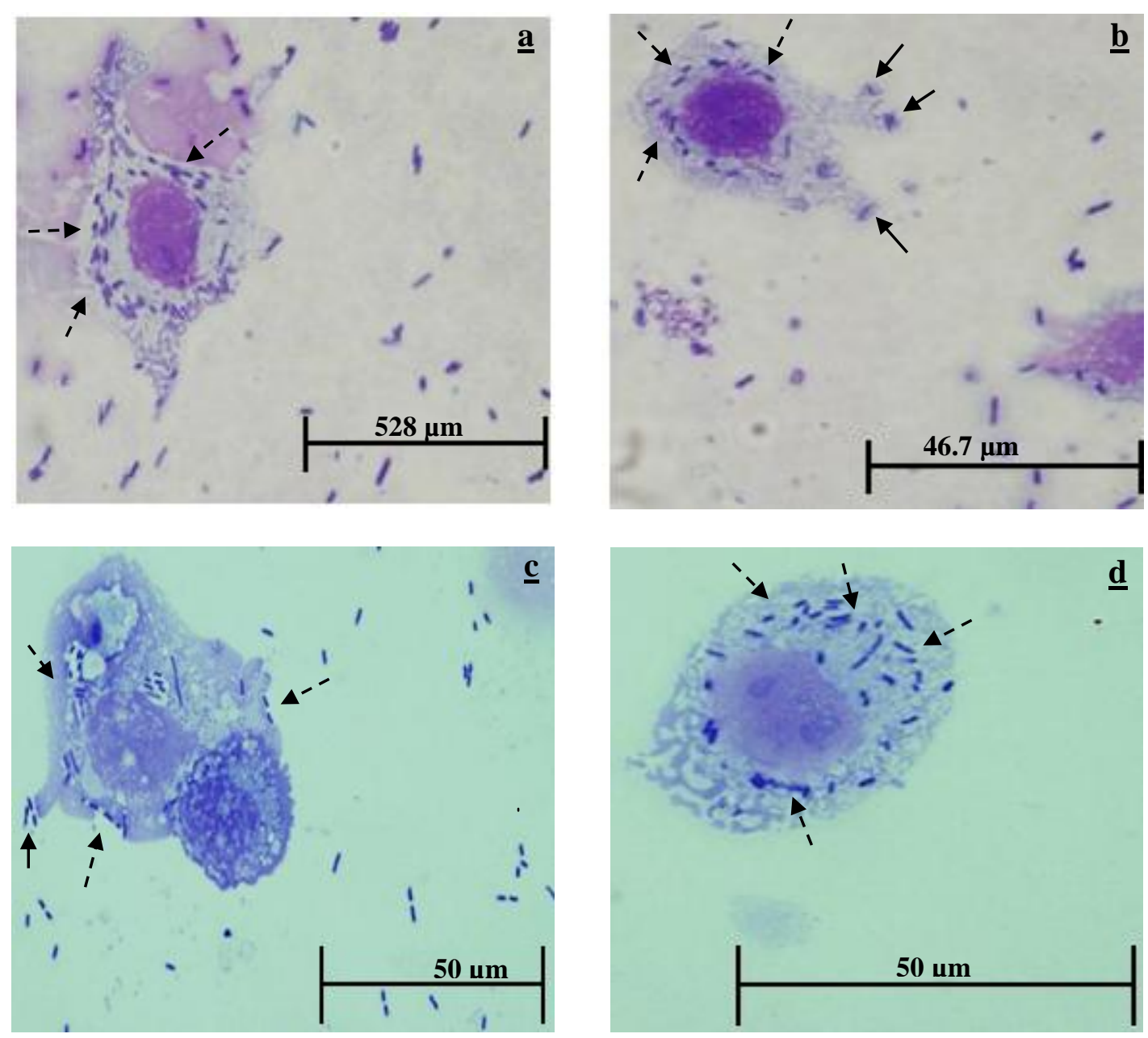

Figure 6.2.3 Confirmation of E.coli internalization by RAW264.7 and J774.2 macrophages. Cells were seeded at $2 \times 10^{6} / \mathrm{ml}$ and incubated with MOI 25 bacteria for 1 hour, followed by 2 hours incubation with $100 \mu \mathrm{g} / \mathrm{ml}$ gentamicin. (a, b) RAW264.7 cells with internalized bacteria (dark blue rods), shown with $\rightarrow$ arrows. $\rightarrow$ arrows indicate macrophage pseudopodia in the process of internalization of bacteria. (c, d) J774.2 cells with internalized bacteria (dark blue rods), shown with $\rightarrow$ arrows. $\rightarrow$ arrows indicate macrophage pseudopodia in the process of internalization of bacteria. Cytospin images taken under bright field 100X magnification. 
Figure 6.2.4

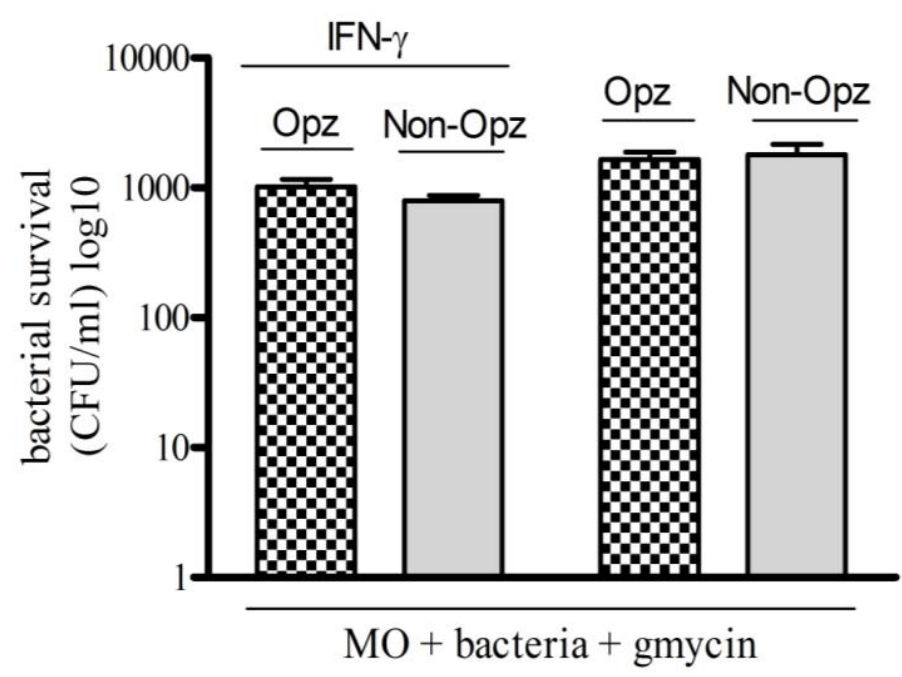

Figure 6.2.4 RAW264.7 macrophages phagocytose similar levels of bacteria irrespective of IFN- $\gamma$ pre-treatment or bacterial opsonisation. Cells were pre-treated with $100 \mathrm{U} / \mathrm{ml}$ IFN- $\gamma$, seeded and incubated with bacteria as described in methods. There was no significant difference in the survival of bacteria either with or without opsonisation of bacteria. Macrophage pre-treatment with IFN- $\gamma$ did not significantly alter survival of bacterial. Bars represent mean survival of bacteria as $\mathrm{CFU} / \mathrm{ml} \pm \mathrm{SEM}$. MO: macrophage, Gmycin: gentamicin, Opz: opsonized, N-Opz: non-opsonized. Graph is representative of at least 3 experiments. $\mathrm{p}>0.05$, one-way ANOVA with Bonferroni post test. 


\subsubsection{Bacterial phagocytosis and killing in MSD treated RAW264.7 macrophages}

Macrophages were seeded and stimulated overnight with $100 \mathrm{U} / \mathrm{ml}$ of IFN- $\gamma$, as described in the methods. Following this, a 45 minute drug treatment was carried out with low $(0.1 \mu \mathrm{M})$ or high $(1 \mu \mathrm{M})$ doses of MSD or the equivalent vehicle control. Bacteria were then added to the cells at MOI of $25: 1$. Drug concentration was maintained at all times following addition of bacteria. Un-treated macrophages infected with bacteria and bacteria alone were also included as controls. We treated macrophages with paclitaxel, docetaxel and peloruside A (Fig 6.2.5). Additionally we also investigated the effects of zampanolide, ixabepilone and mycothiazole (Fig 6.2.6). As with pHrodo studies, we included Latrunculin A as a positive control for these experiments. We expected inhibition of phagocytosis in macrophages treated with Latrunculin A and therefore no survival of colonies after gentamicin treatment.

Results are expressed as mean survival of bacteria as colony forming units per millilitre (CFU/ml). Data from these studies fell into a high CFU/ml (control CFU > 1000 $\mathrm{CFU} / \mathrm{ml}$ ) or low $\mathrm{CFU} / \mathrm{ml}$ (control $\mathrm{CFU}<1000 \mathrm{CFU} / \mathrm{ml}$ ) categories, which is noted in figure legends. Compared to vehicle controls, treatment of macrophages with paclitaxel, peloruside A or docetaxel at high or low doses, did not significantly affect the macrophage killing ability when bacterial colony counts were high. However when bacterial counts were low, there was a significant difference in the killing ability of low dose paclitaxel treated macrophages. Compared to vehicle treated cells, treatment with zampanolide, mycothiazole, or ixabepilone did not significantly alter bactericidal activity of macrophages. 
Figure 6.2.5
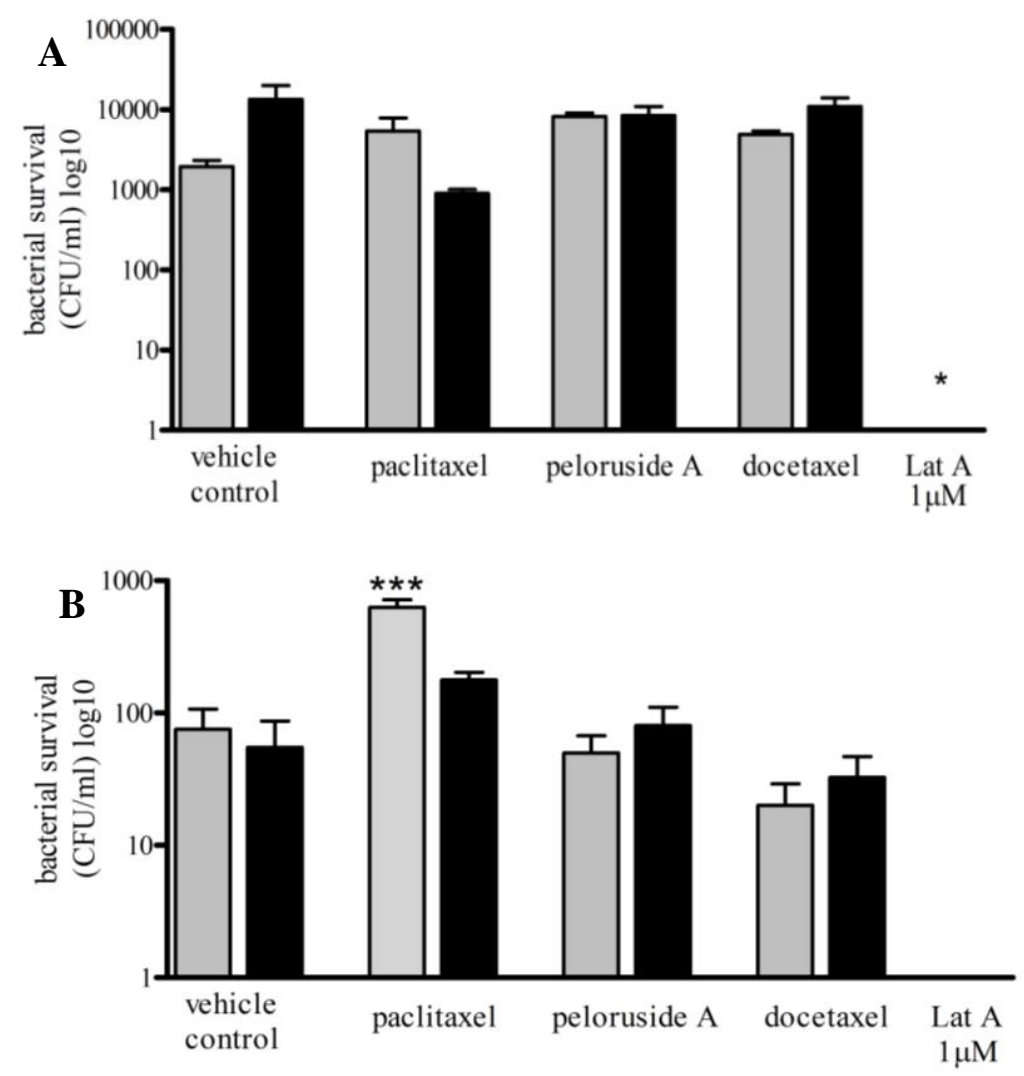

Figure 6.2.5 Bacterial survival in RAW264.7 macrophages treated with high and low dose MSD. (A) Bacterial survival was in the high CFU/ml range. Compared to vehicle controls, neither paclitaxel, peloruside A nor docetaxel significantly alters the killing ability of macrophages. (B) Bacterial survival was in the low CFU/ml range. Compared to vehicle controls, only low dose paclitaxel significantly impaired killing ability of macrophages. In both experiments, all samples are gentamicin treated. Bars represent mean survival as $\mathrm{CFU} / \mathrm{ml} \pm \mathrm{SEM}$. Pale bars $\square$ represent $0.1 \mu \mathrm{M}$ (low dose) MSD treatment/vehicle control, while black bars $\square$ represent $1 \mu \mathrm{M}$ (high dose) MSD treatment/vehicle control. Lat A: latrunculin A. Graphs are representative of 1 experiment, each with duplicate wells and 2 CFU plates per well. ${ }^{*} \mathrm{p}<0.05$ Lat A vs high dose ethanol vehicle control, one-way ANOVA with Newman-Keuls post test, $* * * \mathrm{p}<0.0010 .1 \mu \mathrm{M}$ paclitaxel vs low dose ethanol vehicle control, one-way ANOVA with Newman-Keuls post test. 
Figure 6.2.6
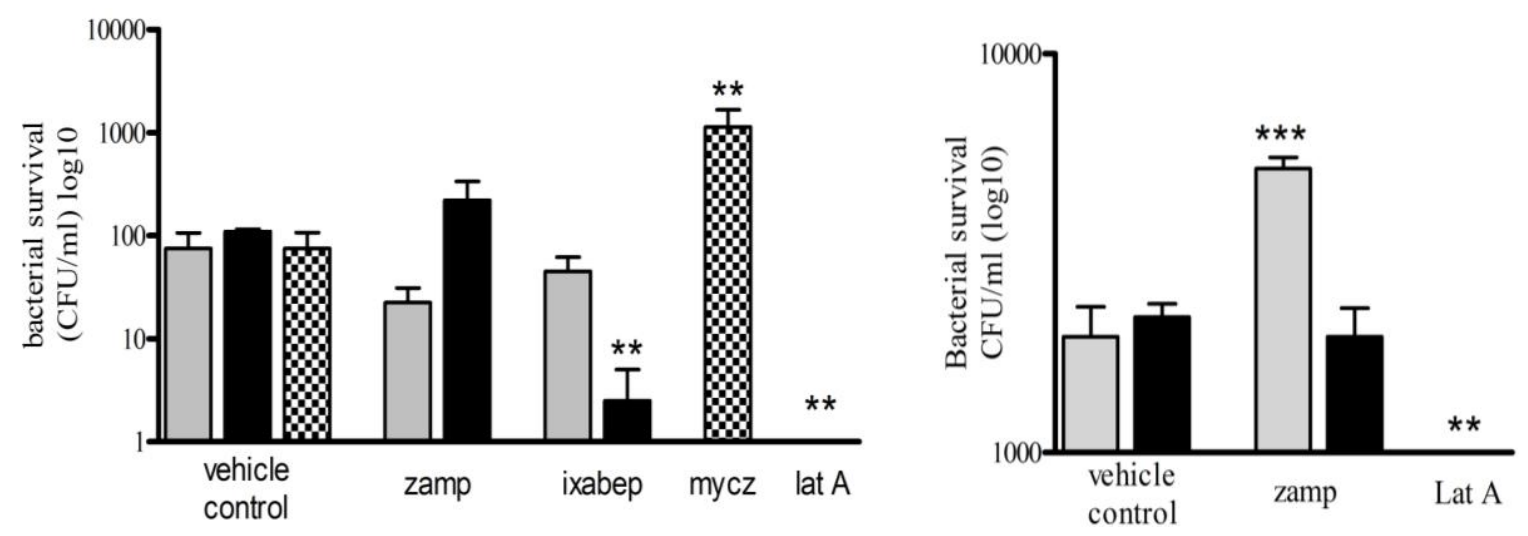

Figure 6.2.6 Bacterial survival in RAW264.7 macrophages treated with low and high dose MSD. (A) Bacterial survival was in the low CFU/ml range. Compared to vehicle controls, high dose ixabepilone significantly increases bacterial killing, while high dose mycothiazole significantly impairs bacterial killing. Latrunculin A (assay positive control) inhibits phagocytosis and bacterial survival is significantly impaired. (B) Bacterial survival was in the high CFU/ml range. Compared to vehicle control, low dose zampanolide treated macrophages significantly impair bacterial killing, while high dose zampanolide treatment does not alter killing ability. Latrunculin A significantly impairs bacterial survival as phagocytosis is impaired. In both experiments, all samples are gentamicin treated. For both graphs, bars represent mean survival as $\mathrm{CFU} / \mathrm{ml} \pm \mathrm{SEM}$. Pale bars $\square$ represent $0.1 \mu \mathrm{M}$ (low dose) MSD/vehicle treatment, black bars represent $1 \mu \mathrm{M}$ (high dose) MSD/vehicle treatment, while crossed bars $\$$ represent high dose mycothiazole and $0.1 \%$ DMSO vehicle control. Lat A: latrunculin A, Zamp: zampanolide, ixabep: ixabepilone, mycz: mycathiazole. Graphs are representative of 1 experiment, each with duplicate wells and 2 CFU plates per well. (A) **p<0.01 Lat A vs high dose vehicle control, **p<0.01 high dose ixabepilone vs high dose vehicle control, one-way ANOVA with Newman-Keuls post test. (B) $* * * \mathrm{p}<0.001$ low dose zampanolide vs low dose vehicle control, **p<0.01 Lat A vs high dose vehicle control, one-way ANOVA with Newman-Keuls post test 


\subsubsection{Effects of IFN- $\gamma$ pre-treatment on MSD treated RAW264.7 macrophages during bacterial killing}

As discussed in section 5.3, IFN- $\gamma$ plays an important role in activating macrophages during bacterial infections. In the presence of IFN- $\gamma$ macrophages are optimally activated. However we were interested in bactericidal ability of MSD treated macrophages and it seemed practical to study these effects when activation of macrophages was not optimal and the macrophage response to bacteria was solely due to the LPS stimuli. It was thought that removal of the optimal conditions might expose effects of MSD on macrophages, and that this lack of optimal conditions would also mimic in vivo situations where immune systems may be compromised and treatment with MSD was required.

As a result, we tested the effects of MSD on macrophages killing efficiency in the absence of IFN- $\gamma$ as compared to in the presence of IFN- $\gamma$ (Fig 6.2.7). It was possible that there would be inhibition to killing during MSD treatment due to lack of added stimuli from IFN- $\gamma$. However it was equally possible that with MSD such as paclitaxel or docetaxel, in the absence of IFN- $\gamma$, these drugs would compensate for the lack of stimuli and maintain bactericidal activity due to their immunomodulatory properties (Chan et al., 2000; Si et al., 2003). In addition, based on previous studies where treatment with IFN- $\gamma$ has led to resistance towards colchicine effects (Khandani et al., 2007), we tested the effects of colchicine treatment on bacterial killing in the presence and absence of IFN- $\gamma$. 
Figure 6.2.7
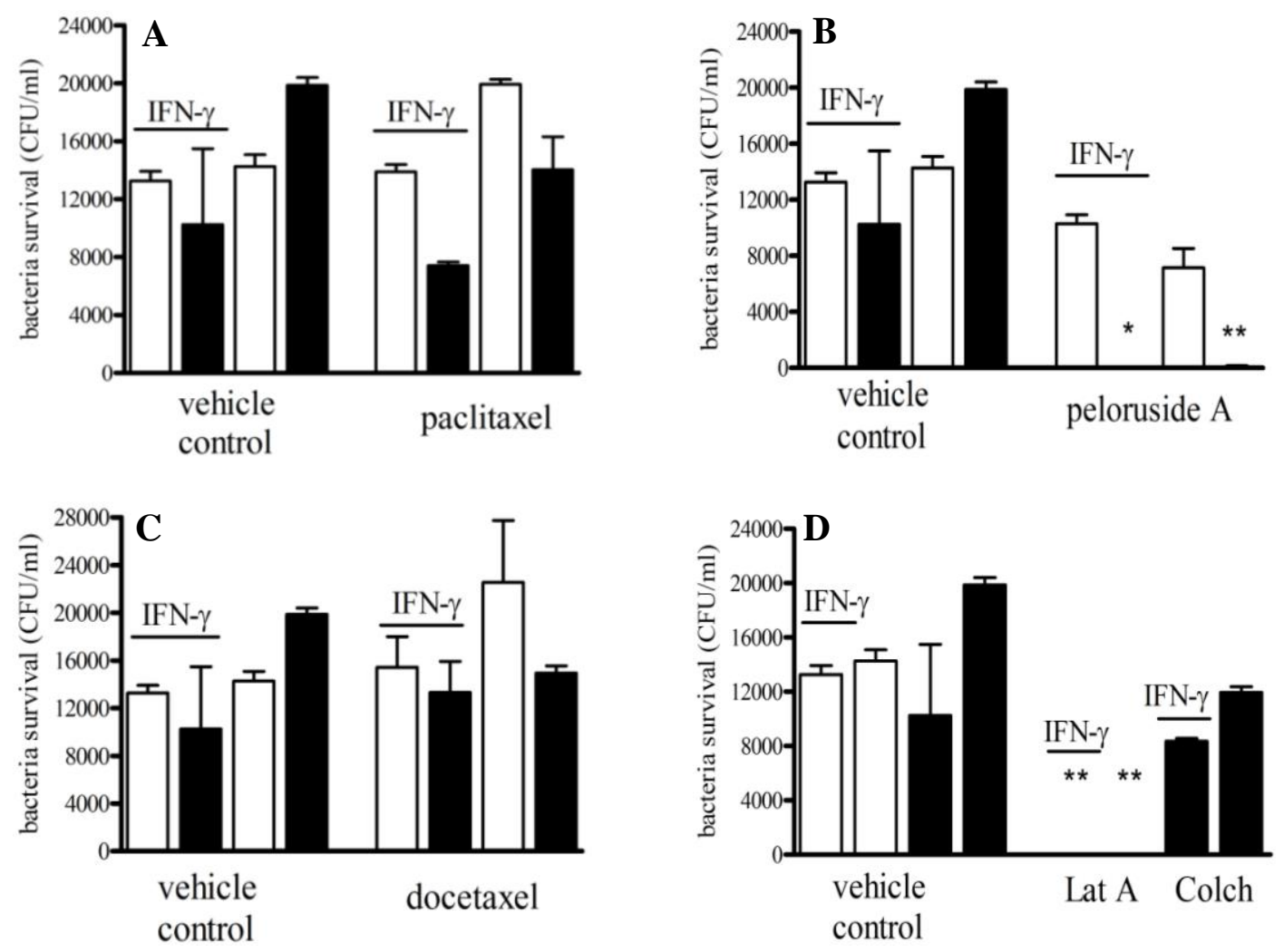

Figure 6.2.7 Effects of IFN- $\gamma$ pre-treatment on bacterial killing by MSD treated RAW264.7 macrophages. Macrophages were either pre-treated overnight with $100 \mathrm{U} / \mathrm{ml}$ of IFN- $\gamma$ or the same volume of media alone. The absence or presence of IFN- $\gamma$ did not cause a significant alteration to macrophage killing ability in the presence of (a) paclitaxel, (c) docetaxel, or (d) colchicine. In contrast, at high doses of peloruside A and Latrunculin A there was a significant increase in bacterial killing as compared to vehicle control irrespective of IFN- $\gamma$ presence. Bars represent mean survival as CFU/ml \pm SEM. White bars $\square$ represent $0.1 \mu \mathrm{M}$ (low dose) MSD/vehicle treatment, while black bars $\square$ represent $1 \mu \mathrm{M}$ (high dose) MSD/vehicle treatment. Lat A: latrunculin A, colch: colchicine. Graphs are representative of 1 experiment, each with duplicate wells and 1 CFU plate per well. ${ }^{*} \mathrm{p}<0.051 \mu \mathrm{M}$ peloruside $\mathrm{A}, \mathrm{IFN}-\gamma$ vs high dose ethanol vehicle control, ${ }^{*} \mathrm{*}<0.011$ $\mu \mathrm{M}$ peloruside $\mathrm{A}$, no IFN- $\gamma$ vs high dose ethanol vehicle control, **p<0.01 $10 \mu \mathrm{M}$ latrunculin A vs high dose vehicle control with and without IFN- $\gamma$, one-way ANOVA with Newman-Keuls post test. 


\subsection{Discussion}

Bacterial killing is an important aspect of phagocytosis. Binding, ingestion and killing of pathogens are all aspects of macrophage bactericidal activity, however while an MSD treated macrophage may be able to ingest bacteria, it might be impaired while killing it. Additionally, while it has been well established that microtubules are important in phagocytosis (Kaplan, 1977; Toyohara et al., 1989; Blocker et al., 1997; Blocker et al., 1998; Khandani et al., 2007), there are few studies that address the effects of MSD induced microtubule stabilization on macrophage bactericidal abilities. Based on this, the main aim of these experiments was to distinguish killing ability from phagogcytic ability and understand the effects of MSD treatment on the macrophage ability of bacterial killing. We were interested in assessing if treatment with MSD would increase or decrease bactericidal ability in macrophages, especially since phagocytosis was not impaired.

Initially we tested two concentrations of bacteria for suitable infection rates in RAW264.7 macrophages. Bacterial phagocytosis occurred at MOI values of 10:1 and 25:1 however, an MOI of 25 resulted in slightly higher amounts of internalized, live bacteria. While this value was not significantly greater than the levels of internalization at MOI 10, we decided to use the higher MOI for infections. This was to ensure that upon treatment with MSD there was sufficient bacterial challenge to the immune system. If bacterial levels were low, it could be possible that in the presence of MSD there would be no effect on killing ability; therefore we chose to use a higher MOI.

It is known that macrophage phagocytosis of bacterial and other pathogens occurs via complement or FcR mediated pathways (Aderem et al., 1999; Underhill et al., 2002). We investigated the requirement of opsonisation for phagocytosis, by opsonising bacteria with 
BALB/c mouse serum which contained opsonins and IgG antibodies. However we found that E.coli phagocytosis was not affected by presence or absence of serum opsonins. This was also seen in another study (Sukumaran et al., 2003) and suggests that E.coli phagocytosis does not need complement or antibody and that TLR-LPS stimulation is sufficient to trigger phagocytosis. IFN- $\gamma$ pre-treatment to activate RAW264.7 macrophages for optimal phagocytosis was also investigated, but the rates of phagocytosis and killing were not significantly affected irrespective of IFN- $\gamma$ pre-treatment.

We found that the gentamicin antibiotic treatment in the infection assay was successful in killing extracellular bacteria as expected. This was confirmed by the lack of colony growth when bacteria alone were treated with gentamicin, as well as the reduction in $\mathrm{CFU}$ numbers when comparing macrophages and bacteria alone with macrophages and bacteria with gentamicin treatment. The gentamicin survival assay enabled us to study macrophage killing ability, because bacteria that are phagocytosed are protected from antibiotic killing. However, bacterial killing occurs inside the macrophage as well, therefore measuring bacteria after a short period of time (between 90 to 120 minutes) lessens the chance of bacterial replication or excessive bacterial killing within the macrophage (Campbell et al., 1994; Laroux et al., 2005). When comparing CFU counts from macrophages that are non-drug treated to MSD treated macrophages, there are two possible results; i)CFU counts are similar in MSD treated and non treated macrophages indicating that there was no increase in killing ability or ii)CFU counts are lower in MSD treated macrophages indicating that there was an increase in killing ability. Thus this assay gives us an idea of the enhancement or impairment to killing capacity. Relating this back to the presence of MSD allows understanding the effects of MSD on bacterial killing. 
The results of MSD treatment on bacterial killing were remarkable and suggested different levels of inhibition in bacterial killing. The inhibitory effects of MSD on bacterial killing were also only seen when bacterial CFU were low. This was interesting because the MOI of all assays were 25:1 and macrophages were seeded at the same concentrations and in the same experimental media. Results from the MSD killing assays were presented as relating to high and low CFU to avoid masking the effects of MSD seen in the presence of low CFU values. The difference in CFU numbers is hard to explain as assay results seemed to fall into these two categories without a corresponding change in assay conditions. It may be possible that at some stage of cell line passage macrophages were more competent and able to kill bacteria efficiently resulting in low CFU values irrespective of MSD presence. It could also be possible that MSDs stimulated killing to a greater extent and caused low CFU numbers, however the low CFU was also seen in non MSD treated macrophages. Therefore, it seems more likely that the variation in CFU results was due to an unknown interaction between macrophages and bacteria. As cells were used only between 2 and 10 passages, it is unlikely that some experiments had mature, activated cells, which were competent in bacterial killing and other experiments had younger cells at low passage that were not as efficient at bacterial killing. We do not have any explanations as to why killing assays sometimes had low and other times had high CFU numbers. However, it still stands that we saw greater effects of MSD treatment when CFU counts were low.

When treated with paclitaxel, peloruside A or docetaxel at low or high doses, there was no significant change in bacterial killing when bacterial survival was in the high CFU category. There was a significant change, with increased killing in latrunculin treated macrophages, but this was due to latrunculin blocking phagocytosis. This resulted in all the 
bacteria being extracellular and gentamicin treatment resulted in bacterial killing and lack of CFU.

In contrast, with high CFU counts and treatment with low dose zampanolide, there was a significant inhibition of killing, while high dose zampanolide restored the killing ability of macrophage to levels similar to those of vehicle treated macrophages. This suggests that zampanolide may have some stimulatory effect on macrophages similar to paclitaxel. However from pHrodo phagocytosis assays, it can be seen that there is a significant dose dependent inhibition of phagocytosis in the presence of zampanolide. Taken together these results suggest that zampanolide treatment not only impairs phagocytosis, but also impairs killing. At high CFU levels, and low doses of zampanolide, there is limited phagocytosis, and bacteria that are taken up cannot be killed. Additionally, with high dose zampanolide and high CFU, bacterial uptake is significantly inhibited and bacteria that might be taken up are not killed efficiently. A possible explanation for this could be the binding site of zampanolide, which is unknown (Miller et al., 2010) interfering with LPS binding of microtubules, leading to loss of sufficient stimulation and impaired phagocytosis. As mentioned previously, zampanolide could also affect actin, and this combined with microtubule effects would additionally impair endocytic trafficking and affecting killing (Apodaca, 2001).

When CFU counts were low and cells were treated with paclitaxel, peloruside A or docetaxel, there was significant alteration to killing in the presence of low dose paclitaxel treated macrophages. The CFU counts were significantly higher; indicating that these paclitaxel treated cells had impaired bactericidal activity. The data from pHrodo phagocytosis demonstrates that there is no increase in phagocytosis, which indicates that 
the high CFU counts are not due to increased phagocytosis, but rather due to decreased killing ability. Taken together, the low and high CFU data suggest that if cells phagocytose high numbers of bacteria, irrespective of MSD treatment, killing is impaired. This is because the high CFU counts are seen with vehicle treated macrophages as well as MSD treated macrophages. However if for some unknown reason, cells phagocytose less bacteria, then paclitaxel treatment affects how well these bacteria are killed. With low dose paclitaxel, there was an inhibition to killing, and this was alleviated in the presence of high dose paclitaxel, possibly due to LPS mimicry exhibited by paclitaxel. These results are extremely interesting, especially when considering the LPS mimicry of paclitaxel. Similarly to LPS, paclitaxel can cause activation of TLR-4 by binding to TLR-4 in combination with the accessory protein MD-2 (Kawasaki et al., 2000; Kawasaki et al., 2001a). This interaction is MD-2 dependent and species specific and thus paclitaxel displays LPS mimicry only in murine macrophages and monocytes (Shimazu et al., 1999; Kawasaki et al., 2001a; Zimmer et al., 2008). It would be prudent to study bactericidal effects in paclitaxel treated human macrophage cell line such as 2MAC (Dialynas et al., 1997) as there would be a lack of LPS mimicry in these cells. It is likely that due to paclitaxel's lack of LPS mimicry in human cells, there will be inhibition to bacterial killing. Furthermore, these studies are in vitro and a more detailed understanding would be gained from doing these studies on human macrophage cell lines. Testing these MSD in vivo in mice, would also provide a more in depth picture of the effects of the MSD on macrophage phagocytosis and killing.

When studying the effects of the novel compounds ixabepilone, and mycothiazole, we found that in the presence of high doses of ixabepilone, bacterial killing was enhanced, 
but at low doses the killing efficiency was similar to vehicle treated macrophages. Ixabepilone competes for the same binding site as paclitaxel, however it is active in paclitaxel resistant cells (Bollag et al., 1995; Goodin et al., 2004), suggesting that in RAW264.7 cells which are comparatively resistant to paclitaxel, the effects of ixabepilone would be more pronounced. This is seen when high dose ixabepilone significantly increases macrophage killing ability. It is unknown if ixabepilone exhibits LPS mimicry and it would be worthwhile to determine this as paclitaxel's semi-synthetic analogue, docetaxel does not exhibit LPS mimicry. We carried out this assay once, and the CFU obtained were in the low category, it is possible that if $\mathrm{CFU}$ were to be higher, there would be no effect of ixabepilone treatment. The effects of mycothiazole are also interesting. There was a significant inhibition to bacterial killing in the presence of high dose mycothiazole. While the intracellular targets for mycothiazole are unknown (Miller et al., 2010), these results combined with pHrodo results suggest that mycothiazole may possibly affect microtubule stability similar to MSDs. A recent study has found that mycothiazole treatment led to reduced ROS production (unpublished data; Meyer, K. et al. 2010). If initial inflammatory mediators are suppressed, this may explain the lack of efficient killing in macrophages. In the same study, it was also proposed that mycothiazole may have multiple targets (unpublished data; Meyer, K. et al. 2010), however the researchers found that mycothiazole did not affect cell cycle progression, suggesting that microtubules were not targeted by this compound. In this case, there could be an alternate mechanism by which mycothiazole affects bacterial killing and given the variety of responses and activity of mycothiazole in differing cell lines (unpublished data; Meyer, K. et al. 2010). this is not unlikely. Additionally, due to limited supply of both ixabepilone and mycothiazole, they were 
assayed once in our studies and it would be beneficial to replicate these assays to confirm our results.

Similarly to the pHrodo assays, we tested the effects of IFN- $\gamma$ treatment on efficiency of bacterial killing in the presence of MSD. There was no significant alteration to bacterial killing ability in the presence or absence of IFN- $\gamma$ in macrophages treated with paclitaxel or docetaxel. Levels of survival were comparable in MSD and vehicle treated cells, irrespective of IFN- $\gamma$ treatment. Overall there was a trend of increased bacterial survival, indicating decreased killing ability, in the absence of IFN- $\gamma$, irrespective of drug treatments. This is expected as IFN- $\gamma$ activates macrophages to respond to bacterial stimulus (Young et al., 1995; Schroder et al., 2004). In the presence of peloruside A, latrunculin A and colchicine however, there was significant alterations to bacterial killing. When macrophages were treated with low doses of peloruside A, irrespective of IFN- $\gamma$ treatment, there was no significant inhibition to bacterial killing ability. However, in the presence of high dose peloruside $\mathrm{A}$, there was a significant increase in bacterial killing. The presence of IFN- $\gamma$ significantly increased killing ability at high doses of peloruside A, however in the absence of INF- $\gamma$, the increase in killing ability was much more significant. This suggests that in the absence of IFN- $\gamma$, peloruside A stimulates cells to maintain killing ability. The increased significance in the peloruside A, non IFN- $\gamma$ treated cells, could be due to the non IFN- $\gamma$, vehicle control treated cells exhibiting high bacterial survival. However this still supports the theory that in the absence of IFN- $\gamma$, bacterial killing is sub-optimal and treatment with peloruside A leads to rescue of the killing efficiency. In latrunculin A treated macrophages, as expected, there was a significant decrease in bacterial survival, irrespective of IFN- $\gamma$ treatment. In colchicine treated macrophages, while there was no 
significant alteration to killing ability, in the presence of IFN- $\gamma$ bacterial survival was lower than in the absence of IFN- $\gamma$. This is similar to results seen with pHrodo assays, where colchicine treatment had significantly decreased uptake of bacteria irrespective of IFN- $\gamma$ treatment. We did not find colchicine treatment to inhibit bacterial killing and survival levels were comparable to the level of survival seen with the MSD treated samples. Colchicine is a microtubule destabilizer, and we would expect alterations to bacterial killing as transport pathways would be affected. However the lack of inhibition seen in bacterial killing could be due to the presence of a stable pool of cytoplasmic microtubules that are able to maintain cellular function (Schroer et al., 1991).

Overall, we found that bacterial killing was not significantly affected in the clinically used MSD paclitaxel and docetaxel. However, we found peloruside A could significantly increase bacterial killing if optimal stimulation was absent. We also found that zampanolide treatment affects the ability of macrophages to phagocytose and kill bacteria efficiently and this suggests that if used clinically there is likely to be altered immune responses in the presence of bacteria. Similarly, we found that ixabepilone treatment enhances microbial killing, while mycothiazole impaired microbial killing. With all of these assays, the results are preliminary due to limited compound availability and repeating these assays is required. 
Chapter 7: Effects of MSD treatment on cytokine production in RAW264.7 macrophages

\section{CHAPTER 7: EFFECTS OF MSD TREATMENT ON CYTOKINE PRODUCTION DURING BACTERIAL KILLING IN RAW264.7 MACROPHAGES.}

Macrophage stimulation with LPS results in production of the inflammatory cytokines TNF- $\alpha$, IL-12 and NO (Tracey et al., 1994; Ma et al., 1996; Sosroseno et al., 2002). These cytokines play an important role in bactericidal activities and aid in clearance of bacteria. MSD can alter cytokine profiles and treatment with these drugs can also result in immunostimulatory effects (Mullins et al., 1999; Tsavaris et al., 2002; Crume et al., 2007). The stabilization of microtubules due to MSD treatment can also lead to inhibition in vesicle trafficking and thus affect release of cytokines (Murray et al., 2005). We were interested in studying the effects of MSD treatment on macrophage ability of phagocytosis and bacterial killing. The ability of macrophages to respond to pathogens and resolve infections is linked to cytokine signalling and optimal macrophage responses involve cytokines (Zhang et al., 2008).

For this reason, we decided to investigate the effects of MSD treatment on cytokine production during phagocytosis and bacterial killing. We were interested in changes to the cytokine profile that would help explain any enhancement or inhibitions in macrophage phagocytic and bactericidal ability during MSD treatment. We looked at changes to TNF- $\alpha$ production as this cytokine is produced earliest in response to bacterial and LPS stimulation (Tracey et al., 1993). We also looked at nitric oxide production which is produced in response to bacterial LPS (Stuehr et al., 1985). The maximum incubation time for most of our bacterial phagocytic assays were 3 hours or less. TNF- $\alpha$ was the only cytokine with optimal production levels that was measurable within this time frame. There was limited nitric oxide production at this early time point and IL-12 production was negligible. 
Chapter 7: Effects of MSD treatment on cytokine

production in RAW264.7 macrophages

\subsection{Interactions between TNF- $\alpha$ and paclitaxel in LPS stimulated RAW264.7} macrophages.

\subsubsection{Introduction}

Paclitaxel stimulates an inflammatory profile in macrophages, leading to a release of TNF- $\alpha$, IL-12 and NO (Fitzpatrick et al., 2003). Previous studies in our lab using BMDM, have demonstrated that in the presence of limiting doses of LPS, paclitaxel can lower the amount of TNF- $\alpha$ produced, leading to an anti-inflammatory profile (Crume et al., 2007). Paclitaxel has also been seen to increase metabolism levels in LPS-stimulated BMDM (Crume et al., 2007). Additionally, as mentioned before, paclitaxel can mimic the effects of LPS in murine macrophages (Ding et al., 1990b). We therefore decided to investigate the ability of LPS to stimulate TNF- $\alpha$ production in RAW264.7 and J774.2 murine macrophages over a 2 hour and 8 hour incubation (Fig 7.1.1). We were interested in determining optimal times for TNF- $\alpha$ measurement as well as concentrations of LPS that could be considered as low or high doses depending on their ability to induce TNF- $\alpha$ production. We also looked at the rate of metabolism of these cells in the presence of LPS (Fig 7.1.2). We then studied the effect of paclitaxel treatment on TNF- $\alpha$ production (Fig 7.1.3) to see if limiting LPS doses caused paclitaxel to exhibit an anti-inflammatory profile as previously seen. We also studied the corresponding 2 and 8 hour cell metabolic levels (Fig 7.1.4) when the cells were stimulated with LPS and paclitaxel. 


\subsubsection{Results}

\section{Figure 7.1.1}

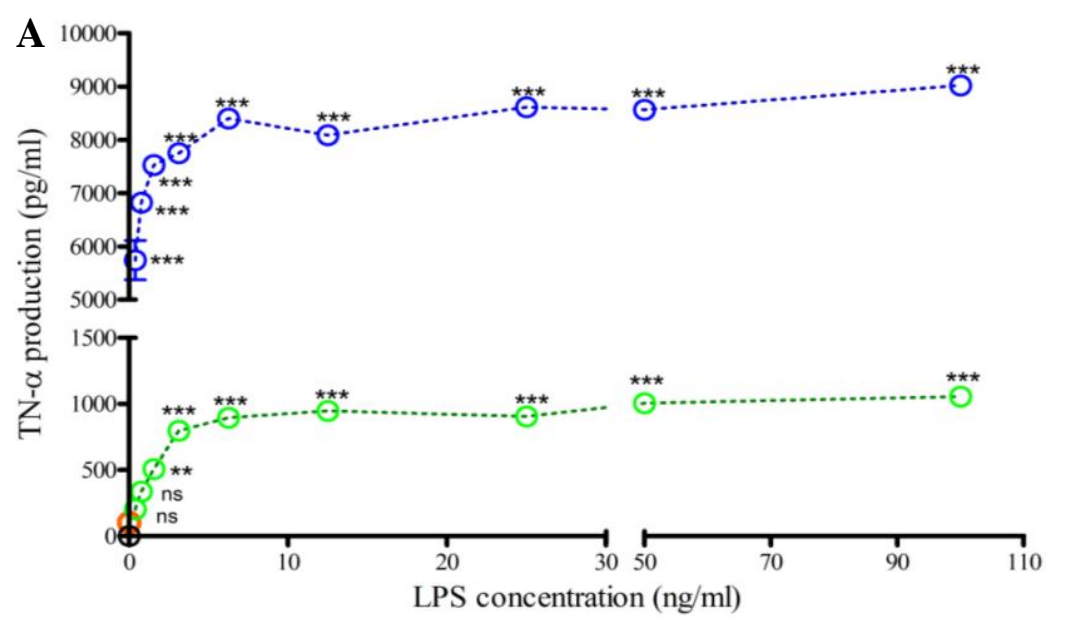

Figure 7.2.1A Figure Legend

J774.2 cells

- - - J774.2 cells + LPS

O RAW264.7 cells

- - - RAW264.7 cells + LPS

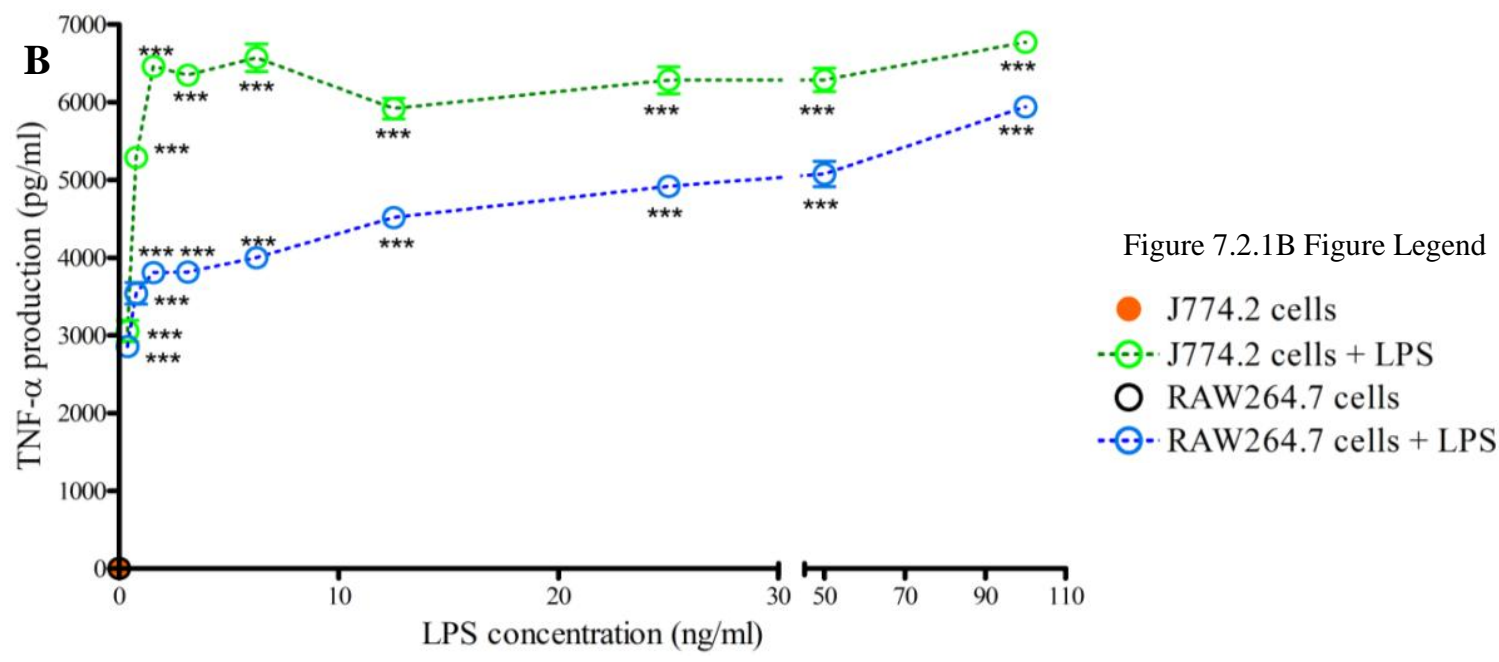

Figure 7.1.1 TNF- $\alpha$ production in (A) 2 hour LPS-stimulated RAW264.7 and J774.2 macrophages and (B) 8 hour LPS-stimulated RAW264.7 and J774.2 macrophages. Cells with a concentration of $2 \times 10 \% \mathrm{ml}$ were seeded at $50 \mu \mathrm{L} /$ well, with a range of concentrations of LPS, starting at $100 \mathrm{ng} / \mathrm{ml}$ serial diluted down. (A) At 2 hours, RAW264.7 and J774.2 cells produce significantly more TNF- $\alpha$ when compared to controls. RAW264.7 cells are significantly more responsive to LPS as compared to J774.2 cells. (B) At 8 hours, RAW264.7 and J774.2 cells produce significantly higher levels of TNF- $\alpha$ in comparison to controls. Levels of TNF- $\alpha$ production in J774.2 cells at 8 hours are similar to the levels of TNF- $\alpha$ seen in RAW264.7 cells at 2 hours. For both graphs, data points are representative of mean \pm SEM of triplicate wells from two experiments. $* * p<0.01$, $* * * \mathrm{p}<0.001$, RAW264.7 or J774.2 + LPS vs cells alone, two-way ANOVA with Bonferroni post test. 
Figure 7.1.2

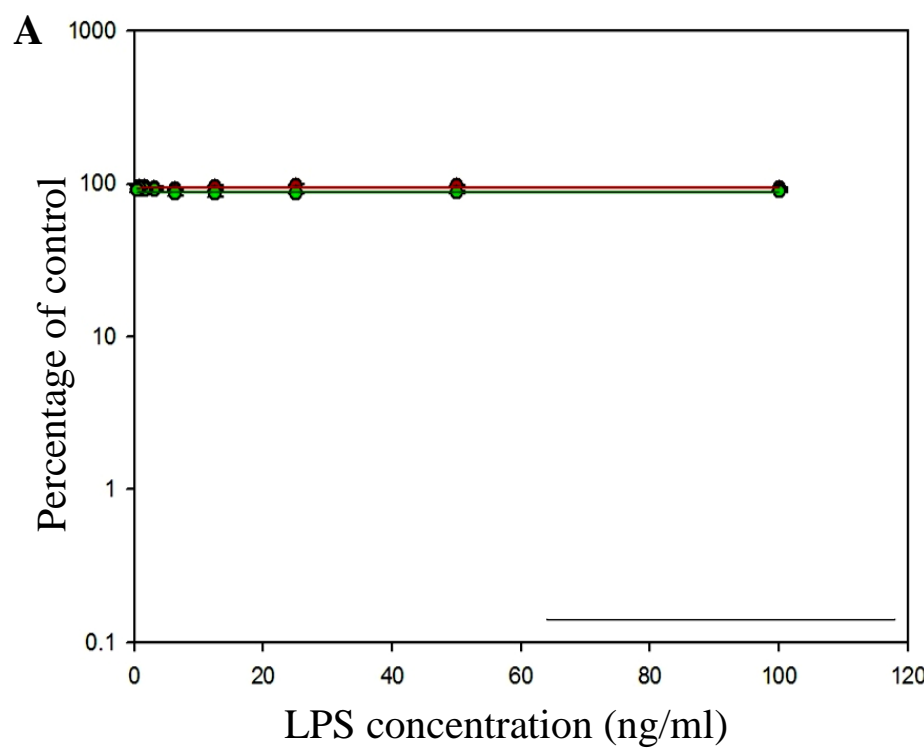

Figure 7.2.2A: Figure Legend

2 hours

- RAW264.7 + LPS

- J774.2 + LPS

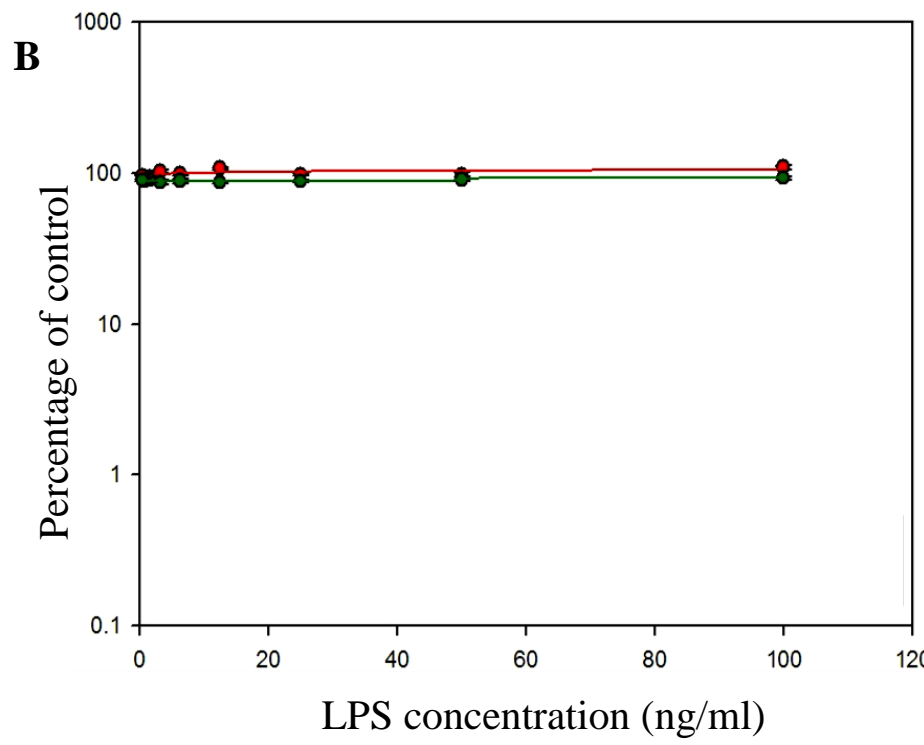

Figure 7.2.2B: Figure Legend

8 hours

- RAW264.7 + LPS

- J774.2 + LPS

Figure 7.1.2 RAW264.7 and J774.2 cells exhibit a constant rate of metabolism in the presence of (A) 2 hours or (B) 8 hours LPS-stimulation. RAW264.7 and J774.2 cells do not significantly alter their metabolic rates in response to a range of LPS concentrations over (A) 2 hours or (B) 8 hours. Data points represent rate of metabolism as a percentage of control from untreated cells. Each point is mean \pm SEM of three replicates each from two experiments. $\mathrm{p}>0.05$, two-way ANOVA with Bonferroni post test. 
Figure 7.1.3

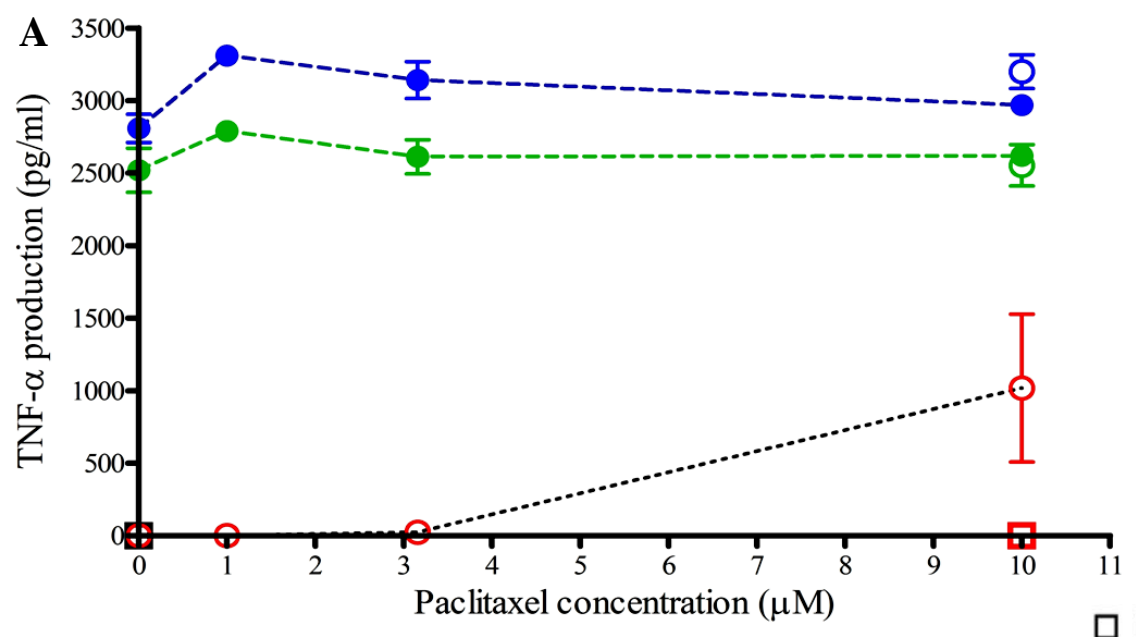

Figure 7.2.3A, B:

Figure Legend

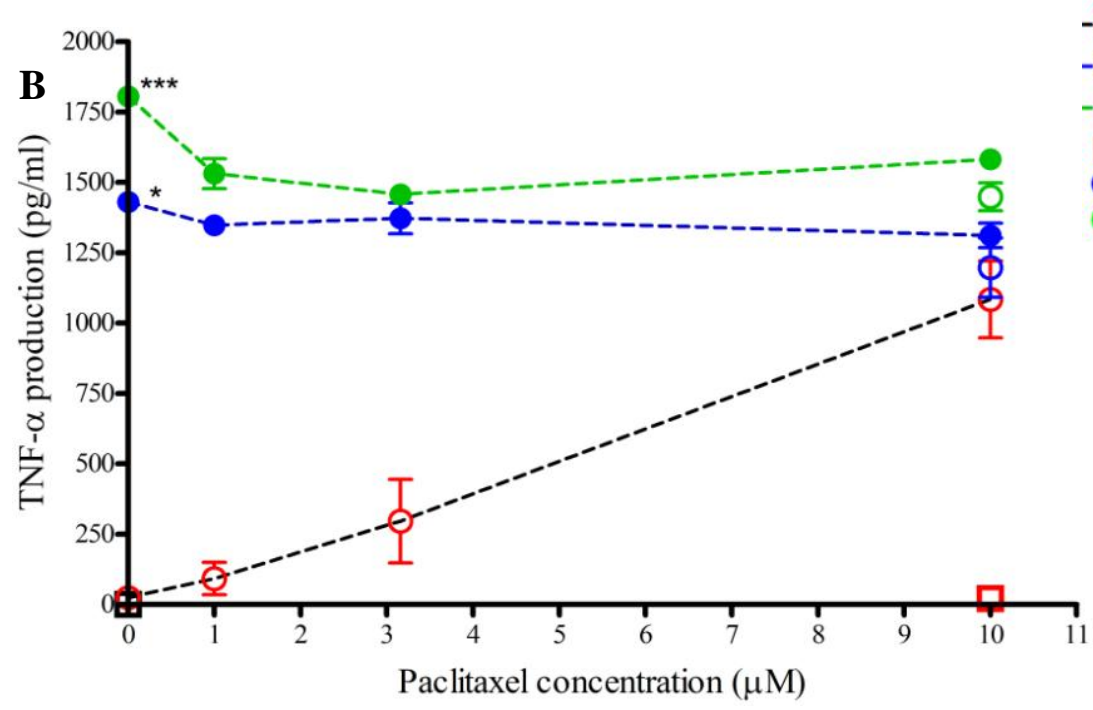

$-\Theta$ - Cells

- LPS $0.3 \mathrm{ng} / \mathrm{ml}$

- LPS $5 \mathrm{ng} / \mathrm{ml}$

$\square$ cells + vehicle

O cells + LPS $0.3 \mathrm{ng} / \mathrm{ml}+$ vehicle

cells + LPS $5 \mathrm{ng} / \mathrm{ml}+$ vehicle

Figure 7.1.3 Effect of paclitaxel on TNF- $\alpha$ production from (A) 2 hours and (B) 8 hours LPS stimulated RAW264.7 macrophages. Cells were cultured with $0.3 \mathrm{ng} / \mathrm{ml} \mathrm{LPS}$ (low dose) or $5 \mathrm{ng} / \mathrm{ml}$ LPS (high dose) and a range of paclitaxel concentrations. (A) There were no significant changes to TNF- $\alpha$ production at 2 hours as compared to LPS stimulated vehicle control. (B) 8 hours paclitaxel treatment did not alter TNF- $\alpha$ production, however at both doses of LPS alone, there was a significant increase in TNF- $\alpha$ production as compared to LPS stimulated vehicle control. For both graphs, data points are representative of mean \pm SEM of triplicate wells from two experiments. ${ }^{*} \mathrm{p}<0.05, * * * \mathrm{p}<0.001$, cells with LPS vs cells with LPS and vehicle, two-way ANOVA with Bonferroni post test. 
Figure 7.1.4
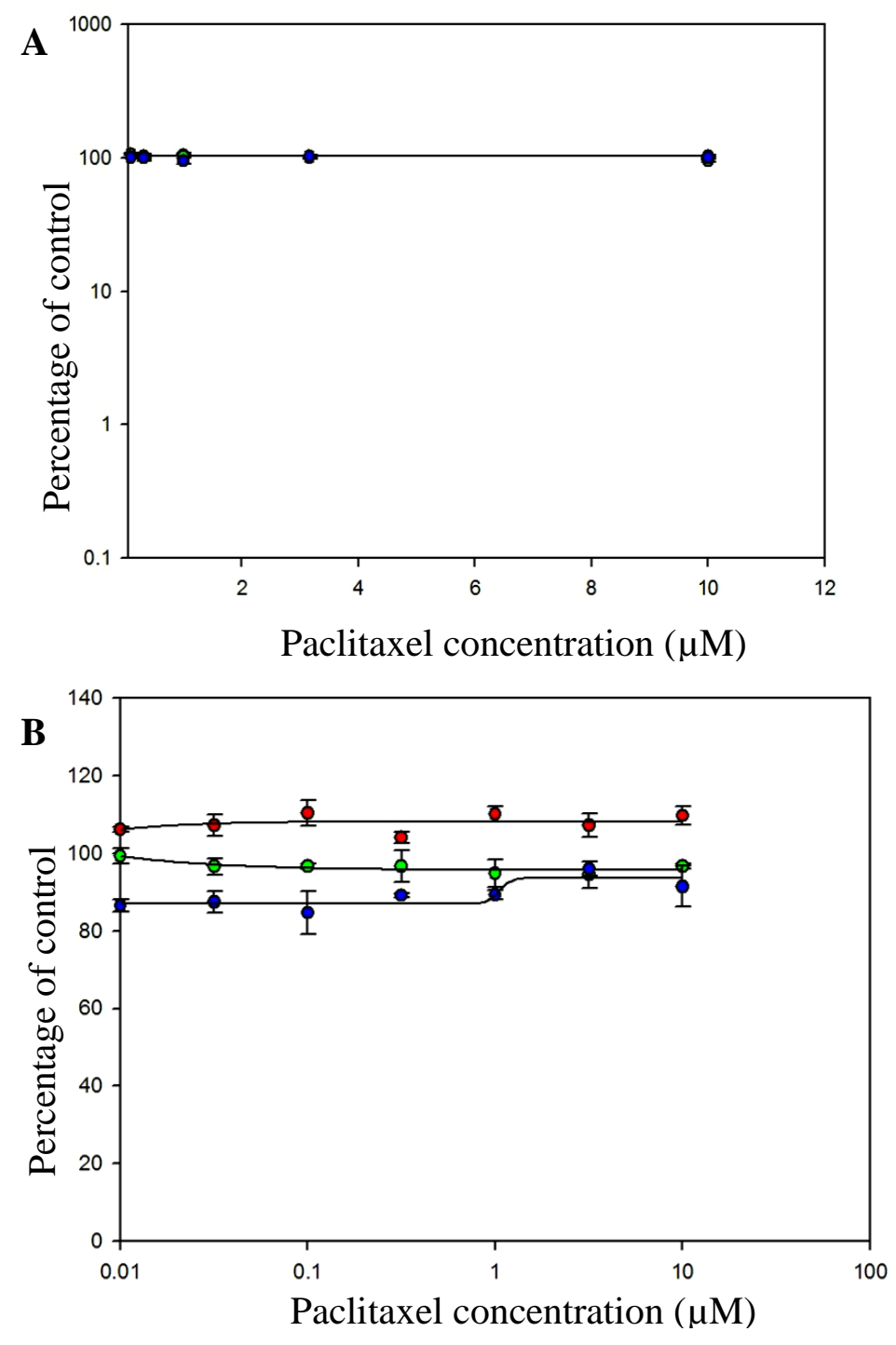

Figure 7.2.4 A, B: Figure Legend

- cells with paclitaxel

- $0.3 \mathrm{ng} / \mathrm{ml}$ low dose LPS

- $5 \mathrm{ng} / \mathrm{ml}$ high dose LPS

Figure 7.1.4 Rate of metabolism in paclitaxel treated, LPS stimulated RAW264.7 cells at (A) 2 hours and (B) 8 hours. (A) RAW264.7 macrophages exhibit extremely comparable rates of metabolism at 2 hours, irrespective of treatment with paclitaxel alone or paclitaxel and low or high dose LPS (B) There is no significant change in the metabolic rate of RAW264.7 cells at 8hours when stimulated with paclitaxel alone or paclitaxel and low or high dose LPS. However, at 8 hours there is a variation in metabolic rates at each treatment, which is not seen at the 2 hours treatment. RAW264.7 macrophages treated with $5 \mathrm{ng} / \mathrm{ml}$ LPS have the highest rate of metabolism at 8 hours, followed by $0.3 \mathrm{ng} / \mathrm{ml}$ LPS treatment and paclitaxel treatment. For both graphs, rate of metabolism is measured as a percentage of control from untreated cells. Each point is mean \pm SEM of three replicates each from two experiments. $\mathrm{p}>0.05$, two-way ANOVA with Bonferroni post test. 
Chapter 7: Effects of MSD treatment on cytokine production in RAW264.7 macrophages

\subsubsection{Discussion}

This set of assays was done to study the effects of paclitaxel on TNF- $\alpha$ production in the presence of LPS stimulation. Initially we tested the responsiveness of both the macrophage cell lines RAW264.7 and J774.2 to LPS stimulation. We found that RAW264.7 macrophages are much more sensitive to LPS stimulation, seen by the high levels of TNF- $\alpha$ produced in RAW264.7 cells. At 2 hours RAW264.7 cells produce approximately 9 times more TNF- $\alpha$ as compared to J774.2 cells. However by 8 hours, the J774.2 cells produce similar levels of TNF- $\alpha$ as seen with the RAW264.7 cells in 2 hours. Both the RAW264.7 and J774.2 cells showed increasing levels of TNF- $\alpha$ production before levelling out, indicating that increasing LPS only caused increases in TNF- $\alpha$ up to certain concentrations, after which increasing LPS did not correspond to increasing TNF- $\alpha$ levels. While the J774.2 cells took longer to produce TNF- $\alpha$, reaching maximum production levels at 8 hours, the RAW264.7 cells exhibited decreased TNF- $\alpha$ level at 8 hours. This could be due to reabsorption of the high amounts of TNF- $\alpha$ produced or it could be a combination of reabsorption and declining TNF- $\alpha$ production due to cellular energy being used up for the initial burst of TNF- $\alpha$ production. There was however, no corresponding increase in metabolic rate of RAW264.7 cells at 2 or 8 hours.

From the graphs, the $\mathrm{ED}_{50}$ values (LPS concentration at which half-maximal TNF- $\alpha$ is produced) for RAW264.7 cells and J774.2 cells at 2 hours were approximately $1 \mathrm{ng} / \mathrm{ml}$ and $2 \mathrm{ng} / \mathrm{ml}$ LPS respectively, while at 8 hours, the $\mathrm{ED}_{50}$ values were $1.5 \mathrm{ng} / \mathrm{ml}$ in RAW264.7 and $0.5 \mathrm{ng} / \mathrm{ml}$ in J774.2 cells. This is comparable to previous studies from our lab, where the approximate $\mathrm{ED}_{50}$ value of $1 \mathrm{ng} / \mathrm{ml} \mathrm{LPS}$, was seen in primary BMDM when measuring TNF- $\alpha$ production (Robinson, 2009). This is interesting as it indicates that the 
same concentration of LPS is required for peak TNF- $\alpha$ production in all the three cell types, irrespective of LPS stimulation times, which was 2 and 8 hours in our assays and 8 hours in the work by Robinson, 2009.

Based on the initial assays, we chose RAW264.7 cells for the remainder of the studies as they were more sensitive to LPS stimulation. However titrating LPS doses low enough to get a good distinction between high and low doses was challenging. The low dose chosen was $0.3 \mathrm{ng} / \mathrm{ml}$ which still led to comparably high TNF- $\alpha$ production. Additionally there was not a huge distinction between levels of TNF- $\alpha$ produced with $0.3 \mathrm{ng} / \mathrm{ml}$ or $5 \mathrm{ng} / \mathrm{ml} \mathrm{LPS}$. While retrospectively it seems as though it may have been better to pick the J774.2 cell line as these cells were not as LPS sensitive, the LPS dosage was equally hard to titrate in these cells with not much distinction between low and high doses of LPS in terms of TNF- $\alpha$ production. We found that the metabolic rate of RAW264.7 cells at 2 hours was unaffected by the presence of paclitaxel, with high or low dose LPS. Previous studies (Crume et al., 2007), have shown that paclitaxel causes a metabolic increase in BMMO however this increase was measured after a 72 hour incubation and it is likely that at the early time point measured there would be no increase in metabolic activity despite there being increased TNF- $\alpha$ production.

In contrast, at 8 hours, the metabolic rate of cells treated with high dose LPS was higher in comparison with metabolic rates for cells treated with low dose LPS and paclitaxel or paclitaxel alone. Cells treated with low dose LPS for 8 hours, had metabolic rates similar to that of cells treated with high or low dose LPS for 2 hours. Additionally, cells treated with paclitaxel showed a marginal decrease in metabolic rate at 8 hours as compared to treatment at 2 hours or compared to treatment with high dose LPS for 8 hours. 
However, none of these observed changes in metabolic rates were significant. We also confirmed in chapter 3 that an 8 hour treatment with paclitaxel alone caused a significant change in cell metabolism compared to 2 hours. This data together indicates that increasing LPS stimulus or paclitaxel exposure does not increase cell metabolism or affect cell viability over short incubation times. However at 8 hours, while LPS stimulus increases cell metabolism due to activation of inflammatory responses (Crume et al., 2007), treatment with paclitaxel alone seems to affect cell viability as determined by decreases in cell metabolism. One possible reason for this could be that the cells start to show susceptibility to paclitaxel, also they lack the extra LPS stimulation and this could possibly affect metabolism as well. There may be increases in metabolism if cells were treated with LPS and paclitaxel for longer, however as noted in chapter 3 , the time course of this incubation would be limited due to the cytotoxic effects of paclitaxel on proliferating cells.

Compared to vehicle controls, paclitaxel did not cause any inhibition to TNF- $\alpha$ production when cells were treated with low dose LPS and paclitaxel or high dose LPS and paclitaxel at 2 or 8 hours. An interesting observation made was that cells treated with low or high dose LPS alone for 8 hours produced significantly more TNF- $\alpha$ as compared to cells treated with the same dose of LPS and in the presence of ethanol as a vehicle control. The data suggests that TNF- $\alpha$ production is inhibited in the presence of ethanol, an effect that has been seen previously (Xie et al., 1995; Stoltz et al., 2000; Feng et al., 2002). The lack of inhibition to TNF- $\alpha$ production in the presence of limiting LPS and paclitaxel could be due to the high sensitivity of RAW264.7 cells to LPS stimulation. In previous studies paclitaxel treatment in the presence of LPS doses of $20 \mathrm{ng} / \mathrm{ml}$ or less caused a decrease in TNF- $\alpha$ production (Crume et al., 2007), however with LPS doses as low as $0.3 \mathrm{ng} / \mathrm{ml}$ we 
did not see a decrease in TNF- $\alpha$ production, once again indicating that RAW264.7 cells were extremely sensitive to LPS. As seen previously, we found that cells treated with paclitaxel alone were able to produce TNF- $\alpha$, indicating that paclitaxel displays LPS mimicry in murine macrophages.

Overall, the data from these assays suggested that compared to BMDM or J774.2 cells, RAW264.7 cells were extremely sensitive to LPS stimuli. We also found that paclitaxel or LPS stimulation alone or in combination did not increase cell metabolism in 2 hours. However an 8 hour treatment of paclitaxel alone seemed to decrease cell metabolism, which was an indicator of cell viability. The decrease in cell viability at 8 hours in the presence of paclitaxel seemed to be rescued by additional stimulation of high dose LPS for the same incubation period. We also found that in contrast to previous work in BMDM, paclitaxel treatment at limiting LPS doses did not decrease TNF- $\alpha$ production. 
Chapter 7: Effects of MSD treatment on cytokine production in RAW264.7 macrophages

\subsection{Changes in TNF- $\alpha$ and NO production during pHrodo phagocytosis in MSD} treated macrophages.

\subsubsection{Introduction}

This set of assays was carried out to study the effects of MSD treatment on cytokine production during bacterial phagocytosis. As mentioned earlier, macrophages produce TNF- $\alpha$ and NO in response to LPS stimuli or paclitaxel treatment (Stuehr et al., 1985; Ding et al., 1990b; Nakano et al., 1999; Bingle et al., 2002). Studies have also shown paclitaxel and docetaxel capable of modifying cytokine profiles (Tsavaris et al., 2002). Additionally, while peloruside A stabilizes microtubules similarly to paclitaxel; it does not share paclitaxel's ability to induce an inflammatory profile (Hood et al., 2001; Crume et al., 2007). There are few studies investigating the effects of peloruside A on cytokine production during bacterial phagocytosis. Furthermore, given the LPS mimicry displayed by paclitaxel, and the immunomodulatory effects of paclitaxel and docetaxel we were interested in investigating the interactions of MSD treatment and LPS stimuli on cytokine production during bacterial phagocytosis. Similarly to peloruside A, docetaxel does not exhibit LPS mimicry, and we were interested in the possible anti-inflammatory effects of these actives.

As described in the methods, supernatants were collected during infection assays with pHrodo, and stored at $-20^{\circ} \mathrm{C}$ for cytokine analysis at a later stage. TNF- $\alpha$, and NO production was tested at a 2 hour time-point. TNF- $\alpha$ ELISAs and Greiss reactions were run as described in the methods. Treating macrophages with the MSD paclitaxel, peloruside A, or docetaxel did not cause any significant alteration in TNF- $\alpha$ production (Fig 7.2.1A and 7.2.1B). Zampanolide treated macrophages exhibited a trend of decreasing TNF- $\alpha$ 
production with increasing zampanolide dosage (Fig 7.2.1A). Latrunculin A treated macrophages were able to produce TNF- $\alpha$ at levels comparable to those of vehicle control. Additionally treatment with ixabepilone did not alter TNF- $\alpha$ production, while mycothiazole treatment showed a trend of increased TNF- $\alpha$ levels with increasing dose of drug (Fig 7.2.2A and 7.2.2B).

The effect of MSD treatment on NO production was interesting. At high doses of paclitaxel, peloruside A, docetaxel, zampanolide and latrunculin we found inhibition to NO production (Fig 7.2.3). Additionally, while ixabepilone and mycothiazole did not significantly affect NO production (Fig 7.2.3B and 7.2.3C), there was a trend suggesting that high doses of these compounds could affect NO production. 


\subsubsection{Results}

Figure 7.2.1
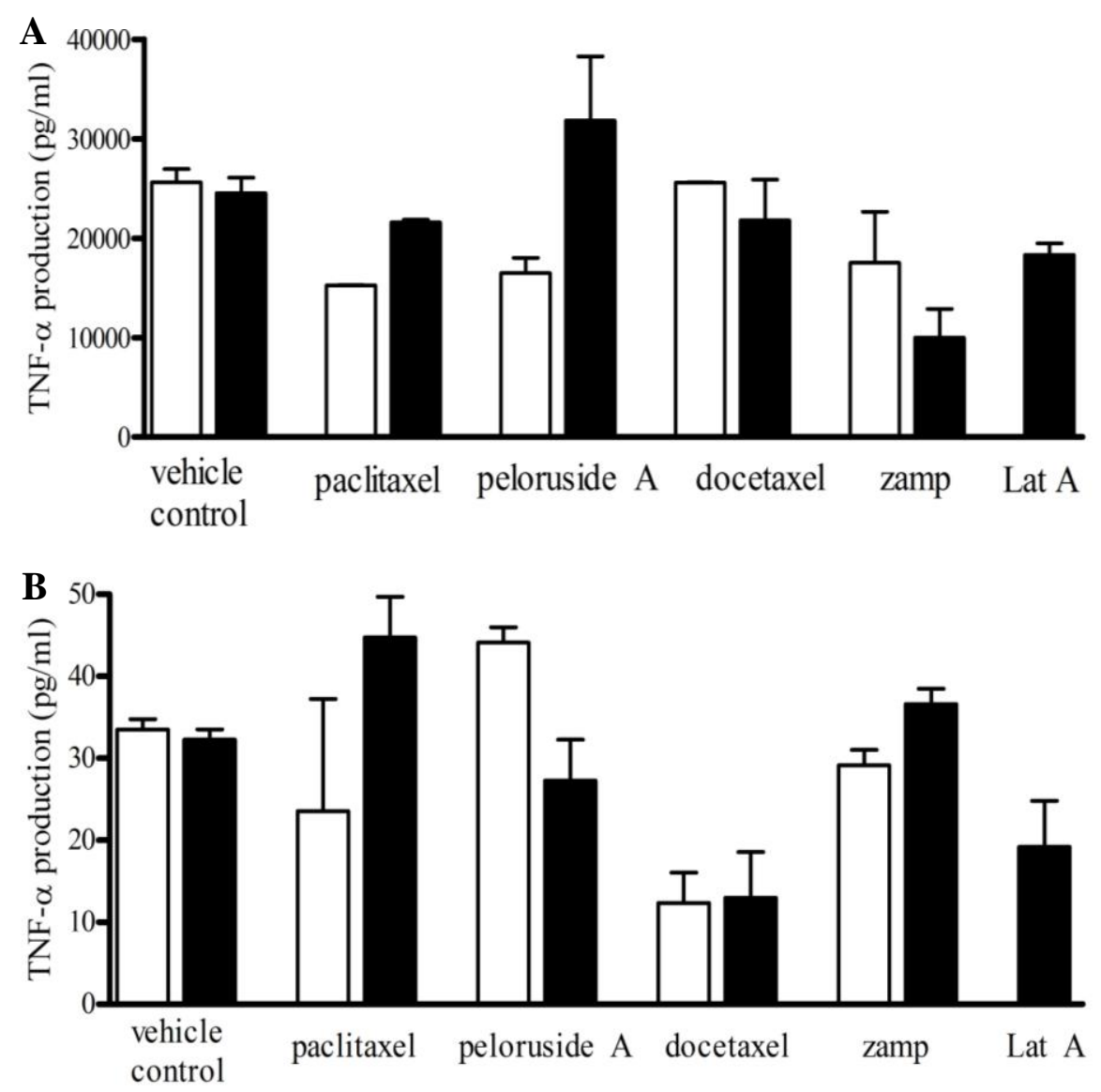

Figure 7.2.1 Drug treatment during pHrodo phagocytosis does not alter TNF-a production in RAW264.7 macrophages. (A, B) Treatment with paclitaxel, peloruside A, docetaxel or zampanolide does not significantly alter TNF- $\alpha$ production during pHrodo phagocytosis. In both graphs, latrunculin A treatment does not inhibit TNF- $\alpha$ production. TNF- $\alpha$ production from $\mathrm{A}$ and $\mathrm{B}$ corresponds to 1 individual pHrodo infection assay each. Graphs are representative of 2 ELISAs each with duplicate wells. p>0.05, one-way ANOVA with Bonferroni post test. White bars indicate $0.1 \mu \mathrm{M}$ (low dose) treatment / vehicle control $\square$, while black bars indicate $1 \mu \mathrm{M}$ (high dose) treatment / vehicle control W. Vehicle controls contain the same final concentration of ethanol as the drug treated samples. Lat A: latrunculin A, zamp: zampanolide. 
Figure 7.2.2
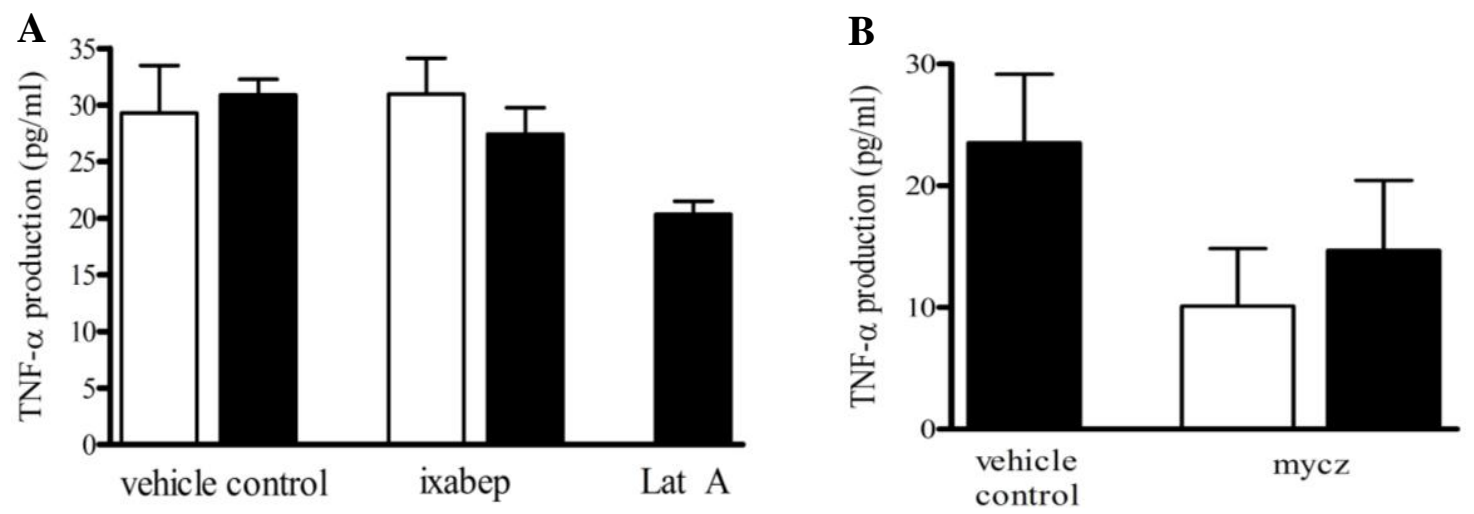

Figure 7.2.2 Drug treatment during pHrodo phagocytosis does not alter TNF-a production in RAW264.7 macrophages. (A) As compared to vehicle control, treatment with ixabepilone does not significantly alter TNF- $\alpha$ production during pHrodo phagocytosis. p>0.05, one-way ANOVA with Bonferroni post test. (B) Treatment with mycothiazole does not alter TNF- $\alpha$ production as compared to DMSO vehicle control. p $>0.05$, one-way ANOVA with Bonferroni post test. White bars indicate $0.1 \mu \mathrm{M}$ (low dose) treatment / vehicle control $\square$, while black bars indicate $1 \mu \mathrm{M}$ (high dose) treatment / vehicle control 1 . Vehicle control samples contain the same final concentration of ethanol or DMSO as the drug treated samples. Lat A: latrunculin A, ixabepilone: ixabep, mycz: mycothiazole. Graphs are representative of 2 experiments, each with duplicate wells. 
Figure 7.2.3
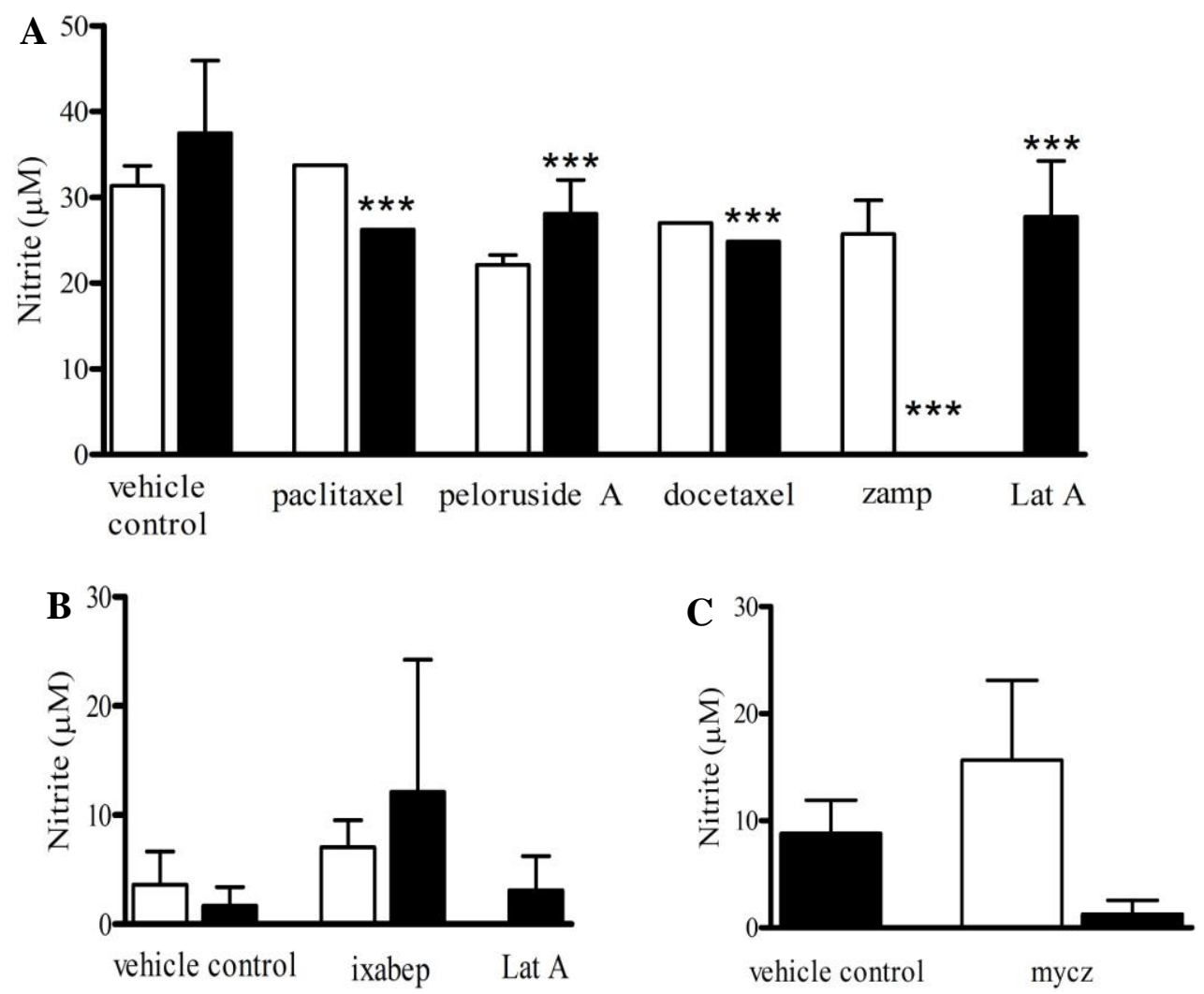

Figure 7.2.3 Treatment with high dose MSD affects nitric oxide production during phagocytosis of bacteria. (A) High doses of paclitaxel, peloruside A, docetaxel zampanolide and latrunculin A significantly inhibit NO production. Additionally, zampanolide treatment eliminates NO production, to a higher degree as compared to other MSD used. ***p<0.001 paclitaxel vs vehicle control, peloruside A vs vehicle control, docetaxel vs vehicle control, zampanolide vs vehicle control, latrunculin A vs vehicle control, one-way ANOVA with Newman-Keuls post test. (B) ixabepilone or (C) mycothiazole treatment does not significantly affect NO production, there is a trend of high doses of both these compounds affecting NO production to a greater degree than low doses of the same compound. p>0.05 one-way ANOVA with Newman-Keuls post test. White bars indicate low dose treatment/vehicle control $\square$, while black bars indicate high dose treatment/vehicle control $\square$. Vehicle control samples contain the same final concentration of ethanol or DMSO as the drug treated samples. Lat A: latrunculin A, zamp: zampanolide, ixabepilone: ixabep, mycz: mycothiazole. Graphs are representative of 2 experiments, each with duplicate wells. 
Chapter 7: Effects of MSD treatment on cytokine production in RAW264.7 macrophages

\subsubsection{Discussion}

This set of assays focussed on the effects of MSD treatment on TNF- $\alpha$ and NO production during pHrodo phagocytosis. Cytokines such as TNF- $\alpha$ and NO stimulate macrophages to increase responses to bacterial invasion (Zhang et al., 2008). Additionally, the presence of TNF- $\alpha$ also helps increase bacterial resistance (Havell, 1989). Researchers have proposed that the pharmacological and toxicological properties of the taxanes could be related in part to the cytokines and pro-inflammatory proteins induced by these actives (Chan et al., 2000; Fitzpatrick et al., 2003). Based on this combined information, we tested infection assay supernatants for the presence inflammatory cytokines TNF- $\alpha$ and NO to investigate the effects that MSD treatment could have on production of these cytokines during phagocytic processes.

We found that MSD treatment did not inhibit TNF- $\alpha$ production during phagocytosis of pHrodo. The levels of TNF- $\alpha$ produced were similar in the presence of paclitaxel, peloruside and docetaxel. We also found that treatment of macrophages with latrunculin A did not inhibit TNF- $\alpha$ production, which indicates that although phagocytosis is impaired due to actin stabilization (Oliveira et al., 1996), LPS and TLR-4 are functional and interact to stimulate an immune response. However, macrophages treated with zampanolide exhibited a trend of decreasing TNF- $\alpha$ production with increasing dose of MSD. This is not surprising, because as seen in chapter 5 and 6 , macrophages treated with zampanolide were unable to phagocytose and/or kill bacteria efficiently. This data taken together suggests that zampanolide treatment could impair immune responses and macrophage function, especially in terms of resistance to bacterial infections. 
Interestingly, as seen with the high and low CFU counts in chapter 6, we observed that when TNF- $\alpha$ production was lower, the trends seen in the presence of MSD treatment were altered to a greater degree than when TNF- $\alpha$ production was higher. We found that paclitaxel treatment consistently exhibited a trend of dose-dependent increase in TNF- $\alpha$ production, irrespective of baseline levels of TNF- $\alpha$. This is most likely due to the LPS mimicry causing higher paclitaxel doses to induce correspondingly higher levels of TNF- $\alpha$ (Ding et al., 1990b). However, we saw contrasting effects with peloruside A treatment. When the baseline levels of TNF- $\alpha$ was high, peloruside A treated macrophages exhibited a trend of dose dependent increase in TNF- $\alpha$ production, but when the baseline level of TNF$\alpha$ was low, peloruside A treatment led to a dose dependent decrease in TNF- $\alpha$ production. This is surprising as peloruside A lacks LPS mimicry and displays an anti-inflammatory profile in LPS-stimulated BMMO (Crume et al., 2007). A possible reason for this could be that with high levels of TNF- $\alpha$ present, peloruside A was unable to exert its antiinflammatory profile. This is beneficial in terms of clinical use, as if bacterial load is high, macrophages are stimulated to increase inflammatory cytokines and ameliorate infection, and peloruside A treatment would not inhibit macrophage response to bacteria.

We saw a similar effect with docetaxel treated macrophages. Docetaxel does not have LPS mimicry or display an inflammatory profile (Manthey et al., 1993; Fitzpatrick et al., 2003). When TNF- $\alpha$ levels were low, docetaxel caused an inhibition in TNF- $\alpha$ production, but this was not significant as compared to vehicle treated macrophages. However when TNF- $\alpha$ levels were higher, docetaxel did not inhibit TNF- $\alpha$ production. We also looked at the effects of ixabepilone and mycothiazole treatment on TNF- $\alpha$ production, and found no 
Chapter 7: Effects of MSD treatment on cytokine production in RAW264.7 macrophages

significant changes to TNF- $\alpha$ production in the presence of high or low doses of these two compounds.

Results from NO production were most interesting in terms of alterations to cytokine production. We measured $\mathrm{NO}_{2}^{-}$production as an indicator of $\mathrm{NO}$ production as described in the methods. Our study measures NO production as at the 2 hour time point, however optimal production of NO occurs at later time points and production is normally measured at 8 hours (Robinson, 2009), 20 hours (Nakano et al., 1999), 24 hours (Stuehr et al., 1985; Sosroseno et al., 2002), 48 hours (Kirikae et al., 1996) or even as late as 72 hours (Crume et al., 2007). While these studies measured optimal peak levels of NO production, there are studies that have shown low levels of NO being produced in the presence of LPS, at earlier time points of 2 or 4 hours (Chamulitrat et al., 1995; Xie et al., 1995), which is similar to our results, where NO was detected at 2 and 3 hour time points.

We found that high doses of paclitaxel, peloruside A, docetaxel, zampanolide and latrunculin A caused significant inhibition to NO production during pHrodo phagocytosis. While the data is significant, it is preliminary and it would be worthwhile to repeat these assays for a greater understanding of the inhibition seen with high doses of MSD on NO production. Interestingly, paclitaxel, peloruside A, docetaxel and latrunculin A inhibited NO; however, high dose zampanolide treatment completely blocked NO production. This suggests that zampanolide could possibly be used as an anti-inflammatory therapeutic similarly to peloruside A, as we found zampanolide inhibited TNF- $\alpha$ production as well as NO production. Since the data suggests that MSD inhibits NO production, it would be valuable to study the effects of not only zampanolide, but also the other MSD on NO 
production at later time points. This would enable understanding MSD effects when optimal NO is produced during phagocytosis.

While ixabepilone and mycothiazole did not significantly alter NO production, there was a trend suggesting high dose mycothiazole reduced NO production similarly to zampanolide. Results from ixabepilone are variable and it is hard to conclude or predict the possible effects of this drug on NO production. Overall these results are preliminary and it would be worthwhile to repeat these assays for a better understanding of the significance of the data.

Overall, we found that MSD treatment can considerably alter TNF- $\alpha$ and NO production. Interesting effects were seen with peloruside A in terms of reduction of TNF- $\alpha$ production, which would be useful when developing this compound for clinical use. We also found zampanolide treatment significantly inhibited cytokine production. Taken together, these results suggest that MSD treatment significantly alters the levels of cytokine production during bacterial infections. Furthermore, the effects of MSD on phagocytic efficiency may be unrelated to the microtubule stabilizing capabilities of MSD and could likely be due to MSD altering cytokine production, which in turn affects phagocytosis, as suggested previously (Chan et al., 2000; Fitzpatrick et al., 2003). 
Chapter 7: Effects of MSD treatment on cytokine production in RAW264.7 macrophages

\subsection{Changes in cytokine production during bacterial killing in MSD treated} macrophages.

\subsubsection{Introduction}

This set of assays focussed on the effects of MSD on cytokine production during bacterial killing. As described in section 7.2, MSD treatment can affect cytokine production, which directly affects macrophage ability to function. TNF- $\alpha$, and NO are important in maintanence of bacterial resistance (Havell, 1989; Bogdan et al., 2000). Additionally, IL-12 is also produced by bacterial or LPS stimulated macrophages and this cytokine regulates activation of the adaptive immune response (Trinchieri, 2003; Hamza et al., 2010). Similarly to cytokine detection during bacterial phagocytosis, we investigated the effects of MSD treatment on the production of TNF- $\alpha$, NO and Il-12 during bacterial killing. We expected to find high levels of TNF- $\alpha$, as peak levels of this cytokine are produced early on. However, we expected low or limited production of NO and IL-12 as these cytokines are typically produced later in the immune response, once TNF- peaks and begins to decline. This is evidenced by production of NO and IL-12 being typically measured at later time points (Stuehr et al., 1987; Mullins et al., 1999; Nakano et al., 1999).

As described in the methods, supernatants were collected during bacterial killing assays with E.coli, and stored at $-20^{\circ} \mathrm{C}$ for cytokine analysis at a later stage. TNF- $\alpha$, NO and IL-12 production was tested as at a 3 hour hour time-point. As described with high and low $\mathrm{CFU}$ results in chapter $6, \mathrm{TNF}-\alpha$ production is presented corresponding to high or low CFU values, which is noted in figure legends. TNF- $\alpha$ and IL-12 ELISAs and Greiss reactions were run as described in the methods. Compared to vehicle controls, peloruside A 
and docetaxel did not significantly increase or decrease TNF- $\alpha$ production, irrespective of the presence of high or low CFU counts. (Fig 7.3.1A and B) With paclitaxel, at the low CFU counts (Fig 7.3.1B), we found an increase in TNF- $\alpha$ production, but there was no corresponding increase in TNF- $\alpha$ at the high dose of paclitaxel. Interestingly, with low dose zampanolide (Fig 7.3.2A and B) TNF- $\alpha$ production was significantly reduced when CFU counts were higher as compared to when CFU counts were low. TNF- $\alpha$ production was not altered in macrophages treated with ixabepilone or mycothiazole (Fig 7.3.2C) 


\subsubsection{Results}

Figure 7.3.1
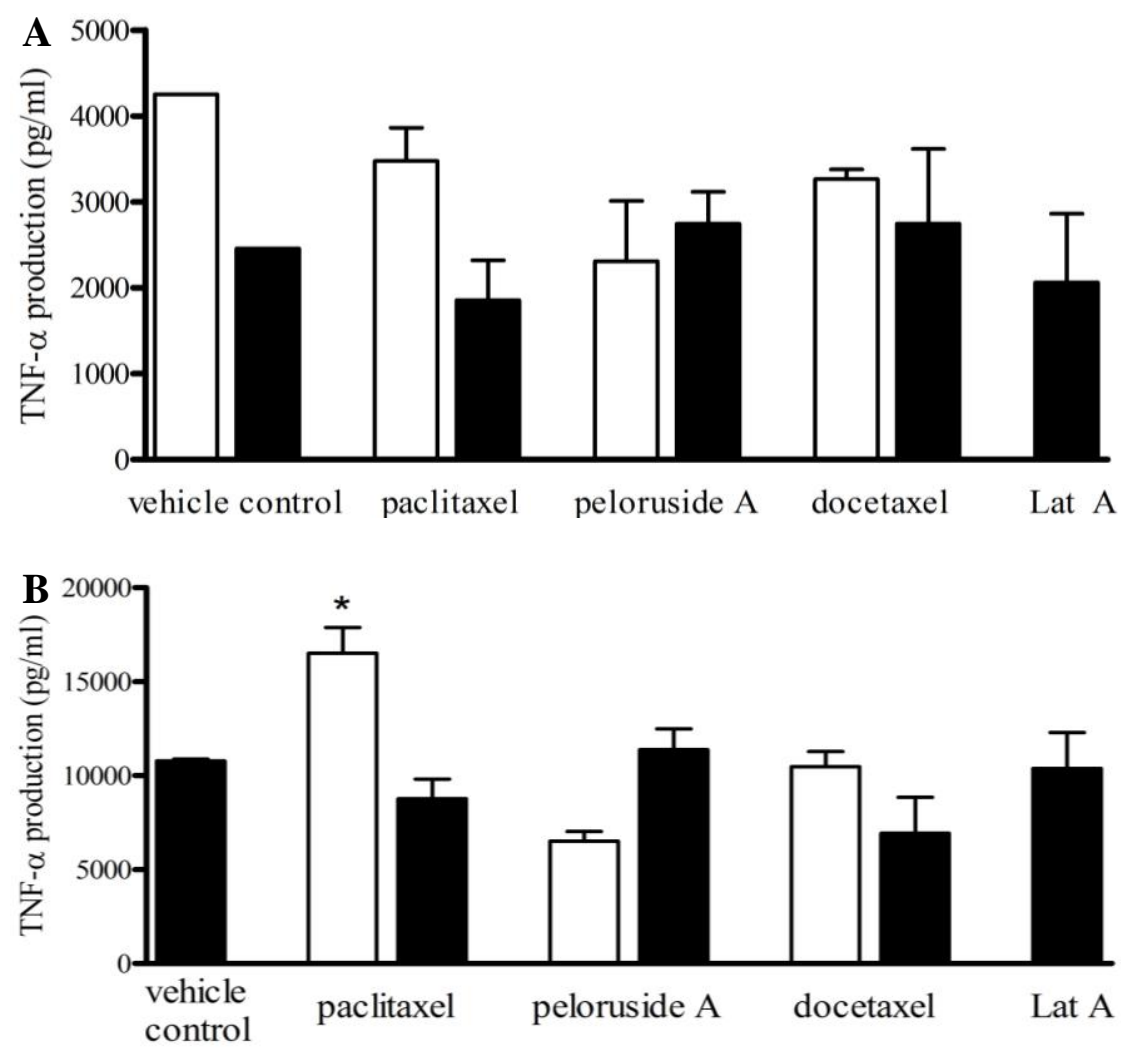

Figure 7.3.1 Changes in TNF- $\alpha$ production by MSD treated macrophages during bacterial killing. (A) TNF- $\alpha$ production corresponds to high CFU counts. There is no significant alteration in TNF- $\alpha$ production when treated with MSD. (B) TNF- $\alpha$ production corresponds to low CFU counts. Compared to vehicle controls, low dose paclitaxel significantly increases TNF- $\alpha$ production. ${ }^{*} \mathrm{p}<0.05$ low dose paclitaxel vs vehicle control, one-way ANOVA with Newman-Keuls post test. TNF- $\alpha$ production from A and B corresponds to 1 individual infection assay each. Graphs are representative of 1 ELISAs each with duplicate wells. Latrunculin A does not inhibit TNF- $\alpha$ production. White bars indicate low dose treatment/vehicle control $\square$, while black bars indicate high dose treatment/vehicle control $\square$. Vehicle controls contain the same final concentration of ethanol as the drug treated samples. Lat A: latrunculin A. 
Figure 7.3.2
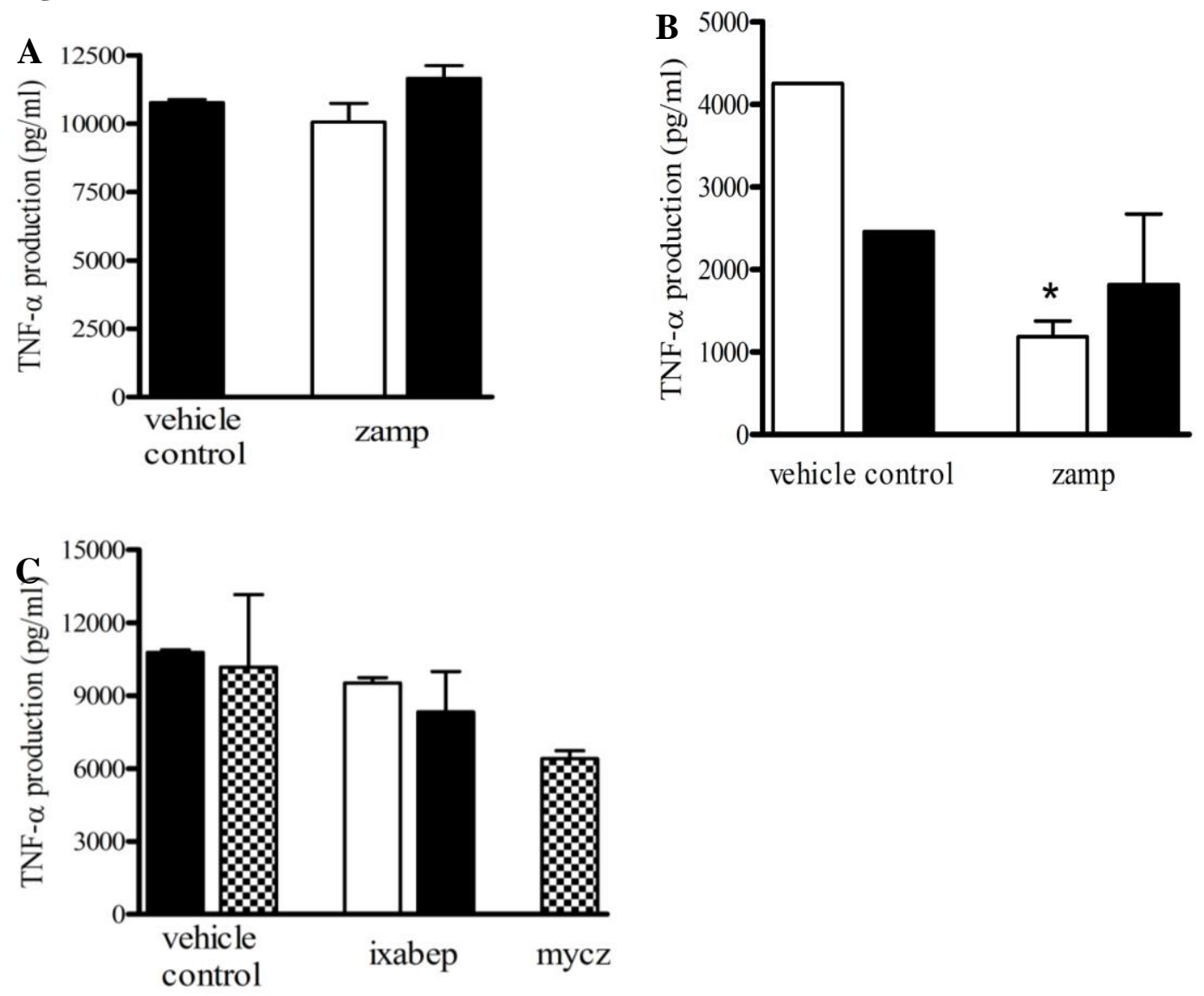

Figure 7.3.2 Effect of zampanolide, ixabepilone and mycothiazole on TNF-a production during bacterial killing. (A) TNF- $\alpha$ production corresponds to low CFU counts. There is no significant alteration in TNF- $\alpha$ production when treated with zampanolide. p>0.05, one-way ANOVA with Newman-Keuls post test. (B) TNF- $\alpha$ production corresponds to high CFU counts. Compared to vehicle controls, low dose zampanolide significantly decreases TNF- $\alpha$ production. ${ }^{*} \mathrm{p}<0.05$ low dose zampanolide vs vehicle control, one-way ANOVA with Newman-Keuls post test. (C) TNF- $\alpha$ production corresponds to low CFU counts. There is no significant alteration in TNF- $\alpha$ production when treated with ixabepilone or mycothiazole. $\mathrm{p}>0.05$, one-way ANOVA with NewmanKeuls post test. Graphs are representative of 1 ELISA each with duplicate wells. White bars indicate low dose treatment/vehicle control $\square$, while black bars indicate high dose treatment/vehicle control $\square$, chequered bars indicate $1 \mu \mathrm{M}$ mycothiazole and its DMSO vehicle control $\mathbf{H}$. Vehicle controls contain the same final concentration of ethanol or DMSO as the drug treated samples. Lat A: latrunculin A, zamp: zampanolide, ixabep: ixabepilone, mycz: mycothiazole. 
Figure 7.3.3
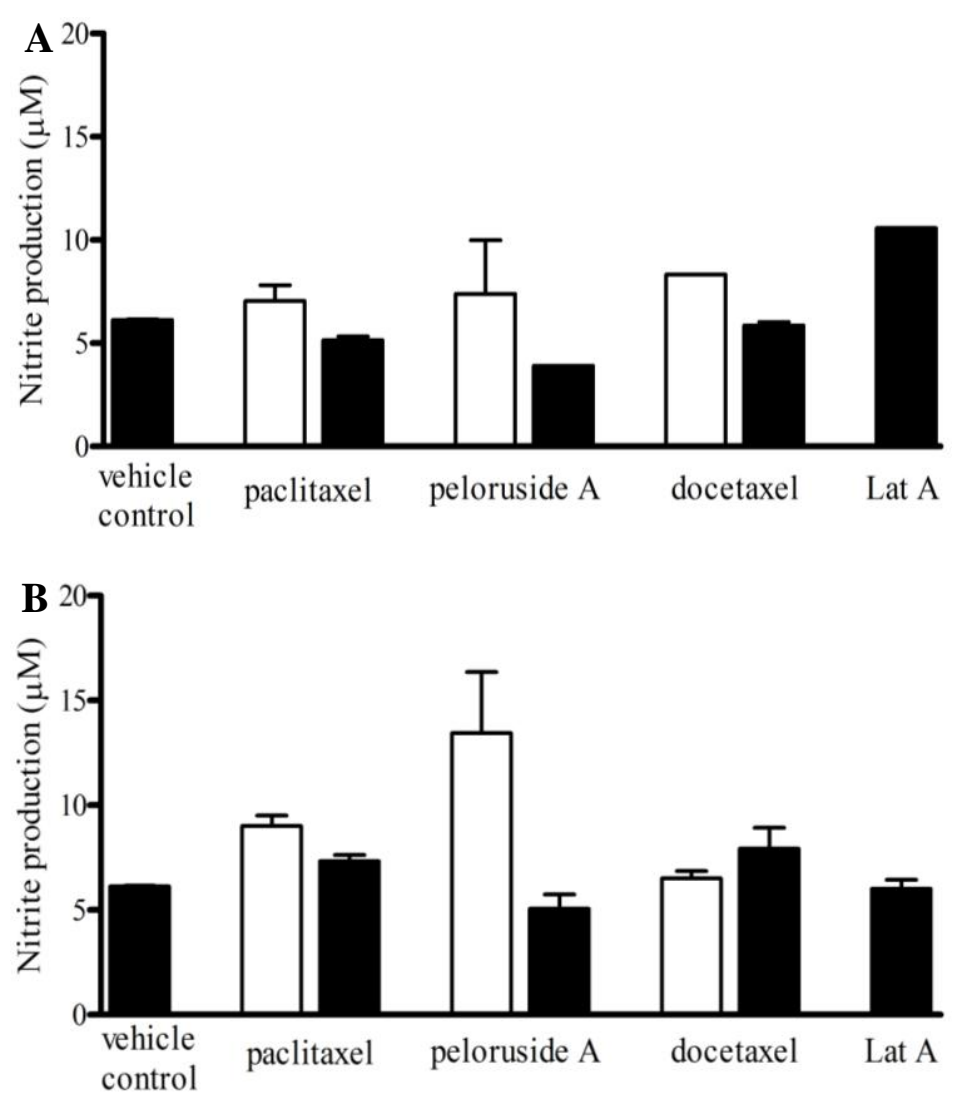

Figure 7.3.3 NO production is not significantly affected by MSD treatment at (A) 1 hour or (B) 3 hours during bacterial killing. (A) There was no significant alteration to NO production during bacterial killing in the presence of paclitaxel, peloruside A, docetaxel or latrunculin $A$ at 1 hour of drug treatment. p>0.05, one-way ANOVA with Newman-Keuls post test (B) There was no significant alteration to NO production during bacterial killing in the presence of paclitaxel, peloruside A, docetaxel or latrunculin A at 3 hours of drug treatment. p>0.05, one-way ANOVA with Newman-Keuls post test For both graphs, NO production corresponds to low CFU counts. White bars indicate $0.1 \mu \mathrm{M}$ (low dose) treatment / vehicle control $\square$, while black bars indicate $1 \mu \mathrm{M}$ (high dose) treatment / vehicle control Vehicle controls contain the same final concentration of ethanol as the drug treated samples. Lat A: latrunculin A 
Figure 7.3.4
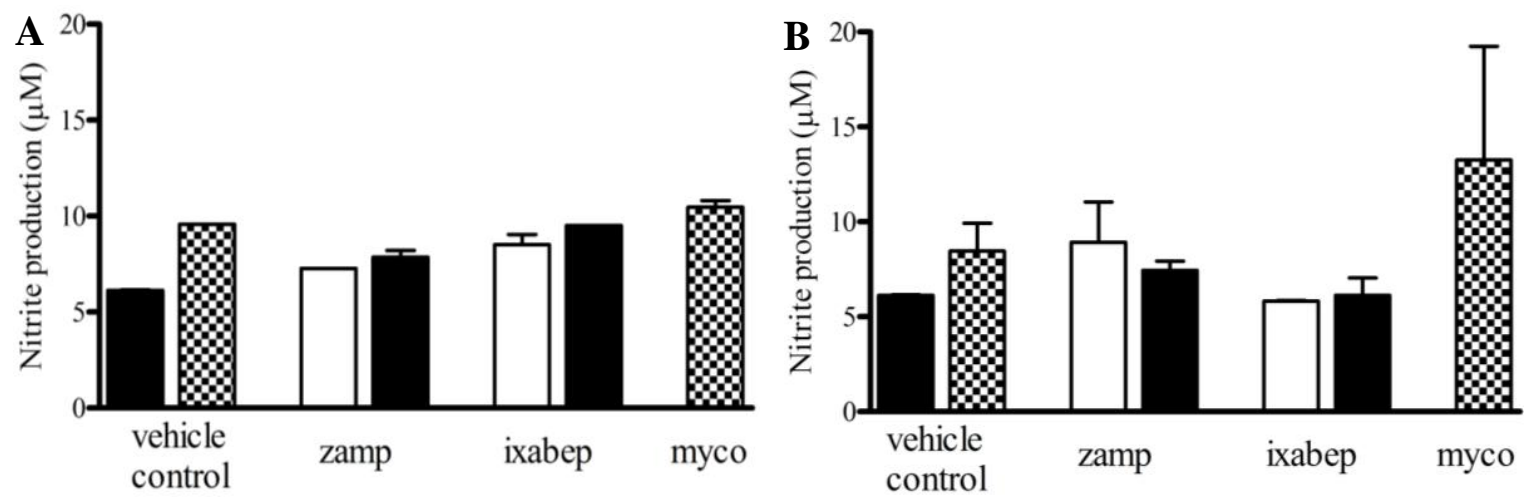

Figure 7.3.4 NO production is not significantly affected by MSD or mycothiazole treatment at (A) 1 hour or (B) 3 hours during bacterial killing. (A) There was no significant alteration to NO production during bacterial killing in the presence of zampanolide, ixabepilone or mycothiazole at 1 hour of drug treatment. p $>0.05$, one-way ANOVA with Newman-Keuls post test (B) There was no significant alteration to NO production during bacterial killing in the presence of zampanolide, ixabepilone or mycothiazole at 3 hours of drug treatment. $p>0.05$, one-way ANOVA with Newman-Keuls post test For both graphs, NO production corresponds to low CFU counts. White bars indicate low dose MSD treatment $\square$, while black bars indicate high dose treatment/vehicle control $\square$, chequered bars indicate $1 \mu \mathrm{M}$ mycothiazole and its DMSO vehicle control $\mathbf{H}$. Vehicle controls contain the same final concentration of ethanol or DMSO as the drug treated samples. Lat A: latrunculin A, zamp: zampanolide, ixabep: ixabepilone, mycz: mycothiazole. 
Figure 7.3.5

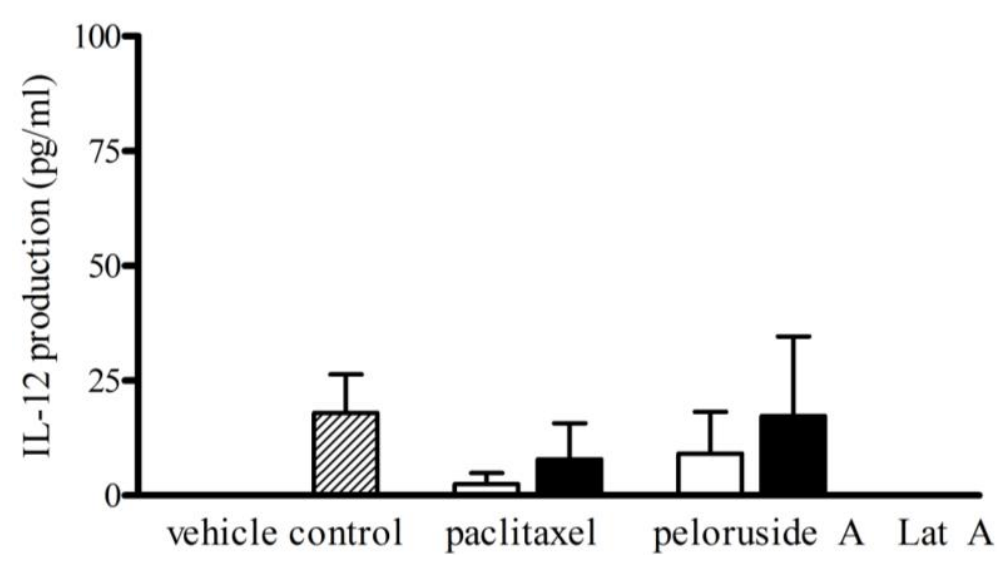

Figure 7.3.5 At 3 hours, Il-12 production is variable during bacterial killing in MSD treated macrophages. IL-12 production corresponds to high CFU counts. There is no significant change in level of IL-12 produced when treated with paclitaxel, peloruside A, or latrunculin $\mathrm{A}$ at 3 hours. p>0.05 one-way ANOVA with Bonferroni post test. White bars indicate $0.1 \mu \mathrm{M}$ (low dose) MSD treatment $\square$, while black bars indicate $1 \mu \mathrm{M}$ (high dose) treatment / vehicle control $\square$, lined bars $\mathscr{Z}$ indicate macrophages with bacteria, used instead of vehicle controls, which did not produce measureable IL-12. Vehicle controls contain the same final concentration of ethanol as the drug treated samples. Lat A: latrunculin A. 
Chapter 7: Effects of MSD treatment on cytokine production in RAW264.7 macrophages

\subsubsection{Discussion}

The main aim of these assays was studying the role of cytokines in bacterial killing and the changes that occurred following MSD treatment. As described previously, the immunomodulation seen in the presence of MSD such as paclitaxel and docetaxel or peloruside A could be well be due to the effects of these compounds on cytokine production (Chan et al., 2000), rather than a direct effect of MSD on processes such as bacterial killing. To better understand the results we saw during bacterial killing by MSD treated macrophages, we studied the cytokines produced during these events. TNF- $\alpha$ was an obvious cytokine to study due to its role in bacterial defense (Havell, 1989), and its signaling via TLR-4 to activate macrophages (Tracey et al., 1994). TNF- $\alpha$ is also one of the earlier cytokines produced during bacterial infections (Fong et al., 1989; Tracey et al., 1993). Besides TNF- $\alpha$, we studied changes to levels of NO and IL-12 as these cytokines are also produced during bacterial infections (Holan et al., 2001; Hamza et al., 2010). While TNF- $\alpha$ is produced within 90 minutes of bacterial stimulus (Fong et al., 1989), NO and IL12 are typically produced later on in the infection process and generally peak in production once TNF- $\alpha$ production begins to decline (Stuehr et al., 1987; Mullins et al., 1999). However we hypothesized that with the excess LPS and bacterial stimulation there may be low levels of NO and IL-12 produced at the earlier time points.

We found that levels of TNF- $\alpha$ produced fell into two categories of high and low cytokine production. Not surprisingly, the high and low cytokine levels corresponded to the high and low CFU counts in the bacterial killing assays in chapter 6 . We found that when CFU counts were higher, levels of TNF- $\alpha$ increased, and the various MSD treatments had no significant effect on modifying TNF- $\alpha$ production. Interestingly when TNF- $\alpha$ levels and 
CFU counts were low, paclitaxel treatment at low doses caused a significant increase in levels of TNF- $\alpha$ produced. This increase corresponded to a significant increase in bacterial survival, as seen in chapter 6 (Fig 6.2.1), where macrophage bactericidal ability was impaired. While TNF- $\alpha$ levels are high, it is possible that the higher levels of LPS in combination with paclitaxel stimulation caused internalization of TNFR (Ding et al., 1989; Ding et al., 1990b). Thus although TNF- $\alpha$ is produced, it maybe unable to exert bactericidal effects due to lack of cellular receptors and this could have caused the increased CFU seen. We did not find any significant alteration to $\mathrm{TNF}-\alpha$ production in peloruside A or docetaxel treated macrophages. This corresponds to CFU data where there was no significant alteration to bacterial survival in the presence of these two MSDs.

Treatment with zampanolide, ixabepilone and mycothiazole did not alter TNF- $\alpha$ production when there was high cytokine production. This is interesting as the high cytokine data corresponds to the low CFU data in chapter 6 (Fig 6.2.2). When CFU counts were low, ixabepilone treated macrophages showed significantly increased bactericidal activity. Ixabepilone stabilizes microtubules similarly to paclitaxel and it is a competitive inhibitor for the binding site of paclitaxel (Bollag et al., 1995; Goodin, 2008). Additionally as noted in previous sections, ixabepilone is effective in paclitaxel resistant cell lines (Bollag et al., 1995; Conlin et al., 2007). This suggests that the two MSD are similar, however, ixabepilone LPS mimicry status is unknown. It would be worth investigating this, as having LPS mimicry would cause internalization of TNFR (Ding et al., 1989), but lack of LPS mimicry would mean that any TNF- $\alpha$ produced would be utilized for bacterial killing. We found that mycothiazole treated cells had impaired bacterial killing, while still producing relatively high levels of TNF- $\alpha$. pHrodo phagocytosis data suggests that 
mycothiazole treatment does not impair phagocytic capability. The data suggests that while mycothiazole treatment does not alter initial TLR stimulation via LPS or cytokine production, signaling pathways and trafficking or transport processes involved in killing may be impaired. However, more in-depth studies are needed before we can speculate on the mechanism by which mycothiazole affects bacterial killing.

The effects of zampanolide on TNF- $\alpha$ production was also remarkable. In chapter 6 , our data suggested that in the presence of zampanolide, there is limited phagocytosis, and bacteria that are taken up cannot be killed. The TFN- $\alpha$ data in figure 7.3.2A and B corresponds to low and high CFU counts, respectively. When CFU counts are high, there is no alteration to TNF- $\alpha$ production and the cytokine is produced in amounts similar to that of vehicle treated cells. In contrast, in the presence of low CFU counts, cytokine production is significantly decreased. Together the data suggests that in the presence of sufficient LPS stimuli, cytokine production is maintained, but the drug treatment blocks the bactericidal effect of the cytokine. However, in the presence of low LPS stimulation, zampanolide is able to alter cytokine production and thus inhibit bacterial phagocytosis. It was recently found that release of TNF- $\alpha$ in macrophages occurs via recycling endosomes and that the phagosome plays a role in delivery of this endosome to the cellular surface (Murray et al., 2005; Manderson et al., 2007). It is possible that zampanolide treatment affects membrane trafficking and thus inhibits phagocytosis and cytokine release via affecting function of the recycling endosomes. Microtubules could also be affected thus further inhibiting antigen processing (Peachman et al., 2004).

We looked at NO production at 1 and 3 hours during bacterial killing. While there were low levels of NO produced from all samples, there were no significant effects of MSD 
treatment on NO production. Part of this could be related to the fact that these timepoints are early and less $\mathrm{NO}$ is produced within this time frame, with the result that we do not see any alteration in NO levels. However, despite this being preliminary data with low levels of NO production, there are interesting trends in the presence of peloruside A treated macrophages. Compared to the 1 hour time point, NO production increases by 3 hours. In paclitaxel treated samples, there is no significant reduction in NO production between 1 and 3 hours. However at 3 hours, in the presence of low dose peloruside A treated samples, NO production increases, but at the higher dose, peloruside A seems to lower NO production to levels similar to those seen at 1 hour. This is similar to results seen previously where peloruside A decreases inflammatory cytokines (Crume et al., 2007). It would be extremely interesting to study IFN- $\gamma$ and IL-12 along with NO cytokine production in macrophages treated with MSD for longer periods. Considering that LPS stimulus is high due to presence of bacteria, the NO levels measured were comparatively low; once again suggesting that it would be advisable to measure this cytokine at later time points. We also studied IL-12 production; however this cytokine is also normally measured at later time points. The data is extremely variable and it is hard to speculate on any MSD related effects or trends exhibited. It is difficult to comment on IL-12 production as vehicle treated cells did not produce IL-12. While it is possible that ethanol vehicle control played a role in decreasing levels of IL-12, it would be advisable and more accurate to measure IL-12 production at later time points during bacterial phagocytosis and killing. There are studies that report NO and IL-12 production is linked, where IL-12 increases NO production in the presence of LPS (Sosroseno et al., 2002) and IL-12 gene expression is regulated by NO (Rothe et al., 1996). In view of these studies, it would be advisable to study IL-12 and NO in conjunction 
and at later time points. Additionally, when studying NO production in the presence of MSD, it would be interesting to study phagocytosis and killing in primary macrophages, as these cells do not proliferate and drug treatments can be studied over longer periods of time.

Overall, we found that MSD treatment affects cytokine production although these effects might not translate into inhibition or enhancement during bacterial killing. We also found that $\mathrm{TNF}-\alpha$ production was altered with zampanolide treatment when bacterial killing was un-affected, thus suggesting that zampanolide can affect cytokine production distinctively to macrophage function. The data suggests that paclitaxel, peloruside A and docetaxel treatment do not affect TNF- $\alpha$ production. We also found that in limiting LPS conditions paclitaxel does not decrease TNF- $\alpha$ production but via its LPS mimicry, TNF- $\alpha$ production is increased or maintained at normal levels. We found that increasing inflammatory stimuli in RAW264.7 macrophages did not cause an increase in cell metabolism due to the high sensitivity of this cell line to LPS. Finally, it would be worth repeating TNF- $\alpha$ and NO tests as these results are from one or two experiments and more valuable conclusions can be drawn with a bigger sample size. 
Chapter 7: Effects of MSD treatment on cytokine production in RAW264.7 macrophages

\subsection{Effect of IFN- $\gamma$ pretreatment on TNF- $\alpha$ production in MSD treated} macrophages during phagocytosis and killing.

\subsubsection{Introduction}

This set of assays examined the effect of IFN- $\gamma$ pre-treatment on TNF- $\alpha$ production during bacterial phagocytosis and killing in MSD treated macrophages. We were interested in studying any enhancement or inhibition to levels of TNF- $\alpha$ and linking this to the absence or presence of IFN- $\gamma$ pre-treatment. IFN- $\gamma$ stimulates macrophages and induces antimicrobial mechanisms, along with up-regulating antigen processing and presentation pathways (Schroder et al., 2004). INF- $\gamma$ also has a role in regulation of immunomodulatory cytokines TNF- $\alpha$ and IL-12 (Bach et al., 1997). Due to this, pre-treating macrophages with IFN- $\gamma$ caused optimal activation and enabled macrophages to efficiently phagocytose and kill bacteria. However we were interested in the effects of MSD, particularly when optimal states of activation were absent. We wanted to understand if MSD treatment during suboptimal activation states would enhance or further impair macrophage bactericidal activity. This was interesting since paclitaxel has LPS mimicry (Ding et al., 1990b), while paclitaxel and docetaxel are immunomodulatory (Chan et al., 2000).

Figure 7.4.1 shows effects of IFN- $\gamma$ pre-treatment on TNF- $\alpha$ production during bacterial killing in the presence of MSD treatment. Overall, comparing the effects of IFN- $\gamma$ treatment, higher levels of TNF- $\alpha$ were produced in the presence of IFN- $\gamma$, irrespective of MSD treatment, which was as expected (Fig 7.4.1A). In the absence of IFN- $\gamma$ pretreatment, MSD treatment seems to stimulate TNF- $\alpha$ production (Fig 7.4.1B). However MSD treatment did not increase TNF- $\alpha$ production to levels seen with IFN- $\gamma$ pre-treatment. We found that in the presence of IFN- $\gamma$, treatment with high dose docetaxel, latrunculin A 
or colchicine caused a significant decrease in TNF- $\alpha$ production. In the absence of IFN- $\gamma$, low dose paclitaxel treatment significantly increased TNF- $\alpha$ production, while colchicine significantly decreased TNF- $\alpha$ production. Figure 7.4.2 shows effects of IFN- $\gamma$ pretreatment on TNF- $\alpha$ production during pHrodo phagocytosis in the presence of MSD treatment. There was an increase in TNF- $\alpha$ production in the presence of low dose paclitaxel and colchicine treated macrophages once again showed a significant decrease in TNF- $\alpha$ production. 
Figure 7.4.1
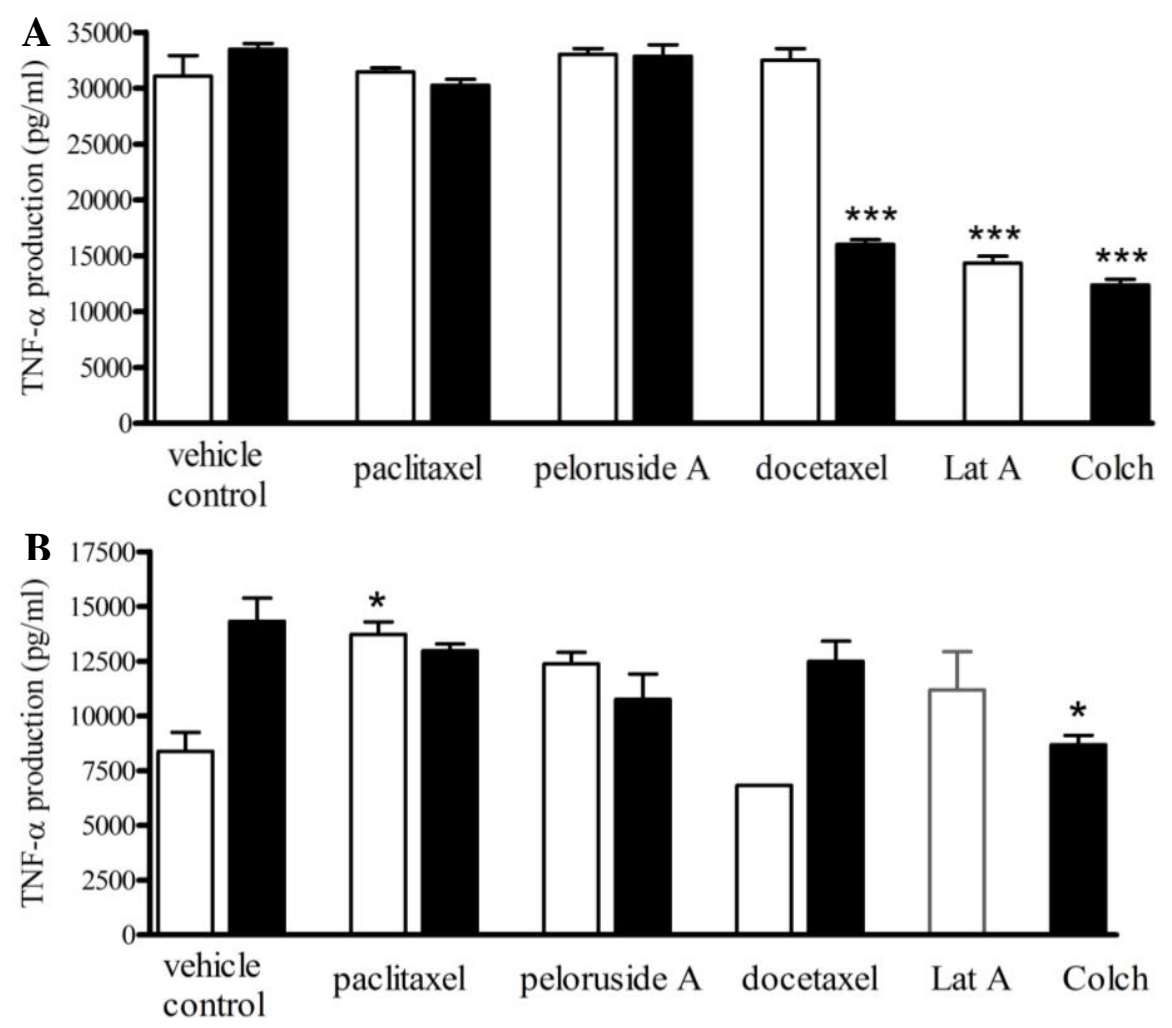

Figure 7.4.1 Absence or presence of IFN- $\gamma$ pre-treatment alters TNF- $\alpha$ production during bacterial killing in drug treated RAW264.7 macrophages. (A) RAW264.7 macrophages are pre-treated with IFN- $\gamma$. TNF- $\alpha$ production is significantly reduced in the presence of high dose docetaxel, latrunculin A and colchicine when macrophages are pretreated with IFN- $\gamma$. *** $p<0.001$ docetaxel vs vehicle control, latrunculin A vs vehicle control, colchicine vs vehicle control, one-way ANOVA with Newman-Keuls post test. (B) RAW264.7 macrophages lack IFN- $\gamma$ pre-treatment. TNF- $\alpha$ production is significantly increased in the presence of low dose paclitaxel and reduced in the presence of colchicine when IFN- $\gamma$ pre-treatment is absent. ${ }^{*} \mathrm{p}<0.05$ paclitaxel vs vehicle control, colchicine vs vehicle control, one-way ANOVA with Newman-Keuls post test. Both graphs are representative of 1 experiment, each with duplicate wells. White bars indicate low dose MSD treatment $\square$, while black bars indicate high dose treatment/vehicle control $\square$. Vehicle controls contain the same final concentration of ethanol or DMSO as the drug treated samples. Lat A: latrunculin A, colch: colchicine 
Figure 7.4.2
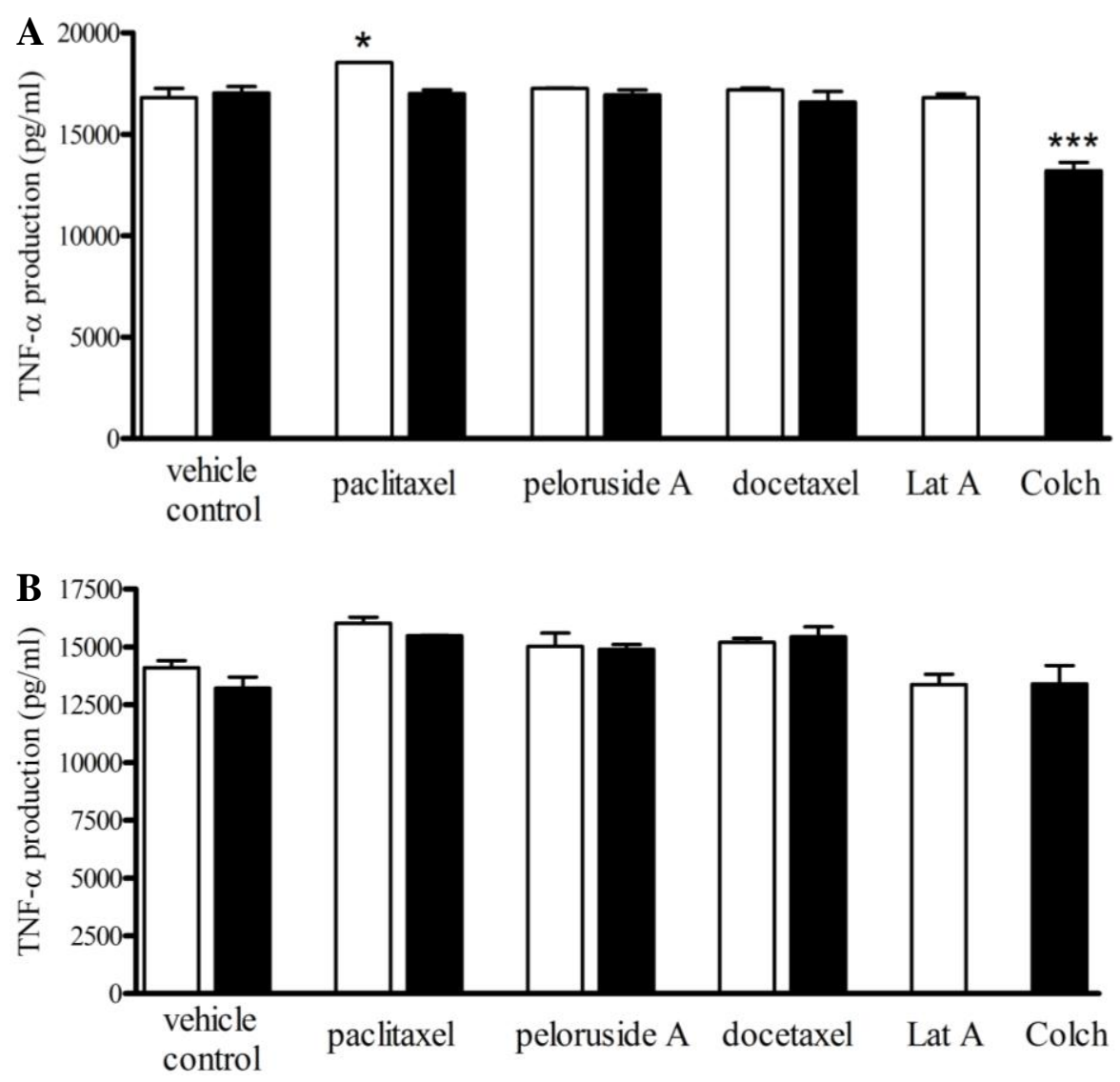

Figure 7.4.2 Absence or presence of IFN- $\gamma$ pre-treatment alters TNF- $\alpha$ production during phagocytosis in drug treated RAW264.7 macrophages. (A) RAW264.7 macrophages are pre-treated with IFN- $\gamma$. TNF- $\alpha$ production is significantly increased in the presence of low dose paclitaxel and reduced in the presence of colchicine when macrophages are pre-treated with IFN- $\gamma$. ${ }^{*} \mathrm{p}<0.05$ paclitaxel vs vehicle control, $* * * \mathrm{p}<0.001$ colchicine vs vehicle control, one-way ANOVA with Newman-Keuls post test. (B) RAW264.7 macrophages lack IFN- $\gamma$ pre-treatment. TNF- $\alpha$ production is not altered when IFN- $\gamma$ pre-treatment is absent. $p>0.05$, one-way ANOVA with Newman-Keuls post test. Both graphs are representative of 1 experiment, each with duplicate wells. White bars indicate low dose MSD treatment/vehicle control $\square$, while black bars indicate high dose treatment/vehicle control Vehicle controls contain the same final concentration of ethanol or DMSO as the drug treated samples. Lat A: latrunculin A, colch: colchicine 
Chapter 7: Effects of MSD treatment on cytokine production in RAW264.7 macrophages

\subsubsection{Discussion}

These assays were carried out to study the effects of MSD treatments on bacterial phagocytosis and killing, when optimal stimulation was absent. To test this, we carried out these assays in the absence and presence of IFN- $\gamma$. As described earlier, IFN- $\gamma$ activates macrophages to display microbicidal functions, and secrete pro-inflammatory cytokines (Young et al., 1995; Schroder et al., 2004). However, in previous assays we pre-treated macrophages with IFN- $\gamma$ to activate efficient responses to bacterial challenge and this could have masked MSD related effects. It has also been proposed that MSD treatment may affect cytokines which in turn affect macrophage function (Chan et al., 2000).

We investigated the effects of IFN- $\gamma$ on TNF- $\alpha$ production during bacterial killing. Presence of IFN- $\gamma$ led to increased TNF- $\alpha$ production as macrophages were activated and bactericidal responses were optimal (Schroder et al., 2004). However in the absence of IFN- $\gamma$, lower levels of TNF- $\alpha$ were produced due to the lack of stimulatory signals, despite the presence of LPS. As compared to levels of TNF- $\alpha$ during bacterial killing, levels of TNF- $\alpha$ were lower during bacterial phagocytosis (Fig 7.4.2), irrespective of the presence of IFN- $\gamma$. This indicated that the role of IFN- $\gamma$ was primarily to activate macrophages during bacterial killing, while phagocytosis occurred via FcR, CRs and TLR-4 signalling. This data also suggests that TNF- $\alpha$ production is associated with bactericidal effects to a greater extent as compared to phagocytic events (Havell, 1989).

When studying bacterial killing, in the presence of IFN- $\gamma$, we found no change to TNF- $\alpha$ production when macrophages were treated with paclitaxel or peloruside A. This was surprising in the case of paclitaxel treatment, as paclitaxel mimics LPS and we expected increases in production of inflammatory cytokines such as TNF- $\alpha$. However, it is 
possible that the presence of IFN- $\gamma$ and LPS led to maximal TNF- $\alpha$ production and additional stimulation with paclitaxel did not result in excess TNF- $\alpha$ being produced. In contrast during bacterial phagocytosis, levels of TNF- $\alpha$ were lower irrespective of the presence of IFN- $\gamma$. In these conditions, low dose paclitaxel caused a small but significant increase in TNF- $\alpha$ production. However with increasing dose of paclitaxel, there was no increase in TNF- $\alpha$ production, possibly due to the higher dose MSD treatment impairing delivery of TNF- $\alpha$ via recycling endosomes (Murray et al., 2005; Manderson et al., 2007). Treatment of macrophages with peloruside A did not cause any alterations to TNF- $\alpha$ production during bacterial phagocytosis or killing, irrespective of the absence or presence of IFN- $\gamma$. This was surprising as peloruside $\mathrm{A}$ is known to decrease pro-inflammatory mediators (Crume et al., 2007). However we did not see this decrease even when levels of TNF- $\alpha$ were high as in the presence of IFN- $\gamma$. In contrast, treatment with docetaxel at high dose caused a significant decrease in TNF- $\alpha$ production during bacterial killing. It is known that docetaxel has immunomodulatory properties (Chan et al., 2000; Si et al., 2003; Prell et al., 2006), and does not mimic LPS or induce TNF- $\alpha$ (Manthey et al., 1993; Fitzpatrick et al., 2003). However, we found that docetaxel can reduce TNF- $\alpha$ production, which suggests it may have considerable use as an anti-inflammatory agent as well as a chemotherapeutic agent. The reduction in TNF- $\alpha$ could also be due to drug treatment affecting cytokine secretion and vesicle trafficking (Murray et al., 2005; Manderson et al., 2007). Additionally, we found latrunculin A decreased TNF- $\alpha$ production, which could be related to the fact that bacterial phagocytosis was impaired in the presence of latrunculin A. The levels of TNF- $\alpha$ produced in the presence of IFN- $\gamma$ and latrunculin A are similar to the levels produced during bacterial phagocytosis. This suggests that the presence of bacteria 
and LPS triggers TNF- $\alpha$ production, however, absence of phagocytosis leads to decreased TNF- $\alpha$ as killing mechanisms are not activated within the macrophage.

We found a significant decrease in TNF- $\alpha$ during bacterial killing in the presence of IFN- $\gamma$ and colchicine treatment. Colchicine is an anti-inflammatory microtubule depolymerizer and is known to decrease production of LPS-induced TNF- $\alpha$ (Rao et al., 1997; Isowa et al., 1999). Colchicine treatment also causes a downregulation of TNF- $\alpha \mathrm{R}$ (Ding et al., 1990a), further contributing to its anti-inflammatory effects. Additionally, we found colchicine treatment caused a significant decrease in $\mathrm{TNF}-\alpha$ production during bacterial killing in the absence of IFN- $\gamma$. This suggests that irrespective of macrophage activation, colchicine still exerts anti-inflammatory effects. Interestingly, in the presence of IFN- $\gamma$ during bacterial phagocytosis (Fig 7.4.2), colchicine significantly decreased TNF- $\alpha$ production. In contrast, in the absence of IFN- $\gamma$ stimulation during phagocytosis when TNF- $\alpha$ levels are low, there is no inhibition in the presence of colchicine. Overall this data suggests that colchicine is an effective anti-inflammatory drug and its effects on TNF- $\alpha$ and pro-inflammatory mediators are independent of the presence of LPS or macrophage activation status. It also demonstrates that the anti-inflammatory properties are dependent only on the levels of inflammatory cytokine irrespective of the processes such as phagocytosis or bacterial killing.

Overall during bacterial phagocytosis, there were no significant effects seen with activating macrophages as compared to lack of activation. However during bacterial killing, activation of macrophages led to significant increases in TNF- $\alpha$ production. The increase in TNF- $\alpha$ was modulated in the presence of high dose docetaxel, latrunculin A and colchicine, 
however, treatment with paclitaxel or peloruside A did not have any affect on the high levels of TNF- $\alpha$, which was surprising in the case of peloruside A. We also found colchicine treatment reduced TNF- $\alpha$ independent of macrophage activation status and phagocytic or bactericidal activities. Taken together the data suggests that inflammatory cytokine production during bacterial phagocytosis and killing by MSD treated macrophages is not altered. 


\section{Chapter 8: General Discussion}

\subsection{Review of overall findings}

This thesis focused on the effects exerted by MSD on macrophage function. In particular, it investigated the effects of MSD on endocytic and killing processes within the macrophage. We used the RAW264.7 and J774.2 murine macrophage cell lines to study these processes. While MSD are cytotoxic to dividing cells (Zhou et al., 2005; Schmidt et $a l ., 2007)$, over the period of our experiments, the cells were not dividing and we could examine effects distinct from mitosis related cytotoxic effects.

This study focused on two main endocytic pathways; receptor mediated endocytosis and phagocytosis. The rationale of this study was based on the fact that there are few studies that investigate the effects of MSD on non-proliferating cells. However, immune cells such as the macrophage depend on functional microtubules for vesicle trafficking, phagoslysosomal fusion, antigen processing, and cytokine delivery (Ostlund et al., 1979; Peachman et al., 2004; Murray et al., 2005; Manderson et al., 2007). We were interested in studying the effects that MSD dependent microtubule stabilization would have on receptor mediated endocytosis, as well as bacterial phagocytosis and killing. Additionally, some of the MSD have immunomodulatory effects or exhibit LPS mimicry (Ding et al., 1990b; Herbst et al., 2003; Si et al., 2003; Crume et al., 2007). MSD can also affect cytokine production independently to causing microtubule stabilization (Tsavaris et al., 2002; Crume et al., 2007). This can directly alter processes dependent on cytokine production such as bacterial killing (Havell, 1989) We also studied the effects of MSD during optimal macrophage activation in the presence of IFN- $\gamma$ and the effect of MSD when this activation was lacking. 
Our investigations also expanded upon previous work in our lab by showing that MSD did not inhibit production of TNF- $\alpha$ by RAW264.7 murine macrophages in the presence of limiting LPS. This was in contrast to findings that paclitaxel reduced levels of TNF- $\alpha$ in the presence of LPS below $20 \mathrm{ng} / \mathrm{ml}$ (Crume et al., 2007; Robinson, 2009). The RAW264.7 cells also did not exhibit increased metabolic rates in the presence of paclitaxel, in contrast to BMDM which did increase their metabolic rates when treated with paclitaxel (Crume et al., 2007; Robinson, 2009). RAW264.7 cells were extremely sensitive to LPS stimulation and treatment of cells with LPS alone at doses as low as $0.3 \mathrm{ng} / \mathrm{ml}$ resulted in high levels of TNF- $\alpha$ being produced.

Our work involving LDL and ac-LDL endocytosis demonstrated that paclitaxel treatment did not impair receptor mediated endocytic processes, even at high doses over 4 hours of treatment. However during these assays we looked at early uptake and endocytic processes and found no impairment. This is supported by studies where colchicine, which is a microtubule destabilizer, did not affect binding of LDL to cell receptors and endocytic rates were lowered, but LDL still accumulated in the cells (Ostlund et al., 1979). However, studies have shown that colchicine treatment inhibited degradation of these particles, as transport from the early endosome to the late endosome or lysozome was inhibited (Ostlund et al., 1979; Gruenberg et al., 1989). These findings support a role for microtubules in transport of vesicles and the studies suggest that while initial uptake is not impaired, processes such as lysozomal degradation are impaired. It is possible that treatment with paclitaxel or other MSDs would impair degradation of endocytosed particles while maintaining the initial uptake of these particles. Our studies also suggest that receptor 
function is not affected by paclitaxel, and the endocytic rate is maintained, which suggests receptor recycling is unaffected.

Our work showed for the first time that phagocytosis and killing of live bacteria in the presence of paclitaxel, peloruside A or docetaxel was not impaired. Understanding effects of MSD on microtubule-dependent cellular processes other than cell division is important as investigations into compounds that function as chemotherapeutics and immunomodulators increase (Chan et al., 2000; Garnett et al., 2008; Zitvogel et al., 2008; Miller et al., 2010). We found that in the absence of optimal activation with IFN- $\gamma$, MSD maintained phagocytic activity and the data suggests that these compounds stimulated macrophages to respond to bacterial stimulus. Additionally we investigated the effects of these compounds on inflammatory cytokine production. We found that there were no alterations to TNF- $\alpha$ production during phagocytosis or killing, however NO production was significantly lowered in the presence of high doses of MSD during bacterial phagocytosis, suggesting that although macrophages maintain response to bacterial presence, the initial cytokine response to the presence of LPS may be altered due to microtubule stabilization.

Additionally to investigating paclitaxel, docetaxel and peloruside A, we also studied the effects of 3 novel compounds zampanolide, ixabepilone and mycothiazole. Our study is the first to investigate the effects of these 3 compounds on macrophage response to live bacteria. Perhaps the most interesting effects regarding macrophage responses to bacteria were seen in the presence of zampanolide. Our data provides evidence that treatment with the MSD zampanolide significantly impairs bacterial phagocytosis and killing. We also 
found that treatment with zampanolide significantly affects TNF- $\alpha$ and NO production. It remains to be seen if the effects on macrophage function are a result of impaired cytokine production or an unknown intracellular target of zampanolide, since data from other MSD tested suggests that microtubule stabilization does not impair bacterial phagocytosis or killing.

We also found that ixabepilone, which is currently used clinically as a chemotherapeutic, enhances bacterial killing. However this MSD does not alter phagocytic efficiency or cytokine production. In contrast, mycothiazole impairs bacterial killing, however it does not alter cytokine production or phagocytic ability. The mode of action of mycothiazole is as yet unknown (Miller et al., 2010) and it will be extremely interesting to investigate this in light of current findings.

Overall our findings suggest that initial endocytic processes within the macrophage, although dependent on microtubules, are not immediately affected by the presence of MSD. It is possible that treatment with MSD will alter or inhibit later aspects of endocytosis, such as vesicle and antigen trafficking. When interpreting these results we should remember that these results are based in an in vitro situation and in vivo there are multiple factors that could alter the interaction of MSD with macrophages, thus resulting in different effects or outcomes.

Improved understanding of how MSD can affect immune responses is vital with the current expansion of this field. We have seen how these cytotoxic anticancer drugs have additional effects on the immune system, and it is only by understanding these interactions that the true potential of these compounds can be harnessed for their use as effective 
therapeutics. While nature has provided excellent potential chemotherapeutics in the form of MTA, an in-depth understanding of their side effects on immune cells are needed before these compounds are used, not only as chemotherapeutics but also as immune modifiers. We have seen the potential of MSD to be used as immune modifiers in autoimmune diseases (Cao et al., 2000; Crume et al., 2009). We hope that with in-depth understanding of the immunopharmacology and toxic effects of of these compounds at high doses (Rowinsky et al., 1995; Panchagnula, 1998), we will be better equipped to use these compounds with improved efficiency, lower toxicity and as chemoimmunotherapeutics.

\subsection{Future Directions}

This study demonstrates that MSD treatment can affect immune cell function and cytokine production. It opens new avenues for extending the findings reported in this study, particularly in regards to studying effects of MSD treatment on BMDM during bacterial phagocytosis and killing. Future studies would likely involve studying the effects of MSD on antigen trafficking and presentation, as this would help elucidate the effects of MSD on the role of the ER and golgi in trafficking and antigen presentation on MHC complexes. It is possible that activation of the adaptive immune system would be impaired in the presence of MSD and this would be a beneficial study.

Further pathways open for investigation are understanding the effects of MSD treatment, particularly paclitaxel, on the kinetics of bacterial phagocytosis. The confocal microscope techniques used in this research offer a good starting point to investigate the uptake kinetics of pHrodo. Additionally the role of the lysosome and lysosomal fusion during bacterial killing would also be interesting to investigate, as MSD treatment could alter the efficiency with which this process occurs. 
Numerous questions still remain unanswered, in particular regarding the effects of zampanolide on macrophage endocytic processes and cytokine production. Zampanolide has recently been shown to be an MSD; however its effects in this study are dramatically in contrast with all other MSD studied. It would be extremely interesting to investigate possible reasons for the effects seen with zampanolide, as the data from this study suggests that zampanolide could have alternative cellular targets than just microtubules. The cytotoxic effects of zampanolide on RAW264.7 cells would also be a good point to investigate in light of the effects of this compound on the RAW264.7 cell line.

Another interesting avenue to investigate would be the effects seen with peloruside A in RAW264.7 macrophages, which were in contrast to effects seen with BMDM. While we found that RAW264.7 cells are extremely sensitive to LPS stimulation, even at low doses, this does not completely explain the lack of downregulation to TNF- $\alpha$ and NO production in peloruside A treated macrophages. It would also be of great interest to study bacterial phagocytosis and killing in peloruside treated BMDM.

Lastly, a valuable avenue to follow would be investigating macrophage bactericidal activity in vivo in the presence of MSD treatment. This would allow studying MSD effects on macrophages as well as other immune cells, in the presence of MSD treatment, when multiple pathways are able to interact with each other. This would provide a good understanding of the dosing regime required and the possible toxic side effects, while also providing a picture of the efficiency of immune responses to bacterial stimulation in vivo in the presence of MSD. 


\subsection{Final conclusions}

This study set out to investigate the immune-mediated effects of MSDs on macrophages. In particular we studied the ability of MSD treated macrophages to phagocytose and kill bacteria. We also investigated the endocytosis of ligands via cell surface receptors in these MSD treated macrophages. Another aspect studied in this project was the effects of MSD on cytokine production. Firstly, we found that treatment with certain MSDs did not inhibit bacterial phagocytosis or killing. However other MSDs did inhibit macrophage function suggesting that different MSDs can alter immune function. Furthermore, cytokine production was also inhibited to varying degrees. Taken together, the data suggests that overall, MSD directly affect cytokine production causing indirect effects to macrophage function. Secondly, we found that zampanolide is far more potent and inhibits macrophage function significantly, suggesting that for clinical development the side effects of this compound need to be studied extensively. This project is the first to explore the effects of MSD on macrophage phagocytosis of live E.coli bacteria. The results suggest that during clinical use of MSD, the ability of patients to fight off bacterial infections maybe altered due to impaired cytokine production and altered kinetics during macrophage responses to the presence of bacteria. 


\section{CHAPTER 9: REFERENCES}

Aderem, A. \& Underhill, D.M. (1999). Mechanisms of Phagocytosis in Macrophages. Annual Review of Immunology 17, 593-623.

Aggarwal, B.B. (2003). Signalling pathways of the TNF superfamily: a Double-Edged Sword. Nature Reviews - Immunology 3, 745-756.

Al-Haddad, A., Shonn, M.A., Redlich, B., Blocker, A., Burkhardt, J.K., Yu, H., Hammer, J.A., III, Weiss, D.G., Steffen, W., Griffiths, G. \& Kuznetsov, S.A. (2001). Myosin Va Bound to Phagosomes Binds to F-Actin and Delays Microtubule-dependent Motility. Molecular Biology of the Cell 12, 2742-2755.

Al-Mariri, A. (2008). Survival and Replication of Escherichia Coli O157:H7 Inside the Mice Peritoneal Macrophages. Brazilian Journal of Microbiology 39, 5-9.

Allen, J.N., Liao, Z., Moore, S.A. \& Wewers, M.D. (1997a). Changes in Mononuclear Phagocyte Microtubules After Endotoxin Stimulation. II. Changes in Microtubule Composition. American Journal of Respiratory Cell and Molecular Biology 16, 127-132.

Allen, J.N., Moore, S.A., Liao, Z. \& Wewers, M.D. (1997b). Changes in Mononuclear Phagocyte Microtubules After Endotoxin Stimulation. I. Changes in Microtubule Stability. American Journal of Respiratory Cell and Molecular Biology 16, 119-126.

Allen, L. \& Aderem, A. (1996). Molecular Definition of Distinct Cytoskeletal Structures Involved in Complement- and Fc Receptor-mediated Phagocytosis in Macrophages. The Journal of Experimental Medicine 184, 627-637.

Altmann, K.-H. (2001). Microtubule-stabilizing Agents: a Growing Class of Important Anti-cancer Drugs. Current Opinion in Chemical Biology, 424 - 431.

Altmann, K.-H. (2003). Epothilone B and its Analogs - A New Family of Anticancer Agents. Mini Reviews in Medicinal Chemistry 3, 149.

American Type Culture Collection. (2007) Passage Number Effects in Cell Llines. Why they happen and what you can do about it. In: ATCC Technical Bulletin No 7. American Type Culture Collection (ATCC).

Anand, R.J., Gribar, S.C., Li, J., Kohler, J.W., Branca, M.F., Dubowski, T., Sodhi, C.P. \& Hackam, D.J. (2007). Hypoxia Causes An Increase in Phagocytosis by Macrophages in a HIF-1a-dependent manner. Journal of Leukocyte Biology 82, 1257-1265.

Anderson, R., G., Brown, M., S., Beisiegel, U. \& Goldstein, J., L. (1982). Surface Distribution and Recycling of the Low Density Lipoprotein Receptor as Visualized with Antireceptor Antibodies. The Journal of Cell Biology 93, 523-531. 
Anderson, R.G.W., Brown, M.S. \& Goldstein, J.L. (1977). Role of the Coated Endocytic Vesicle in the Uptake of Receptor-bound Low Density Lipoprotein in Human Fibroblasts. Cell 10, 351-364.

Apodaca, G. (2001). Endocytic Traffic in Polarized Epithelial Cells: Role of the Actin and Microtubule Cytoskeleton. Traffic 2, 149-159.

Archer, S. (1993). Measurement of nitric oxide in biological models. The FASEB Journal 7, 349360 .

Areschoug, T., Waldemarsson, J. \& Gordon, S. (2008). Evasion of macrophage scavenger receptor A-mediated recognition by pathogenic streptococci. European Journal of Immunology 38, 30683079.

Bach, E.A., Aguet, M. \& Schreiber, R.D. (1997). The IFN- $\gamma$ Receptor: A Paradigm for Cytokine Receptor Signaling. Annual Review of Immunology 15, 563-591.

Baeuerle, P.A. \& Henkel, T. (1994). Function and Activation of NF-kB in the Immune System. Annual Review of Immunology 12, 141-179.

Berghaus, L.J., Moore, J.N., Hurley, D.J., Vandenplas, M.L., Fortes, B.P., Wolfert, M.A. \& Boons, G.-J. (2009). Innate immune responses of primary murine macrophage-lineage cells and RAW 264.7 cells to ligands of Toll-like receptors 2, 3, and 4. Comparative Immunology, Microbiology and Infectious Diseases doi:10.1016/j.cimid.2009.07.001.

Berridge, M.V., Tan, A.S., McCoy, K.D. \& Wang, R. (1996). The Biochemical and Cellular Basis of Cell Proliferation Assays that use Tetrazolium Salts. Biochemica 4, 15-20.

Bhattacharyya, B., Panda, D., Gupta, S. \& Banerjee, M. (2008). Anti-mitotic Activity of Colchicine and the Structural Basis for its Interaction with Tubulin. Medicinal Research Reviews 28, 155-183.

Bingle, L., Brown, N.J. \& Lewis, C.E. (2002). The role of tumor-associated macrophages in tumor progression: implications for new anticancer therapies. Journal of Pathology 196, 254-256.

Binker, M.G., Zhao, D.Y., Pang, S.J.Y. \& Harrison, R.E. (2007). Cytoplasmic Linker Protein-170 Enhances Spreading and Phagocytosis in Activated Macrophages by Stabilizing Microtubules. The Journal of Immunology 179, 3780-3791.

Blocker, A., Griffiths, G., Olivo, J.-C., Hyman, A.A. \& Severin, F.F. (1998). A Role for Microtubule Dynamics in Phagosome Movement. The Journal of Cell Science 111, 303-312.

Blocker, A., Severin, F.F., Burkhardt, J.K., Bingham, J.B., Yu, H., Olivo, J.-C., Schroer, T.A., Hyman, A.A. \& Griffiths, G. (1997). Molecular Requirements for Bi-Directional Movement of Phagosomes Along Microtubules. The Journal of Cell Biology 137, 113-129. 
Blocker, A., Severin, F.F., Habermann, A., Hyman, A.A., Griffiths, G. \& Burkhardt, J.K. (1996). Microtubule-Associated Protein-dependent Binding of Phagosomes to Microtubules. The Journal of Biological Chemistry 271, 3803-3811.

Boehm, U., Klamp, T., Groot, M. \& Howard, J.C. (1997). Cellular Responses to Interferon- $\beta$. Annual Review of Immunology 15, 749-795.

Bogdan, C. \& Ding, A. (1992). Taxol, a Microtubule-stabilizing Antineoplastic Agent, Induces Expression of Tumor Necrosis Factor- $\alpha$ and Interleukin-1 in Macrophages. Journal of Leukocyte Biology 52, 119-121.

Bogdan, C., Röllinghoff, M. \& Diefenbach, A. (2000). The Role of Nitric Oxide in Innate Immunity. Immunological Reviews 173, 17-26.

Bollag, D.M., McQueney, P.A., Zhu, J., Hensens, O., Koupal, L., Liesch, J., Goetz, M., Lazarides, E. \& Woods, C.M. (1995). Epothilones, a New Class of Microtubule-stabilizing Agents with a Taxol-like Mechanism of Action. Cancer Research 55, 2325-2333.

Brahn, E., Tang, C. \& Banquerigo, M.L. (1994). Regression of Collagen-Induced Arthritis with Taxol, a Microtubule Stabilizer. Arthritis and Rheumatism 37, 839-845.

Brown, M., Goldstein, J., Krieger, M., Ho, Y. \& Anderson, R. (1979a). Reversible Accumulation of Cholesteryl Esters in Macrophages Incubated with Acetylated Lipoproteins. The Journal of Cell Biology 82, 597-613.

Brown, M.S. \& Goldstein, J.L. (1979b). Receptor-mediated Endocytosis: Insights from the Lipoprotein Receptor System. Proceedings of the National Academy of Sciences of the United States of America 76, 3330-3337.

Brown, M.S. \& Goldstein, J.L. (1983). Lipoprotein Metabolism in the Macrophage: Implications for Cholesterol Deposition in Atherosclerosis. Annual Review of Biochemistry 52, 223-261.

Bruckdorfer, R. (2005). The Basics about Nitric Oxide. Molecular Aspects of Medicine 26, 3-31.

Burkhart, C.A., Berman, J.W., Swindell, C.S. \& Horwitz, S.B. (1994). Relationship between the Structure of Taxol and Other Taxanes on Induction of Tumor Necrosis Factor- $\alpha$ Gene Expression and Cytotoxicity. Cancer Research 54, 5779-5782.

Burkhart, C.A., Kavallaris, M. \& Horwitz, S.B. (2001). The Role of $\beta$-tubulin Isotypes in Resistance to Antimitotic Drugs. Biochimica et Biophysica Acta (BBA) - Reviews on Cancer 1471, O1-O9.

Byrd-Leifer C.A., Ellen F Block, Kiyoshi Takeda, Shizuo Akira \& Ding., A. (2001). The Role of MyD88 and TLR-4 in the LPS-mimetic Activity of Taxol. European Journal of Immunology 31, 2448-2457. 
Campbell, P.A., Canono, B.P. \& Drevets, D.A. (1994). Macrophages and Monocytes : Measurement of Bacterial Ingestion and Killing by Macrophages. Current Protocols in Immunology Supplement 12, 14.6.1-14.6.13.

Cao, L., Sun, D., Cruz, T., Moscarello, M.A., Ludwin, S.K. \& Whitaker, J.N. (2000). Inhibition of Experimental Allergic Encephalomyelitis in the Lewis rat by Paclitaxel. Journal of Neuroimmunology 108, 103-111.

Cao, W.M., Murao, K., Imachi, H., Sato, M., Nakano, T., Kodama, T., Sasaguri, Y., Wong, N.C.W., Takahara, J. \& Ishida, T. (2001). Phosphatidylinositol 3-OH Kinase-Akt/Protein Kinase B Pathway Mediates Gas6 Induction of Scavenger Receptor A in Immortalized Human Vascular Smooth Muscle Cell Line. Arteriosclerosis, Thrombosis, and Vascular Biology 21, 1592-1597.

Carswell, E.A., Old, L.J., Kassel, R.L., Green, S., Fiore, N. \& Williamson, B. (1975). An Endotoxin-induced Serum Factor that causes Necrosis of Tumors. Proceedings of the National Academy of Sciences of the United States of America 72, 3666-3670.

Cassimeris, L.U., Walker, R.A., Pryer, N.K. \& Salmon, E.D. (1987). Dynamic Instability of Microtubules. Bioessays 7, 149 - 154.

Chamulitrat, W., Blazka, M.E., Jordan, S.J., Luster, M.I. \& Mason, R.P. (1995). Tumor necrosis factor-[alpha] and nitric oxide production in endotoxin-primed rats administered carbon tetrachloride. Life Sciences 57, 2273-2280.

Chan, O.T.M. \& Yang, L.-X. (2000). The Immunological Effects of Taxanes. Cancer Immunology, Immunotherapy 49, 181-185.

Conde, C. \& Cáceres, A. (2009). Microtubule Assembly, Organization and Dynamics in Axons and Dendrites. Nature Reviews - Neuroscience 10, 319 - 332.

Conlin, A., Fornier, M., Hudis, C., Kar, S. \& Kirkpatrick, P. (2007). Ixabepilone. Nature Reviews Drug Discovery 6, 953-954.

Correia, J.J. \& Lobert, S. (2008) Molecular Mechanism of Microtubule Acting Cancer Drugs. In: T. Fojo (Ed) Cancer Drug Discovery and Development: The Role of Microtubules in Cell Biology, Neurobiology and Oncology. Humana Press, Totowa, NJ, p. 21-46.

Coue, M., Brenner, S.L., Spector, I. \& Korn, E.D. (1987). Inhibition of actin polymerization by Latrunculin A. Federation of European Biochemical Societies 213, 316-318.

Crume, K.P., Miller, J.H. \& LaFlamme, A.C. (2007). Peloruside A, an Antimitotic Agent, Specifically Decreases Tumor Necrosis Factor-a Production by Lipopolysaccharide-Stimulated Murine Macrophages. Experimental Biology and Medicine 232, 607-613.

Crume, K.P., O'Sullivan, D., Miller, J.H., Northcote, P.T. \& La Flamme, A.C. (2009). Delaying the Onset of Experimental Autoimmune Encephalomyelitis with the Microtubule-stabilizing Compounds, Paclitaxel and Peloruside A. Journal of Leukocyte Biology, jlb.0908541. 
Dempsey, P.W., Doyle, S.E., He, J.Q. \& Cheng, G. (2003). The Signaling Adaptors and Pathways Activated by TNF Superfamily. Cytokine \& Growth Factor Reviews 14, 193-209.

Desai, A. \& Mitchson, T.J. (1997). Microtubule Polymerization Dynamics. Annual Reviews Cell Development Biology 13, 83 - 117.

Desjardins, M., Celis, J.E., van Meer, G., Dieplinger, H., Jahraus, A., Griffiths, G. \& Huber, L.A. (1994a). Molecular Characterization of Phagosomes. The Journal of Biological Chemistry 269, 32194-32200.

Desjardins, M., Huber, L., Parton, R. \& Griffiths, G. (1994b). Biogenesis of Phagolysosomes proceeds through a Sequential Series of Interactions with the Endocytic Apparatus. The Journal of Cell Biology 124, 677-688.

Dialynas, D.P., Tan, P.C., Huhn, G.D. \& Yu, J. (1997). Characterization of a New Human Macrophage Cell Line 2MAC. Cellular Immunology 177, 182-193.

Dinarello, C.A. (2007). Historical Insights into Cytokines. European Journal of Immunology 37, S34-S45.

Ding, A., Nathan, C. \& Stuehr, D. (1988). Release of Reactive Nitrogen Intermediates and Reactive Oxygen Intermediates from Mouse Peritoneal Macrophages. Comparison of Activating Cytokines and Evidence for Independent Production. The Journal of Immunology 141, 2407-2412.

Ding, A., Sanchez, E. \& Nathan, C. (1993). Taxol shares the Ability of Bacterial Lipopolysaccharide to induce Tyrosine Phosphorylation of Microtubule-associated Protein Kinase. The Journal of Immunology 151, 5596-5602.

Ding, A., Sanchez, E., Tancinco, M. \& Nathan, C. (1992). Interactions of Bacterial Lipopolysaccharide with Microtubule Proteins. The Journal of Immunology 148, 2853-2858.

Ding, A.H., Porteu, F., Sanchez, E. \& Nathan, C.F. (1990a). Downregulation of Tumor Necrosis Factor Receptors on Macrophages and Endothelial cells by Microtubule Depolymerizing Agents. The Journal of Experimental Medicine 171, 715-727.

Ding, A.H., Porteu, F., Sanchez, E. \& Nathan, C.F. (1990b). Shared Actions of Endotoxin and Taxol on TNF Receptors and TNF Release. Science 248, 370-372.

Ding, A.H., Sanchez, E., Srimal, S. \& Nathan, C.F. (1989). Macrophages Rapidly Internalize their Tumor Necrosis Factor Receptors in Response to Bacterial Lipopolysaccharide. Journal of Biological Chemistry 264, 3924-3929.

Dobrovolskaia, M.A. \& Vogel, S.N. (2002). Toll receptors, CD14, and macrophage activation and deactivation by LPS. Microbes and Infection 4, 903-914. 
Donovan, D. \& Vahdat, L.T. (2008). Epothilones: Clinical Update and Future Directions. (Special Issue on Targeted Therapy). Oncology 22, 408.

Downing, K.H. \& Nogales, E. (1998a). Tubulin and Microtubule Structure. Current Opinion in Cell Biology 10, 16 - 22.

Downing, K.H. \& Nogales, E. (1998b). Tubulin Structure: Insights into Microtubule Properties and Functions. Current Opinion in Structural Biology 8, 785 - 791.

Ehlenberger, A. \& Nussenzweig, V. (1977). The Role of Membrane Receptors for C3b and C3d in Phagocytosis. The Journal of Experimental Medicine 145, 357-371.

Elsinghorst, E.A. (1994). Measurement of Invasion by Gentamicin Resistance. Methods in Enzymology 236, 405-420.

Feng, Y.H., Zou, J.P. \& Li, X.Y. (2002). Effects of resveratrol and ethanol on production of proinflammatory factors from endotoxin activated murine macrophages. Acta Pharmacologica Sinica $23,1002-1006$.

Field, J.J., Singh, A.J., Kanakkanthara, A., Halafihi, T.i., Northcote, P.T. \& Miller, J.H. (2009). Microtubule-Stabilizing Activity of Zampanolide, a Potent Macrolide Isolated from the Tongan Marine Sponge Cacospongia mycofijiensis. Journal of Medicinal Chemistry 52, 7328-7332.

Fitzgerald, M.L., Moore, K.J., Freeman, M.W. \& Reed, G.L. (2000). Lipopolysaccharide Induces Scavenger Receptor A Expression in Mouse Macrophages: A Divergent Response Relative to Human THP-1 Monocyte/Macrophages. The Journal of Immunology 164, 2692-2700.

Fitzpatrick, F.A. \& Wheeler, R. (2003). The immunopharmacology of paclitaxel (Taxol®), docetaxel (Taxotere $\left.{ }^{\circledR}\right)$, and related agents. International Immunopharmacology 3, 1699-1714.

Fong, Y., Tracey, K.J., Moldawer, L.L., Hesse, D.G., Manogue, K.B., Kenney, J.S., Lee, A.T., Kuo, G.C., Allison, A.C., Lowry, S.F. \& Cerami, A. (1989). Antibodies to Cachectin/Tumor Necrosis Factor Reduce Interleukin $1 \beta$ and Interleukin 6 Appearance during Lethal Bacteremia. Journal of Experimental Medicine 170, 1627-1633.

Forstermann, U., Closs, E., Pollock, J., Nakane, M., Schwarz, P., Gath, I. \& Kleinert, H. (1994). Nitric Oxide Synthase Isozymes. Characterization, Purification, Molecular Cloning, and Functions. Hypertension 23, 1121-1131.

Fujihara, M., Muroi, M., Tanamoto, K.-i., Suzuki, T., Azuma, H. \& Ikeda, H. (2003). Molecular Mechanisms of Macrophage Activation and Deactivation by Lipopolysaccharide: Roles of the Receptor Complex. Pharmacology \& Therapeutics 100, 171-194.

Gaffney, J., West, D., Arnold, F., Sattar, A. \& Kumar, S. (1985). Differences in the Uptake of Modified Low Density Lipoproteins by Tissue Cultured Endothelial Cells. Journal of Cell Science 79, 317-325. 
Gaitanos, T.N., Buey, R.M., Diaz, J.F., Northcote, P.T., Teesdale-Spittle, P., Andreu, J.M. \& Miller, J.H. (2004). Peloruside A Does Not Bind to the Taxoid Site on $\beta$-Tubulin and Retains Its Activity in Multidrug-Resistant Cell Lines. Cancer Research 64, 5063-5067.

Gallagher, R., Collins, S., Trujillo, J., McCredie, K., Ahearn, M., Tsai, S., Metzgar, R., Aulakh, G., Ting, R., Ruscetti, F. \& Gallo, R. (1979). Characterization of the Continuous, Differentiating Myeloid Cell line (HL-60) from a Patient with Acute Promyelocytic Leukemia. Blood 54, 713-733.

Garnett, C.T., Schlom, J. \& Hodge, J.W. (2008). Combination of Docetaxel and Recombinant Vaccine Enhances T-cell Responses and Antitumor Activity: Effects of Docetaxel on Immune Enhancement. Clinical Cancer Research 14, 3536-3544.

Gelderblom, H., Verweij, J., Nooter, K. \& Sparreboom, A. (2001). Cremophor EL: the Drawbacks and Advantages of Vehicle Selection for Drug Formulation. European Journal of Cancer 37, 15901598 .

Gelfand, V.I. \& Bershadsky, A.D. (1991). Microtubule Dynamics: Mechanism, Regulation and Function. Annual Review of Cell Biology 7, 93 - 116.

Geller, D.A., Nussler, A.K., Di Silvio, M., Lowenstein, C.J., Shapiro, R.A., Wang, S.C., Simmons, R.L. \& Billiar, T.R. (1993). Cytokines, Endotoxin, and Glucocorticoids Regulate the Expression of Inducible Nitric Oxide Synthase in Hepatocytes. Proceedings of the National Academy of Sciences of the United States of America 90, 522-526.

Geney, R., Ungureanu, I.M., Li, D. \& Ojima, I. (2002). Overcoming Multidrug Resistance in Taxane Chemotherapy. Clinical Chemistry and Laboratory Medicine 40, 918-925.

Giannakakou, P., Gussio, R., Nogales, E., Downing, K.H., Zaharevitz, D., Bollbuck, B., Poy, G., Sackett, D., Nicolaou, K.C. \& Fojo, T. (2000). A Common Pharmacophore for Epothilone and Taxanes: Molecular basis for Drug Resistance conferred by Tubulin Mutations in Human Cancer Cells. Proceedings of the National Academy of Sciences of the United States of America 97, 29042909.

Goldstein, J.L., Ho, Y.K., Basu, S.K. \& Brown, M.S. (1979). Binding Site on Macrophages that Mediates Uptake and Degradation of Acetylated Low Density Lipoprotein, Producing Massive Cholesterol Deposition. Proceedings of the National Academy of Sciences of the United States of America 76, 333-337.

Goltz, J.S., Wolkoff, A.W., Novikoff, P.M., Stockert, R.J. \& Satir, P. (1992). A Role for Microtubules in Sorting Endocytic Vesicles in Rat Hepatocytes. Proceedings of the National Academy of Sciences of the United States of America 89, 7026-7030.

Goncalves, A., Braguer, D., Kamath, K., Martello, L., Briand, C., Horwitz, S., Wilson, L. \& Jordan, M.A. (2001). Resistance to Taxol in Lung Cancer Cells associated with Increased Microtubule Dynamics. Proceedings of the National Academy of Sciences of the United States of America 98, 11737-11742. 
Goodin, S. (2008). Ixabepilone: A novel Microtubule-Stabilizing Agent for the Treatment of Metastatic Breast Cancer. American Journal of Health-System Pharmacy 65, 2017-2026.

Goodin, S., Kane, M.P. \& Rubin, E.H. (2004). Epothilones: Mechanism of Action and Biologic Activity. Journal of Clinical Oncology 22, 2015-2025.

Greenberg, S. \& Grinstein, S. (2002). Phagocytosis and Innate Immunity. Current Opinion in Immunology 14, 136-145.

Gruenberg, J., Griffiths, G. \& Howell, K.E. (1989). Characterization of the Early Endosome and Putative Endocytic Carrier Vesicles In-Vivo and with an Assay of Vesicle Fusion In-Vitro. The Journal of Cell Biology 108, 1301-1316.

Gupta, S. (2003). P-glycoprotein Expression in the Cells of the Immune System During Aging. Clinical and Applied Immunology Reviews 4, 59-70.

Haber, M., Burkhart, C.A., Regl, D.L., Madafiglio, J., Norris, M.D. \& Horwitz, S.B. (1995). Altered Expression of M2, the Class II -Tubulin Isotype, in a Murine J774.2 Cell Line with a High Level of Taxol Resistance. Journal of Biological Chemistry 270, 31269-31275.

Hamza, T., Barnett, J.B. \& Li, B. (2010). Interleukin 12 a Key Immunoregulatory Cytokine in Infection Applications. International Journal of Molecular Sciences 11, 789-806.

Harrison, R.E. \& Grinstein, S. (2002). Phagocytosis and the Microtubule Cytoskeleton. Biochemisty and Cell Biology 80, 509-515.

Havell, E.A. (1989). Evidence that Tumor Necrosis Factor has an Important Role in Antibacterial Resistance. Journal of Immunology 143, 2894-2899.

Henriksen, T., Mahoney, E.M. \& Steinberg, D. (1981). Enhanced Macrophage Degradation of Low Density Lipoprotein previously incubated with Cultured Endothelial Cells: Recognition by Receptors for Acetylated Low Density Lipoproteins. Proceedings of the National Academy of Sciences of the United States of America 78, 6499-6503.

Herbst, R.S. \& Khuri, F., R. (2003). Mode of Action of Docetaxel - A Basis for Combination with Novel Anti-Cancer Agents. Cancer Treatment Reviews 29.

Hermans, I.F., Silk, J.D., Yang, J., Palmowski, M.J., Gileadi, U., McCarthy, C., Salio, M., Ronchese, F. \& Cerundolo, V. (2004). The VITAL assay: a versatile fluorometric technique for assessing CTL- and NKT-mediated cytotoxicity against multiple targets in vitro and in vivo. Journal of Immunological Methods 285, 25-40.

Holan, V., Krulova, M., Zajicova, A. \& Pindjakova, J. (2001). Nitric Oxide as a Regulatory and Effector Molecule in the Immune System. Molecular Immunology 38, 989-995. 
Hood, K.A., Backstrom, B.T., West, L.M., Northcote, P.T., Berridge, M.V. \& Miller, J.H. (2001). The novel cytotoxic sponge metabolite peloruside A, structurally similar to bryostatin-1, has unique bioactivity independent of protein kinase C. Anti-Cancer Drug Design 16, 155-166.

Hood, K.A., West, L.M., Rouwe, B., Northcote, P.T., Berridge, M.V., Wakefield, S.J. \& Miller, J.H. (2002). Peloruside A, a Novel Antimitotic Agent with Paclitaxel-like Microtubule-stabilizing Activity. Cancer Research 62, 3356-3360.

Howard, J. \& Hyman, A.A. (2003). Dynamics and mechanics of the microtubule plus end. Nature 422, 753-758.

Hsueh, R. \& Roach, T. (2008) Passage Procedure for RAW264.7 cells. In: AfCS Procedure Protocol PP00000159. American Type Culture Collection, p. 1-3.

Huzil, J.T., Chik, J.K., Slysz, G.W., Freedman, H., Tuszynski, J., Taylor, R.E., Sackett, D.L. \& Schriemer, D.C. (2008). A Unique Mode of Microtubule Stabilization Induced by Peloruside A. Journal of Molecular Biology 378, 1016-1030.

Isowa, N., Xavier, A.M., Dziak, E., Opas, M., McRitchie, D.I., Slutsky, A.S., Keshavjee, S.H. \& Liu, M. (1999). LPS-induced depolymerization of cytoskeleton and its role in TNF- production by rat pneumocytes. American Journal of Physiology - Lung, Cellular and Molecular Physiology 277, L606-L615.

Javeed, A., Ashraf, M., Riaz, A., Ghafoor, A., Afzal, S. \& Mukhtar, M.M. (2009). Paclitaxel and Immune System. European Journal of Pharmaceutical Sciences 38, 283-290.

Jimenez-Barbero, J., Canales, A., Northcote, P.T., Buey, R.M., Andreu, J.M. \& Diaz, J.F. (2006). NMR Determination of the Bioactive Conformation of Peloruside A Bound To Microtubules. Journal of the American Chemical Society 128, 8757-8765.

Jordan, M.A. \& Kamath, K. (2007). How Do Microtubule-Targeted Drugs Work? An Overview. Current Cancer Drug Targets 7, 730-742.

Jordan, M.A., Toso, R.J., Thrower, D. \& Wilson, L. (1993). Mechanism of Mitotic Block and Inhibition of Cell Proliferation by Taxol at Low Concentrations. Proceedings of the National Academy of Sciences of the United States of America 90, 9552-9556.

Jordan, M.A. \& Wilson, L. (2004). Microtubules as a Target for Anticancer Drugs. Nature Reviews Cancer 4, 253-265.

Jordan, M.A. \& Wilson, L. (2008) Microtubule Dynamics. In: M. Tito Fojo, PhD (Ed) The Role of Microtubules in Cell Biology, Neurobiology, and Oncology, p. 47-81.

Kaplan, G. (1977). Differences in the Mode of Phagocytosis with Fc and C3 Receptors in Macrophages. Scandinavian Journal of Immunology 6, 797-807. 
Karpuzoglu, E. \& Ahmed, A.S. (2006). Estrogen Regulation of Nitric Oxide and Inducible Nitric Oxide Synthase (iNOS) in Immnue Cells: Implications for Immunity, Autoimmune Diseases and Apoptosis. Nitric Oxide 15, 177-186.

Kasugai, S., Hasegawa, N. \& Ogura, H. (1990). A Simple In-Vitro Cytotoxicity Test Using the MTT (3-(4,5)-Dimethylthiazol-2-yl)-2,5-Diphenyl Tetrazolium Bromide) Colorimetric Assay: Analysis of Eugenol Toxicity on Dental Pulp Cells (RPC-C2A). Japan Journal of Pharmacology 52, 95-100.

Kavallaris, M. (2010). Microtubules and Resistance to Tubulin-Binding Agents. Nature Reviews Cancer 10, 194-204.

Kawasaki, K., Akashi, S., Shimazu, R., Yoshida, T., Miyake, K. \& Nishijima, M. (2000). Mouse Toll-like Receptor 4-MD-2 Complex Mediates Lipopolysaccharide-mimetic Signal Transduction by Taxol. Journal of Biological Chemistry 275, 2251-2254.

Kawasaki, K., Akashi, S., Shimazu, R., Yoshida, T., Miyake, K. \& Nishijima, M. (2001a). Involvement of TLR4/MD-2 Complex in Species-Specific Lipopolysaccharide-Mimetic Signal Transduction by Taxol. Journal of Endotoxin Research 7, 232-236.

Kawasaki, K., Gomi, K. \& Nishijima, M. (2001b). Cutting Edge: Gln22 of Mouse MD-2 Is Essential for Species-Specific Lipopolysaccharide Mimetic Action of Taxol. The Journal of Immunology 166, 11-14.

Kelling, J., Sullivan, K., Wilson, L. \& Jordan, M.A. (2003). Suppression of Centromere Dynamics by Taxol(R) in Living Osteosarcoma Cells. Cancer Research 63, 2794-2801.

Kelly, R.B. (1990). Microtubules, Membrane Traffic, and Cell Organization. Cell 61, 5-7.

Khandani, A., Eng, E., Jongstra-Bilen, J., Schreiber, A.D., Douda, D., Samavarchi-Tehrani, P. \& Harrison, R.E. (2007). Microtubules Regulate PI-3K Activity and Recruitment to the Phagocytic Cup during $\mathrm{Fc}-\gamma$ Receptor-mediated Phagocytosis in Non-Elicited Macrophages. Journal of Leukocyte Biology 82, 417-428.

Khazen, W., M'Bika, J.-P., Tomkiewicz, C., Benelli, C., Chany, C., Achour, A. \& Forest, C. (2005). Expression of Macrophage-Selective Markers in Human and Rodent Adipocytes. FEBS Letters 579, 5631-5634.

Kirikae, T., Ojima, I., Kirikae, F., Ma, Z., Kuduk, S.D., Slater, J.C., Takeuchi, C.S., Bounaud, P.-Y. \& Nakano, M. (1996). Structural Requirements of Taxoids for Nitric Oxide and Tumor Necrosis Factor Production by Murine Macrophages. Biochemical and Biophysical Research Communications 227, 227-235.

Klimp, A.H., de Vries, E.G.E., Scherphof, G.L. \& Daemen, T. (2002). A Potential Role of Macrophage Activation in the Treatment of Cancer. Critical Reviews in Oncology/Hematology 44, 143-161. 
Klippel, N. \& Bilitewski, U. (2007). Phagocytosis Assay Based on Living Candida Albicans for the Detection of Effects of Chemicals on Macrophage Function. Analytical Letters 40, 1400-1411.

Knowles, R.G. \& Moncada, S. (1994). Nitric Oxide Synthases in Mammals. Biochemical Journal 298, 249-0.

Koval, M., Preiter, K., Adles, C., Stahl, P.D. \& Steinberg, T.H. (1998). Size of IgG-Opsonized Particles Determines Macrophage Response during Internalization. Experimental Cell Research 242, 265-273.

Lambert, C., Desbarats, J., Arbour, N., Hall, J.A., Olivier, A., Bar-Or, A. \& Antel, J.P. (2008). Dendritic Cell Differentiation Signals Induce Anti-Inflammatory Properties in Human Adult Microglia. The Journal of Immunology 181, 8288-8297.

Laroux, F.S., Romero, X., Wetzler, L., Engel, P. \& Terhorst, C. (2005). MyD88 Controls Phagocyte NADPH Oxidase Function and Killing of Gram Negative Bacteria. The Journal of Immunology $175,5596-5600$.

Lechleider, R.J., Kaminskas, E., Jiang, X., Aziz, R., Bullock, J., Kasliwal, R., Harapanhalli, R., Pope, S., Sridhara, R., Leighton, J., Booth, B., Dagher, R., Justice, R. \& Pazdur, R. (2008). Ixabepilone in Combination with Capecitabine and as Monotherapy for Treatment of Advanced Breast Cancer Refractory to Previous Chemotherapies. Clinical Cancer Research 14, 4378-4384.

Lewkowicz, E., Herit, F., Le Clainche, C., Bourdoncle, P., Perez, F. \& Niedergang, F. (2008). The Microtubule-binding Protein CLIP-170 Coordinates mDial and Actin Reorganization during CR3Mediated Phagocytosis. The Journal of Cell Biology 183, 1287-1298.

Li, Q. \& Joshi, H.C. (1995). Gamma-Tubulin is a Minus End-specific Microtubule Binding Protein. Journal of Cell Biology 131, 207-214.

Lorsbach, R.B., Murphy, W.J., Lowenstein, C.J., Snyder, S.H. \& Russell, S.W. (1993). Expression of the Nitric Oxide Synthase Gene in Mouse Macrophages Activated for Tumor Cell Killing. Molecular Basis for the Synergy between Interferon- $\gamma$ and Lipopolysaccharide. Journal of Biological Chemistry 268, 1908-1913.

Luzio, J.P., Pryor, P.R. \& Bright, N.A. (2007). Lysosomes: Fusion and Function. Nature Reviews Molecular Cell Biology 8, 622-632.

Ma, X., Chow, J.M., Gri, G., Carra, G., Gerosa, F., Wolf, S.F., Dzialo, R. \& Trinchieri, G. (1996). The Interleukin 12-p40 Gene Promoter is Primed by Interferon- $\gamma$ in Monocytic Cells. The Journal of Experimental Medicine 183, 147-157.

Manderson, A.P., Kay, J.G., Hammond, L.A., Brown, D.L. \& Stow, J.L. (2007). Subcompartments of the Macrophage Recycling Endosome Direct the Differential Secretion of IL-6 and TNF- $\alpha$. Journal of Cell Biology 178, 57-69. 
Manthey, C.L., Brandes, M.E., Perera, P.Y. \& Vogel, S.N. (1992). Taxol Increases Steady-State Levels of Lipopolysaccharide Inducible Genes and Protein-Tyrosine Phosphorylation in Murine Macrophages. The Journal of Immunology 149, 2459-2465.

Manthey, C.L., Qureshi, N., Stutz, P.L. \& Vogel, S.N. (1993). Lipopolysaccharide Antagonists Block Taxol-induced Signaling in Murine Macrophages. Journal of Experimental Medicine 178, 695-702.

Maurya, M.R., Benner, C., Pradervand, S., Glass, C. \& Subramaniam, S. (2007) Systems Biology of Macrophages. In: Current Topics in Innate Immunity, p. 62-79.

Mellman, I. (1996). Endocytosis and Molecular Sorting Annual Review of Cell and Developmental Biology 12, 575-625.

Miksa, M., Komura, H., Wu, R., Shah, K.G. \& Wang, P. (2009). A Novel Method to Determine the Engulfment of Apoptotic Cells by Macrophages using pHrodo Succinimidyl Ester. Journal of Immunological Methods 342, 71-77.

Miliukiene, V.V., Biziuleviciene, G.J., Chaustova, L.P., Pilinkiene, A.V. \& Biziulevicius, G.A. (2007). Determination of Quantitative Parameters of Escherichia coli Phagocytosis by Mouse Peritoneal Macrophages. Cell and Tissue Biology 1, 446-450.

Miller, J.H., Singh, A.J. \& Northcote, P.T. (2010). Microtubule-Stabilizing Drugs from Marine Sponges: Focus on Peloruside A and Zampanolide. Marine Drugs 8, 1059-1079.

Mizuno, M. \& Singer, S.J. (1994). A possible role for stable microtubules in intracellular transport from the endoplasmic reticulum to the Golgi apparatus. Journal of Cell Science 107, 1321-1331.

Mocellin, S., Rossi, C.R., Pilati, P. \& Nitti, D. (2005). Tumor Necrosis Factor, Cancer and Anticancer Therapy. Cytokine \& Growth Factor Reviews 16, 35-53.

Morris, P.G. \& Fornier, M.N. (2008). Microtubule Active Agents: Beyond the Taxane Frontier. Clinical Cancer Research 14, 7167-7172.

Mukherjee, S., Gnosh, R.N. \& Maxfield, F.R. (1997). Endocytosis. Physiological Reviews 77, 759803.

Mukhopadhyay, S. \& Gordon, S. (2004). The Role of Scavenger Receptors in Pathogen Recognition and Innate Immunity. Immunobiology 209, 39-49.

Mullins, D.W., Burger, C.J. \& Elgert, K.D. (1999). Paclitaxel Enhances Macrophage IL-12 Production in Tumor-Bearing Hosts Through Nitric Oxide. The Journal of Immunology 162, 68116818. 
Munder, M., Mallo, M., Eichmann, K. \& Modolell, M. (1998). Murine Macrophages Secrete Interferon- $\gamma$ upon Combined Stimulation with Interleukin (IL)-12 and -18: A Novel Pathway of Autocrine Macrophage Activation. The Journal of Experimental Medicine 187, 2103-2108.

Murray, R.Z., Kay, J.G., Sangermani, D.G. \& Stow, J.L. (2005). A Role for the Phagosome in Cytokine Secretion. Science 310, 1492-1495.

Nagelkerke, J.F., Barto, K.P. \& van Berkel, T.J. (1983). In-vivo and in-vitro Uptake and Degradation of Acetylated Low Density Lipoprotein by Rat Liver Endothelial, Kupffer, and Parenchymal Cells. Journal of Biological Chemistry 258, 12221-12227.

Nakano, M., Tominaga, K., Saito, S., Kirikae, F., Lin, S., Fumero, C.L., Ojima, I. \& Kirikae, T. (1999). Lipopolysaccharide- and paclitaxel (Taxol)-induced tolerance in murine peritoneal macrophages. Journal of Endotoxin Research 5, 102-106.

Nogales, E. (2000). Structural Insights into Microtubule Function Annual Review of Biochemistry 69, 277-302.

Nogales, E., Whittaker, M., Milligan, R.A. \& Downing, K.H. (1999). High Resolution Model of the Microtubule. Cell 96, 79 - 88.

O'Riordan, M., Yi, C.H., Gonzales, R., Lee, K.-D. \& Portnoy, D.A. (2002). Innate Recognition of Bacteria by a Macrophage Cytosolic Surveillance Pathway. Proceedings of the National Academy of Sciences of the United States of America 99, 13861-13866.

Oda, H., Stockert, R.J., Collins, C., Wang, H., Novikoff, P.M., Satir, P. \& Wolkoff, A.W. (1995). Interaction of the Microtubule Cytoskeleton with Endocytic Vesicles and Cytoplasmic Dynein in Cultured Rat Hepatocytes. Journal of Biological Chemistry 270, 15242-15249.

Oliveira, C.A., Kashman, Y. \& Mantovani, B. (1996). Effects of Latrunculin A on Immunological Phagocytosis and Macrophage Spreading-Associated Changes in the F-actin/G-actin Content of the Cells. Chemico-Biological Interactions 100, 141-153.

Opal, S.M. \& DePalo, V.A. (2000). Anti-Inflammatory Cytokines. Chest 117, 1162-1172.

Orr, G.A., Verdier-Pinard, P., McDaid, H. \& Horwitz, S.B. (2003). Mechanisms of Taxol Resistance Related to Microtubules. Oncogene 22, 7280-7295.

Ostlund, R.E.J., Pfleger, B. \& Schonfeld, G. (1979). Role of Microtubules in Low Density Lipoprotein Processing by Cultured Cells. Journal of Clinical Investigations 63, 75-84.

Panchagnula, R. (1998). Pharmaceutical Aspects of Paclitaxel. International Journal of Pharmaceutics 172, 1-15. 
Parish, C.R. \& Warren, H.S. (2001). In-Vivo Assays for Lymphocyte Function : Use of the Intracellular Fluorescent Dye CFSE to Monitor Lymphocyte Migration and Proliferation. Current Protocols in Immunology Supplement 49, 4.9.1-4.9.10.

Peachman, K., K., Rao, M., Palmer, D., R., Zidanic, M., Wellington, S., Alving, C., R. \& Rothwell, S., W. (2004). Functional Microtubules are Required for Antigen Processing by Macrophages and Dendritic Cells. Immunology Letters 95, 13-24.

Peiser, L., Gough, P.J., Kodama, T. \& Gordon, S. (2000). Macrophage Class A Scavenger Receptor-Mediated Phagocytosis of Escherichia coli: Role of Cell Heterogeneity, Microbial Strain, and Culture Conditions In Vitro. Infection and Immunity 68, 1953-1963.

Peiser, L., Mukhopadhyay, S. \& Gordon, S. (2002). Scavenger Receptors in Innate Immunity. Current Opinion in Immunology 14, 123-128.

Perera, P., Qureshi, N. \& Vogel, S. (1996). Paclitaxel (Taxol)-induced NFKB translocation in Murine Macrophages. Infection and Immunity 64, 878-884.

Pesanti, E.L. \& Axline, S.G. (1975). Phagolysosome Formation in Normal and Colchicine-treated Macrophages. Journal of Experimental Medicine 142, 903-913.

Platanias, L.C. (2005). Mechanisms of type-I- and type-II Interferon-Mediated Signalling. Nature Reviews Immunology 5, 375-386.

Plüddemann, A., Neyen, C. \& Gordon, S. (2007). Macrophage Scavenger Receptors and HostDerived Ligands. Methods 43, 207-217.

Pozzolini, M., Scarfi, S., Benatti, U. \& Giovine, M. (2003). Interference in MTT Cell Viability Assay in Activated Macrophage Cell Line. Analytical Biochemistry 313, 338-341.

Prell, R.A., Gearin, L., Simmons, A., VanRoey, M. \& Jooss, K. (2006). The anti-tumor efficacy of a GM-CSF-secreting tumor cell vaccine is not inhibited by docetaxel administration. Cancer Immunology and Immunotherapy 55, 1285-1293.

Raetz, C.R.H. (1990). Biochemistry of Endotoxins. Annual Review Of Biochemistry 59, 129-170.

Ralph, P. \& Nakoinz, I. (1977a). Antibody-Dependent Killing of Erythrocyte and Tumor Targets by Macrophage-Related Cell Lines: Enhancement by PPD and LPS. The Journal of Immunology 119, 950-954.

Ralph, P. \& Nakoinz, I. (1977b). Direct Toxic Effects of Immunopotentiators on Monocytic, Myelomonocytic, and Histiocytic or Macrophage Tumor Cells in Culture. Cancer Research 37, 546550 . 
Rao, P., Falk, L., Dougherty, S., Sawada, T. \& Pluznik, D. (1997). Colchicine Down-Regulates Lipopolysaccharide-Induced Granulocyte-Macrophage Colony-Stimulating Factor Production in Murine Macrophages. The Journal of Immunology 159, 3531-3539.

Raschke, W.C., Baird, S., Ralph, P. \& Nakoinz, I. (1978). Functional Macrophage Cell Lines Transformed by Abelson Leukemia Virus. Cell 15, 261-267.

Reaven, E.P. \& Axline, S.G. (1973). Subplasmalemmal Microfilaments and Microtubules in Resting and Phagocytizing Cultivated Macrophages The Journal of Cell Biology 59, 12-27.

Rebres, R. \& Roach, T. (2004) Passage of RAW 264.7 cells for FXM Experiments In: AfCS Procedure Protocol PP00000226.

Renwick, L.C., Donaldson, K. \& Clouter, A. (2001). Impairment of Alveolar Macrophage Phagocytosis by Ultrafine Particles. Toxicology and Applied Pharmacology 172, 119-127.

Risinger, A.L., Giles, F.J. \& Mooberry, S.L. (2008). Microtubule Dynamics as a Target in Oncology. Cancer Treatment Reviews, 255 - 261.

Robinson, M.J. (2009) The Effect of Microtubule Stabilizing Drugs on Immune-Mediated Exocytosis. In: School of Biological Sciences. Victoria University of Wellington, Master of Science in Cell and Molecular Biology, p. 1-128.

Robinson, M.J., Ronchese, F., Miller, J.H. \& La Flamme, A.C. (2009). Paclitaxel Inhibits Killing by Murine Cytotoxic T Lymphocytes In-Vivo but not In-Vitro. Immunology and Cell Biology 88, 291296.

Rogers, S.L. \& Gelfand, V.I. (2000). Membrane Trafficking, Organelle Transport, and the Cytoskeleton. Current Opinion in Cell Biology 12, 57-62.

Rothe, H., Hartmann, B., Geerlings, P. \& Kolb, H. (1996). Interleukin-12 Gene-Expression of Macrophages Is Regulated by Nitric Oxide. Biochemical and Biophysical Research Communications 224, 159-163.

Rowinsky, E.K. \& Donehower, R.C. (1995). Paclitaxel (Taxol). The New England Journal of Medicine 332, 1004-1014.

Russell, D.G., VanderVen, B.C., Glennie, S., Mwandumba, H. \& Heyderman, R.S. (2009). The Macrophage Marches on its Phagosome: Dynamic Assays of Phagosome Function. Nature Reviews Immunology 9, 594-600.

Sahin, F., Celik, H.A., Aydin, H.H., Oktem, G., Omay, S.B. \& Saydam, G. (2008). The Interaction between Taxoids and Serine/Threonine Protein Phosphatase Activities During Taxane-Induced Apoptosis of HL-60 Leukemic Cells. Hematology 13, 215-223. 
Sakagami, H., Kishino, K., Amano, O., Kanda, Y., Kunii, S., Yokote, Y., Oizumi, H. \& Oizumi, T. (2009). Cell Death Induced by Nutritional Starvation in Mouse Macrophage-like RAW264.7 Cells. Anticancer Research 29, 343-347.

Santos, J.L., Andrade, A.A., Dias, A.A.M., Bonjardim, C.A., Reis, L.F.L., Teixeira, S.M.R. \& Horta, M.F. (2006). Differential sensitivity of C57BL/6 (M-1) and BALB/c (M-2) macrophages to the stimuli of IFN-gamma/LPS for the production of NO: Correlation with iNOS mRNA and protein expression. Journal of Interferon and Cytokine Research 26, 682-688.

Sashidhara, K.V., White, K.N. \& Crews, P. (2009). A Selective Account of Effective Paradigms and Significant Outcomes in the Discovery of Inspirational Marine Natural Products. Journal of Natural Products 72, 588-603.

Scheringa, M. \& Marquet, R. (1990). TNF: A Brief Review with Emphasis on its Antitumor Activity. Biotherapy 2, 275-281.

Schmidt, H.H.H.W. (1995). Determination of Nitric Oxide via Measurement of Nitrite and Nitrate in Culture Media. Biochemica 2, 22.

Schmidt, M. \& Bastians, H. (2007). Mitotic Drug Targets and the Development of Novel AntiMitotic Anticancer Drugs. Drug Resistance Updates 10, 162-181.

Schroder, K., Hertzog, P.J., Ravasi, T. \& Hume, D.A. (2004). Interferon- $\gamma$ : an Overview of Signals, Mechanisms and Functions. Journal of Leukocyte Biology 75, 163-189.

Schroder, K., Sweet, M.J. \& Hume, D.A. (2006). Signal Integration between IFN- $\gamma$ and TLR Signalling Pathways in Macrophages. Immunobiology 211, 511-524.

Schroer, T.A. \& Sheetz, M.P. (1991). Functions of Microtubule-Based Motors. Annual Review of Physiology 53, 629-652.

Schulze, E. \& Kirschner, M. (1987). Dynamic and Stable Populations of Microtubules in Cells. The Journal of Cell Biology 104, 277-288.

Shen, L.-H., Zhou, L., Wang, B.-Y., Pu, J., Hu, L.-H., Chai, D.-J., Wang, L., Zeng, J.-Z. \& He, B. (2008). Oxidized Low-Density Lipoprotein Induces Differentiation of RAW264.7 Murine Macrophage Cell Line into Dendritic-like Cells. Atherosclerosis 199, 257-264.

Shimazu, R., Akashi, S., Ogata, H., Nagai, Y., Fukudome, K., Miyake, K. \& Kimoto, M. (1999). MD-2, a molecule that confers lipopolysaccharide responsiveness on toll- like receptor 4. Journal of Experimental Medicine 189, 1777-1782.

Si, M.-S., Imagawa, D.K., Ji, P., Wei, X., Holm, B., Kwok, J., Lee, M., Reitz, B.A. \& Borie, D.C. (2003). Immunomodulatory Effects of Docetaxel on Human Lymphocytes. Investigational New Drugs 21, 281-290. 
Smythe, E. \& Warren, G. (1991). The Mechanism of Receptor-Mediated Endocytosis. European Journal of Biochemistry 202, 689-699.

Song, Y.W., Kim, H.-A., Baek, H.-J., Lee, E.-B., Chung, E.-S. \& Hong, K.-M. (1998). Paclitaxel Reduces Anti-dsDNA Antibody titer and BUN, Prolonging Survival in Murine Lupus. International Journal of Immunopharmacology 20, 669-677.

Sonnenschein, R.N., Johnson, T.A., Tenney, K., Valeriote, F.A. \& Crews, P. (2006). A Reassignment of (-)-Mycothiazole and the Isolation of a Related Diol. Journal of Natural Products 69, 145-147.

Sorger, P.K., Dobles, M., Tournebize, R. \& Hyman, A.A. (1997). Coupling Cell Division and Cell Death to Microtubule Dynamics. Current Opinion in Cell Biology 9, 807-814.

Sosroseno, W., Barid, I., Herminajeng, E. \& Susilowati, H. (2002). Nitric oxide production by a murine macrophage cell line (RAW264.7) stimulated with lipopolysaccharide from Actinobacillus actinomycetemcomitans. Oral Microbiology Immunology 17, 72-78.

Sparrow, C.P., Parthasarathy, S. \& Steinberg, D. (1989). A Macrophage Receptor That Recognizes Oxidised Low Density Lipoprotein but Not Acetylated Low Density Lipoprotein. The Journal of Biological Chemisty 264, 2599-2604.

Spector, I., Shochet, N.R., Blasberger, D. \& Kashman, Y. (1989). Latrunculins - Novel Marine Macrolides that Disrupt Microfilament Organization and Affect Cell Growth: I. Comparison with Cytochalasin D. Cell Motility and the Cytoskeleton 13, 127-144.

Spratlin, J. \& Sawyer, M.B. (2007). Pharmacogenetics of Paclitaxel Metabolism. Critical Reviews in Oncology/Hematology 61, 222-229.

Stephan, Z.F. \& Yurachek, E.C. (1993). Rapid Fluorometric Assay of LDL Receptor Activity by DiI-labeled LDL. Journal of Lipid Research 34, 325-330.

Stoltz, D.A., Nelson, S., Kolls, J.K., Zhang, P., Bohm Jr, R.P., Murphey-Corb, M. \& Bagby, G.J. (2000). In vitro ethanol suppresses alveolar macrophage TNF-alpha during Simian immunodeficiency virus infection. American Journal of Respiratory and Critical Care Medicine $161,135-140$.

Stuehr, D. \& Nathan, C. (1989). Nitric oxide. A Macrophage Product Responsible for Cytostasis and Respiratory Inhibition in Tumor Target Cells. Journal of Experimental Medicine 169, 15431555 .

Stuehr, D.J. \& Marletta, M.A. (1985). Mammalian nitrate biosynthesis: Mouse macrophages produce nitrite and nitrate in response to Escherichia coli lipopolysaccharide. Proceedings of the National Academy of Sciences of the United States of America 82, 7738-7742.

Stuehr, D.J. \& Marletta, M.A. (1987). Induction of nitrite/nitrate synthesis in murine macrophages by BCG infection, lymphokines, or interferon-gamma. J Immunol 139, 518-525. 
Su, S.-H., Chen, H.-I. \& Jen, C. (2001). C57BL/6 and BALB/c Bronchoalveolar Macrophages Respond Differently to Exercise. The Journal of Immunology 167, 5084-5091.

Subramanian, S., Roberts, C.L., Hart, A., Martin, H.M., Edwards, S.W., Rhodes, J.M. \& Campbell, B.J. (2008). Replication of Colonic Crohn's Disease Mucosal Escherichia Coli Isolates within Macrophages and their Susceptibility to Antibiotics. Antimicrobial Agents and Chemotherapy 52, 427-434.

Sukumaran, S.K., Shimada, H. \& Prasadarao, N.V. (2003). Entry and Intracellular Replication of Escherichia coli K1 in Macrophages Require Expression of Outer Membrane Protein A. Infection and Immunity 71, 5951-5961.

Sun, J., Zhang, X., Broderick, M. \& Fein, H. (2003). Measurement of Nitric Oxide Production in Biological Systems by using Griess Reaction Assay. Sensors 3, 276-284.

Suzuki, E. \& Umezawa, K. (2006). Inhibition of Macrophage Activation and Phagocytosis by a Novel NF-KB inhibitor, dehydroxymethylepoxyquinomicin. Biomedicine and Pharmacotherapy 60, 578-586.

Takeda, K., Kaisho, T. \& Akira, S. (2003). Toll--Like Receptors. Annual Review of Immunology 21, 335-376.

Tanaka, J. \& Higa, T. (1996). Zampanolide, a New Cytotoxic Macrolide from a Marine Sponge. Tetrahedron Letters 37, 5535-5538.

Taylor, P.R., Martinez-Pomares, L., Stacey, M., Lin, H.H., Brown, G.D. \& Gordon, S. (2005). Macrophage Receptors and Immune Recognition Annual Review of Immunology 23, 901-944.

Toyohara, A. \& Inaba, K. (1989). Transport of Phagosomes in Mouse Peritoneal Macrophages. Journal of Cell Science 94, 143-153.

Tracey, K.J. \& Cerami, A. (1993). Tumor Necrosis Factor, Other Cytokines and Disease. Annual Review of Cell Biology 9, 317-343.

Tracey, M.D., Kevin J. \& Cerami, P.D., Anthony. (1994). Tumor Necrosis Factor: A Pleiotropic Cytokine and Therapuetic Target. Annual Review of Medicine 45, 491-503.

Trinchieri, G. (2003). Interleukin-12 and the Regulation of Innate Resistance and Adaptive Immunity. Nature Reviews Immunology 3, 133-146.

Tsavaris, N., Kosmas, C., Vadiaka, M., Kanelopoulos, P. \& Boulamatsis, D. (2002). Immune Changes in Patients with Advanced Breast Cancer undergoing Chemotherapy with Taxanes. British Journal of Cancer 87, 21.

Underhill, D.M. \& Ozinsky, A. (2002). Phagocytosis Of Microbes: Complexity in Action. Annual Review of Immunology 20, 825-852. 
van Horssen, R., ten Hagen, T.L.M. \& Eggermont, A.M.M. (2006). TNF- $\alpha$ in Cancer Treatment: Molecular Insights, Antitumor Effects, and Clinical Utility. Oncologist 11, 397-408.

Vandenabeele, P., Declercq, W., Beyaert, R. \& Fiers, W. (1995). Two Tumour Necrosis Factor Receptors: Structure and Function. Trends in Cell Biology 5, 392-399.

Vassalli, P. (1992). The Pathophysiology of Tumor Necrosis Factors. Annual Review of Immunology 10, 411-452.

Vaudaux, P. \& Waldvogel, F.A. (1979). Gentamicin Antibacterial Activity in the Presence of Human Polymorphonuclear Leukocytes. Antimicrobial Agents and Chemotherapy 16, 743-749.

von Figura, K., Kresse, H., Meinhard, U. \& Holtfrerich, D. (1978). Studies on Secretion and Endocytosis of Macromolecules by Cultivated Skin Fibroblasts. Effects of Anti-Microtubular Agents on Secretion and Endocytosis of Lysosomal Hydrolases and of Sulphated Glycosaminoglycans. Biochemical Journal 170, 313-320.

Walter, R.J., Berlin, R.D., Pfeiffer, J.R. \& Oliver, J.M. (1980). Polarization of Endocytosis and Receptor Topography on Cultured Macrophages. The Journal of Cell Biology 86, 199-211.

Wan, C.P., Park, C.S. \& Lau, B.H. (1993). A Rapid and Simple Microflurometric Phagocytosis Assay. Journal of Immunological Methods 162, 1-7.

Wani, M.C., Taylor, H.L., Wall, M.E., Coggon, P. \& McPhail, A.T. (1971). Plant antitumor agents. VI. Isolation and Structure of Taxol, a Novel Antileukemic and Antitumor Agent from Taxus brevifolia. Journal of the American Chemical Society 93, 2325-2327.

West, L.M., Northcote, P.T. \& Battershill, C.N. (2000). Peloruside A: A Potent Cytotoxic Macrolide Isolated from the New Zealand Marine Sponge Mycale sp. The Journal of Organic Chemistry 65, 445-449.

Wilmes, A., Bargh, K., Kelly, C., Northcote, P.T. \& Miller, J.H. (2007). Peloruside A Synergizes with Other Microtubule Stabilizing Agents in Cultured Cancer Cell Lines. Molecular Pharmaceutics 4, 269-280.

Xie, J., Kolls, J., Bagby, G. \& Greenberg, S.S. (1995). Independent suppression of nitric oxide and TNF-alpha in the lung of conscious rats by ethanol. FASEB Journal 9, 253-261.

Yao, W., Li, K. \& Liao, K. (2009). Macropinocytosis Contributes to the Macrophage Foam Cell Formation in RAW264.7 Cells. Acta biochimica et biophysica Sinica 41, 773-780.

Yates, R.M., Hermetter, A., Taylor, G.A. \& Russell, D.G. (2007). Macrophage Activation Downregulates the Degradative Capacity of the Phagosome. Traffic 8, 241-250.

Young, H. \& Hardy, K. (1995). Role of interferon- $\gamma$ in Immune Cell Regulation. Journal of Leukocyte Biology 58, 373-381. 
Zhang, X. \& Mosser, D. (2008). Macrophage Activation by Endogenous Danger Signals. The Journal of Pathology 214, 161-178.

Zhou, J. \& Giannakakou, P. (2005). Targeting Microtubules for Cancer Chemotherapy. Current Medicinal Chemistry - Anti-Cancer Agents 5, 65-71.

Zimmer, S.M., Liu, J., Clayton, J.L., Stephens, D.S. \& Snyder, J.P. (2008). Paclitaxel Binding to Human and Murine MD-2. Journal of Biological Chemistry 283, 27916-27926.

Zitvogel, L., Apetoh, L., Ghiringhelli, F., Andre, F., Tesniere, A. \& Kroemer, G. (2008). The Anticancer Immune Response: Indispensable for therapeutic success? The Journal of Clinical Investigation 118, 1991-2001. 


\section{Appendices}

\section{Appendix A: Recipes}

\section{Phosphate Buffered Saline (PBS) (2 Litres)}

$\mathrm{Na}_{2} \mathrm{HPO}_{4} \quad 8.7 \mathrm{mM}$

$\mathrm{NaH}_{2} \mathrm{PO}_{4} \quad 1.3 \mathrm{mM}$

$\mathrm{NaCl} \quad 145 \mathrm{mM}$

2 litres sterile double distilled water

\section{FACS Buffer:}

$2 \%$ FCS

0.1\% Sodium Azide

97.9\% PBS

\section{Complete $\mathbf{T}$ cell media (CTCM) - media for cell line culture}

85.9\% Dulbecco's Modified Eagle Medium (DMEM, Invitrogen)

1\% Non-essential Amino Acids (10mM; 100x, Invitrogen)

1\% Penicillin/Streptomycin (Penstrep; 100000 U/mL, Invitrogen)

1\% L-Glutamine (200mM, Invitrogen)

1\% HEPES (1M, Sigma)

$0.1 \% \beta$-Mercaptoethanol (1000 x 55mM, Invitrogen)

$10 \%$ sterile-filtered heat-inactivated FCS 


\section{$\underline{\text { Wash Buffer }}$}

96\% Dulbecco's Modified Eagle Medium (DMEM, Invitrogen)

$3 \%$ HEPES (1M, Sigma)

1\% Penicillin/Streptomycin (Penstrep; $100000 \mathrm{U} / \mathrm{mL}$, Invitrogen)

$\underline{\text { Experimental Media - for phagocytosis assays }}$

95\% DMEM

$5 \%$ sterile filtered heat-inactivated FCS

\section{Freezing Media - for Liquid Nitrogen cell storage}

90\% sterile filtered heat-inactivated FCS

10\% DMSO (Sigma)

\section{$\underline{\text { MTT solution }}$}

$5 \mathrm{mg} \cdot \mathrm{mL}^{-1}$ MTT in $1 \mathrm{x}$ dPBS, sterile filtered

Kept Sterile, MTT - toxic and light sensitive, handled using protective clothing, and stored for 4 weeks or longer at $4^{\circ} \mathrm{C}$, protected from light. Discarded appropriately.

\section{$\underline{\text { MTT Solubiliser }}$}

$10 \% \mathrm{w} / \mathrm{v}$ SDS in $0.01 \mathrm{~N} \mathrm{HCl}$

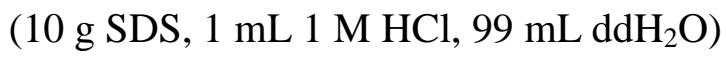




\section{Greiss Reaction reagents}

Solution A: $1 \%$ w.v sulphanilamide in $2.5 \%$ phosphoric acid

Solution B: $0.1 \%$ w.v N-(1-naphthyl) ethylenediamine in $2.5 \%$ phosphoric acid

\section{Luria- Bertani Broth (LB broth) $(500 \mathrm{ml})$}

$5 \mathrm{~g} \mathrm{NaCl}$

5 g Tryptone

$2.5 \mathrm{~g}$ Yeast extract

$500 \mathrm{ml}$ sterile $\mathrm{ddH}_{2} \mathrm{O}$, autoclaved and cooled to touch

$34 \mu \mathrm{g} / \mathrm{ml}$ chloramphenicol added and flame sealed to store at room temperature.

\section{Luria-Bertani Agar (LB agar) $(500 \mathrm{ml})$}

$5 \mathrm{~g} \mathrm{NaCl}$

5 g Tryptone

5 g BactoAgar

$2.5 \mathrm{~g}$ Yeast extract

$500 \mathrm{ml}$ sterile $\mathrm{ddH}_{2} \mathrm{O}$, autoclaved and cooled to touch.

$34 \mu \mathrm{g} / \mathrm{ml}$ chloramphenicol added, agar poured into $20 \mathrm{~mm}$ petri dishes, allowed to solidify and plates stored upside down at $4{ }^{\circ} \mathrm{C}$.

\section{$\underline{\text { Krebs Buffer }}$}

$130 \mathrm{mM} \mathrm{NaCl}$

$1.3 \mathrm{mM} \mathrm{KCl}$

$2.2 \mathrm{mM} \mathrm{MgSO}_{4} \cdot 6 \mathrm{H}_{2} \mathrm{O}$ 


\section{$1.2 \mathrm{mM} \mathrm{KH}_{2} \mathrm{PO}_{4}$}

\section{0 mM HEPES}

$10 \mathrm{mM}$ D-glucose

$500 \mathrm{ml}$ sterile double distilled water, $\mathrm{pH} 7.4$

\section{Staining protocol for cytospins}

Fixation: 10 dips in methanol

Allow excess methanol to drip off

Staining: 10 dips in $100 \%$ Geimsa

Allow excess Geimsa to drip off

Wash: $10-20$ dips in sterile distilled water, followed by rinsing gently in sterile distilled water to remove excess Geimsa stain.

Air dry with sample facing upwards. 


\section{Appendix B: Antibody List}

$\begin{array}{ll}\text { CD11b-bio } & \text { Serotec } \\ \text { F4/80-Fitc } & \text { Serotec } \\ \text { Streptavidin-Cyc } & \text { BD Bioscience } \\ \text { IgG2- } \alpha \text {-Fitc } & \text { BD Bioscience } \\ \text { IgG2- } \alpha \text {-bio } & \text { BD Bioscience } \\ \text { Anti-mouse-CD16/32 (24G2) (FcR block) } & \text { BD Bioscience }\end{array}$

